\title{
The Analysis of Geomagnetic Storm-Time Variations
}

\author{
Nigel Charles Davidson
}

A thesis submitted in fulfilment of the requirements

for the degree of Doctor of Philosophy

$$
\text { to the }
$$

University of Edinburgh

1992

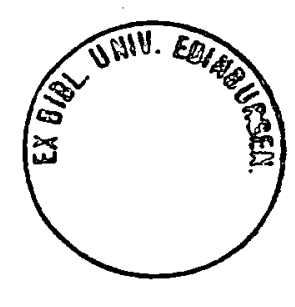


My son, there is something else to watch out for. There is no end to the writing of books, and too much study will wear you out.

After all this, there is only one thing to say: Fear God, and obey his commands, because this is all that man was created for. God is going to judge everything we do, whether good or bad, even things done in secret.

Ecclesiastes: Chapter 12 verses 12 to 14. Good News Bible. 


\section{Abstract}

The Earth is bathed in an ever changing magnetic environment due to fluctuations in the solar wind. The external field induces currents within the Earth which cause a secondary internal field. The ratio of internal to external parts of the magnetic potential is known as the response and may be derived from measurements of the field at the surface. The response of the Earth is dependent on the spatial form of the field and distribution of conductivity within the Earth. The analysis of numerous geomagnetic storms, large disturbances in the field surrounding the whole Earth, is presented with the aim of determining a reliable response function which enables an estimate to be made of the average conductivity of the upper mantle.

The compilation of a database of geomagnetic storms was a major part of the work. All the suitable storm events were selected between 1957 and 1982 to give 44 storms. The entire set of hourly values were checked for errors and corrections made where necessary. Where data were missing their values were interpolated using information from nearby observatories.

The lower the frequency of external magnetic variations the deeper the penetration into the Earth. The frequency content of geomagnetic storms allows depths approaching $1500 \mathrm{~km}$ to be investigated. The observations of magnetic field were Fourier transformed and attention focussed on the Fourier coefficients of the lowest frequencies, 0.03 to 1 cycle per day.

From Spherical Harmonic Analysis in the frequency domain it was found that a pure $P_{1}^{0}$ spherical harmonic model is acceptable for the spatial form of the field at the frequencies of most interest. Thus the source is assumed to be a simple ring current in common with most of the previous research. The Fourier coefficients of the $X$ and $Z$ magnetic components were then fitted to the appropriate $P_{1}^{0}$ model which allows the separate internal and external parts to be evaluated. A robust method, to reduce the influence of anomalous values, was used for determining the 
optimum fit to the Fourier coeffcients. The technique was assessed by examining the distribution of residuals.

An estimate of the response was then found for each of the storms. An average was calculated, again using the robust technique, and error estimates determined also in a manner resistant to outliers. The resultant response was input to various inversion routines and forward modelling carried out. It was found that models with a steep rise in conductivity between 600 and $700 \mathrm{~km}$ depth, with a contrast of approximately two orders of magnitude, gave the best fit to the determined response function. 


\section{Acknowledgements}

Firstly I would like to thank my main supervisor Bruce Hobbs for his help during the course of the project. I am also grateful for the advice given by Roger Banks my second supervisor. Discussions with Phil Jones, Ronnie Parr and Dean Livelybrooks were an added help.

In the initial stages facilities at the British Geological Survey, Murchinson House, Edinburgh were used for accessing the compact disc and I would like to thank David Barraclough and David Kerridge for their assistance in this matter. The EUCS User Support team followed up the many queries I had regarding the computer system and deserve mention.

The financial support was provided by the Natural Environment Research Council and additional awards from IAGA funds were received for conference attendance.

I also would like to add a word of thanks to the many observatory staff worldwide who have and continue to log the magnetic field. Without their work such a study would not be possible.

Finally I wish to thank my parents who have continually supported me throughout my years as a student. During my time in Edinburgh I have been greatly helped by friends made at Duncan St. Baptist Church and also by certain Christian brothers from lands very distant from Scotland. To these people who are one in Christ I dedicate this thesis. 


\section{Contents}

$\begin{array}{ll}\text { Abstract } & 3\end{array}$

Acknowledgements $\quad 5$

1 Introduction $\quad 9$

1.1 Preamble .................... 9

1.2 The Mantle ............................ 11

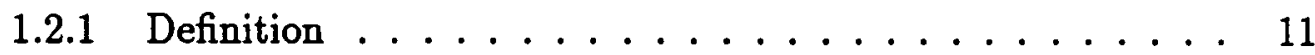

1.2 .2 Geophysical Understanding ............. 11

1.2.3 Conduction Mechanisms and Geological Interpretation . . 15

2 Magnetic Storms $\quad 17$

2.1 Introduction . . . . . . . . . . . . . . . . 17

2.2 The Earth's Magnetic Environment . . . . . . . . . . . 21

2.3 Storm Generation Theory . . . . . . . . . . . . . . 22

2.4 Application to Geomagnetic Deep Sounding . . . . . . . . . 23

2.4.1 General ................... . . 23

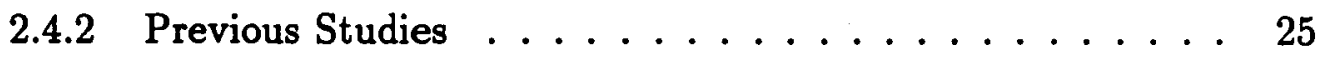

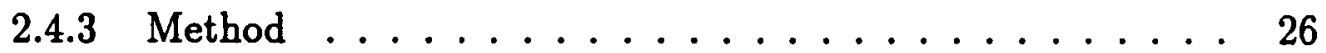

3 Theoretical Background $\quad 28$

3.1 Electromagnetic Theory ................. 28

3.1.1 The Basic Equations ............... . . 28

3.1.2 Electromagnetic Fields in an Ohmic Medium . . . . . . . 29

3.1.3 Electromagnetic Fields in and around the Earth. . . . . . . 31

3.2 Fourier Transformation . . . . . . . . . . . . . . 33 
3.2.1 The Discrete Fourier Transform . . . . . . . . . . . 33

3.2 .2 Window Functions . . . . . . . . . . . . 34

3.3 Spherical Harmonic Analysis . . . . . . . . . . . . . 37

3.3 .1 Introduction . . . . . . . . . . . . 37

3.3.2 SHA using Least Squares . . . . . . . . . . . . . 38

3.3.3 Separation of Internal and External Parts . . . . . . 40

4 Data Management 42

4.1 Compilation of Storm Database . . . . . . . . . . . . 42

4.1 .1 Data Access . . . . . . . . . . . . . . 42

4.1 .2 Data Retrieval . . . . . . . . . . . . 43

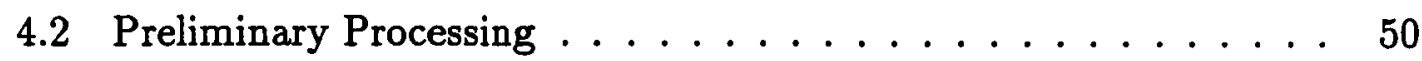

4.2 .1 Data Scrutiny . . . . . . . . . . . . . . 50

4.2.2 Conversion of Magnetograms to the Geomagnetic Dipole Frame of Reference . . . . . . . . . . . . 51

4.2.3 Removal of the Daily Variation . . . . . . . . . . 53

4.2 .4 Interpolation for Missing Data . . . . . . . . . . 61

5 Storm Analysis - FT 66

5.1 Background ...................... 66

5.1 .1 The Approach of Analysis . . . . . . . . . 66

5.1.2 Preprocessing the Time Series . . . . . . . . . . 66

5.2 Calculating the Fourier Coefficients . . . . . . . . . . 67

5.3 Application of the Window Function . . . . . . . . . . 69

5.4 The Shift of Storm Commencement . . . . . . . . . . . 70

5.5 Multiple Storm Events . . . . . . . . . . . . . . 74

6 Storm Analysis - SHA $\quad 80$

6.1 The Problem .................... . . 80

6.2 Previous Work . . . . . . . . . . . . . . 81

6.3 The Appropriate Spherical Harmonic Model . . . . . . . . . 82

6.4 Robust Estimation of the $P_{1}^{0}$ term . . . . . . . . . . . 87

6.4 .1 Introduction . . . . . . . . . . . 87

6.4.2 Robust Methodology ... . . . . . . . . . 88

6.4.3 Robust Determination of the $P_{1}^{0}$ Coefficient . . . . . . . 91

6.4 .4 Assessment . . . . . . . . . . . . . . 93

6.5 Separation of Internal and External Parts $\ldots \ldots \ldots \ldots$ 
7 Mid-Mantle Global Conductivity 101

7.1 Estimation of Global $P_{1}^{0}$ Response Function . . . . . . . . . 101

7.1.1 Calculation of the Average Response . . . . . . . . 101

7.1.2 Comparison with Previous Results . . . . . . . . . 111

7.2 A Global Conductivity Model . . . . . . . . . . . . . 111

7.2 .1 Introduction . . . . . . . . . . . . . 111

7.2.2 Inversion - Existence of a Solution and Model Determination113

7.2 .3 Forward Modelling . . . . . . . . . . . . . . 121

7.2 .4 Inversion - Inference . . . . . . . . . . . . 122

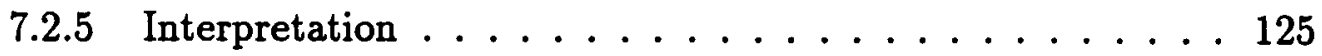

8 Conclusions 143

$\begin{array}{ll}\text { References } & 147\end{array}$

$\begin{array}{ll}\text { A Additional Figures and Tables } & 156\end{array}$ 


\section{Chapter 1}

\section{Introduction}

\subsection{Preamble}

In 1722 a London clockmaker, George Graham, found that the instrument he had made to measure the magnetic declination would sometimes show a change of up to $30^{\prime}$ in a few hours (Graham, 1724). Over two hundred and fifty years on, in March 1989, a time-varying magnetic field caused the collapse of the power system serving the Province of Quebec, Canada, cutting the electricity supply to six million people (Boteler, 1991). The contrast between these two events is dramatic though both are attributable to large rapid deviations in the magnetic field external to the Earth, i.e. to some sort of geomagnetic storm.

The effects of geomagnetic storms continue down into the Earth's interior to generate electric currents which in turn give rise to secondary magnetic fields. This is the process of electromagnetic induction (EM induction) which enables inference to the electrical conductivity of the Earth. Within a given medium time-varying magnetic fields of different frequencies induce currents at different depths. The frequency content of magnetic storms, Figure 2.2, therefore produces currents at a particular depth range within the Earth. The lower the frequency the greater the depth of penetration, the different sources of external field variations may be used to investigate conductivity from the surface to beyond $1000 \mathrm{~km}$ depth, see Figure 1.1 .

Various past studies have used magnetic storms to estimate the conductivity structure of the Earth, in most recent cases an individual storm was analysed. A study of six magnetic storms in the time domain (Marshall, 1980) and re-examination in the frequency domain (Hobbs, 1987) indicate that it is worthwhile to integrate the results from numerous storms. This multi-storm approach forms the basis for the work presented here. The improved availability of geomagnetic data, due to better storage 
Figure 1.1. Internal Structure of the Earth and Penetration Depths for different Geomagnetic Variations.

Seismic Structure

Region of Interest

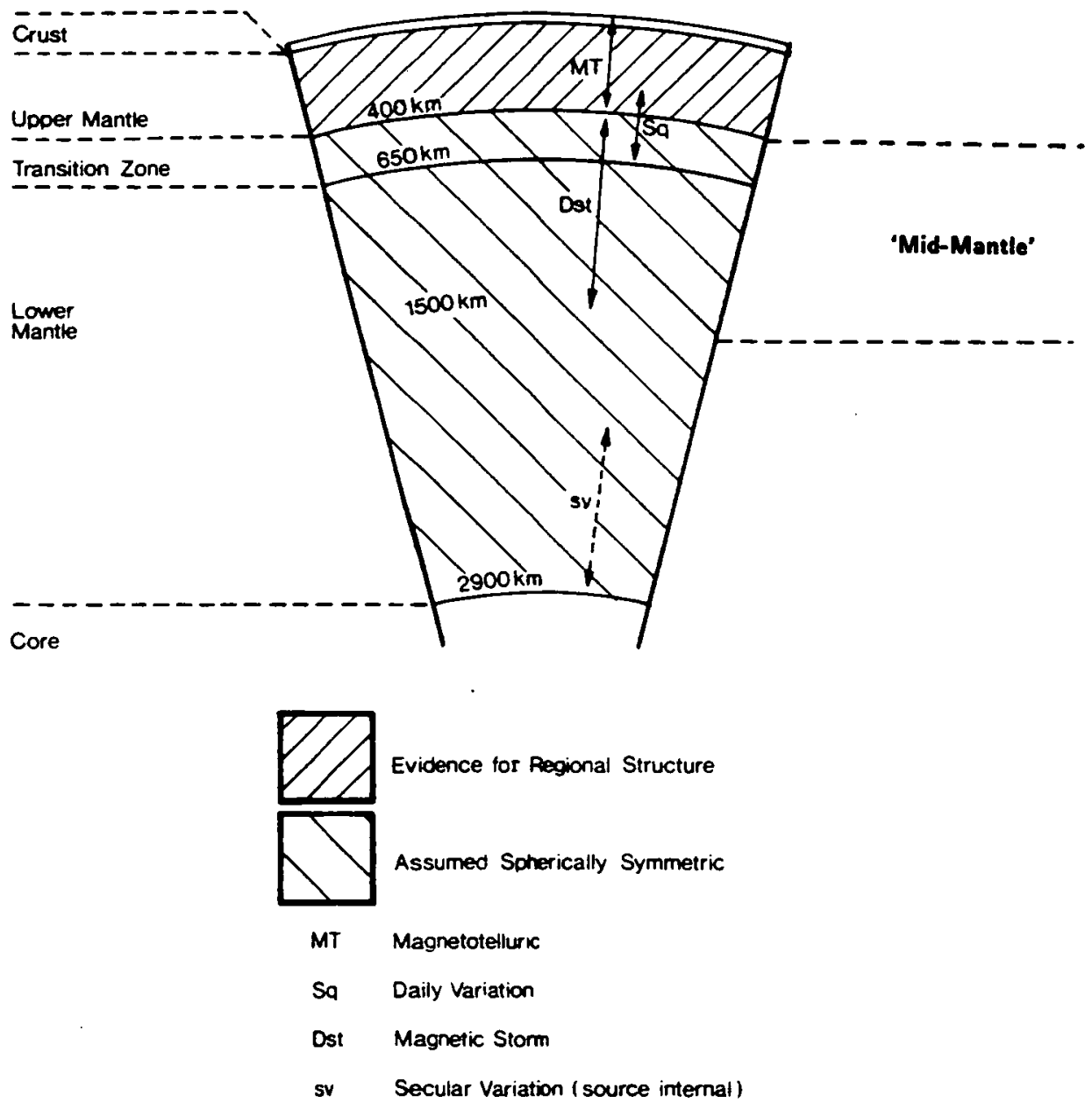


media i.e. compact disc, allowed forty four storms to be chosen. The objective was to determine the electromagnetic response of the Earth to a high level of confidence and so produce an optimum model of the Earth's average conductvity from around 400 to $1000 \mathrm{~km}$ depth.

In the derivation of the Earth's conductivity structure the value of determining the electromagnetic response of the Earth as accurately as possible was demonstrated by Parker (1970). To attempt to achieve this a large amount of data should be analysed in a considerate manner as Banks (1972) stated. Using various magnetic storms is almost analogous to repeating a laboratory experiment to reduce the uncertainty in the results, as the signal to noise ratio is improved by a factor of $\sqrt{N}$ for $N$ repetitions. Therefore, by treating many storms with an analysis strategy designed for their specfic nature it is desired to similarly refine the Earth's electromagnetic response and thus improve estimation of the global conductivity profile. To date a number of different models have been proposed for the conductivity of the mid-mantle, these are discussed in the following section.

\subsection{The Mantle}

\subsubsection{Definition}

The internal structure of the Earth has been classified into three main divisions, the core, mantle and crust. Evidence for these three regions is primarily based on seismic body waves radiating from earthquakes but is supported by the Earth's moment of inertia, free oscillation periods, theories of element segregation and electrical conductivity studies. From such investigations the mantle is defined to extend between depths of approximately $35 \mathrm{~km}$, shallower for oceans and deeper for continents, to $2900 \mathrm{~km}$. In this thesis the term mid-mantle is used to refer to a depth range of approximately 400 to $1500 \mathrm{~km}$. This is because only below $400 \mathrm{~km}$ can the Earth be assumed to be spherically symmetric and $1500 \mathrm{~km}$ is at the limit of penetration for external field variations. Figure 1.1 clarifies this point.

\subsubsection{Geophysical Understanding}

\section{Seismic}

Studies of seismic body waves, compressional and shear, within the mantle reveal an overall increase in velocity with depth. However, between approximately 60 and 250 $\mathrm{km}$ a zone of anomalously low velocity is evident. This has been refered to as the low 
Figure 1.2. Average $P$ wave Velocity for the Upper Half of the Mantle.

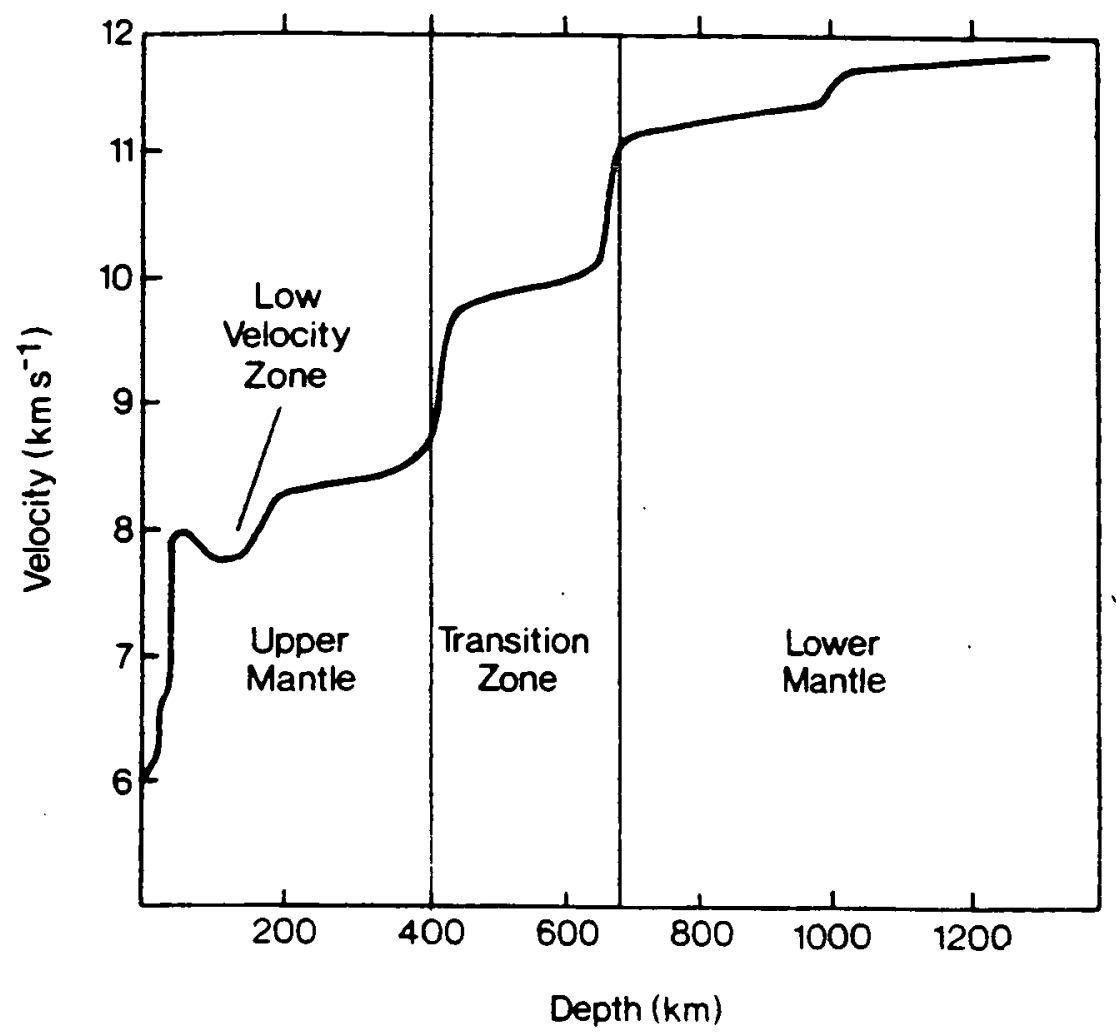

velocity zone and is most probably due to partial melt. This has been associated with the idea of a deformable layer, the asthenosphere, important in the theory of plate tectonics. At a depth of $400 \mathrm{~km}$ there is an abrupt increase in the seismic velocity and at $650 \mathrm{~km}$ (sometimes quoted as $670 \mathrm{~km}$ ) another rapid increase occurs. Between the depths of 400 and $650 \mathrm{~km}$ the positive velocity gradient is higher than elsewhere in the mantle, this region is called the transition zone. The average velocity profile for $P$ waves in the upper part of the mantle is shown in Figure 1.2.

To complement the results of body wave investigations surface waves may also be used to determine the structure of the mantle and are particulary useful for estimating lateral variations in velocity. From the current network of seismic stations features in the order of $2000 \mathrm{~km}$ may be resolved down to approximately $500 \mathrm{~km}$. Measurements of the group and phase velocity over different arcs allow an image of the mantle to be derived by the inverse procedure of tomography. The results, described in Anderson (1989), show that the velocity of the mantle underlying tectonic and young ocean regions is slower than average while continental shields are associated with higher seismic 
velocity. The deviations relative to the average velocity distribution are of the order of five percent and decrease with depth to less than two percent below $400 \mathrm{~km}$. Correlation with the Earth's surface tectonic features diminishes on the analysis of the longer period waves penetrating into the transition zone. Body waves have also been studied to reveal the same pattern of lateral heterogeneity of relatively slow velocity for tectonic regions to higher than average velocity for the mantle beneath shields. Very small lateral variations of one percent have been suggested for the lower mantle, below the transition zone, using body wave tomography. However, this method is not without difficulties and therefore the significance of this result should be treated with caution. The overall velocity depth profile deduced from body waves is supported by the study of surface waves.

\section{Electromagnetic}

The only other means of probing structure within the mid-mantle is by electromagnetic induction studies, thus EM induction is important as an additional source of evidence for physical changes. By measuring the electric and magnetic fields fluctuating with periods up to 3 hours, as done in the magnetotelluric method (MT) estimates for the conductivity of the uppermost few hundred kilometers may be made. The conductivity at greater depths can be derived from measurements of the Earth's response to time variant magnetic fields of longer periods. Field variations originating outside the Earth of up to a year period may be used to estimate conductivity down to $1500 \mathrm{~km}$. The deduction of conductivity below this depth is more problematic. In this instance the task is to determine the conductivity of the mantle that would filter the slowly varying field produced within the core, the secular variation (sv). For external fields there is knowledge as to the form of the primary field, however, the nature of the field variation originating internally is unknown.

The models that have been derived for the conductivity profile below $400 \mathrm{~km}$ show a general monotonic increase in conductivity down to the core-mantle boundary (CMB). The only exceptions to the increasing trend are at depths above $200 \mathrm{~km}$ where regional zones of anomalously high conductivity are indicated from MT, refer to Parkinson and Hutton $(1989 ; \mathrm{p} 287)$ for a review. There is some debate as to the nature of the rise in conductivity down to the CMB. Work by Lahiri and Price (1939) resulted in two limiting models, one of a smooth increase in conductivity down to $600 \mathrm{~km}$ and the other of a discontinuity at about the same depth. The analysis and modelling of Banks $(1969,1972)$ produced a model with a steep positive gradient around $400 \mathrm{~km}$ which was made deeper to between 500 and $600 \mathrm{~km}$ in the latter paper. The discontinuous 
Figure 1.3. The bounds on the Average Conductivity for the Mid-Mantle.

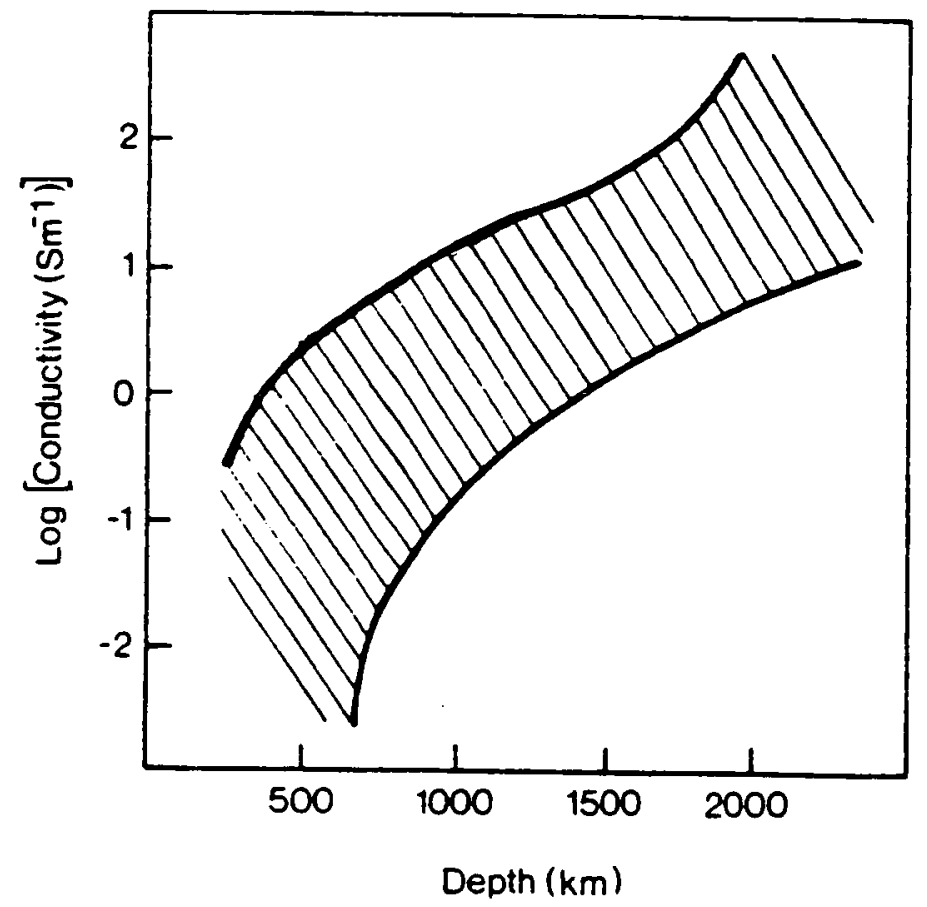

model of Lahiri and Price gave a change in conductivity from $10^{-3}$ to $1 \mathrm{Sm}^{-1}$, the second model of Banks reached a level of $2 \mathrm{Sm}^{-1}$ beyond which the rise was more gradual. However, a model proposed by Achache et al. (1981) presents a smoother increasing function. The conductivity depth models put forward by the forementioned researchers fit within the shaded region of Figure 1.3. This shaded area has been drawn to also include the majority of estimated conductivity depth profiles derived by other workers such as Rikitake (1966), Parker (1970), Jady (1975), Berdichevski et al. (1976) and Rokityansky (1982). Further models have been found for individual observatories which may lie outwith the marked region, however, it is the global average conductivity that is considered here. Therefore a range of views exists as to how the conductivity increases with depth. The question of lateral variability in conductivity may contribute to the suite of models and certainly above $400 \mathrm{~km}$ the conductivity is different for different locations (Roberts, 1986). However, there are a number of other reasons why different conductivity models may have been found including the type of analysis scheme, number and distribution of observatories, assumption of source field morphology and mathematical modelling techniques applied.

There are also various models put forward for the electrical state of the lower 
mantle, though this is almost inevitable considering the assumptions that must be made concerning the field produced by the core.

\subsubsection{Conduction Mechanisms and Geological Interpretation}

Within the crust and topmost mantle electrolytic conduction in fluids is the most important conduction mechanism. Most rocks at normal temperatures are nearly insulators and therefore conduction depends more on any fluid taking up fractures and pores than the actual composition of the rock. The water present at such depths contains dissolved salts so acts as a relatively good conductor. As well as the porosity and amount of water the configuration of the fractures is a significant factor on the conductivity. Brine is important as it is globally widespread though electrolytic conduction in fluids yields a conductivity less than metallic conductors and semiconductors. Metallic conductors such as graphite exist within the Earth and semiconductors are also present in the form of oxide and sulphide minerals. Some researchers conclude that graphite plays an important role in the conductivity of the crust and upper mantle, for example to explain high conductivity within the Canadian lower crust (Mareschal, 1992).

At around 60 to $250 \mathrm{~km}$ depth there is a decrease in seismic velocity which coincides with a zone of high conductivity included in some models of global conductivity (Larsen, 1977; Rokityansky, 1982; p155). This region is called the low velocity zone (Figure 1.2) and is probably due to partial melt. The increased temperatures at these depths melt the mantle material and so ions are more mobile and the conductivity higher. At greater depths the increased pressure prohibits melting and the mantle is solid.

The bulk composition of the mantle is believed to be peridotite which is a group name given to basic rocks with olivine as the major component. Evidence for a peridotite mantle is provided by basalts, inclusions in kimberlites, inclusions in diamonds, xenoliths and chondrite meteorites; a good introduction to the composition of the mantle is given within the compilation of papers by Decker and Decker (1982).

Peridotite is an ionic solid semiconductor with the formula $\left(\mathrm{Fe}_{0.1}, \mathrm{Mg}_{0.9}\right)_{2} \mathrm{SiO}_{4}$. At low temperatures the electrons are bound to the ions and peridotite is an insulator. However, with an increase in temperature there are two mechanisms that allow the conduction of electricity; ionic conduction may occur with the diffusion of ions through the crystal lattice, and charge transfer may also take place between neighbouring ions of different valence (eg. $\mathrm{Fe}^{3+}$ and $\mathrm{Fe}^{2+}$ ), this is called hopping conduction. It is generally agreed that hopping conduction is the dominant conduction process within the mantle (Heinz, 1991).

Seismic discontinuities are observed at depths of 400 and $650 \mathrm{~km}$ and the inferred 
density and pressure indicate that if the composition is assumed constant phase changes account for these steps in velocity. At the estimated temperature and pressure at 400 $\mathrm{km}$ depth, laboratory experiments show peroditic olivine undergoes a transition to the more compact spinel structure; in this form the hopping conduction mechanism is enhanced. The increased temperature and pressure at $650 \mathrm{~km}$ depth induces a transformation from spinel to perovskite and magnesiowustite. There are conflicting results on the conductivity of perovskite as Wood and Nell (1991) derived conductivity consistent with the results of GDS studies while Li and Jeanloz (1991a) found perovskite to have a lower conductivity and suggest the lower mantle is more iron rich or contains significant volatiles compared to the upper mantle and transition zone ( $\mathrm{Li}$ and Jeanloz, 1991b). The suggestion of a compositional change in addition to a structural phase transition, as proposed by $\mathrm{Li}$ and Jeanloz (1991a), has great implications for the dynamic state of the mantle as then there is a barrier to the transport of heat and mass which would rule out whole-mantle convection.

The electrical conductivity may therefore be used as a discriminator of candidate mantle mineral assemblages by comparing laboratory estimates of conductivity with model conductivity profiles determined by GDS. In this way GDS studies of conductivity add to the inference of seismology, evidence of upper mantle rock samples and theories of the Earth's formation which allow speculation on the composition of the mantle.

Conductivity and temperature are related properties and assuming a particular mantle composition a derived conductivity model may be used to estimate the temperature variation with depth, eg. Banks (1969). This is potentially a very useful means of estimating mantle temperature. However, due to the non-uniqueness of conductivity models there will always be an element of uncertainty in addition to the error bounds of the conductivity-temperature relation derived by laboratory experiments.

Determining the composition of the mantle is an extremely complex problem as there are a large number of assumptions made and uncertainties in all available data. It should also be noted that the different disciplines which relate to the mantle draw on one anothers results sometimes without fully appreciating their accuracy and in addition circular arguments are used. For these reasons results concerning the mantle should be treated with caution. 


\section{Chapter 2}

\section{Magnetic Storms}

\subsection{Introduction}

For the most part time sampled measurements of the magnetic field at the surface of the Earth display a near regular behaviour governed by the periodic orbits of the Earth about the sun, the Earth's spin about its own axis and the motion of the moon. Irregularities, probably due to random events in the magnetosphere and ionosphere, are always present to corrupt magnetic measurements, however, sometimes other highly energetic signals interrupt the mainly periodic record. Disturbances known as geomagnetic storm-time variations may cause deviations of hundreds of nanoteslas ( $\mathrm{nT}$ ) in the magnetic components, the maximum departure from the pre-storm baseline usually occurs within ten hours from the commencement of the event. Magnetic storms occur virtually instantaneously at all points on the Earth and therefore they may be defined with respect to Universal Time (UT). Figure 2.1 shows how the storm of 17 th April 1965 affected the $X, Y$ and $Z$ magnetic components, note the components are defined with respect to geomagnetic co-ordinates (Section 4.2.2) as are the geographical terms used below. There follows a discussion of the temporal and spatial features of geomagnetic storm-time variations.

Every magnetic storm is different though certain common characteristics are usually present. A rapid increase in the $X$ component normally marks the beginning of a magnetic storm, this is called the sudden storm commencement and is denoted ssc. This is shown at the arbitary time of $\mathbf{3 5 0}$ hours in Figure 2.1. The second characteristic of a storm is the initial phase which is a short period of time during which the elevated $X$ component is retained, however, it is not a feature of all storms. The main phase then follows which sees a significant $X$ component decrease in magnitude occuring two to ten hours after the ssc, taking a few hours to complete. A corresponding change in 
Figure 2.1. Magnetograms showing the Magnetic Storm of 17th April 1965 recorded at Swider, Poland (swi).
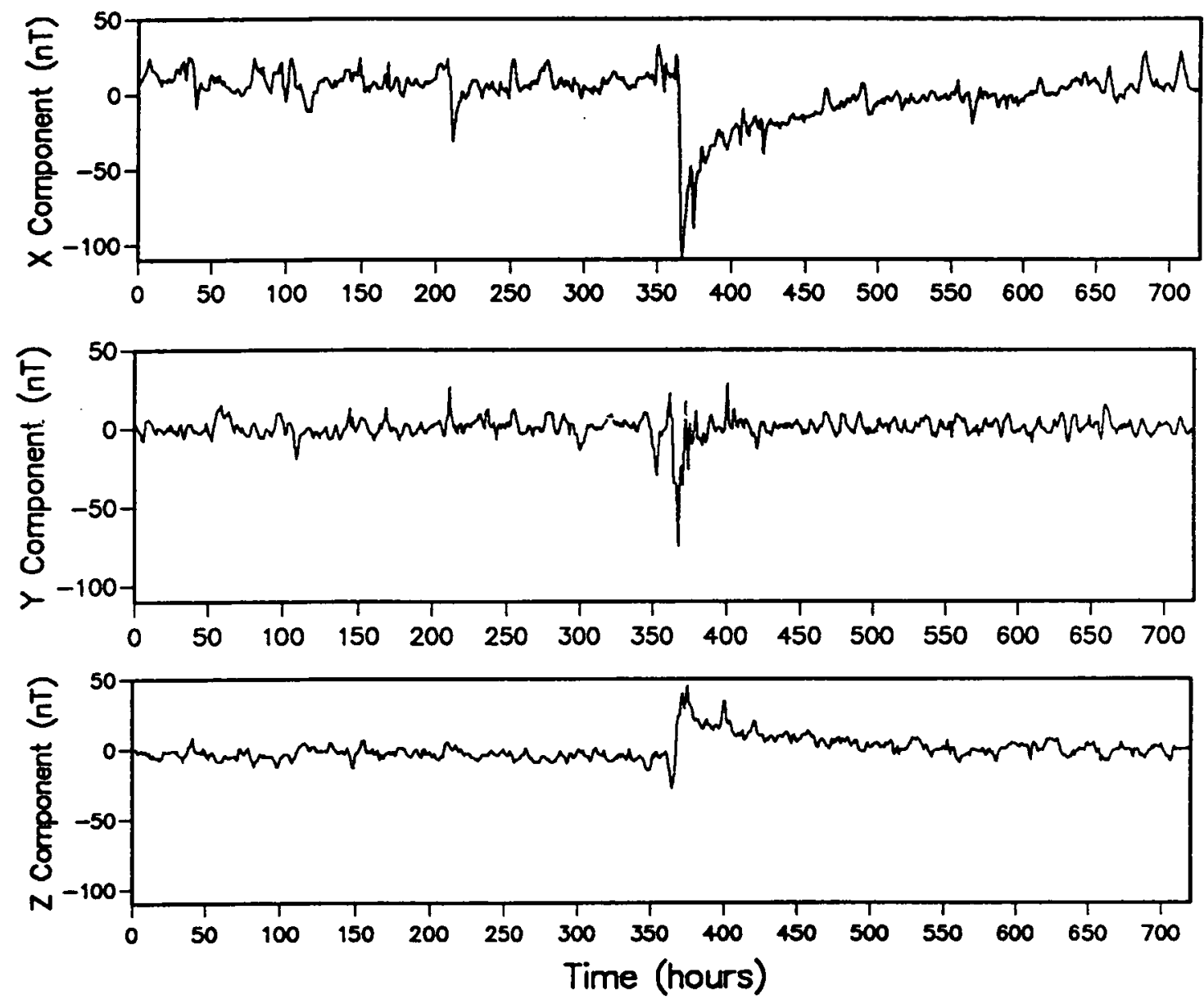
the magnitude of the vertical component takes place, the deviation is postive in the northern hemisphere and negative south of the geomagnetic equator, $Z$ is measured positive downwards. The final stage of a magnetic storm is called the recovery phase. During this period the field gradually reverts to the pre-storm level and usually takes several days, the form of the recovery is approximately exponential, see Figure 2.1. Throughout this discussion of the temporal characteristics the $Y$ component has not been mentioned. Figure 2.1 shows that the $Y$ component is influenced by the magnetic storm but does not show the general features described above. Thus the $Y$, eastwards, component does not appear to record the phenomena of magnetic storms accurately apart from displaying a short lived 'hiccup' while the storm is most energetic.

The magnitude of the disturbance due to a magnetic storm should also be considered with respect to position on the Earth's surface. In general the $X$ component main phase depression is greatest at the geomagnetic equator and decreases towards the poles. In comparison the magnitude of the deviation in the $Z$ component is virtually zero at the equator but increases as the poles are approached. As mentioned before the departure in $Z$ is such that the field is elevated in the northern hemisphere but depressed at southern latitudes.

In the plots of Figure 2.2 the power spectra for the previously discussed magnetograms are shown. For all three components the power generally falls off with increasing frequency to the Nyquist frequency of $12 \mathrm{cpd}$ or $0.5 \mathrm{cph}$. In the case of the $X$ and $Z$ components there is a great amount of power at the lowest frequencies, below 0.5 cpd, this is due to the long period recovery phase. As the $Y$ trace of Figure 2.1 did not show such a time of recovery there is no increased power at these low frequencies in the $Y$ power spectrum.

Although not shown in Figure 2.2 there are periodic variations associated with geomagnetic disturbances. There is a disturbed contribution to the daily variation due to substorms, see below. A quasi-periodic variation of 27 days, with harmonics, is present on the analysis of longer time intervals and this is thought to be related to 'coronal holes' in the sun's atmosphere which allow the escape of unusually high velocity solar wind (Section 2.2). The increased solar wind is experienced according to the apparent rotation of the sun which is 27 days viewed from the Earth. Considering even longer periods storms are found to be more common during the equinox seasons, giving a semi-annual periodicity, and there is some correlation between levels of disturbance and the sunspot cycle, to produce an eleven year variation.

Various terms have been adopted to refer to different geomagnetic signals. The average magnetic field recorded over some preferably evenly spaced and mid-latitude observatories results in what is known as the Dst field (disturbance, storm-time), a 
method of calculation is found in Sugiura (1964). This average field is therefore large when geomagnetic variations envelope the entire Earth simultaneously, i.e. for Universal Time phenomena. As magnetic storms are functions of UT the ssc may be used as a common origin for all locations, thus the name 'geomagnetic storm-time variations'. The hourly values of $D s t$ are sometimes called the $D s t$ index, alternatively the index may refer to an average over three hour intervals. If the Dst field is subtracted from the recording at each individual observatory the residual is Local Time (LT) dependent, i.e. of fundamental frequency $1 \mathrm{cpd}$, and is termed $D S$ (disturbance,solar). The average LT dependent variation, i.e. daily or diurnal part, found over a number of days is denoted $S D$. During selected days of quiet geomagnetic activity the average daily variation is called $S q$ (solar,quiet). The daily signal has been 'removed' in Figure 2.1 to show the storm more clearly, however, original magnetograms may be found in Figure A.2.

As well as there being disturbances known as storms there are also disturbances called substorms. These events do not have the characteristic morphology of storms but appear less ordered in their development with time. Substorms are predominately confined to latitudes near the auroral zones ( $\pm 68^{\circ}$ to $\pm 75^{\circ}$ geomagnetic latitude) and have quite a different spatial form to storms. However, substorms do have an affect at lower latitudes where variations of some tens of $\mathrm{nT}$ occur known as magnetic bays, at the higher latitudes substorms are commonly of a few hundreds of nT. Substorms are related to the phenomenon of aurora, the 'northern lights', which is sometimes observed over Britain during very intense substorms.

\subsection{The Earth's Magnetic Environment}

With increasing distance from the Earth internal sources other than the main dipole field completely diminish. However, the simple bar magnet magnetic field model does not represent the structure of the magnetic field external to the Earth as the interaction with the solar wind must be considered. The solar wind is a stream of high energy ionised particles, or plasma, that is constantly emitted from the sun. It behaves as a perfect conductor and carries with it magnetic field from the sun to the Earth, this is given the term interplanetary magnetic field (IMF). The solar wind is variable making the magnetic environment surrounding the Earth quite active.

Although the Earth's external magnetic field is never stationary the following overall picture describes the main features as they are presently understood. The result of the incident solar wind is to compress the Earth's magnetic field lines on the sunward side while the magnetic field lines in the shadow are pulled out into a tail, the magnetotail. The region confining the Earth's magnetic field is called the magnetosphere and 
Figure 2.3. The Magnetic Environment of the Earth. After Langel (1987).

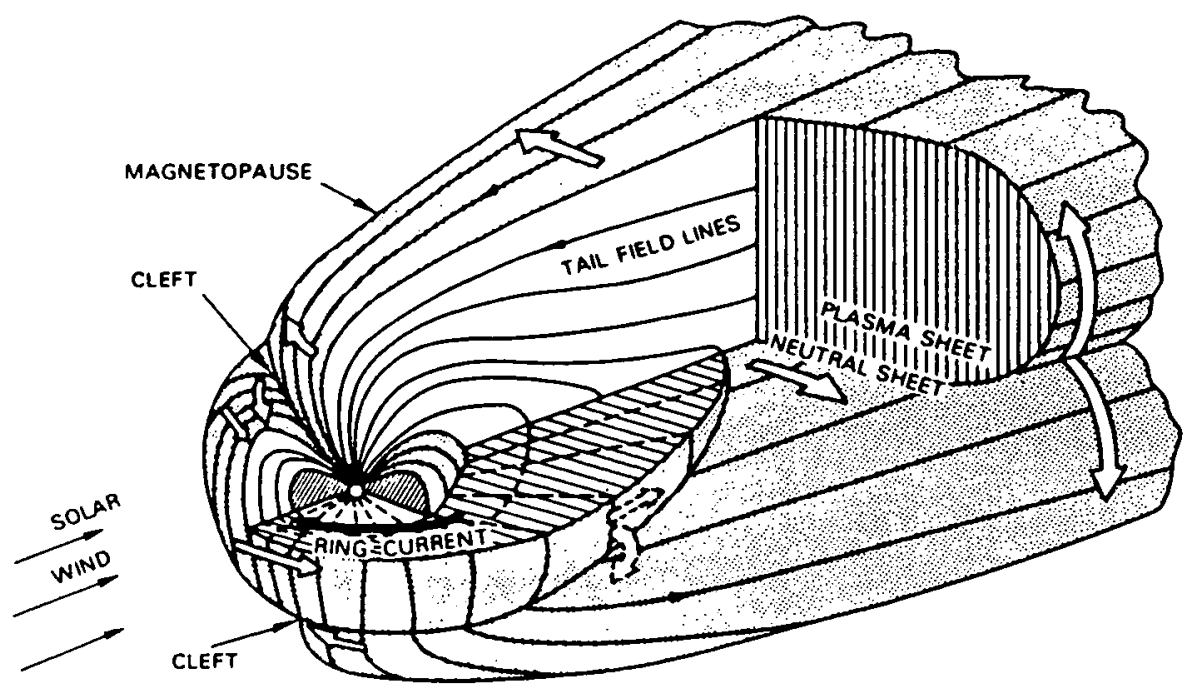

its asymmetric shape may be observed in Figure 2.3. The magnetosphere is taken to be the volume that the solar wind does not penetrate, its boundary is called the magnetopause. However, particles of the solar wind may enter the magnetosphere through the cleft. As the solar wind moves past the Earth electric currents flow at the magnetopause and across the centre of the magneto-tail, labelled neutral sheet in Figure 2.3. Within the magnetosphere currents are also generated to form a ring current that circles the Earth in the geomagnetic equatorial plane at around three Earth radii. Much nearer to the Earth the conducting upper atmosphere, the ionosphere, is another region with electric currents. There is some linkage between magnetospheric and ionospheric current systems at the auroral zones through currents that are aligned with the lines of magnetic field.

\subsection{Storm Generation Theory}

The precise mechanism which results in a magnetic storm is not completely known. The discussion that follows is based on the papers of Nishida (1978) McPherron (1979).

At a time of increased solar wind the magnetosphere becomes compressed causing 
the increase in the $X$ component observed at the ssc. The magneto-tail is also made to implode due to the higher than usual pressure. This event pushes plasma into the magnetosphere providing particles to carry current. Within the magnetosphere a trapping region exists in which ions are restricted and ions outside may not penetrate. This region is dependent on the electric field and so if conditions change to make the trapping region larger more ions may be retained. If this enlargement coincides with the injection of plasma from the magneto-tail there is a considerable increase in trapped charged particles within the magnetosphere. The gradient in the magnetic field of the Earth then causes the ions in the trapping region to drift, positive west and negative east. Thus a westward current is produced, this is the ring current, see Figure 2.3.

The enhancement of the ring current corresponds to the main phase of a magnetic storm, the westward current opposes the Earth's own field and so explains the decrease in $X$ and numerical increase in the $Z$ component. At this stage it is probable that the ring current is not complete but only partially encloses the Earth. However, once the main phase is complete the ring current is more symmetric creating a uniform southward directed magnetic field similar to that produced by a solenoid. The completed ring current does not though persist at the magnitude it has obtained but due to charge exchange with neutral hydrogen atoms undergoes an exponential decay.

This brief description of the theoretical generation of magnetic storms paints a relatively simple picture. However, it should be appreciated that this is a theory which is not supported in entirety by all the researchers in the field. An article by Matsushita (1975), directed towards those using magnetic variations for EM induction studies, concludes that the simple ring current does not accurately describe the conditions responsible for storms but points towards a more complex system of partial ring currents and field-aligned currents. This view involves a close relationship between storms and substorms. Kamide (1991) examined the question as to whether storms are summations of substorms but was not able to resolve the problem. Thus although the discussed theory may not be totally correct it nevertheless does account for the observations and provides some idea as to the processes responsible for magnetic storms.

\subsection{Application to Geomagnetic Deep Sounding}

\subsubsection{General}

Geomagnetic storms are transient features with an initial burst of high frequency energy followed by a gradual recovery to the pre-storm state, e.g. Figure 2.1. Transient signals are made up of a wide range of frequencies and therefore, because of the skin 
depth relation (Equation 3.21), are amenable to the problem of determining electrical conductivity against depth. The method of determining electrical conductivity at depths extending into the Earth's mantle is given the name geomagnetic deep sounding (GDS). As well as magnetic storms the diurnal variation $S q$ may also be used to investigate the conductivity of the Earth but this source is limited in frequency range and therefore does not relate to such a large depth range as storm variations. Magnetic bays and the lunar variation are other sources but again these are not able to induce currents at the required depth and suffer from poor signal power. The frequencies considered in this work extend between 0.03 and $1.0 \mathrm{cpd}$ which correspond to depths of around 400 to $1000 \mathrm{~km}$.

The spatial structure of the storm field has been discussed above (Section 2.1) and is considered in Section 6.3 where it is concluded that a sole $P_{1}^{0}$ spherical harmonic is a fair approximation. Thus magnetic storms are suitable for global studies as the source field may be assumed to have a simple form and encompasses the whole Earth.

The Earth's response to magnetic variations is dependent both on the morphology of the source field and the distribution of conductivity. If the conductivity were to vary with latitude and longitude within the Earth, as well as radially, a $P_{1}^{0}$ source would induce fields of other modes. This presents an extremely complex problem to solve. However, it is reasonable to assume that the Earth is spherically symmetric below 400 $\mathrm{km}$ and that the conductivity varies only with the radial distance from the centre, i.e. reduce to a one dimensional problem. This assumption is based on seismological evidence and is also supported by cosmological theories of the Earth's formation, which were mentioned in Section 1.2.2 and 1.2.3. However, recently seismic tomographic studies, reviewed in Anderson (1989), have suggested that there are small lateral changes in the seismic velocity within the upper and lower mantle and therefore if there is any relationship between seismic velocity and conductivity there may also be some latitude/longitude conductivity variation. Indeed some recent work on electromagnetic studies (Schultz and Larsen, 1987; Petersons and Anderssen, 1990; Schultz and Larsen, 1990; Schultz, 1990) has shown that geographical differences in the conductivity depth profile are suggested. However, from the overall evidence for the properties of the mantle any lateral variations are almost certainly considerably smaller than the vertical conductivity gradient.

Above $400 \mathrm{~km}$ lateral conductivity changes are significant as the magnetotelluric method has shown. The greatest contrast in conductivity is that between oceans and the continental land masses. It is therefore also assumed that conductivity variations near to the surface and within the topmost $400 \mathrm{~km}$ do not affect the electromagnetic response of the mid-mantle. Calculation of the response for coastal, island and inland 
observatories (Grafe, 1963) confirmed that the presence of oceans does not affect the response at storm frequencies, though conversely Roberts (1984) found that his work did indicate different response functions for observatories positioned at different locations with respect to oceans. Additionally Pecova et al. (1980) found that for observatories within $200 \mathrm{~km}$ of oceans the response at 27 days period was affected. Thus even at the periods important in storm studies, greater than 1 day, the distribution of the oceans may have a bearing on results.

In this study, however, the assumptions have been made that the mid-mantle conductivity depends only on radial distance and that near surface lateral conductivity differences do not appreciably affect the response at storm frequencies. These assumptions are necessary to make the problem manageable and have been used by previous workers, nevertheless it should be borne in mind that they may be violated to some extent as some recent research has shown.

\subsubsection{Previous Studies}

The first use of magnetic storms for GDS was carried out by Chapman and Whitehead (1922), a further study was conducted by Chapman and Price (1930) and in 1939 Lahiri and Price included magnetic storms to produce two models which they proposed as limits to the conductivity depth profile. Since then there have been a number of analyses of storms: Benkova (1953), Rititake and Sato (1957), Anderssen and Seneta (1969) and Anderssen et al. (1970), though these did not go on to relate the findings to the conductivity distribution of the Earth. Models for the electrical conductivity profile were proposed by Rikitake (1966), Jady (1975) and Devane(1977) and also Hobbs (1987) which was based on the work of Marshall (1980). This body of work indicates increasing conductivity with depth as shown by Figure 1.3. Rikitake and Jady suggested models with a step to higher conductivity at approximately 400 and $500 \mathrm{~km}$ respectively.

It was stated in the previous section that some work has indicated that the conductivity of the mid-mantle is not purely a function of depth and it may be that this complication has contributed to the different models proposed for the global depth profile. However, by undertaking an investigation based on a large database of storms from different sets of magnetic observatories it is the aim of this present study to see whether the electromagnetic response of the Earth can be defined sufficiently to determine an average global conductivity model for the mid-mantle. 


\subsubsection{Method}

There follows an account of the method used in the analysis of individual magnetic storms. An overview of the techniques employed by some researchers, mainly Russian, is given by Rokityansky (1982; p112).

Three component magnetic field mesurements from some set of observatories constitute the original data to be used to yield information on the electrical conductivity of the Earth's interior. Major stages in the treatment of the data are Fourier Transformation (FT) and spherical harmonic analysis (SHA). Subtraction of the periodic daily variation and smoothing of the time series may be applied before the above steps. It is then possible to separate the internal and external parts of potential (Sections 3.3.3 and 6.5) and determine the response (Section 7.1.1) as a function of frequency. The overall plan for the analysis of an individual storm is presented in Figure 2.4.

To determine a conductivity depth profile an estimate of the response must be known for a range of frequencies. Therefore Fourier Transformation of the time series is necessary at some point in the analysis. A purely time domain approach may be pursued (Marshall, 1980), and this does allow the radius of a perfectly conducting inner 'core' to be estimated. However, this is primarily intended for comparing different storm analyses (Jady et al., 1979).

The scheme outlined in Figure 2.4 shows two alternative routes leading to the response function. Either FT or SHA may be carried out first as SHA is equally valid on the Fourier coefficients or time series. Rokityansky (1982) suggests that the analysis should be carried out both ways round to allow the results to be compared and reliability assessed. The SHA of Marshall in the time domain and subsequent FT carried out by Hobbs is an example of route $B$ of Figure 2.4, however, the work of the following chapters takes route $A$, the reasons for this are given in Section 5.1.1. 
Figure 2.4. The Analysis Strategy.

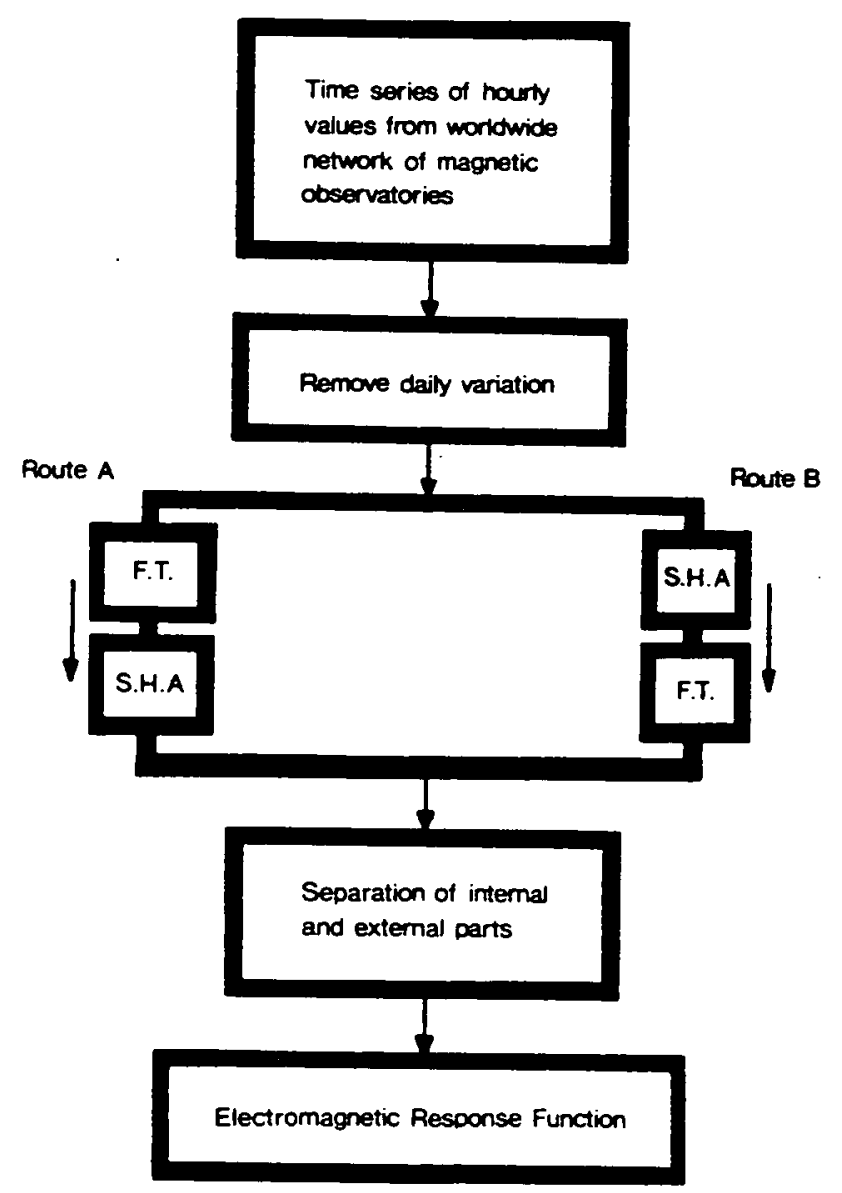




\section{Chapter 3}

\section{Theoretical Background}

\subsection{Electromagnetic Theory}

\subsubsection{The Basic Equations}

An electromagnetic field may be described by the vectors
E Electric Field,
D Electric Displacement,
B Magnetic Induction,
H Magnetic Field.

Definitions of these four vector quantities may be found in Grant and Phillips (1982). The terms used for these vectors are not consistent throughout the literature, those given above comply with the proposal of the LAGA Committee on Semantics (Hobbs, 1992). Thus the use of 'magnetic field' should be reserved for the vector $H$, however, it is common practice to refer $\mathbf{B}$ as the 'magnetic field' when discussing observatory measurements.

Using these vectors an electromagnetic field obeys the following four equations, named Maxwell's equations after the physicist James Clerk Maxwell.

$$
\begin{gathered}
\nabla \times \mathbf{H}=\mathbf{J}+\dot{\mathbf{D}} \\
\nabla \cdot \mathbf{D}=\boldsymbol{\rho} \\
\nabla \times \mathbf{E}=-\dot{\mathbf{B}} \\
\nabla \cdot \mathbf{B}=\mathbf{0}
\end{gathered}
$$


$\mathrm{J}$ is the current density and $\rho$ the charge density, a dot above a vector denotes partial differentiation with respect to time. For isotropic media

$$
\begin{aligned}
\mathbf{D} & =\varepsilon \mathbf{E} \\
\mathbf{B} & =\mu \mathbf{H} \\
\mathbf{J} & =\sigma \mathbf{E}
\end{aligned}
$$

where $\sigma$ is the conductivity, $\varepsilon$ the permittivity and $\mu$ the permeability.

\subsubsection{Electromagnetic Fields in an Ohmic Medium}

To gain insight to the electrical properties of the Earth it is necessary to first of all understand the behaviour of an electromagnetic field propagating within a material of known conductivity. Consider a medium with parameters $\sigma, \varepsilon$ and $\mu$ subject to a uniform plane electromagnetic wave. Then using Equations 3.5, 3.6 and 3.7, Maxwell's Equations 3.1 and 3.3 may be written

$$
\begin{gathered}
\nabla \times \mathbf{H}=\sigma \mathbf{E}+\varepsilon \dot{\mathbf{E}} \\
\nabla \times \mathbf{E}=-\mu \dot{\mathbf{H}}
\end{gathered}
$$

If Equation 3.6 is substituted into Equation 3.4 then

$$
\boldsymbol{\nabla} \cdot \mathbf{H}=\mathbf{0}
$$

It may be shown (Duffin, 1990; p349) that free charge in an ohmic material decays to zero exponentially. Therefore after some relaxation time, and using Equation 3.5, Equation 3.2 reduces to

$$
\boldsymbol{\nabla} \cdot \mathbf{E}=\mathbf{0}
$$

With typical geomagnetic freqencies the relaxation time may be ignored as the decay is effectively instantaneous. It is then possible to derive equations involving only $\mathbf{E}$ or $H$. Application of the $\nabla \times$ operation $^{1}$ to both sides of Equation 3.8 followed by substitution of Equations 3.9 and 3.10 gives

$$
\nabla^{2} \mathbf{H}-\mu \varepsilon \ddot{\mathbf{H}}-\mu \sigma \dot{\mathbf{H}}=\mathbf{0}
$$

\footnotetext{
${ }^{1}$ Applying the identity $\nabla \times \nabla \times=\nabla \nabla \cdot-\nabla^{2}$.
} 
Similarly using Equations 3.8, 3.9 and 3.11

$$
\nabla^{2} \mathbf{E}-\mu \varepsilon \ddot{\mathbf{E}}-\mu \sigma \dot{\mathbf{E}}=\mathbf{0}
$$

If $\mathbf{H}$ oscillates with angular frequency $\omega$ and is of amplitude $H$ it may be expressed in complex notation

$$
\mathbf{H}=H \exp [i \omega t]
$$

where $i=\sqrt{-1}$. Therefore

$$
\dot{\mathrm{H}}=i \omega \mathbf{H}
$$

and

$$
\ddot{\mathrm{H}}=i \omega \dot{\mathrm{H}}
$$

Therefore Equation 3.12 is equivalent to

$$
\nabla^{2} \mathbf{H}-i \omega \mu \varepsilon \dot{\mathbf{H}}-\mu \sigma \dot{\mathbf{H}}=0
$$

In a similar manner for $\mathbf{E}$

$$
\nabla^{2} \mathbf{E}-i \omega \mu \varepsilon \dot{\mathbf{E}}-\mu \sigma \dot{\mathbf{E}}=\mathbf{0}
$$

These two equations may be simplified by considering the relative sizes of the second and third terms. The magnitude of $\omega \varepsilon$ in relation to $\sigma$ determines whether the second or third term is dominant. This may be restated by comparing $\omega$ to $\sigma / \varepsilon$. For the Earth a very low estimate of conductivity is $10^{-3} \mathrm{Sm}^{-1}$. Taking $\varepsilon_{0}$ for $\varepsilon$ this gives $\sigma / \varepsilon \approx 10^{8} \mathrm{cps}$. This is far in excess of the frequency of geomagnetic variations under study, greatest frequency $\approx 10^{-5} \mathrm{cps}$, and so the second term of Equation 3.14 and 3.15 is of far less significance than the third. Thus

$$
\begin{aligned}
\nabla^{2} \mathbf{H}-\mu \sigma \dot{\mathrm{H}} & =0 \\
\nabla^{2} \mathbf{E}-\mu \sigma \dot{\mathrm{E}} & =\mathbf{0}
\end{aligned}
$$

The electric displacement has been neglected due to the slow variation of geomagnetic variations, referred as the quasi-static case. Equations 3.16 and 3.17 are examples of the diffusion equation. For a plane wave travelling in the $z$ direction the physical solution of Equations 3.16 and 3.17 are

$$
\begin{aligned}
& \mathbf{H}=\mathbf{H}_{\mathbf{0}} \exp [i(\omega t-\beta z)] \exp [-\alpha z] \\
& \mathbf{E}=\mathbf{E}_{0} \exp [i(\omega t-\beta z)] \exp [-\alpha z]
\end{aligned}
$$


where

$$
\alpha=\beta=\sqrt{\frac{\mu \sigma \omega}{2}}
$$

The quantity $1 / \alpha$ is called the skin depth $(\delta)$, it is usually assumed that $\mu$ is constant and equal to $\mu_{0}$ (eg. Roberts, 1982), thus

$$
\delta=\frac{1}{\alpha}=\sqrt{\frac{2}{\mu_{0} \sigma \omega}}
$$

The skin depth therefore gives the depth of penetration at which the wave is attenuated by a factor $1 / e$. For a source with energy at a range of freqencies the skin depth is larger for lower frequencies, thus deeper depths may be probed.

\subsubsection{Electromagnetic Fields in and around the Earth.}

It is desired to determine the variation of conductivity with depth into the Earth, therefore the above theory must be extended for variable $\sigma$. It should be noted that $\sigma$ is only considered to vary with depth, ie. the problem is restricted to one dimension. The quasi-static approximation is assumed so that displacement currents may be neglected and the permeability $\mu$ is taken as being constant and equal to $\mu_{0}$. Equations 3.8 and 3.9 are again operated on by $\nabla \times$ though $\sigma$ is now variable so that

$$
-\nabla^{2} \mathbf{H}=-\sigma \mu \dot{\mathbf{H}}+\frac{1}{\sigma} \nabla \sigma \times \nabla \times \mathbf{H}
$$

and

$$
\nabla^{2} \mathbf{E}=\sigma \mu \dot{\mathbf{E}}
$$

Equation 3.22 is difficult to solve, however, the solution of Equation 3.23 may be found within the Earth and then the magnetic field calculated using Equation 3.3.

The atmosphere immediately surrounding the Earth is virtually a non conductor, Equations 3.1 and 3.7 therefore imply

$$
\mathbf{H}=-\nabla \boldsymbol{\Omega}
$$

where $\Omega$ is the magnetic scalar potential. Furthermore Equations 3.4 and 3.6 mean that the above leads to

$$
\nabla^{2} \Omega=0
$$

which is Laplace's equation. The solution to Equation 3.23 (Stratton, 1941) has the 
three forms

$$
\begin{gathered}
\mathbf{T}=\nabla \times(\Psi r) \\
\mathbf{S}=\nabla \times \nabla \times(\Phi r) \\
\mathbf{U}=-\nabla \Omega
\end{gathered}
$$

which are the toroidal, poloidal and irrotational vector fields respectively. In a nonconducting region such as that surrounding the Earth only the irrotational solution exists. This is Laplace's equation which was given above (Equation 3.25). Inside a conductor the field is given by the sum of the toroidal and poloidal fields. It may be assumed that there are no cross surface currents flowing from the Earth and therefore the electric field must be completely toroidal inside the Earth. The toroidal $\mathbf{E}$ field solution may then be substituted into Equation 3.23 and solved by separation of variables in terms of spherical harmonics (Section 3.3.1) and a function of the radial distance. With this solution inside the Earth it may be matched to the solution outside the Earth by the boundary conditions ${ }^{2}$.

The solution of Equation 3.25 in spherical co-ordinates takes the form

$\Omega\left(r, \theta^{\prime}, \phi^{\prime}\right)=a \sum_{n=1}^{\infty} \sum_{m=0}^{n}\left\{\left(g_{n}^{m} \cos m \phi^{\prime}+h_{n}^{m} \sin m \phi^{\prime}\right)\left(\frac{r}{a}\right)^{-(n+1)}+\left(g_{n}^{\prime m} \cos m \phi^{\prime}+h_{n}^{\prime m} \sin m \phi^{\prime}\right)\left(\frac{r}{a}\right)^{n}\right\} P_{n}^{m}\left(\cos \theta^{\prime}\right)$

where $g_{n}^{m}, h_{n}^{m} g_{n}^{\prime m}, h_{n}^{\prime m}=$ coefficients

$$
\begin{array}{lll}
r & =\text { radial distance from Earth's centre } \\
a & =\text { radius of Earth } \\
\theta^{\prime} & =\text { geomagnetic co-latitude } \\
\phi^{\prime} & =\text { geomagnetic east-longitude } \\
P_{n}^{m}\left(\cos \theta^{\prime}\right) & =\text { associated Legendre polynomial of degree } n \text { and order } m .
\end{array}
$$

The coefficients $g_{n}^{m}, h_{n}^{m}$ correspond to a potential function which decreases with increasing $r$ and so represent internal sources. Conversely the coefficients $g_{n}^{\prime m}, h_{n}^{\prime m}$ correspond to an increasing potential function with increasing $r$ and so represent external sources.

Substituting Equation 3.26 in Equation 3.24 and using relation 3.6 the three components of the magnetic field may be found

$$
X=\left[\frac{\mu_{0}}{r}\left(\frac{\partial \Omega}{\partial \theta^{\prime}}\right)\right]_{r=0}
$$

\footnotetext{
${ }^{2}$ The boundary conditons may be found in standard electromagnetism textbooks such as Grant and Phillips (1982) or Duffin (1990).
} 


$$
\begin{gathered}
Y=-\left[\frac{\mu_{0}}{r \sin \theta^{\prime}}\left(\frac{\partial \Omega}{\partial \phi^{\prime}}\right)\right]_{r=a} \\
Z=\mu_{0}\left(\frac{\partial \Omega}{\partial r}\right)_{r=a}
\end{gathered}
$$

Therefore

$$
\begin{gathered}
X=\sum_{n=1}^{\infty} \sum_{m=0}^{n}\left\{\left(g_{n}^{m} \cos m \phi^{\prime}+h_{n}^{m} \sin m \phi^{\prime}\right)+\left(g_{n}^{\prime m} \cos m \phi^{\prime}+h_{n}^{\prime m} \sin m \phi^{\prime}\right)\right\} \frac{\partial}{\partial \theta^{\prime}}\left(P_{n}^{m}\left(\cos \theta^{\prime}\right)\right) \\
Y=\sum_{n=1}^{\infty} \sum_{m=0}^{n}\left\{\left(m g_{n}^{m} \sin m \phi^{\prime}-m h_{n}^{m} \cos m \phi^{\prime}\right)+\left(m g_{n}^{\prime m} \sin m \phi^{\prime}-m h_{n}^{\prime m} \cos m \phi^{\prime}\right)\right\} \frac{P_{n}^{m}\left(\cos \theta^{\prime}\right)}{\sin \theta^{\prime}} \\
Z=\sum_{n=1}^{\infty} \sum_{m=0}^{n}\left\{-(n+1)\left(g_{n}^{m} \cos m \phi^{\prime}+h_{n}^{m} \sin m \phi^{\prime}\right)+n\left(g_{n}^{\prime m} \cos m \phi^{\prime}+h_{n}^{\prime m} \sin m \phi^{\prime}\right)\right\} P_{n}^{m}\left(\cos \theta^{\prime}\right)
\end{gathered}
$$

These equations describe the magnetic components over the surface of the Earth derived from Laplace's equation for the potential. Analyses of the main field reveal the dominance of the internal source of the geodynamo. The background field originating within the Earth may, however, be removed and ignored in the analysis of geomagnetic storms. Equations 3.27, 3.28 and 3.29 may then be applied to the residual $X, Y, Z$ time series to determine the coefficients associated with the magnetic disturbance. The three equations are applicable to the magnetic components given in the time or frequency domain.

\subsection{Fourier Transformation}

\subsubsection{The Discrete Fourier Transform}

A continuous function that varies in time may be represented in terms of its frequency content by application of the Fourier transform. The Fourier Transform $G(f)$ of a function $g(t)$ is defined as

$$
G(f)=\int_{-\infty}^{\infty} g(t) \exp [i 2 \pi f t] d t
$$

where $t$ is time, $f$ is frequency in cycles per unit time and $i=\sqrt{-1}$. The inverse Fourier transform carries out the operation in the reverse sense to regain the variation in time. 
Thus

$$
g(t)=\int_{-\infty}^{\infty} G(f) \exp [-i 2 \pi f t] d f
$$

Together, Equations 3.30 and 3.31 are known as a transform pair, this may be indicated by the notation $g(t) \leftrightarrow G(f)$. However, time signals may only be recorded over a finite duration and are invariably given in digital form. An approximation of the above transform pair must therefore be used in practice. Equations 3.32 and 3.33 form the discrete transform pair. To transform from time to frequency

$$
G_{m}=\sum_{l=-N / 2}^{N / 2} g_{l} \exp \left[i 2 \pi \frac{l m}{N}\right], \quad m=-N / 2, \ldots,-1,0,1, \ldots, N / 2
$$

and to revert to the time domain

$$
g_{l}=\frac{1}{N} \sum_{m=-N / 2}^{N / 2} G_{m} \exp \left[-i 2 \pi \frac{l m}{N}\right], \quad l=-N / 2, \ldots,-1,0,1, \ldots, N / 2,
$$

where $g_{l} \equiv g\left(t_{l}\right)$ and $G_{m} \equiv G\left(f_{m}\right)$ and the sampling interval is one unit of time. The indices of time or frequency have been given between $-N / 2$ and $N / 2$ however, in practice the limits are 0 to $N-1$ requiring a shift of the axis. This alters phase angles of the transform but not magnitude.

To evaluate the discrete Fourier transform (DFT) an algorithm known as the fast Fourier transform (FFT) is used. The development of the FFT is outlined in Kanasewich (1981). Greater efficiency is achieved by the FFT due to a factorisaton scheme which significantly reduces computational effort. The original FFT algorithm relied on the number of data points $(N)$ being a power of 2 . However, procedures now exist allowing products of any other factors.

This study is concerned with time series analysis, however, Fourier methods are equally important in the investigation of sinusoidal signals in spatial data. The work of Stewart (1990) is an example.

\subsubsection{Window Functions}

As any dataset is restricted to a finite length the Fourier transform is an estimate of the transform observed if the data were to extend to infinity in either direction. The data sequence is assumed to observe a fundamental periodicity equal to the data length. However, a signal of a frequency that is not a harmonic of the fundamental frequency will be discontinuous at the ends of the time series. This will hinder estimation of the magnitude of the Fourier coefficient and detection of the component Fourier frequency. 

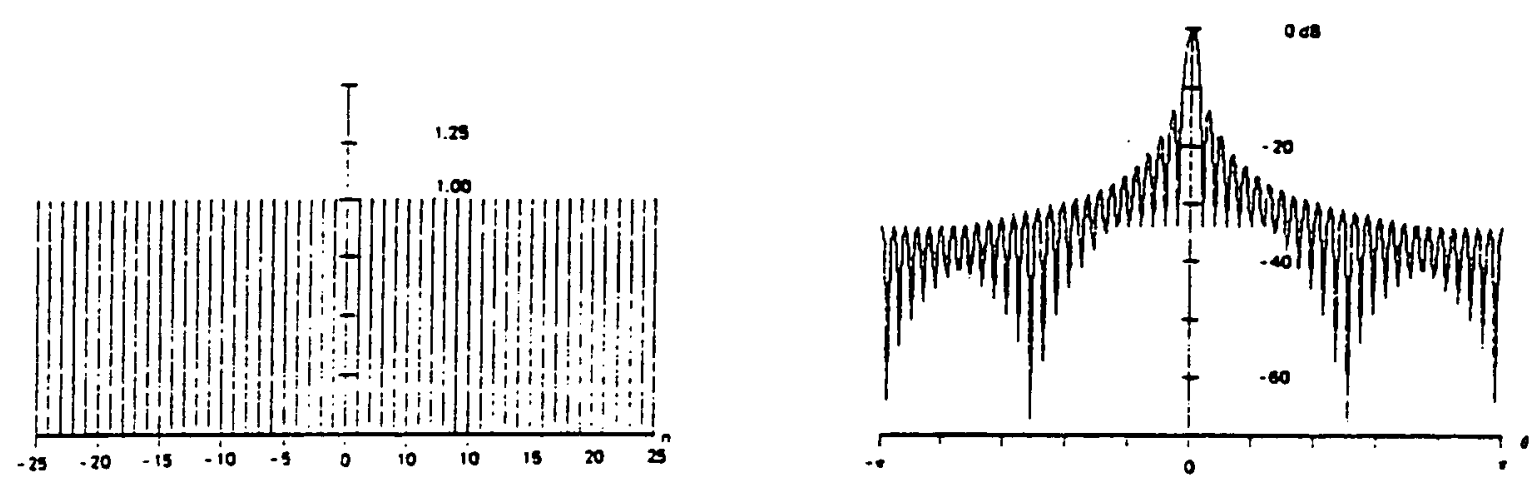

Figure 3.1. The Dirac Comb function and log-magnitude of its Fourier Transform. After Harris (1978).

Energy is distributed throughout the entire frequency range due to the discontinuity rather than solely at the desired point along the frequency axis. This phenomenon due to the Fourier transformation of finite-duration records is called leakage. In addition data values are observed at disrete intervals of time. The sampling rate governs the spacing of points in the frequency domain and thus the manifestation of leakage is dependent on sampling though it is not the cause.

Measuring a finite time sequence is equivalent to multiplying the infinite sequence by a boxcar function equal in length to the time of observation. The boxcar function is defined

$$
w(t)= \begin{cases}1 & \text { if }-N / 2 \leq t \leq N / 2 \\ 0 & \text { otherwise }\end{cases}
$$

If $w(t)$ is replaced by the discrete form $w\left(t_{l}\right)$ Equation 3.34 becomes a sequence of Dirac delta functions known as a Dirac comb. The Dirac comb and its Fourier Transform are shown in Figure 3.1. This is the data window used implicitly when taking a finite length of digital data. The term 'data window' is used to refer to the window in the time domain.

To explain the effects of data windows it is necessary to introduce the operation of convolution. Mathematically the convolution $z\left(t_{L}\right)$ between two discrete functions 
$\left[x\left(t_{l}\right)\right]_{l=0,1, \ldots, N}$ and $\left[g\left(t_{l}\right)\right]_{l=0,1, \ldots, M}$ is written

$$
z\left(t_{L}\right)=(x * g)_{L}=\sum_{k=0}^{M} x\left(t_{L-k}\right) g\left(t_{k}\right)
$$

where $L=0,1, \ldots, M+N$. It is a process of multiplying one function by a lagged and reversed copy of the other, the multiplication between the functions is carried out for each lag. It is used extensively in geophysical data processing and is explained in a number of textbooks including Kanasewich (1981). The equivalence of multiplication in the time domain with convolution of the Fourier transforms in the frequency domain is proved in the appendix of Harris (1978), the relationship may also be applied in the reverse sense. Therefore the result of Fourier analysis of a finite discrete data sample is the convolution of the Fourier transform of the infinite time series with the Fourier transform of the Dirac Comb function. The nature of the transform is shown in Figure 3.1. The oscillations either side of the main peak are called sidelobes which show how the power 'leaks' and is distributed throughout the frequency space.

The cause of leakage has been explained in terms of the discontinuous signals not periodic within the finite record length. The order of discontinuities can, however, be reduced by changing the shape of the data window. If a data window is made to smoothly decrease to zero at the limits of the observed data sequence sharp discontinuities are removed. An example of such a data window is the Hanning window shown in Figure 3.2. It is a member of a family of windows defined in terms of $\cos ^{\alpha}(l / N \pi)$ where $\alpha$ is an integer which equals 2 for the Hanning window, and $l$ and $N$ are as above 3. Thus the window gradually decreases to zero at the boundaries when $l=-N / 2$ and $l=N / 2$. The window is either applied by multiplication of the time series by the data window or by convolution of the Fourier coefficients with the transform of the window. Harris (1978) contains a full description of the Hanning and a selection of other windows. In the frequency domain the use of such a window reduces the size of the sidelobes contributing to the leakage. However, a decrease in leakage is achieved at the expense of the resolution of component frequencies as the main lobe is larger in width than for the Dirac comb window (Figure 3.1).

Attempts at deriving the optimum balance between reducing bias, due to leakage, and maintaining resolution have lead to the use of various different window functions. To assess the relative merit of a number of distinct windows Table 1 of Harris (1978)

\footnotetext{
${ }^{3}$ The trigonometric term depends on the definition of the interval of interest which is taken to be between $-N / 2$ and $N / 2$ here.
} 

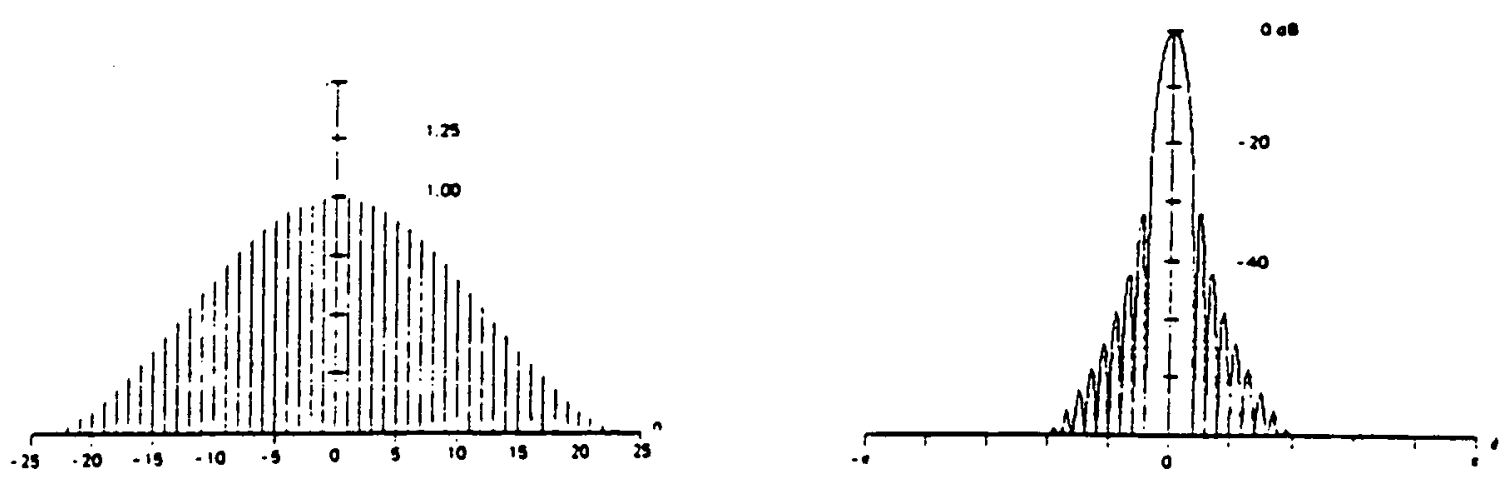

Figure 3.2. The Hanning data window $\left(\cos ^{2}(l / N \pi)\right)$ and $\log$-magnitude of transform. After Harris (1978).

should be consulted. A novel approach to the problem has been formulated by Thomson (1982) who proposed the use of multiple window functions. By combining the results of different windows it is aimed to bring out the advantageous characteristics of each individual window and so achieve both low bias and high resolution of the Fourier coefficients.

\subsection{Spherical Harmonic Analysis}

\subsubsection{Introduction}

A time series, or sequence of values distributed in space, may be represented by a series of Fourier coefficients, as described in the previous section. In a similar way mesurements over a sphere may be modelled in terms of an expansion of spherical surface harmonic functions. These functions arise from the solution of Laplace's equation in spherical polar co-ordinates and are orthogonal.

A function $f(\theta, \phi)$ defined over a sphere may be expanded in terms of spherical surface harmonics as

$$
f(\theta, \phi)=\sum_{n=0}^{L} \sum_{m=0}^{n}\left(a_{n}^{m} \cos m \phi+b_{n}^{m} \sin m \phi\right) P_{n}^{m}(\cos \theta)
$$


where $a_{n}^{m}, b_{n}^{m} \quad=$ spherical harmonic coefficients (shc's)

$\theta \quad=$ co-latitude

$\phi \quad=$ east-longitude

$P_{n}^{m}(\cos \theta)=$ associated Legendre polynomial of degree $n$ and order $m$.

The above equation is an approximation to the infinite sum of spherical harmonic functions, being truncated at degree and order $L$. A normalisation factor, such as that defined by Schmidt (Chapman and Bartels, 1940), is usually applied to the associated Legendre polynomial. Making use of the property of orthogonality the coefficients of the expansion may be determined as in Fourier Analysis. Thus

$$
\left\{\begin{array}{l}
a_{n}^{m} \\
b_{n}^{m}
\end{array}\right\}=C \int_{0}^{2 \pi} \int_{0}^{\pi} f(\theta, \phi) P_{n}^{m}(\cos \theta)\left\{\begin{array}{c}
\cos m \phi \\
\sin m \phi
\end{array}\right\} \sin \theta d \theta d \phi
$$

where $C$ is the normalisation factor. If $f(\theta, \phi)$ is known over a finite evenly distributed grid the spherical harmonic coefficients may only be evaluated up to a limit determined by the grid spacing. The determination of the coefficients in this manner is called Spherical Harmonic Analysis (SHA). However, geomagnetic observatories are not evenly distributed over the Earth's surface, the system is no longer orthogonal, therefore an approximation to SHA must be carried out. This is achieved by an inverse method such as Least Squares though other techniques have also been applied to the problem.

\subsubsection{SHA using Least Squares}

The method of Least Squares (LS) is a means of determining the parameters of a model chosen to represent a set of data. A simple example of the use of LS is to fit a straight line to some measurements which are expected to observe a linear trend. The application of LS to this problem will result in an estimate of the gradient and intercept parameters which define the model line. The method may be extended to fit any model curve to the observed data. The problem of concern here is to determine the coefficients of the spherical harmonic model that fit measurements of the magnetic field recorded over the Earth's surface.

The models of interest are given by Equations 3.27, 3.28 and 3.29. To describe the LS method for estimating the spherical harmonic coefficients consider Equation 3.27 which may be written

$$
X_{M}=\sum_{n=1}^{L} \sum_{m=0}^{n}\left\{a_{n}^{m} \cos m \phi^{\prime}+b_{n}^{m} \sin m \phi^{\prime}\right\} \frac{\partial}{\partial \theta^{\prime}}\left(P_{n}^{m}\left(\cos \theta^{\prime}\right)\right)
$$


where $X_{M}=X$ component derived from potential,

$a_{n}^{m} \quad=g_{n}^{m}+g_{n}^{\prime m}$

$b_{n}^{m}=h_{n}^{m}+h_{n}^{\prime m}$,

$L \quad=$ degree of expansion.

The other terms are as defined for Equation 3.27. It is desired to determine the coefficients $g_{n}^{m}, h_{n}^{m}, g_{n}^{\prime m}$ and $h_{n}^{\prime m}$.

The observations will invariably deviate from $X_{M}\left(\theta^{\prime}{ }_{i}, \phi_{i}^{\prime}\right)$ due to assumptions made in the model, i.e. early truncation, and measurement error. The difference between the observed values $X_{O}\left(\theta_{i}^{\prime}, \phi_{i}^{\prime}\right)$ and the model may be found for each observation point. This residual or prediction error $\varepsilon\left(\theta^{\prime}{ }_{i}, \phi_{i}^{\prime}\right)$ is given by

$$
\varepsilon\left(\theta_{i}^{\prime}, \phi_{i}^{\prime}\right)=X_{O}\left(\theta_{i}^{\prime}, \phi_{i}^{\prime}\right)-X_{M}\left(\theta_{i}^{\prime}, \phi_{i}^{\prime}\right)
$$

The model parameters should be found so that the model agrees as closely as possible with the observations. One scheme is to find the model coefficients which minimise the sum of squares of the residuals, ie. determine the minimum of $S$ for $N$ data points where

$$
S=\sum_{i=1}^{N} \varepsilon_{i}^{2}
$$

The sum $S$ is a function of the coefficients $a_{n}^{m}$ and $b_{n}^{m}$ which number $T=(L+1)^{2}$. If the coefficients are ordered and labelled $\left[x_{i}\right]_{i=1,2, \ldots, T}$ and the spherical harmonic functions $\left[s_{i}\right]_{i=1,2, \ldots, T}$, as in Brett (1988), the minimum of $S$ is therefore given by

$$
\frac{\partial S}{\partial x_{i}}=0 \quad \forall i=1,2, \ldots, T .
$$

This forms the basis of the LS method for estimating model parameters. Minimising the square of residuals, called the $L_{2}$ norm, is appropriate when it may be assumed that the errors are random and obey a Gaussian distribution (Draper and Smith, 1981). An alternative method involving minimisation of the magnitude of residuals, called the $L_{1}$ norm, may be applied when the errors are more spread out than the Gaussian distribution. However, the LS method may be adapted for use when Gaussian statistics break down as shown later (Section 6.4.2). Carrying out the differentiation of $S$ with respect to some coefficient $x_{Q}$, where $Q$ is integer and between 1 and $T$ gives

$$
\sum_{i=1}^{N} s_{Q}\left(\theta^{\prime}{ }_{i}, \phi_{i}^{\prime}{ }_{i}\right)\left\{x_{1} s_{1}\left(\theta^{\prime}{ }_{i}, \phi_{i}^{\prime}\right)+\ldots+x_{Q} s_{Q}\left(\theta^{\prime}{ }_{i}, \phi_{i}^{\prime}\right)+\ldots+x_{T} s_{T}\left(\theta^{\prime}{ }_{i}, \phi_{i}^{\prime}\right)\right\}=\sum_{i=1}^{N} s_{Q}\left(\theta^{\prime}{ }_{i}, \phi_{i}^{\prime}\right) X_{i}
$$


The whole system of equations may be written in matrix form

$$
\left(\begin{array}{ccccc}
\sum s_{1} s_{1} & \cdots & \sum s_{1} s_{Q} & \cdots & \sum s_{1} s_{T} \\
\vdots & & \vdots & & \vdots \\
\sum s_{Q} s_{1} & \cdots & \sum s_{Q} s_{Q} & \cdots & \sum s_{Q} s_{T} \\
\vdots & & \vdots & & \vdots \\
\sum s_{T} s_{1} & \cdots & \sum s_{T} s_{Q} & \cdots & \sum s_{T} s_{T}
\end{array}\right)\left(\begin{array}{c}
x_{1} \\
\vdots \\
x_{Q} \\
\vdots \\
x_{T}
\end{array}\right)=\left(\begin{array}{c}
\sum s_{1} X \\
\vdots \\
\sum s_{Q} X \\
\vdots \\
\sum s_{T} X
\end{array}\right)
$$

where the summations extend over the points of the sphere for the observations $X$ and spherical harmonic functions s. Equation 3.40 may be written simply as

$$
A \hat{\mathbf{x}}=\mathbf{b}
$$

where $A$ is the above matrix, $\hat{\mathbf{x}}$ the vector of model coefficient estimates and $\mathbf{b}$ the vector of the product of spherical harmonic functions and observations. The vector $\hat{\mathbf{x}}$ is then given by

$$
\hat{\mathbf{x}}=A^{-1} \mathbf{b}
$$

The matrix $A^{-1}$ is the inverse of $A$. Thus the elements of $\hat{\mathbf{x}}$, the shc's, are determined which give the LS 'best fit' to the observations.

\subsubsection{Separation of Internal and External Parts}

Equation 3.38 may be used as a model in the LS fit of the measurements of $X$ to yield estimates of $a_{n}^{m}, b_{n}^{m} \forall m, n$ represented by $x_{i}$ in Equation 3.40 . In the same manner $a_{n}^{m}, b_{n}^{m}$ may be found from the spatial variation in $Y$ over the sphere. It is required to find the contribution from internal and external sources of potential, i.e. the values of $g_{n}^{m}, h_{n}^{m}, g_{n}^{\prime m}$ and $h_{n}^{\prime m}$ of Equation 3.26. Consider the shc represented by $x_{Q}$ of Equation 3.40 for which $m=\alpha, n=\beta$. Thus from either $X$ or $Y$

$$
\begin{aligned}
& a_{\beta}^{\alpha}=g_{\beta}^{\alpha}+g_{\beta}^{\prime \alpha} \\
& b_{\beta}^{\alpha}=h_{\beta}^{\alpha}+h_{\beta}^{\prime \alpha}
\end{aligned}
$$

where $a_{\beta}^{\alpha}$ and $b_{\beta}^{\alpha}$ have been determined by LS. To separate the internal and external parts more information is needed. Equation 3.29 may be written

$$
Z_{M}=\sum_{n=1}^{L} \sum_{m=0}^{n}\left\{c_{n}^{m} \cos m \phi^{\prime}+d_{n}^{m} \sin m \phi^{\prime}\right\} P_{n}^{m}\left(\cos \theta^{\prime}\right)
$$


where $Z_{M}$ is the $Z$ component derived from the potential. If $m$ and $n$ equal $\alpha$ and $\beta$, as above, the coefficients of Equation 3.43 are

$$
\begin{aligned}
& c_{\beta}^{\alpha}=-(n+1) g_{\beta}^{\alpha}+n g_{\beta}^{\prime \alpha} \\
& d_{\beta}^{\alpha}=-(n+1) h_{\beta}^{\alpha}+n h_{\beta}^{\prime \alpha}
\end{aligned}
$$

The LS method is then applied to the observations of the $Z$ component to find the estimates of $c_{\beta}^{\alpha}$ and $d_{\beta}^{\alpha}$ as in the above section. Two sets of simultaneous equations are then given; Equations 3.41 and 3.44 provide $g_{\beta}^{\alpha}$ and $g_{\beta}^{\prime \alpha}$ while Equations 3.42 and 3.45 may be solved for $h_{\beta}^{\alpha}$ and $h_{\beta}^{\prime \alpha}$. The distinct internal and external sources of potential may be found in this way for every spherical harmonic term of interest. 


\section{Chapter 4}

\section{Data Management}

\subsection{Compilation of Storm Database}

\subsubsection{Data Access}

The source of data for the study was the Compact Disc Read Only Memory (CDROM) produced by World Data Centre - A in Boulder, Colorado, USA. This CD-ROM, containing geomagnetic and other solar-terrestrial data, was prepared as a prototype to investigate the potential of data archiving and accessibility on the medium of compact disc. This version has been assigned the name NGDC01.

To facilitate data retrieval, software was supplied to allow data to be captured and placed in files on the PC connected to the CD-reader. To extract data a series of menus have to be negotiated. The type of data required, hourly values for this study, and the desired time interval must be selected for every chosen observatory. Thus it was necessary for the procedure to be repeated for each observatory of interest and for each time interval defining a storm event. An additional supplied program produced graphical plots of the data on the screen of the PC.

The data preparation and analysis was to be carried out on the University mainframe computer and therefore a rapid means of transferring data, from PC to mainframe, was required. However, the capability for sufficiently fast data transfer was not available at the site of the PC and CD-reader. A different approach to the selection of data had to be implemented.

As selecting data for individual storms directly from NGDC01 and subsequently moving the storm data to the mainframe was not possible it was decided to move the entire hourly database to the mainframe. This was possible as an ethernet connection 
was temporarily available ${ }^{1}$. The complete database of hourly values occupies over 285 Mbytes, therefore the data transfer was only possible after securing sufficient disc space on the mainframe. Thankfully the required space was made available enabling storage.

However, with the data stored on the mainframe the supplied software could not be used to extract portions of data. An alternative program was thus required that would be suitable for the selection of data from many observatories. Code was written to extract data between two dates defining the storm event for all observatories within a specified limit of geomagnetic latitude. It was then possible to efficiently retrieve the required data for a given time interval.

\subsubsection{Data Retrieval}

It was desired to collect together data corresponding to as many suitable storm events as possible. An important consideration is the number and distribution of observatories in operation for the duration of the storm event. It is therefore worthwhile to consider the availability of data over the worldwide observatory network before extracting data for a particular storm. The greater the number of observatories and more even the distribution the better. A means of determining the most profitable periods of time was then necessary.

This information is provided by the Combined International Catalog of Geomagnetic Data (Report UAG-92, 1985) and the catalogue was included on NGDC01. It was, however, found that the data written on to the CD-ROM NGDC01 was not entirely consistent with the catalogue. An indication of the coverage of hourly values could, however, be inferred from the smaller monthly dataset. The monthly values, derived from averaging the hourly values, were used to produce a diagram to indicate hourly data coverage against time and over geomagnetic co-latitude, see Figure 4.1. It is possible that there may be slight discrepancies in the availability of data between the monthly and hourly datasets, though they did always correspond for the data checked. The periods with the greatest data coverage were then chosen using Figure 4.1, the International Geophysical Year (IGY) 1957 to 1958 and the International Quiet Sun Year (IQSY) 1964 to 1965 are to be particularly noted. The years from which storms were selected are listed in Table 4.1.

A catalogue was provided by the British Geological Survey (BGS) that listed the occurrence times of storms from 1874 to 1975 . This was used to establish when to investigate the database for the extraction of storm data for the first two periods in Table 4.1. Data was not extracted without first investigating the morphology of the

\footnotetext{
${ }^{1}$ I am grateful to Steve Law of the Mathematics Department for his help in this matter.
} 
Figure 4.1. Availability of Observatory Data on NGDC01. N.B. years refer to 1900's.

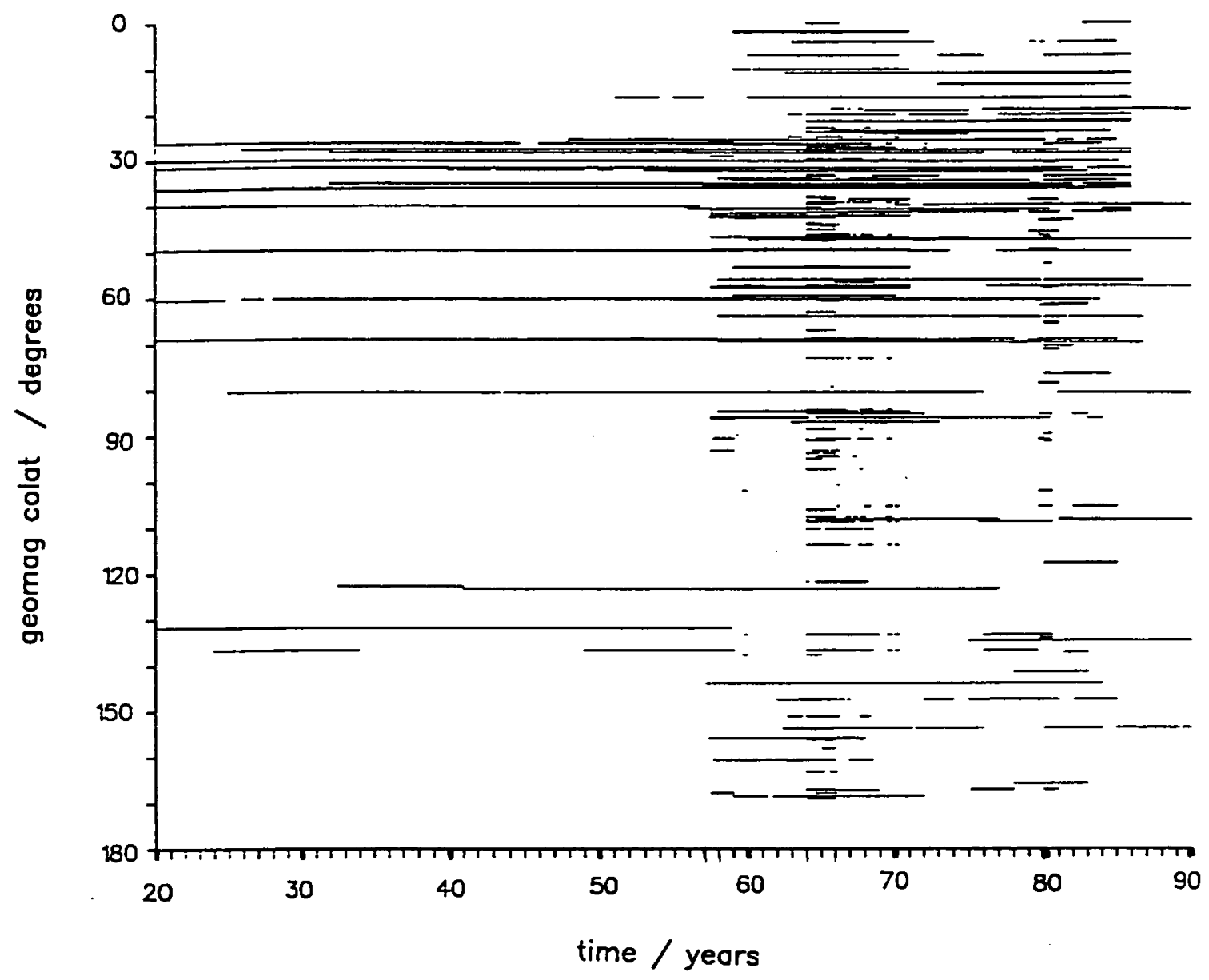

Table 4.1. Periods with greatest density of hourly observations.

\begin{tabular}{|c|c|}
\hline Year at Start & Year at End \\
\hline 1957 & 1958 \\
1964 & 1970 \\
1976 & 1976 \\
1980 & 1982 \\
\hline
\end{tabular}


Dst index (Section 2.1). The CD-ROM NGDC01 and supplied software were used at this stage to plot the index on screen. The $D$ st index is designed to show the temporal variation in the ring current intensity. This was helpful in determining the most clearly defined storms to be selected for analysis. The index was displayed for the whole of the later two periods for which no record of storm onset times existed.

As the time series data were to be transferred to the frequency domain via the Fourier Transform, the intervals were chosen with the following points in mind. Firstly, due to the application of window functions (Section 3.2.2) the main phase of the storm was chosen to be central to the record. Secondly, as far as possible, disturbances near the ends of the interval were avoided.

The storms chosen for this study occur between the dates in Table 4.2. As the same observatories do not function throughout, the storms are each recorded by a different set of observatories. The number of stations that contributed data for each storm are given in the forementioned table, see column labelled 'NS'. Storms marked with an asterisk were analysed in the time domain by Marshall (1980). Tables 4.3 and 4.4 list the complete set of observatories with their co-ordinates, a map showing their locations is given in Figure 4.2. Identification of the European observatories is provided by Figure A.1 in the appendix.

In comparison with the number of observatories used in previous studies (Anderssen et al. , 1970; Marshall, 1980) the data availabilty is rather low. As mentioned above the Catalogue of Geomagnetic Data (Report UAG-92, 1985) did not correlate with the data written on to NGDC01 which omits a significant proportion of the data available at the World Data Centres. This limitation of the CD-ROM seriously reduces knowledge of the spatial structure of the magnetic field. However, due to the adopted approach of analysis (Section 6.3) the number of observatories given by the NGDC01 database should not inhibit the analysis too greatly.

Only observatories between $35^{\circ}$ and $145^{\circ}$ geomagnetic co-latitude were selected for analysis. This was to avoid the more complex source field occuring at high latitudes. The plots of observatory Fourier coefficients against geomagnetic co-latitude (Figures 6.3 and 6.4) confirm the necessity of eliminating observatories near to the geomagnetic poles. A greater spread in the Fourier estimates was observed near to the spatial limits indicating the degradation of the $P_{1}^{0}$ approximation. From this evidence $\pm 55^{\circ}$ would appear to be the maximum geomagnetic latitudes within which observatories should be chosen. More data would result from increasing this limit though the larger dataset would not be amenable to the analysis scheme. 
Table 4.2. The storms chosen for analysis.

\begin{tabular}{|c|c|c|c|}
\hline Storm Number & Start Date & End Date & Number of observatories \\
\hline 1 & $07: 09: 57$ & $20: 09: 57$ & 20 \\
\hline 2 & $27: 01: 58$ & $25: 02: 58$ & 26 \\
\hline 3 & $23: 06: 58$ & 07:07:58 & 26 \\
\hline 4 & 02:07:58 & $17: 07: 58$ & 27 \\
\hline 5 & 28:08:58 & 15:09:58 & 29 \\
\hline $6^{*}$ & $27: 05: 64$ & $24: 06: 64$ & 60 \\
\hline $7^{*}$ & 03:04:65 & 02:05:65 & 67 \\
\hline 8 & 05:06:65 & 03:07:65 & 67 \\
\hline 9 & $06: 03: 66$ & 22:03:66 & 46 \\
\hline 10 & $11: 05: 66$ & $18: 06: 66$ & 39 \\
\hline 11 & 20:08:66 & 25:09:66 & 36 \\
\hline 12 & $07: 12: 66$ & $25: 12: 66$ & 34 \\
\hline 13 & 01:01:67 & $31: 01: 67$ & 32 \\
\hline 14 & $01: 02: 67$ & 02:03:67 & 33 \\
\hline 15 & $16: 05: 67$ & 05:06:67 & 33 \\
\hline 16 & $30: 05: 67$ & $16: 06: 67$ & 33 \\
\hline 17 & $21: 12: 67$ & 21:01:68 & 31 \\
\hline $18^{*}$ & $26: 01: 69$ & $10: 02: 69$ & 29 \\
\hline $19^{*}$ & $06: 02: 69$ & $18: 02: 69$ & 28 \\
\hline 20 & 12:03:69 & 11:04:69 & 29 \\
\hline 21 & 01:05:69 & $31: 05: 69$ & 29 \\
\hline 22 & $16: 07: 69$ & 12:08:69 & 27 \\
\hline 23 & $17: 09: 69$ & $15: 10: 69$ & 32 \\
\hline $24^{*}$ & 27:02:70 & 18:03:70 & 29 \\
\hline 25 & 10:04:70 & $10: 05: 70$ & 27 \\
\hline 26 & $16: 05: 70$ & $11: 06: 70$ & 28 \\
\hline 27 & 01:07:70 & 20:07:70 & 28 \\
\hline 28 & $14: 07: 70$ & 07:08:70 & 28 \\
\hline $29^{*}$ & 06:08:70 & 30:08:70 & 28 \\
\hline 30 & 28:10:70 & $17: 11: 70$ & 28 \\
\hline 31 & $01: 12: 70$ & $31: 12: 70$ & 28 \\
\hline 32 & 01:01:76 & $25: 01: 76$ & 19 \\
\hline 33 & 11:03:76 & 15:04:76 & 18 \\
\hline 34 & $16: 04: 76$ & 18:05:76 & 18 \\
\hline 35 & 03:01:80 & $26: 01: 80$ & 42 \\
\hline 36 & 02:02:80 & 03:03:80 & 46 \\
\hline 37 & 07:12:80 & 06:01:81 & 25 \\
\hline 38 & $27: 01: 81$ & $19: 02: 81$ & 18 \\
\hline 39 & $20: 02: 81$ & 23:03:81 & 18 \\
\hline 40 & 01:04:81 & $30: 04: 81$ & 18 \\
\hline 41 & $11: 07: 81$ & 08:08:81 & 17 \\
\hline 42 & 29:06:82 & $29: 07: 82$ & 16 \\
\hline 43 & $23: 08: 82$ & 21:09:82 & 17 \\
\hline 44 & $14: 11: 82$ & $06: 12: 82$ & 17 \\
\hline
\end{tabular}


Table 4.3. The Observatories used in the Storm Analysis.

\begin{tabular}{|c|c|c|c|c|c|c|c|}
\hline \multirow[t]{2}{*}{ Location } & \multirow[t]{2}{*}{ Code } & \multirow[t]{2}{*}{ NS } & \multicolumn{2}{|c|}{ Geographic Co-ordinates } & \multicolumn{2}{|c|}{ Geomagnetic Co-ordinateg } & \multirow{2}{*}{$\begin{array}{r}\text { Rotation } \\
\psi\left({ }^{\circ}\right)\end{array}$} \\
\hline & & & $\begin{array}{r}\text { Co-latitude } \\
\theta\left({ }^{\circ}\right) \\
\end{array}$ & $\begin{array}{r}\text { East-longitude } \\
\phi\left({ }^{\circ}\right)\end{array}$ & $\begin{array}{r}\text { Co-latitude } \\
\theta^{\prime}\left({ }^{\circ}\right)\end{array}$ & $\begin{array}{r}\text { East-Iongitude } \\
\phi^{\prime}\left({ }^{\circ}\right)\end{array}$ & \\
\hline alma ata & asa & 11 & 46.750 & 76.917 & 58.554 & 150.713 & -7.695 \\
\hline addis ababa & ase & 30 & 80.970 & 38.765 & 84.622 & 109.148 & -10.994 \\
\hline alibag. & abg & 31 & 71.362 & 72.872 & 80.528 & 143.621 & -7.169 \\
\hline argentine islands & ais & 44 & 155.245 & 295.742 & 143.773 & 3.358 & -1.598 \\
\hline almeris & alm & 4 & 53.147 & 357.540 & 49.365 & 75.298 & -13.945 \\
\hline martin de vivies & ams & 3 & 127.833 & 77.567 & 137.093 & 140.271 & -9.285 \\
\hline annamalainagar & ann & 3 & 78.633 & 79.683 & 88.501 & 149.353 & -5.950 \\
\hline apia & api & 3 & 103.807 & 188.225 & 106.062 & 260.244 & 11.673 \\
\hline 1 aquila & aqu & 36 & 47.617 & 13.317 & 47.138 & 92.942 & -15.638 \\
\hline vannovskays & ash & 18 & 52.050 & 58.108 & 59.484 & 133.115 & -10.635 \\
\hline belsk & bel & 8 & 38.163 & 20.792 & 39.564 & 104.043 & -18.241 \\
\hline beijing & $\mathbf{b j i}$ & 3 & 49.960 & 116.175 & 61.419 & 184.510 & 1.173 \\
\hline beloit & blt & 1 & 50.523 & 261.867 & 40.762 & 324.863 & 8.549 \\
\hline bangui & bng & 19 & 85.563 & 18.565 & 85.167 & 88.500 & -11.531 \\
\hline boulder & bou & 27 & 49.862 & 254.762 & 41.008 & 316.469 & 10.347 \\
\hline burlington & brt & 3 & 50.617 & 257.733 & 41.357 & 320.084 & 9.527 \\
\hline canberra & $\operatorname{can}$ & 2 & 125.315 & 149.363 & 133.949 & 224.703 & 9.896 \\
\hline carrollton & $\operatorname{cax}$ & 2 & 50.633 & 266.467 & 40.380 & 330.298 & 7.341 \\
\hline chambon-la-foret & clf & 1 & 41.977 & 2.260 & 39.527 & 84.378 & -17.256 \\
\hline changchun & cnh & 3 & 46.173 & 125.299 & 57.370 & 192.215 & $\mathbf{3 . 3 5 2}$ \\
\hline coimbra & coi & 3 & 49.793 & 351.577 & 44.977 & 70.242 & -14.222 \\
\hline port alfred & czt & 8 & 136.433 & 51.867 & 141.308 & 108.856 & -15.887 \\
\hline dallas & dal & 23 & 57.015 & 263.248 & 47.040 & 327.743 & 7.288 \\
\hline dourbes & dou & 3 & 39.903 & 4.595 & 38.015 & 87.711 & -18.092 \\
\hline ebro & ebr & 5 & 49.180 & 0.493 & 46.099 & 79.649 & -15.020 \\
\hline fanning & fan & 5 & 86.095 & 200.610 & 86.251 & 268.838 & 11.525 \\
\hline fredericksburg & frd & 36 & 51.795 & 282.627 & 40.443 & 349.840 & 2.565 \\
\hline fuquene & fuq & 3 & 84.530 & 286.263 & 73.071 & 355.071 & 0.986 \\
\hline furstenfeldbruck & fur & 11 & 41.835 & 11.277 & 41.187 & 93.322 & -17.362 \\
\hline gnangaro & gna & 7 & 121.783 & 115.950 & 133.233 & 185.778 & 1.353 \\
\hline guam & gua & 34 & 76.417 & 144.870 & 86.030 & 212.890 & 6.395 \\
\hline guangzhou & gzh & 3 & 66.907 & 113.343 & 78.398 & 182.200 & 0.477 \\
\hline hartland & had & 41 & 39.005 & 355.517 & 35.365 & 79.001 & -18.116 \\
\hline hermanus & her & 34 & 124.425 & 19.225 & 123.290 & 80.515 & -13.792 \\
\hline honolulu & hon & 35 & $\mathbf{6 8 . 6 8 0}$ & 201.937 & 68.942 & 266.464 & 12.334 \\
\hline hurbanovo & hrb & 6 & 42.127 & 18.190 & 42.834 & 99.793 & -17.031 \\
\hline huancayo & hus & 8 & 102.045 & 284.660 & 90.613 & 353.800 & 1.262 \\
\hline irkutak & irt & 33 & $\mathbf{3 7 . 7 3 3}$ & 104.267 & 49.169 & 174.559 & -1.770 \\
\hline jarvis island & jrv & 4 & 90.383 & 199.967 & 90.581 & 269.064 & 11.499 \\
\hline kakioks & kak & 42 & 53.770 & 140.190 & 63.975 & 205.964 & 6.212 \\
\hline bereznyali & kgd & 2 & 40.183 & 73.083 & 48.672 & 148.660 & -9.248 \\
\hline kiev & kiv & 3 & 39.283 & $\mathbf{3 0 . 3 0 0}$ & 42.431 & 112.168 & -16.954 \\
\hline kanoya & kny & 42 & 58.580 & 130.882 & 69.461 & 198.054 & 4.152 \\
\hline kanozan & $\mathbf{k n z}$ & 3 & 54.747 & 139.860 & 64.966 & 205.874 & 6.116 \\
\hline kodaikanal & kod & 2 & 79.770 & 77.463 & 89.399 & 147.063 & -6.324 \\
\hline koror & kor & 6 & 82.667 & 134.500 & 83.225 & 203.336 & 4.567 \\
\hline kazan & ken & 3 & 34.217 & 49.133 & 40.760 & 130.577 & -15.622 \\
\hline leadville & ldv & 4 & 50.717 & 253.717 & 41.993 & 315.508 & 10.399 \\
\hline logrono & $\lg x$ & 3 & 47.542 & 357.495 & 43.932 & 77.196 & -15.278 \\
\hline maputo & lmm & 10 & 115.917 & 32.583 & 117.665 & 95.821 & -12.739 \\
\hline lunping & $\operatorname{lnp}$ & 10 & 65.000 & 121.167 & 76.333 & 189.476 & 2.076 \\
\hline la quiaca & lqa & 1 & 112.110 & 294.417 & 100.629 & 3.221 & -0.693 \\
\hline luanda belas & lua & 3 & 98.917 & 13.167 & 97.184 & 80.553 & -11.483 \\
\hline lvov & lvv & 31 & 40.100 & 23.750 & 41.978 & 105.860 & -17.322 \\
\hline Iwiro & lwi & 3 & 92.250 & 28.800 & 93.756 & 97.200 & -11.417 \\
\hline lanzhou & lah & 3 & 53.913 & 103.845 & 65.334 & 173.641 & -1.566 \\
\hline
\end{tabular}


Table 4.4. The Observatories used in the Storm Analysis (cont.).

\begin{tabular}{|c|c|c|c|c|c|c|c|}
\hline \multirow[t]{2}{*}{ Location } & \multirow[t]{2}{*}{ Code } & \multirow[t]{2}{*}{ NS } & \multicolumn{2}{|c|}{ Geographic Co-ordinates } & \multicolumn{2}{|c|}{ Geomagnetic Co-ordinates } & \multirow{2}{*}{$\begin{array}{r}\text { Rotation } \\
\psi\left({ }^{\circ}\right)\end{array}$} \\
\hline & & & $\begin{array}{r}\text { Co-latitude } \\
\theta\left({ }^{\circ}\right)\end{array}$ & $\begin{array}{r}\text { East-longitude } \\
\phi\left({ }^{\circ}\right)\end{array}$ & $\begin{array}{r}\text { Co-latitude } \\
\theta^{\prime}\left({ }^{\circ}\right)\end{array}$ & $\begin{array}{r}\text { East-longitude } \\
\phi^{\prime}\left({ }^{\circ}\right)\end{array}$ & \\
\hline m bour & mbo & 10 & 75.608 & 343.042 & 68.756 & 55.025 & -9.710 \\
\hline moca & mfp & 8 & 86.657 & 8.660 & 84.279 & 78.555 & -11.288 \\
\hline mizusawa & $\operatorname{miz}$ & 10 & 50.990 & 141.080 & 61.134 & 206.405 & 6.552 \\
\hline misallat & mlt & 3 & 60.485 & 30.892 & 63.066 & 105.932 & -12.727 \\
\hline memambetsu & mmb & 43 & 46.093 & 144.193 & 55.988 & 208.414 & 7.566 \\
\hline pleshenitzi & mnk & 6 & 35.500 & 27.883 & 38.381 & 111.793 & -18.590 \\
\hline muntinlupa & mut & 25 & 75.625 & 121.015 & 86.956 & 189.712 & 1.990 \\
\hline nairobi & nai & 3 & 91.327 & 36.815 & 94.417 & 105.257 & -11.092 \\
\hline niemegk & ngk & 3 & 37.928 & 12.675 & $\mathbf{3 7 . 7 5 0}$ & 96.570 & -18.797 \\
\hline novokazalingk & nok & 2 & 44.200 & 62.100 & 52.327 & 138.415 & -10.942 \\
\hline nampula & nmp & 1 & 105.087 & 39.253 & 108.382 & 104.928 & -11.509 \\
\hline klyuchi & nvs & 2 & 34.967 & 82.900 & 45.393 & 157.718 & -7.579 \\
\hline odessa & ode & 32 & 43.567 & 30.766 & 46.629 & 110.874 & -15.681 \\
\hline paramaribo & pab & 7 & 84.190 & 304.778 & 73.032 & 14.343 & -2.845 \\
\hline panagyurishte & pag & 3 & 47.485 & 24.177 & 49.151 & 103.358 & -15.258 \\
\hline price & pcu & 4 & 50.400 & 248.167 & 42.346 & 310.279 & 11.385 \\
\hline pilar & pil & 10 & 121.667 & 296.117 & 110.208 & 4.640 & -1.086 \\
\hline port moresby & pmg & 8 & 99.408 & 147.152 & 108.602 & 217.885 & 7.129 \\
\hline podkamennaya tungus & pod & 2 & 28.400 & 80.000 & 39.334 & 164.400 & -6.472 \\
\hline pamatai & ppt & 5 & 107.568 & 210.425 & 105.346 & 282.770 & 11.768 \\
\hline pruhonice & pru & 1 & 40.010 & 14.547 & 40.098 & 97.305 & -17.913 \\
\hline roburent & rob & 2 & 45.703 & 7.888 & 44.215 & 88.367 & -16.167 \\
\hline sabhowala & sab & 7 & 59.637 & 77.798 & 69.428 & 149.691 & -6.696 \\
\hline south georgia & sge & 10 & 144.516 & 323.983 & 134.496 & 26.296 & -8.752 \\
\hline $\operatorname{san}$ juan & sjg & 43 & 71.618 & 293.882 & 60.134 & 3.154 & -0.662 \\
\hline sheshan & $88 h$ & 7 & 58.903 & 121.187 & 70.239 & 189.260 & 2.147 \\
\hline simosato & 880 & 3 & 56.425 & 135.940 & 66.964 & 202.443 & 5.241 \\
\hline surlari & sua & 3 & 45.320 & 26.253 & 47.464 & 106.071 & -15.630 \\
\hline sverdlovsk & svd & 33 & 33.173 & 60.638 & 41.355 & 140.377 & -13.437 \\
\hline swider & swi & 3 & $\mathbf{3 7 . 8 8 5}$ & 21.253 & 39.389 & 104.605 & -18.311 \\
\hline tahiti & tah & 1 & 107.555 & 210.388 & 105.341 & 282.732 & 11.769 \\
\hline tananarive & $\tan$ & 9 & 108.916 & 47.550 & 113.702 & 112.454 & -11.231 \\
\hline tehran & teh & 2 & 54.263 & 51.382 & 60.627 & 126.531 & -11.383 \\
\hline teoloyucan & teo & 2 & 70.253 & 260.818 & 60.443 & 327.045 & 6.617 \\
\hline tbilisi & tfs & 26 & 48.282 & 44.797 & 53.697 & 122.063 & -13.083 \\
\hline tihany & thy & 1 & 43.100 & 17.893 & 43.707 & 99.100 & -16.745 \\
\hline tashlrent & tkt & 32 & 48.667 & 69.300 & 57.629 & 143.742 & -9.034 \\
\hline tangerang & $\operatorname{tng}$ & 3 & 96.167 & 106.633 & 107.632 & 175.444 & -0.913 \\
\hline toledo & tol & 5 & 50.117 & 355.953 & 46.123 & 74.675 & -14.511 \\
\hline toolangi & too & 3 & 127.533 & 145.467 & 136.663 & 220.839 & 9.463 \\
\hline trivandrum & trd & $\mathbf{5}$ & 81.484 & 77.000 & 91.051 & 146.419 & -6.402 \\
\hline trelew & trw & 10 & 133.248 & 294.685 & 121.768 & 3.157 & -0.864 \\
\hline tsumeb & tsu & 27 & 109.217 & 17.700 & 108.162 & 82.812 & -12.091 \\
\hline tatuoca & ttb & 2 & 91.200 & 311.480 & 80.434 & 20.787 & -4.058 \\
\hline tucson & tuc & 40 & 57.753 & 249.167 & 49.572 & 312.181 & 10.060 \\
\hline victoria & vic & 39 & 41.483 & 236.583 & 35.810 & 292.971 & 16.088 \\
\hline gornotayezhnaya & vla & 30 & 46.317 & 132.167 & 57.156 & 198.108 & 4.915 \\
\hline vassouras & v8s & 2 & 112.400 & 316.350 & 101.937 & 23.865 & -5.005 \\
\hline watheroo & wat & 5 & 120.318 & 115.877 & 131.770 & 185.647 & 1.302 \\
\hline wuhan & whn & 3 & 59.472 & 114.559 & 70.952 & 183.243 & 0.750 \\
\hline wien-kobenzl & wik & 3 & 41.735 & 16.318 & 42.091 & 98.193 & -17.244 \\
\hline witteveen & wit & 44 & 37.187 & 6.668 & 35.855 & 91.195 & -19.255 \\
\hline wingst & wng & 3 & 36.257 & 9.073 & 35.457 & 94.052 & -19.649 \\
\hline yakutsk & yak & 6 & 27.983 & 129.717 & 39.035 & 193.832 & 5.830 \\
\hline yuzhno sakhalinsk & y88 & 4 & 43.050 & 142.717 & 53.107 & 206.662 & 7.531 \\
\hline
\end{tabular}


Figure 4.2. Location Map of the Observatories. The solid symbols refer to the observatories used in the assessment of the daily variation removal technique (Section 4.2.3).

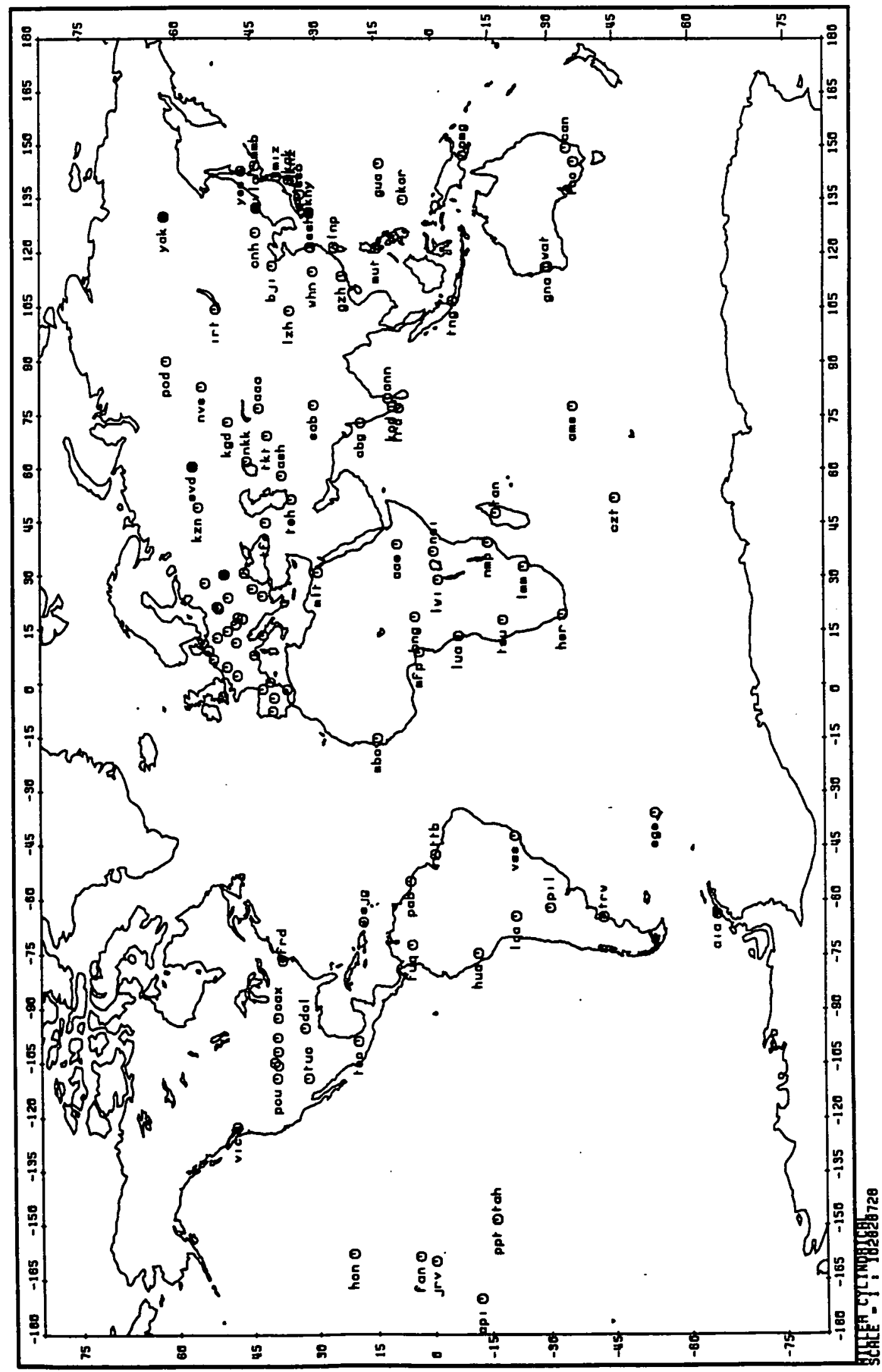




\subsection{Preliminary Processing}

\subsubsection{Data Scrutiny}

It became evident that approximately $5 \%$ of the selected records were contaminated by spikes or changes in the baseline level. Whichever way the storm data is processed, either in the time or frequency domain, eventually the response is required as a function of frequency. Therefore the time series data should be 'cleaned' to remove such features which would severely corrupt the efforts of Fourier analysis.

Due to the amount of data, involving over $1300 X$ and $Y$ component records, an automatic method for correcting errors would be advantageous. Programmable means for recognising spikes and baseline changes were considered. Stepping through the records calculating first differences, and correlation with a delta function to indicate spikes and a step function to reveal baseline changes, may have been used if it were not for the disturbed nature of the time series. It was not found possible to devise a test to distinguish between the abrupt features, such as $S S C$, with a physical significance and the erroneous spikes or shifts in baseline. It was therefore decided to plot the entire set of storm records and inspect them visually. Errors could then be spotted and a method of correction applied.

It was still necessary to have a rule for recognising spikes and baseline changes, and to devise a method for dealing with them. Spikes were taken as being anomalous, short lived deviations having no correlation with the other two magnetic components or with variations at other observatories. They were removed from the time series by simple linear interpolation between the values at either end of the spike. A more persistent 'spike like' feature, of over 3 hours duration, was replaced with 9999's, denoting missing data, to be dealt with later (Section 4.2.4).

A change in the magnetic field not followed by a subsequent change of similar magnitude in the opposite sense, such that the mean value either side of the discontinuity differs is referred to as a baseline change. To remove errors of this type the values to one side of the baseline change were shifted by the appropriate amount to eliminate the discontinuity. In the case of records which were undisturbed during the initial and final day the magnitude of the change was determined by calculating the difference in these daily means. Otherwise the size of the correction was determined by visually inspecting the record. 


\subsubsection{Conversion of Magnetograms to the Geomagnetic Dipole Frame of Reference}

The spatial analysis (Chapter 6 ) requires the magnetic field to be defined in terms of north $(X)$, east $(Y)$ and vertical $(Z)$ components measured with respect to the geomagnetic dipole. It was therefore necessary to transform the data to this format. The majority of data stored on NGDC01 is defined as declination $(D)$, horizontal $(H)$ and vertical components, in terms of geographic co-ordinates. A small portion was recorded as $X, Y$ and $Z$ with regard to the geographic poles. The first task was then to convert to a local Cartesian co-ordinate system for the records not conforming to $X, Y, Z$. The relationships given in Equation 4.1 were used to achieve this requirement.

$$
X=H \cos D \quad Y=H \sin D
$$

Once this had been done a rotational transformation was then necessary to convert the $X$ and $Y$ components from geographic to geomagnetic co-ordinates. This spherical trigonometric transformation was carried out as described below.

To define a point on the surface of the Earth co-latitude or latitude, measured with respect to the geographic axis, and east-longitude, measured from the Greenwich meridian, are used in most circumstances. For the purpose of this study it was necessary to change the frame of reference. Consider the spherical triangle BNP (Figure 4.3), where $B$ is the northern end of the geomagnetic dipole axis, $N$ is the north geographic pole and $\mathrm{P}$ the position of some observatory. Although not shown $\mathrm{O}$ is taken as the position of the Earth's centre and $A$ the southern end of the geomagnetic dipole. It was required to define co-latitude and east-longitude of $P$ relative to the axis $O B$ and meridian BNA. Referring to the angles of Figure 4.3, the cosine formula of spherical trigonometry enabled calculation of the the geomagnetic co-latitude $\theta^{\prime}$ (Equation 4.2).

$$
\cos \theta^{\prime}=\cos \theta \cos \theta_{0}+\sin \theta \sin \theta_{0} \cos \left(\phi-\phi_{0}\right)
$$

Once $\theta^{\prime}$ had been found the cosine formula was applied again to give the geomagnetic east-longitude $\left(\phi^{\prime}\right)$ (Equation 4.3). If the geographic east-longitude of $P(\phi)$ was greater than $\phi_{o}-180^{\circ}$ and less than $\phi_{o}$, the angle $\phi^{\prime}$ obtained from Equation 4.3 was replaced by $360^{\circ}-\phi^{\prime}$. This was done to reflect the solution into the correct quadrant.

$$
\cos \phi^{\prime}=\frac{\cos \theta_{0} \cos \theta^{\prime}-\cos \theta}{\sin \theta_{0} \sin \theta^{\prime}}
$$

For the components $X$ and $Y$ to be aligned with respect to the dipole axis the angle BPN had to be determined, the sine formula gives Equation 4.4.

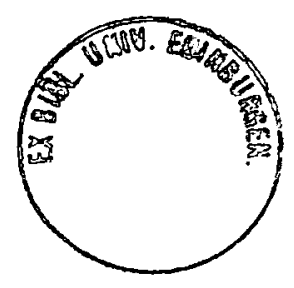




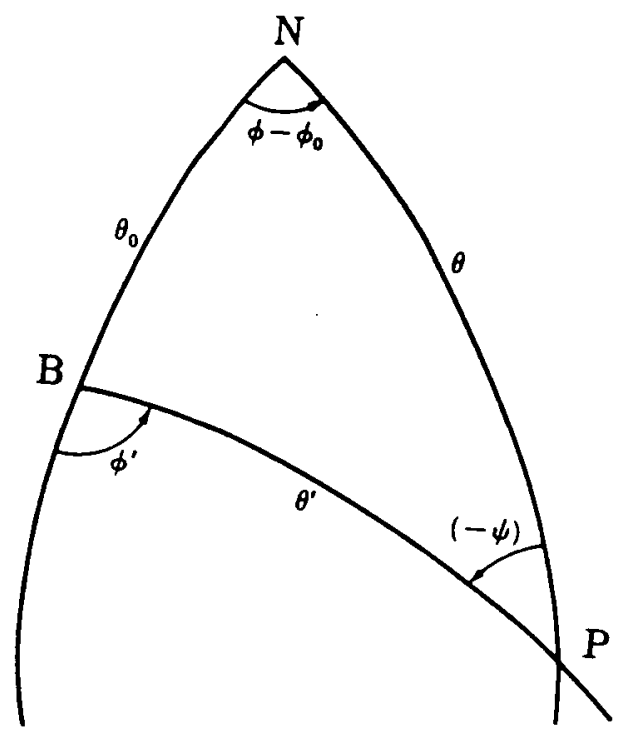

Figure 4.3. The geometry in computing geomagnetic co-ordinates and angle of rotation at an observatory $\mathrm{P}, \mathrm{N}$ is the geographic north pole and $\mathrm{B}$ the northern end of the dipole axis. After Akasofu and Chapman (1972). 
Table 4.5. North Geomagnetic Pole Positions from fifth generation IGRF. After Langel et al. (1988).

\begin{tabular}{|c|c|c|}
\hline Year & Co-latitude $\left(^{\circ}\right)$ & East-Longitude $\left(^{\circ}\right)$ \\
\hline 1955 & 11.54 & 290.84 \\
1965 & 11.47 & 290.15 \\
1975 & 11.31 & 289.53 \\
1985 & 11.02 & 289.10 \\
\hline
\end{tabular}

$$
\sin (-\psi)=\frac{\sin \theta_{o} \sin \phi^{\prime}}{\sin \theta}
$$

The geomagnetic north pole $B$ was taken to be located at $\left(11.5^{\circ}, 291.0^{\circ}\right)$ in geographic co-ordinates, as used in previous studies (eg. Marshall, 1980; Roberts, 1984). However, the fifth generation IGRF models (Table 4.5) show a small continual shift in the position of the north geomagnetic pole during the time span of the selected storms. Therefore the most accurate transformation from geographic to geomagnetic coordinates would have been obtained by considering the position of the geomagnetic north pole for the year the storm occurred. However, this should not have had any significant detrimental effect due to the small change and the choice of $B$ which falls within the range of geomagnetic north pole variation. Figure 4.4 shows the positions of the observatories plotted with respect to the geographic co-ordinate and geomagnetic co-ordinate systems.

\subsubsection{Removal of the Daily Variation}

It was mentioned in Section 2.1 that magnetograms show a periodic variation of fundamental frequency $1 \mathrm{cpd}$ which is due to the Earth's rotation about its axis. The removal of this signal is critical for the analysis of storm data in the time domain (e.g. Marshall, 1980). For a frequency domain study its removal is not absolutely necessary when it is not required to yield information at $1 \mathrm{cpd}$ or higher frequencies. However, the method for generating values when there are gaps in the observations (Section 4.2.4), assumes that the magnetic field variations change only in respect to Universal Time. It is therefore necessary to reduce the Local Time dependent daily variation which would hinder the technique of Section 4.2.4. Only reduction, as opposed to complete elimination, is possible as the variation is not perfectly periodic but has a partially disturbed nature, the $D S$ of Section 2.1. The unpredictable part of the daily variation cannot be removed by the methods described below, which determine the average periodic signal, approximating $S D$, throughout the time series. 
Figure 4.4. The Observatory Locations with respect to a) Geographic Co-ordinates and b) Geomagnetic Co-ordinates.
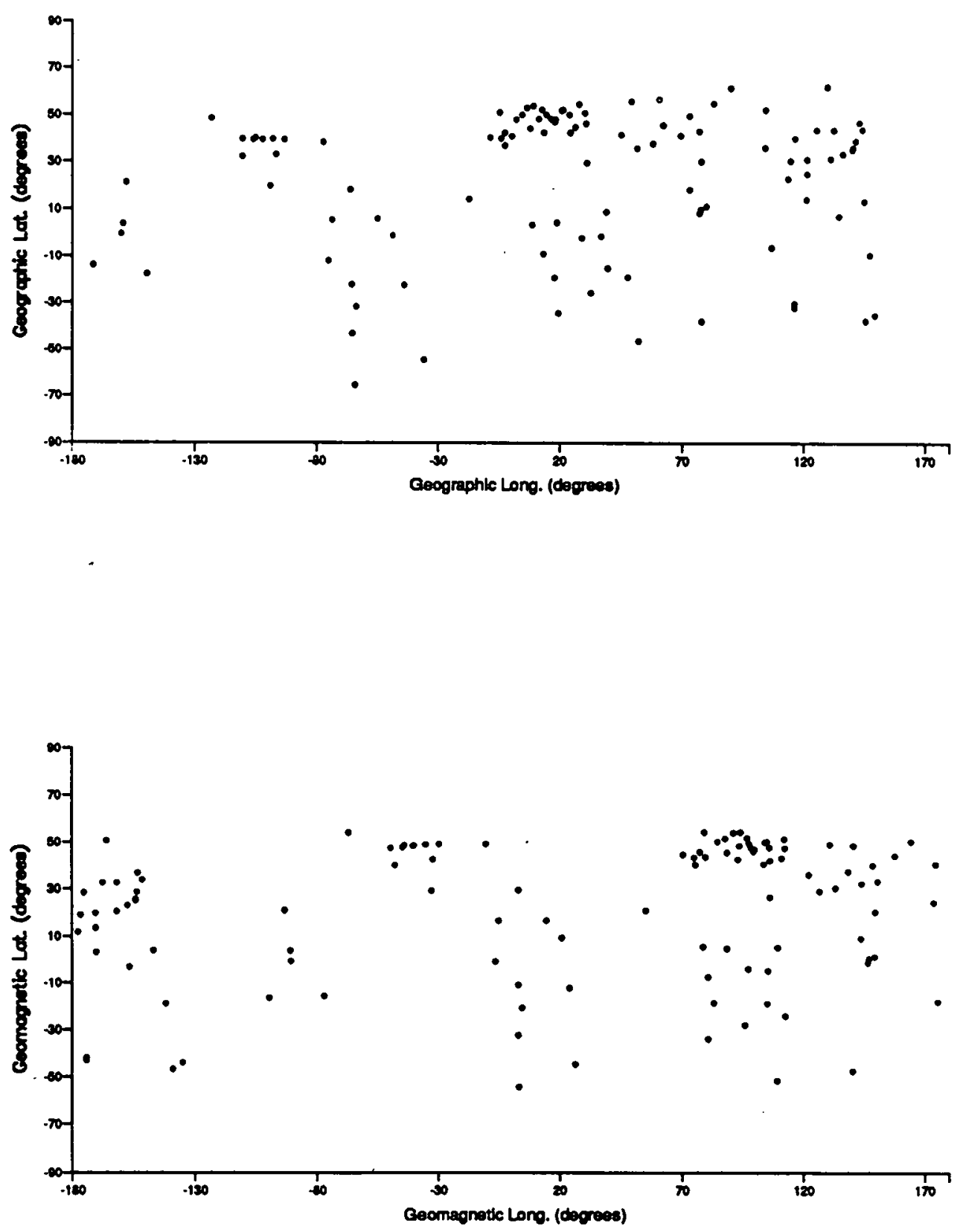
The manner in which this problem has been tackled previously has been to represent the daily variation by a finite Fourier series and to subsequently remove it from the original record (Anderssen et al. , 1970; Marshall, 1980). This cannot be carried out for the entire time series, however, due to the presence of the storm, it may only be adopted during undisturbed stretches of data. This means that the diurnal variation taken away from the whole record may not be the most appropriate estimate. It also requires some section of the series to be chosen for application of the finite Fourier representation, this being a difficult task to carry out automatically on numerous records of varying characteristics.

To make an estimate of the daily variation over the whole record including the storm a frequency domain technique was used. The magnetic time series were Fourier transformed to the frequency domain where an estimate of the daily variation was made. The time series of the estimated diurnal signal was then composed and subtracted from the original time series. A storm record with a much reduced 24 hour component was then produced.

This operation was, however, complicated by the time structure of the storm records. Figure 5.1 reveals the form of the original real and imaginary parts of the Fourier coefficients for a typical storm. The tendency for the coefficients to oscillate positive and negative was noted. The cause of this phenomenon was due to the time of onset of the storm in the central part of the time series. The displacement of energy with respect to the origin of the record introduces a phase angle which shifts each consecutive Fourier coefficient. This phase is near to $180^{\circ}$ so that expressed in com. plex notation positive and negative values occur alternately. Further discussion on this aspect of Fourier analysis is found in Section 5.4.

The spectrum Figure 4.5 shows the presence of extremely high power at the fundamental and harmonics of the daily variation ${ }^{2}$. It was desired to reduce these Fourier coefficients to a level more in keeping with the overall trend with frequency. To achieve this the mean of the four or eight nearest coefficients, depending on record length, was taken as a replacement for the original value. However, the switching sign of the real and imaginary parts would inhibit calculation of a mean value. Alternatively representation in terms of amplitude and phase could be used. Reduction of power is then enabled by replacing the original amplitude, at the specific frequencies, with the mean of its neighbours. The phase was left unaltered as there is no reasoning to argue that it should be changed in a particular manner. A similar philosophy, the Rayleigh method, is adopted in the robust estimation of transfer functions (Chave et al. , 1987). An

\footnotetext{
${ }^{2}$ The 'original' power spectrum is used for what is sometimes called the 'raw' spectrum.
} 
Figure 4.5. Original Power Spectrum for Storm 2, $X$ Component at Addis Ababa (aae).

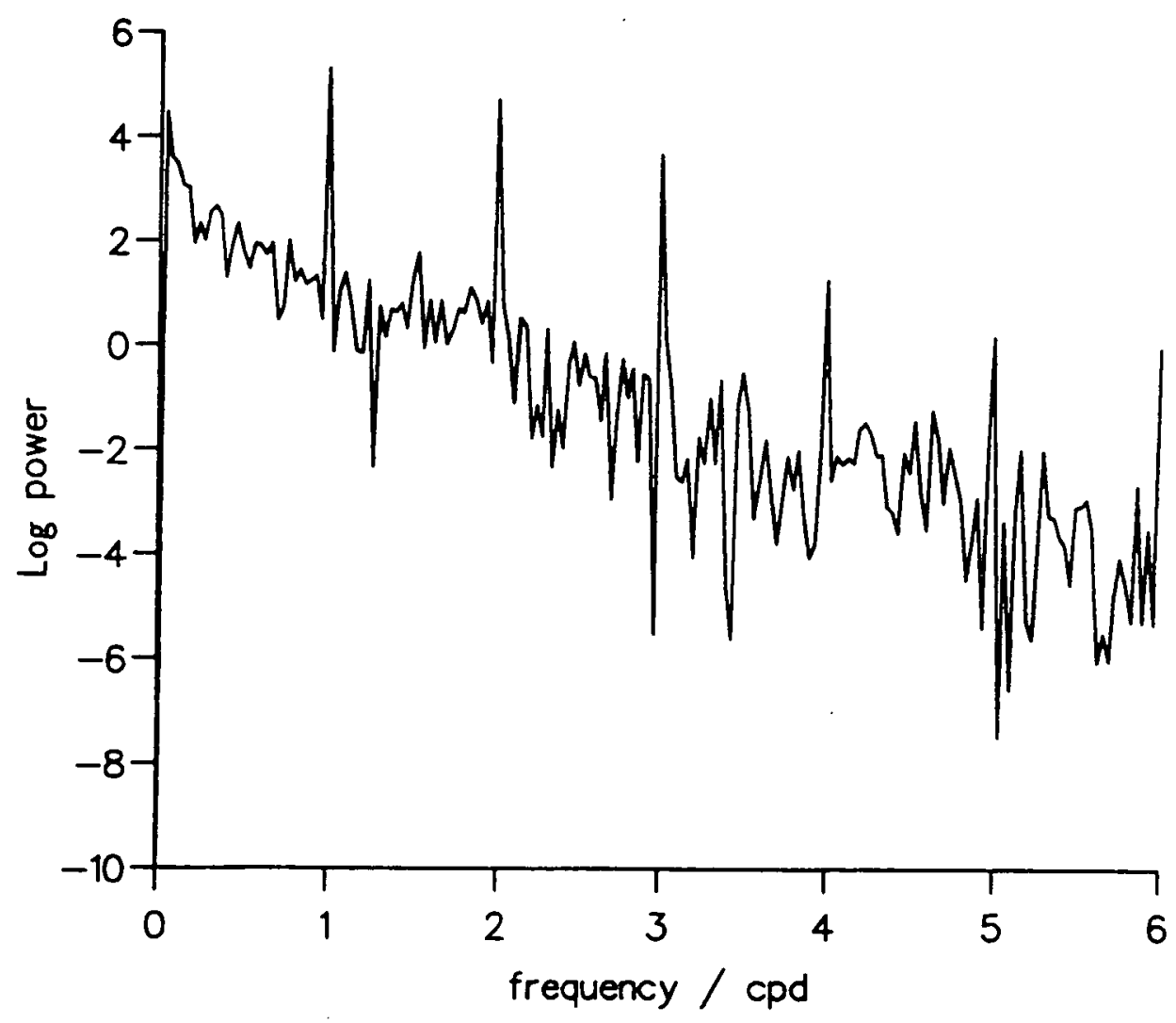


example of the effect this process has is shown in Figures 4.6 and 4.7.

The daily variation was removed to enable the generation of data where values were missing. Estimation and subtraction of the diurnal signal was, however, applied to all observatory records to be consistent in the treatment of data and to enable any observatory to be used in the method of Section 4.2.4.

To provide quantative evidence that removing the daily variation reduced the Local Time dependent signal the correlation coefficient was calculated between a selection of observatories. Table 4.6 gives the correlation for observatories chiefly displaced in eastlongitude $\phi^{\prime}$ while three observatories of varying co-latitude $\theta^{\prime}$ are shown in Table 4.7. These Tables give the correlation before and after application of the described daily variation removal technique. The data were taken from the 696 hour long records of Storm 6, see Table 4.2, and are plotted in Figures 4.6 and 4.7. The locations of the five observatories is indicated by the solid symbols on map of Figure 4.2.

Assessment of the removal technique with respect to dependence on $\phi^{\prime}$ revealed that in all these cases the correlation in the $X$ component increased after its application. The most dramatic increase was between KIV and YAK the most distant pair. This indicates that the Local Time, or $\phi^{\prime}$, dependant signal has decreased and the records are thus more suitable for use in the interpolation method of Section 4.2.4. For the $Y$ component the correlation does not change by very much except in the case between KIV and SVD where the coefficient decreased considerably. A decrease is observed because the stations are quite close together, separated by $28^{\circ}$ in geomagnetic eastlongitude, and therefore the diurnal signal is only out of phase by about two hours. In comparison to the diurnal signal the storm is an insignificant and not well correlated feature of the $Y$ component and thus the correlation coefficient is smaller without the daily variation. It is not required to include the $Y$ component in the analysis of a zonal field, ideally a storm should not be manifest at all in the $Y$ trace, so the correlation in $Y$ has no bearing on the analysis. Consideration of the trend, before and after, in the correlation coefficient for $Z$ shows that in all three cases an increase has occurred. If a gap had occurred in one of the $Z$ component records the other observatory records would thus be more suited to the procedure in the following section (4.2.4).

Although supresssion of the Local Time signal was the objective, the diurnal removal technique was applied to the whole observatory dataset and therefore its affect over colatitude $\theta^{\prime}$ was also considered (Table 4.7). The $X$ component records were more highly correlated after the removal technique. This is so because although the daily variation is in-phase its amplitude and form vary with geomagnetic co-latitude. Therefore the storm dominant $X$ component is correlated to a greater extent without the diurnal signal. The correlation between the $Y$ component records and between the $Z$ component records 
Figure 4.6. Magnetograms before (left) and after (right) removal of the daily variation. Observatories displaced mainly in geomagnetic east-longitude.

$x$ Component

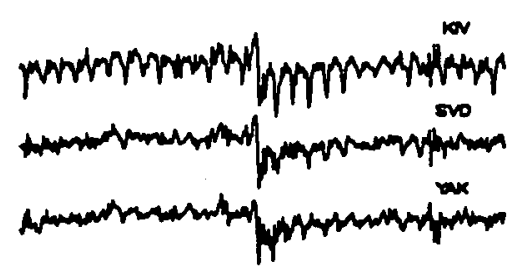

$Y$ Component

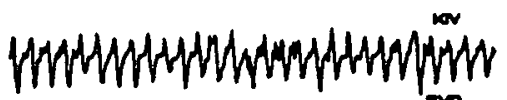

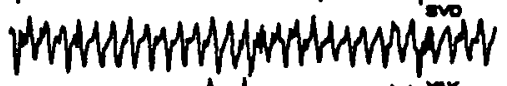
thw

2 Component

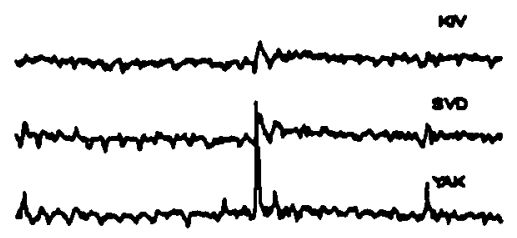

$x$ component
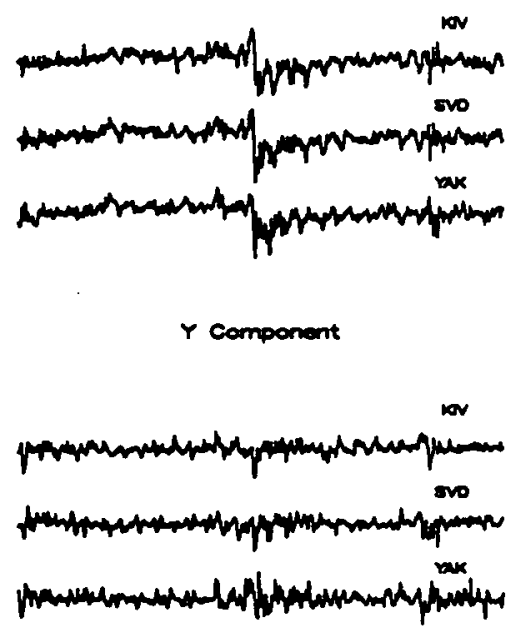

$z$ Component

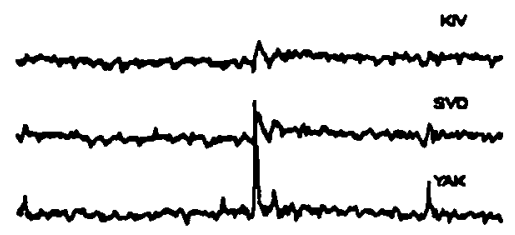


Figure 4.7. Magnetograms before (left) and after (right) removal of the daily variation. Observatories displaced mainly in geomagnetic co-latitude.

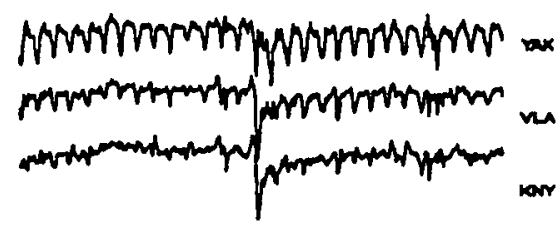

$Y$ Component

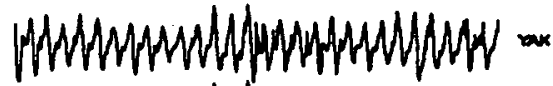

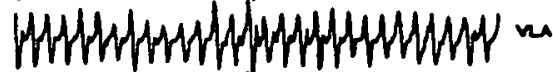

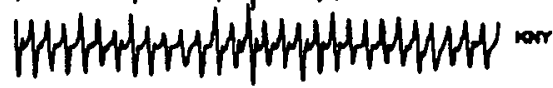

2 component

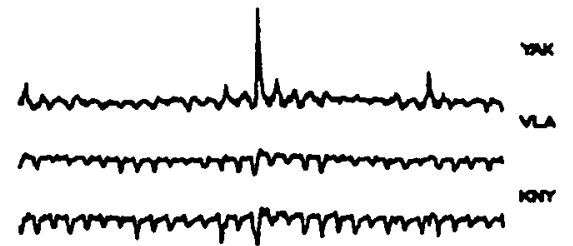

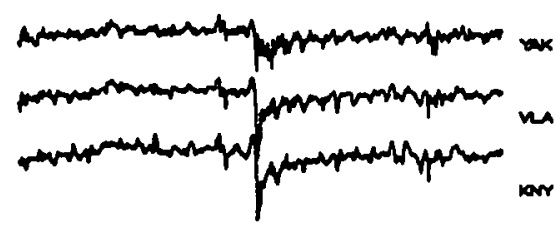

$Y$ Component

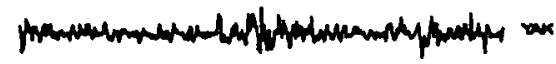

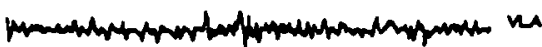

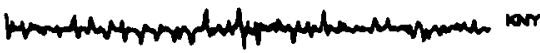
$Z$ component

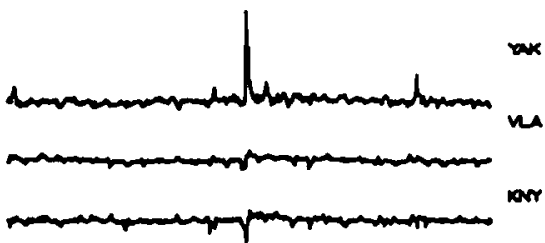


Table 4.6. Correlation before and after removal of daily variation. Observatories displaced mainly in east-longitude. Before - upper triangle, After - lower triangle.

\begin{tabular}{|c||r|r|r|}
\hline X & KIV & SVD & YAK \\
\hline \hline KIV & $*$ & 0.831 & 0.238 \\
\hline SVD & 0.873 & $*$ & 0.558 \\
\hline YAK & 0.599 & 0.751 & $*$ \\
\hline
\end{tabular}

\begin{tabular}{|c||r|r|r|}
\hline Y & KIV & SVD & YAK \\
\hline \hline KIV & $*$ & 0.716 & -0.124 \\
\hline SVD & 0.457 & $*$ & 0.276 \\
\hline YAK & -0.077 & 0.265 & $*$ \\
\hline
\end{tabular}

\begin{tabular}{|c||r|r|r|}
\hline Z & KIV & SVD & YAK \\
\hline \hline KIV & $*$ & 0.568 & 0.019 \\
\hline SVD & 0.737 & $*$ & 0.373 \\
\hline YAK & 0.243 & 0.476 & $*$ \\
\hline
\end{tabular}

Table 4.7. Correlation before and after removal of daily variation. Observatories displaced mainly in co-latitude. Before - upper triangle, After - lower triangle.

\begin{tabular}{|c||r|r|r|}
\hline X & YAK & VLA & KNY \\
\hline \hline YAK & $*$ & 0.735 & 0.419 \\
\hline VLA & 0.765 & $*$ & 0.849 \\
\hline KNY & 0.698 & 0.918 & $*$ \\
\hline
\end{tabular}

\begin{tabular}{|c||r|r|r|}
\hline Y & YAK & VLA & KNY \\
\hline \hline YAK & $*$ & 0.938 & 0.871 \\
\hline VLA & 0.759 & $*$ & 0.972 \\
\hline KNY & 0.574 & 0.864 & $*$ \\
\hline
\end{tabular}

\begin{tabular}{|c||r|r|r|}
\hline Z & YAK & VLA & KNY \\
\hline \hline YAK & $*$ & 0.408 & 0.157 \\
\hline VLA & 0.348 & $*$ & 0.745 \\
\hline KNY & -0.036 & 0.386 & $*$ \\
\hline
\end{tabular}


was found to decrease after the daily variation was removed. This is because the daily variation is the major oscillation within these time series and so when it is reduced the correlation decreases. It was noted that the correlation coefficients for $Y$ were high even after removal. This was due to the large amplitude diurnal variation which was still clearly apparent after the removal method had been applied. The low correlation for the $Z$ component indicates that where gaps occur, values predicted from nearby observatories may not be entirely realistic. As previously mentioned the magnetograms discussed here may be found in Figures 4.6 and 4.7.

\subsubsection{Interpolation for Missing Data}

It was found that of the data selected from the CDROM database approximately $30 \%$ of the observatory records were incomplete. For some stations only one or two hours were missing while at others a day or even longer period had no observations. There are various reasons why gaps may occur in an observatory record. Where older instruments are used, such as the LaCour variometer, failure of the lamp or its trace being too faint during rapid fluctuations, failure to change the photographic paper, the reading going off scale ${ }^{3}$ and interference by small animals ${ }^{4}$ can be the cause of problems. The more recent instrumentation, fluxgate magnetometers standardized with a proton precession total field instrument, is more reliable. However, where data is relayed back to a base over the telephone network, communication can be interupted by lightning strikes (Barraclough, personal communication).

Where gaps occur in recordings it is not possible to predict the true magnetic field. However, it is benefical to have observations at many different locations and therefore as long as the number of missing values did not significantly degrade the storm record estimates of their magnitude were calculated. The scheme used to predict data must work for whatever section of the time series estimates are required. The typical morphology of the storm records are displayed in Figures 4.6 and 4.7, note the records without the daily variation. Time series of this nature could be thought of as being composed of a stationary, stochastic process within which an anomalous transient event occurs. Using statistical methods for forecasting values predictions of the missing data could be made if it were not for the presence of the storm. A technique incorporating knowledge of the time and form of the storm was required. This was achieved using information from other observatories with complete records.

\footnotetext{
${ }^{3}$ Provision is usually made to have a record at reduced scale for large variations.

'Such as spiders in remote rural locations!
} 
Figure 4.8. Relationship of observatories in data generation scheme.

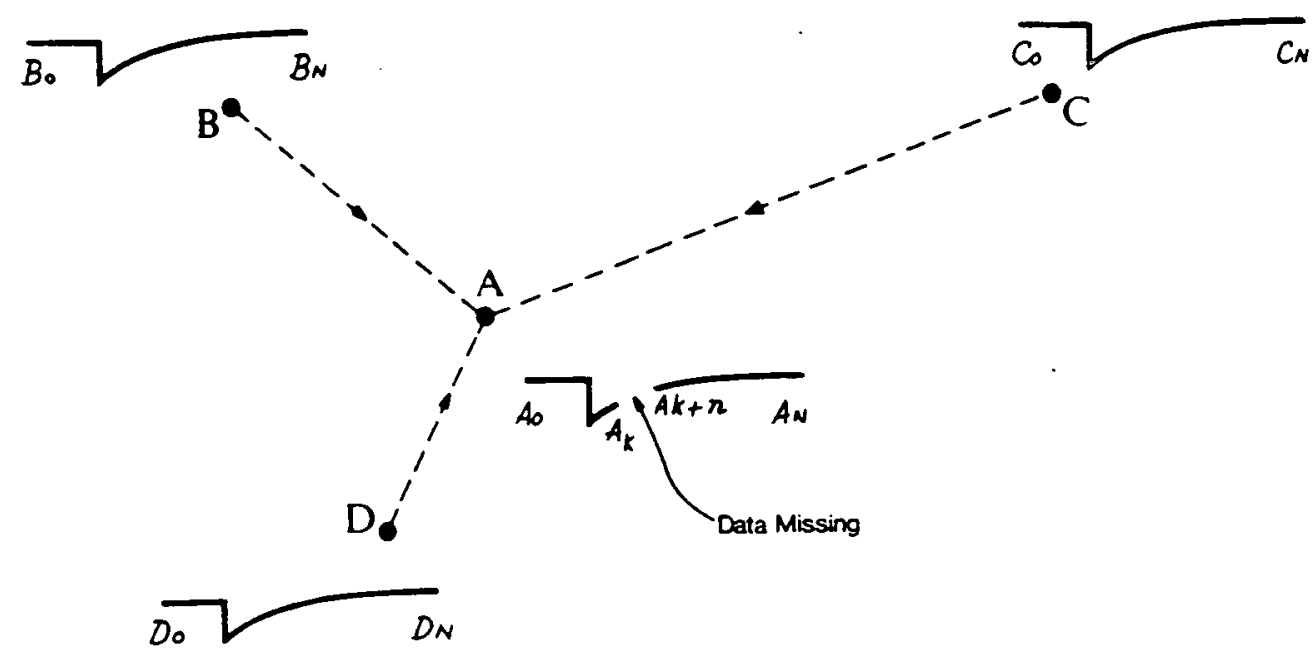

Due to geomagnetic storms being a Universal Time dependent phenomena the relationship between the magnetic field at any two displaced observatories is constant in time. Note that the Local Time dependent daily variation has been removed as far as possible (Section 4.2.3). The relationship will, however, be subject to inevitable discrepancies but an estimate of the variation at one observatory may be produced by multiplying the other observatory record by some constant. If the observatories are not too distant complications due to any local features will be reduced. This approach formed the basis for the means of generating data where gaps occured.

Referring to Figure 4.8 in this case three observatories B,C,D are found within the vicinity of A. Station A has no observations between the values recorded at times $t=k$ and $t=k+n$. Extending the hypothesis presented above, a model of the variation at $A$ is given by the linear combination of the magnetic elements at $B, C$ and $D$

$$
\mu_{t}=b B_{t}+c C_{t}+d D_{t}
$$

where $\mu_{t}$ is the modelled variation at $\mathrm{A}$ and $B_{t}, C_{t}, D_{t}$ represent a magnetic element at the three observatories $B, C, D$, all at time $t$ and $b, c, d$ are the model coefficients. If the coefficients are estimated over the times $\mathrm{A}$ was operating they may then be used to give $\mu_{t}$ when $A_{t}$, the observation of the magnetic element at $\mathrm{A}$, was missing. To 
estimate the three coefficients for the many known observations an error term $\varepsilon_{t}$ must be introduced

$$
A_{t}=b B_{t}+c C_{t}+d D_{t}+\varepsilon_{t}
$$

This term gives the discrepancy between observed and model variations at time $t$. The method of Least Squares (Section 3.3.2) may then be used to give values of the coefficients. Thus the sum of the error term squared is

$$
S=\sum \varepsilon_{t}^{2}=\sum\left(A_{t}-\left(b B_{t}+c C_{t}+d D_{t}\right)\right)^{2}
$$

For the minimum of $S$

$$
\frac{\partial S}{\partial b}=0, \quad \frac{\partial S}{\partial c}=0 \text { and } \quad \frac{\partial S}{\partial d}=0
$$

the resulting equations may be expressed in matrix form

$$
\left(\begin{array}{ccc}
\sum B_{t} B_{t} & \sum B_{t} C_{t} & \sum B_{t} D_{t} \\
\sum C_{t} B_{t} & \sum C_{t} C_{t} & \sum C_{t} D_{t} \\
\sum D_{t} B_{t} & \sum D_{t} C_{t} & \sum D_{t} D_{t}
\end{array}\right)\left(\begin{array}{l}
b \\
c \\
d
\end{array}\right)=\left(\begin{array}{c}
\sum A_{t} B_{t} \\
\sum A_{t} C_{t} \\
\sum A_{t} D_{t}
\end{array}\right)
$$

where the summations extend over the times when data is available at $\mathrm{A}$, ie. $0 \leq t \leq k$ and $k+n \leq t \leq N$. The dimension of the square matrix equals the number of adjacent observatories, which is three in this instance. Solution of 4.6 yields the Least Squares estimates of $b, c$ and $d$. The coefficients may then be inserted in Equation 4.5 when there was no value recorded at $A$. This method of interpolation between observatories was used by Hobbs to generate annual mean values (Shure et al. , 1983).

It was found that the method worked well for the $X$ component as it is generally well behaved over the Earth so that correlation between observatories is high. This cannot be said, however, for the element $Z$ which has a strong local dependence. Due to the poor correlation between $Z$ records the interpolated estimates were often a value near to the mean of the time series, usually $-1,0$ or 1 . The $Y$ component was not considered as it did not figure in the analysis scheme (Chapter 6).

It was noted that the methodology described above makes the assumption that the overall relationship between observatory records does not change throughout the entire time series. However, a constant relationship between observatory magnetograms is not strictly true throughout the interval. During the storm itself the relationship is different from the times not affected by the disturbance and thus the scheme is flawed. However, this did not prevent satisfactory use of the method. Figure 4.9 shows the effect of the interpolation scheme in reproducing data that was temporarily removed. 
Figure 4.8. Comparison between values generated using the Interpolation Scheme and Original data. Interpolated and Original $X$ component data for the Spanish observatory tol. The nearby Iberian observatories coi, $\operatorname{lgr}$ and alm were used in the Interpolation Scheme.

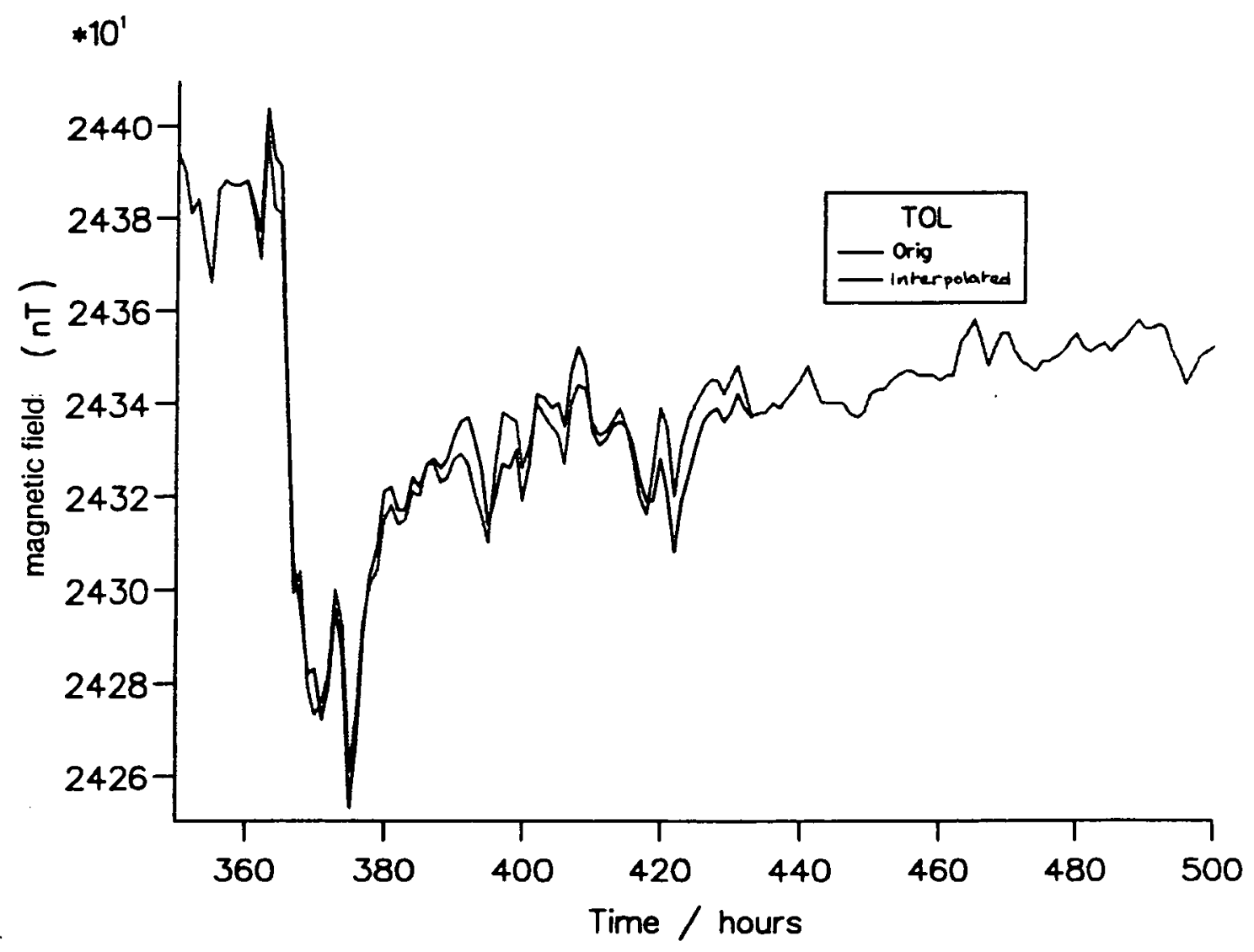


The affect of both the daily variation removal technique and interpolation scheme is displayed by Figures A.2 and A.3 which show magnetograms before and after application of the two processing stages, refer to the appendix. 


\section{Chapter 5}

\section{Storm Analysis - FT}

\subsection{Background}

\subsubsection{The Approach of Analysis}

Refering back to Figure 2.4 it does not matter whether Fourier Transformation or Spherical Harmonic Analysis are carried out first. In the case of this study it was decided to proceed with FT followed by SHA. An advantage of conducting the FT first is that attention may then be focussed on the lower frequencies, the high frequencies may be discarded as they are not relevant to EM studies of the deep Earth. Thus the database is reduced in size from a time series of say 720 hourly values to 30 Fourier coefficients, if frequencies up to $1 \mathrm{cpd}$ are used. This makes storage more convenient and shortens the processing time involved in repeating the SHA. The steps outlined in this chapter were repeated on all 44 storms.

\subsubsection{Preprocessing the Time Series}

Before a time signal is transformed to the frequency domain certain steps must taken. The mean of the time series was subracted from the record to remove power at zero frequency. Usually any overall trend must also be eliminated from the data because of leakage though this was not necessary due to the extremely small secular variation within a month's recording. Chapter 4 concerning the initial treatment of data explains the removal of the periodic daily variation signal. This was undertaken to allow operation of the scheme for generation of missing values (Section 4.2.4) and otherwise would not have been strictly necessary when the SHA was to be carried out only on the low frequency Fourier coefficients. 


\subsection{Calculating the Fourier Coefficients}

Conversion to the frequency domain was carried out using the FFT algorithm developed by R.C. Singleton (Singleton, 1968). This is a mixed-radix routine and therefore the record length does not have to be equal to $2^{N}$ where $N$ is integer. Therefore records could be used as input as they stood without padding out with zeros.

The original Fourier coefficients (fc's) are shown in diagrams (a) to (f) of Figure 5.1, it is evident that the $X$ component is the most energetic. The greatest magnitude coefficents are in general found at the lower frequencies for the $X$ and $Z$ components, though this is not the case for $Y$. The frequency ordinate has been cut off at $3 \mathrm{cpd}$, the fc's decrease further up to the Nyquist frequency of $12 \mathrm{cpd}$. It was noted that the $X$ and $Z$ sequences of coefficients reveal an alternate switching from positive to negative values which seems to be modulated by some envelope function.

The dominant frequencies contained within a magnetic storm extend roughly between 0.04 and $0.5 \mathrm{cpd}$ (Schmucker and Jankowski, 1972) and in this range the magnitude of the real and imaginary $Y$ component coefficients is very small. This is so because of the lack of a recovery phase in the $Y$ trace which implies it is not a feature of magnetic storms thus agreeing with the assumed zonal source (Section 6.3).

It was decided to concentrate attention on the lowest frequency fc's extending up to $1 \mathrm{cpd}$. The estimate at the fundamental Fourier frequency was included in the analysis to observe the response at the lowest obtainable frequency. Using hourly values the highest resolvable frequency is $0.5 \mathrm{cph}$ or $12 \mathrm{cpd}$ which is well above the dominant frequency content of magnetic storms. The magnitude of the $\mathrm{fc}^{\prime} \mathrm{s}$ decreases near to zero a long way before this limit (Figure 5.1) and therefore aliasing, especially contamination below $1 \mathrm{cpd}$, will be very small. Hobbs (1987) used a synthetic storm function, defined in Section 5.4, with and without noise to investigate the reliability of the estimates of the fc's. He found that beyond $1.87 \mathrm{cpd}^{1}$ the fc's were too corrupted to be used indicating a decreasing signal to noise ratio with increasing frequency. Thus it is probable that greater uncertainties are present in the fc's at the $1 \mathrm{cpd}$ end of the used frequency range. The limit of $1 \mathrm{cpd}$ corresponds to the fundamental frequency of the diurnal variation. It has been noted (Schmucker and Jankowski, 1972) that the daily geomagnetic variation is affected by lateral variations in the conductivity of the top-most mantle and crust, and by the ocean-continent contrast and therefore this is an appropriate upper limit. However, this does not mean to say that below this frequency the response is totally unaffected by lateral conductivity variations as there is evidence

\footnotetext{
${ }^{1}$ Length of the time series was 256 hours. $1.872 \mathrm{cpd}$ is then $0.078 \mathrm{cph}$ which equals the 20th Fourier frequency.
} 
Figure 5.1. Fourier Coefficients Before and After Convolution with Blackman-Harris Window (Storm 2, Observatory tkt.). Original fc's, before convolution, diagrams (a) to (f), after convolution (g) to (l). Horizontal Axis: Frequency (cpd),
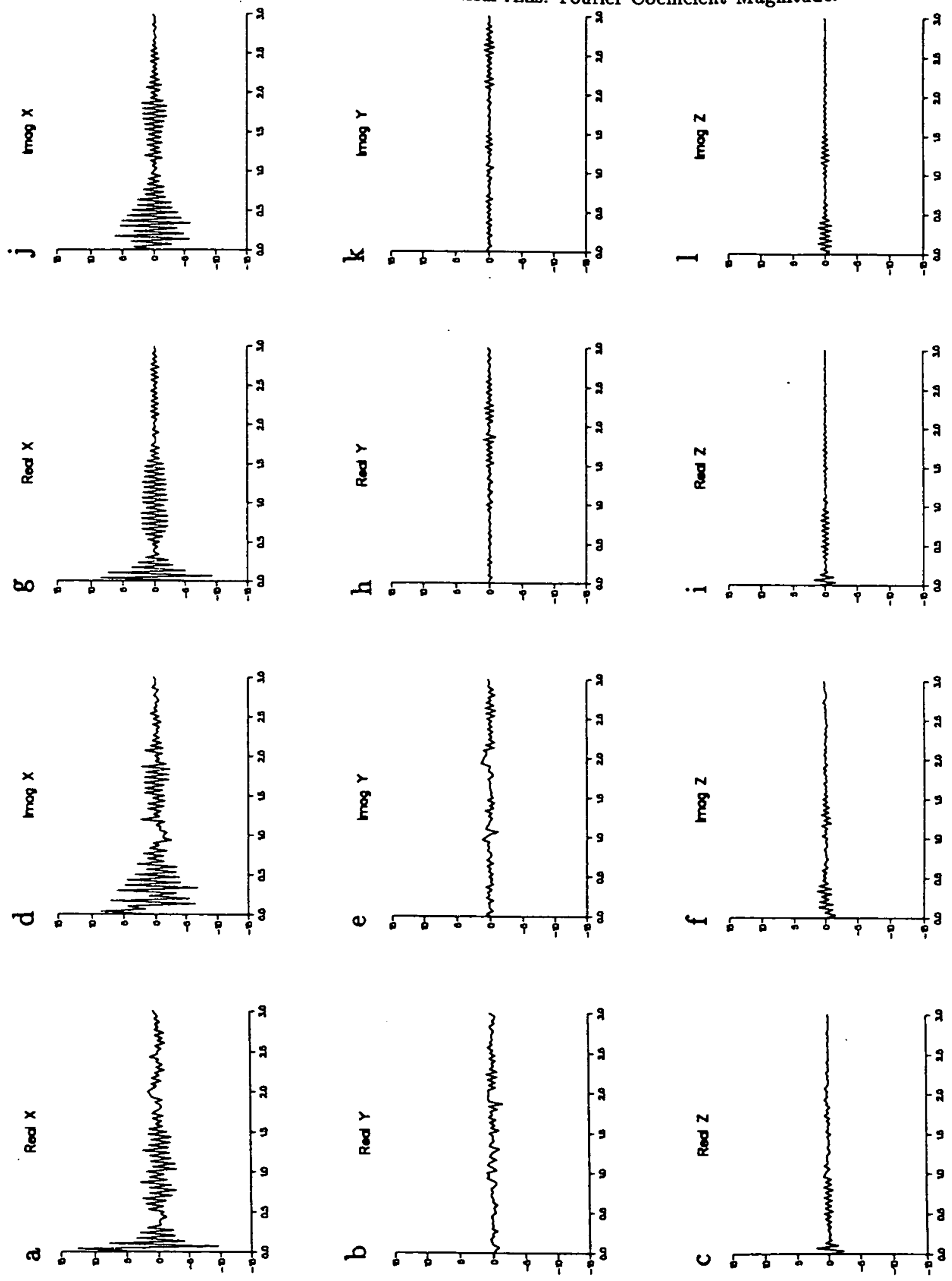
(Roberts, 1984) that some obervatories are influenced, particularly by the oceans, at lower frequencies.

\subsection{Application of the Window Function}

The intervals of data selected for analysis were chosen to position the main phase of the storm near the centre. This was carried out because of the shape of data windows which downweight the signal at the record limits. Therefore the time series were suitable to be used with a smooth data window without significantly reducing the power of the storm.

As mentioned in Section 3.2.2 various window functions exist to improve estimation of the Fourier coefficients. The use of a smooth data window increases reliability of the Fourier coefficient estimates but also decreases the resolution. The window selected for this study is the minimum 3 term Blackman-Harris window (Harris, 1978). It has the desirable property of significantly reducing sidelobes, and therefore bias, while resolution is still satisfactory. This data window and Fourier transform are shown in Figure 5.2. The sidelobes are much smaller than that of the Hanning window (Fig-

Figure 5.2. 3 Term Blackman-Harris data window and Fourier Transform. After Harris (1978).
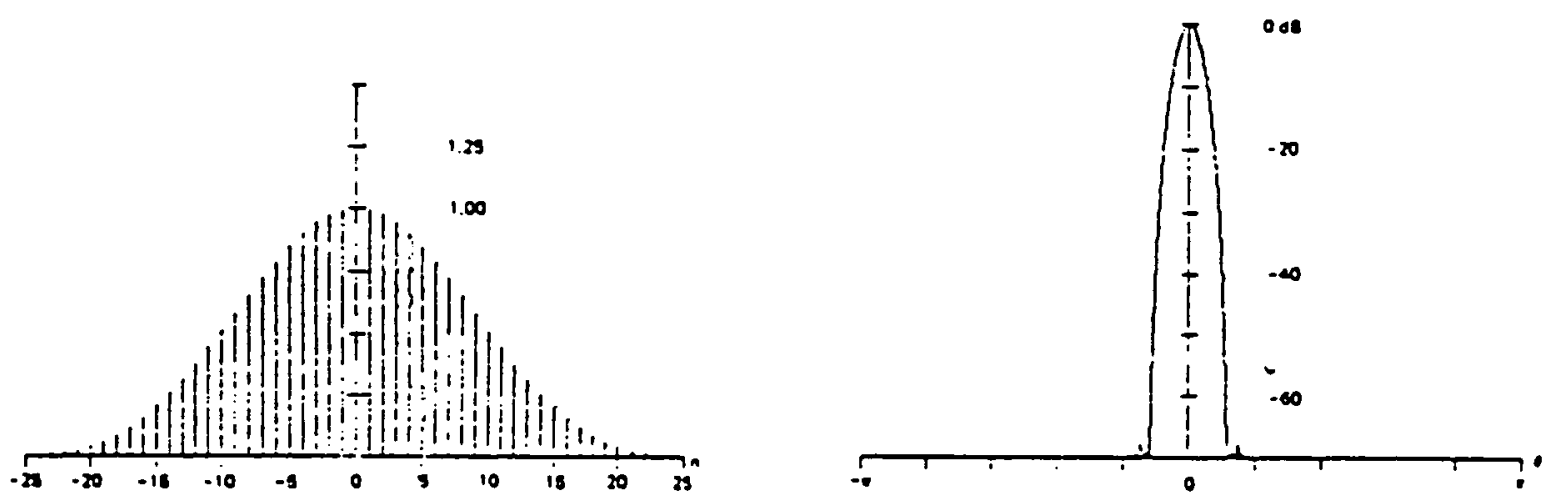

ure 3.2) though the central peak is wider and so resolution poorer. This window was applied in the frequency domain by convolution. The transform of the window may be represented for a DFT sequence by the three coefficients

$$
0.42323,-0.248775,0.03961 \text {. }
$$


The minus sign of the second coefficient arises from shifting the origin of the window to zero time. This was applied in the frequency domain by convolving the Fourier coefficients with a five element array formed from the above three coefficients, reflecting the second and third about the first. An example of the real and imaginary coefficients before and after convolution is given in Figure 5.1. The real and imaginary sequences are made less erratic after the window is applied, i.e. the estimates are more reliable but are less sensitive to individual component frequencies.

Data windows such as the Blackman-Harris window are designed to bring the recorded values down to zero at the edges of the record. However, the storm sequences are by nature reduced near to the limits of the time interval. For this reason using a smooth data window, as opposed to the boxcar, should not be as critical in this study as it would be if the time series was statistically stationary. Leakage has nevertheless been decreased as the windowed diagrams of Figure 5.1, (g) to (1), show a 'cleaner' set of coefficients.

\subsection{The Shift of Storm Commencement}

It was observed that the Fourier coefficients, in particular those for $X$ and $Z$, display an oscillatory behaviour changing between positive and negative and are governed by an envelope. This phenomenon is even more pronounced after convolution with the window ${ }^{2}$, diagrams $(\mathrm{g})$ to $(\mathrm{l})$ of Figure 5.1.

The cause of this switching behaviour is the shift in time from the origin of the data sequence to the storm commencement. If the fc's had been expressed in terms of amplitude and phase the Fourier amplitudes would be no different form a storm beginning at time zero, however, the phase would be changed. To clarify this phenomenon a synthetic study was undertaken. First of all a spike was moved to different positions within a sequence of zeros and FT carried out, this was then repeated with a synthetic function (Hobbs, 1987) designed to mimick a magnetic storm.

Figure 5.3 displays a spike positioned at time zero in a sequence of 720 points ( 0 to 719), diagram (a), and the result of its Fourier analysis (c and e). A synthetic storm function originating at time zero and its Fourier coefficients are shown in the lower diagrams (b), (d) and (f). The power spectrum is also shown for the two functions, diagrams (g) and (h). The FT of the spike is real and constant for all frequencies as the function is even and every frequency is required to represent a spike or delta

\footnotetext{
${ }^{2}$ The clearer pattern is because the convolution operation is acting in the same manner as a low-pass filter would change a time series. Thus the envelope is more evident and the difference in magnitude between adjacent coefficients is reduced.
} 
function. As a result the power spectrum is constant. For the synthetic storm both real and imaginary parts are needed, the coefficients are the DFT sampling of its Fourier Transform given by Hobbs. The power spectrum decreases very rapidly on the log scale of diagram (h).

The spike was then moved (Figure 5.4) first to the 361st value (a) then the 360th (b) then 350 th (c). The spike in the 361st position corresponds to purely real fc's which switch between positive and negative values of constant magnitude (d). Shifting the spike back by one time interval it is observed that both complex quantites are needed in the frequency domain and that sinusoidal envelopes determine the alternating values ( $e$ and $\mathrm{h}$ ). On moving the spike to position 350 the 'period' of the envelope decreases, the real fc's governed by cosine and imaginary by sine. The power spectrum remains as for the spike located at the origin in all three cases.

To explain this behaviour the equation for the DFT (Equation 3.32) may be examined. It is written for realisable data by changing the limits to define a positive interval of time

$$
G_{m}=\sum_{l=0}^{N-1} g_{l} \exp \left[i 2 \pi \frac{l m}{N}\right]
$$

This is a relationship between $\mathrm{N}$ complex numbers $\left[g_{l}\right]_{l=0, \ldots, N-1}$ and $\mathrm{N}$ complex numbers $\left[G_{m}\right]_{m=0, \ldots, N-1}$. However, for the time series recordings of the magnetic field $g_{1}$ is real. Therefore Equation 5.1 may be rewritten expanding the exponential notation to give

$$
G_{m}=\sum_{l=0}^{N-1} g_{l} \cos \left(2 \pi \frac{l m}{N}\right)+i g_{l} \sin \left(2 \pi \frac{l m}{N}\right)
$$

Now if $g_{l}$ is a spike of amplitude $A$ occuring at the beginning of the record $(l=0)$ then $G_{m}=A$, the imaginary part is zero as it depends on sine. If the spike occurs at time $l=N / 2=360$, the 361 st point, $G_{m}= \pm A$. However, if the spike is near to the middle of the record but $l \neq N / 2$ then $G_{m}$ is complex. The real part varies according to the cosine function and the imaginary as sine as $m$ is incremented in the argument $\left(2 \pi \frac{l m}{N}\right)$.

The above observations may be explained in terms of the time-shift theorem, see Bath (1974). This states that if $f(t) \leftrightarrow F(f)$, then $f(t \pm a) \leftrightarrow \exp [ \pm i a 2 \pi f] F(f)$. Thus the Fourier transform of a function shifted in time by $\pm a$ is obtained from FT of the function originating at time zero modified by $\exp [ \pm i a 2 \pi f]$.

The exponential term is responsible for the sinusoidal nature of the fc's of the shifted spike described above. The synthetic storm function

$$
g(t)=a t \exp [-b t]
$$


Figure 5.3. Spike and Synthetic Function at Time Zero. NB. Spike of amplitude 100 occurs at time zero of diagram (a).
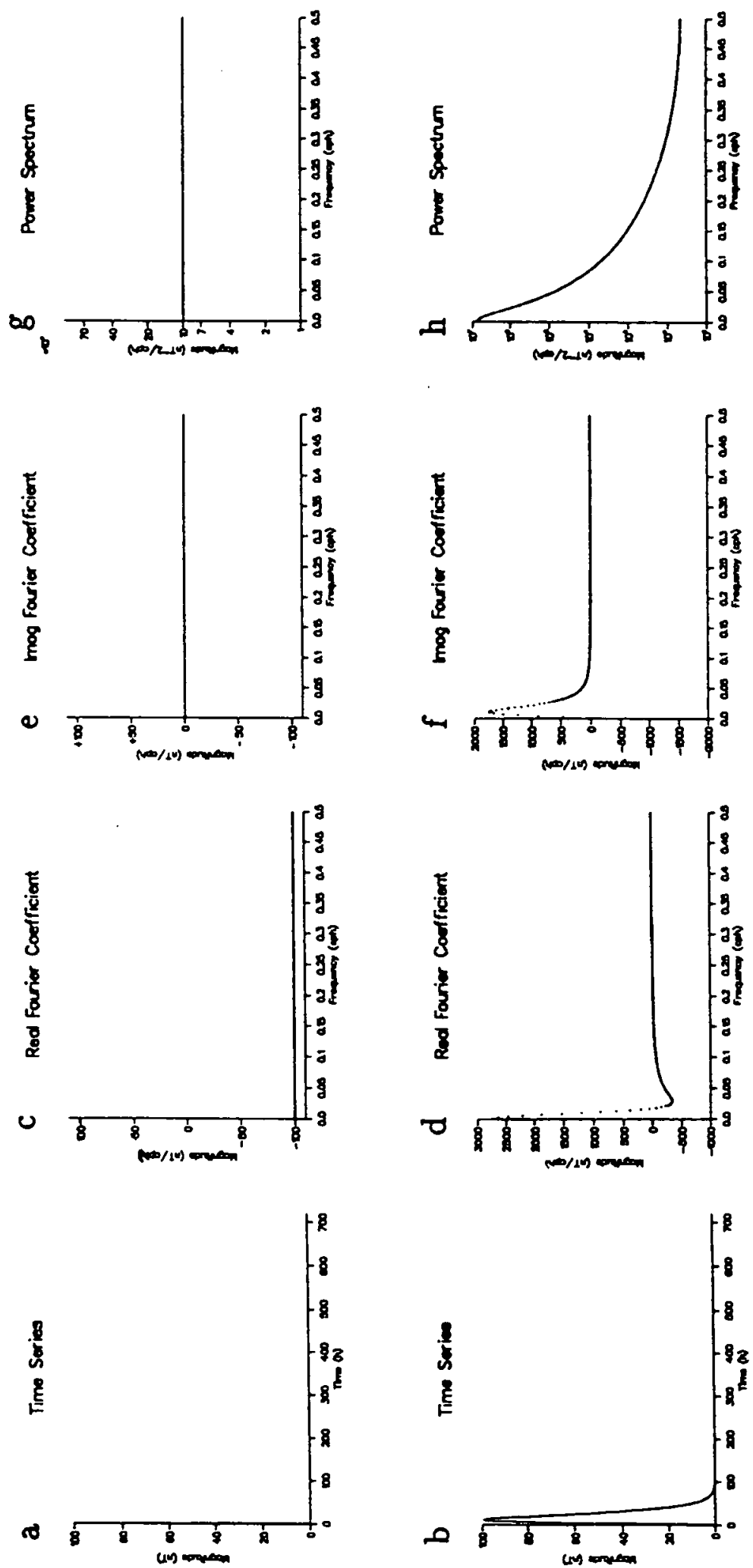
Figure 5.4. Spike located at positions 361,360 and 350.
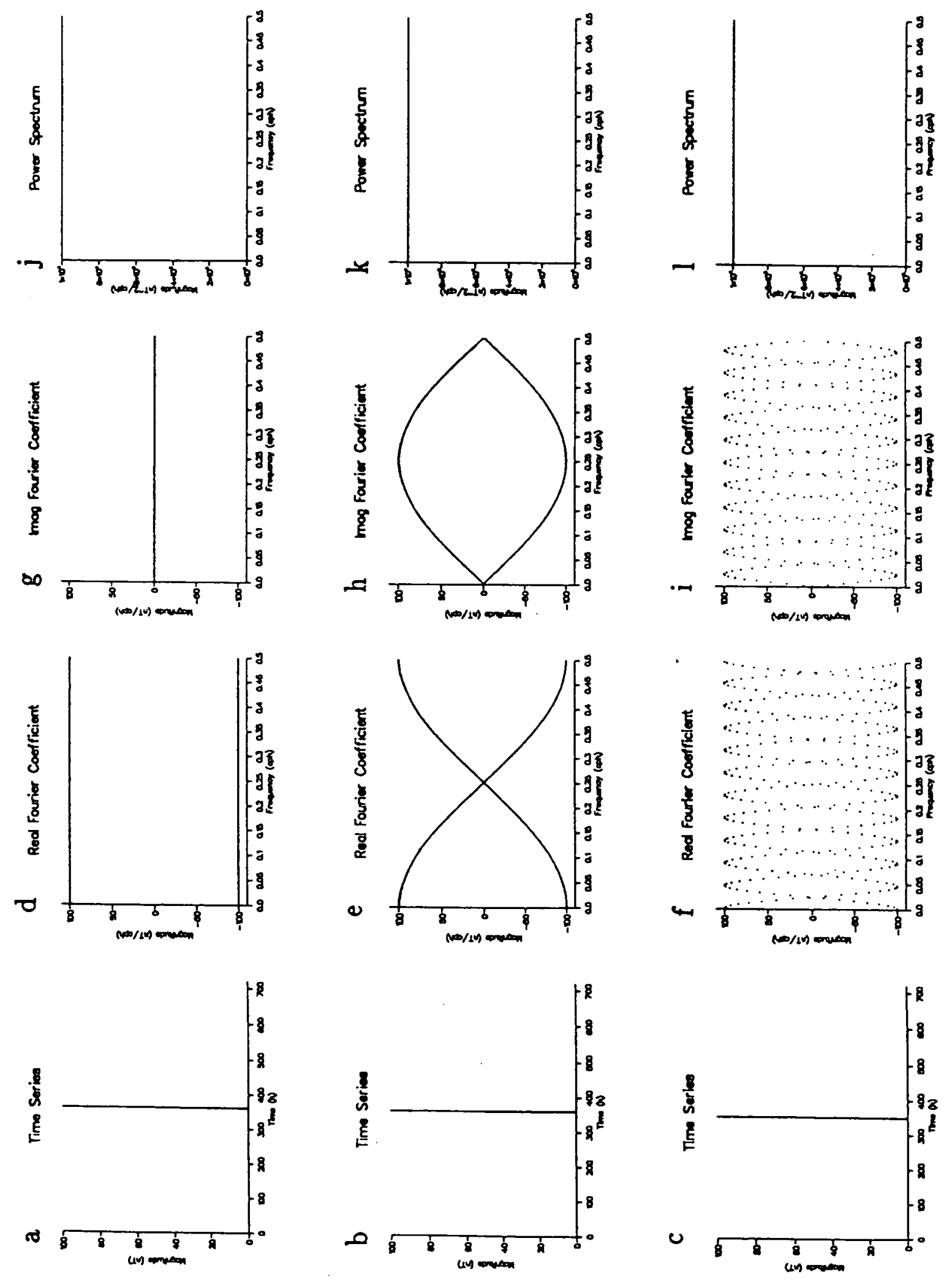
where $a=27, b=0.1$, as in Hobbs, was then made to commence at the same positions as the spike in Figure 5.4, the outcome is shown in Figure 5.5. The diagrams of the fc's in Figure 5.5 (d to i) reveal how the displacement of the function with respect to the origin of the sequence alters the coefficients by incorporating the extra term which modulates their magnitude. However, as $\cos ^{2}+\sin ^{2}=1$ this has no affect on the power spectrum.

The motivation for this synthetic study was the appearance of the fc's of the real data (Figure 5.1). Their behaviour has been copied in the synthetic study which highlighted the phenomenon known by the time-shift theorem. However, as concurrent time intervals were selected from each observatory for every storm, and magnetic storms are Universal Time events, the modulation of the coefficients arising from the shift in time is the same for each record. Thus it does not affect the SHA (Chapter 6) as the fc's at each observatory are affected in the same manner. However, although the modification to the coefficients does not change the relative sizes, if the coefficients at some Fourier frequency approach zero, estimation of the spherical harmonic model will be more difficult in the presence of errors. For this reason a precaution was taken so that very small Fourier coefficients, less than 0.01 magnitude, were not used in the analysis.

\subsection{Multiple Storm Events}

Some of the storm time intervals chosen for analysis contained two events, the disturbance labelled 'Storm 33' is one such example. The three magnetic components from the observatory at L'Aquila (aqu) are plotted in diagrams (a), (b) and (c) of Figure 5.6. It is evident that the power spectrum ( $j, k$ and $l)$ decays with frequency but is also superimposed by a periodic oscillation. The periodic behaviour is not due to the shift in time, which has no affect on the power spectrum, but is a result of two magnetic storms occuring within the time interval. The power spectrum for the $Y$ component decays at a slower rate than the other two components and the modulating period is less pronounced, as previously indicated (Section 5.2) the $Y$ component is not significant in the study of magnetic storms.

The periodic behaviour of the power spectrum is due to a synchronous period in the real and imaginary coefficients, however, the diagrams of the fc's of Figure 5.6 do not indicate this clearly. This was then investigated using the synthetic storm function defined in the preceeding section. Two storms were placed within the data sequence and the affect of Fourier analysis observed. Figure 5.7 gives the fc's and power spectrum for three examples of two storms occuring within the record with different 
Figure 5.5. Synthetic Storm at positions 361,360 and 350 .
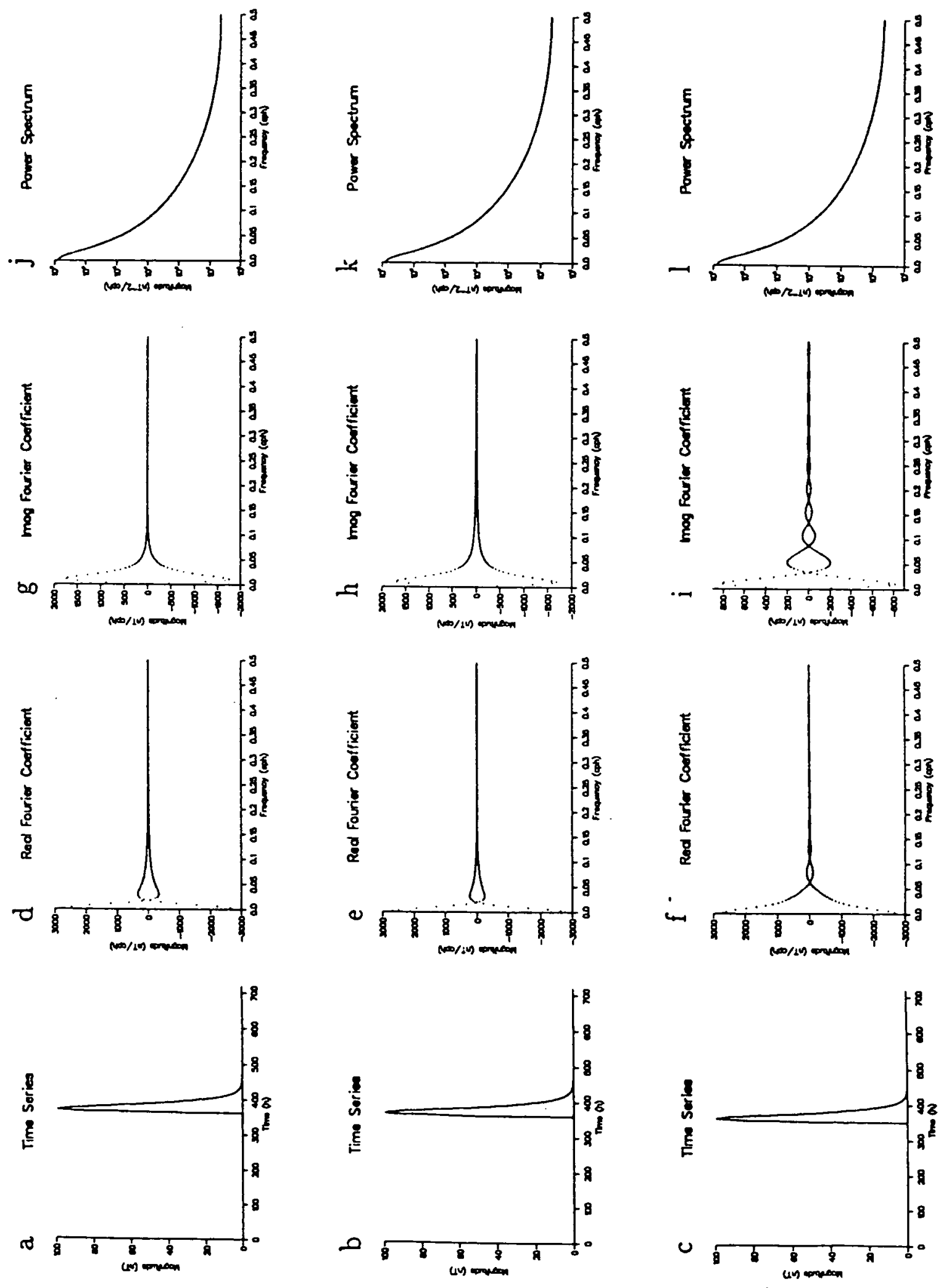
Figure 5.6. Fourier Analysis of recording at L'aquila (aqu), Storm 33.
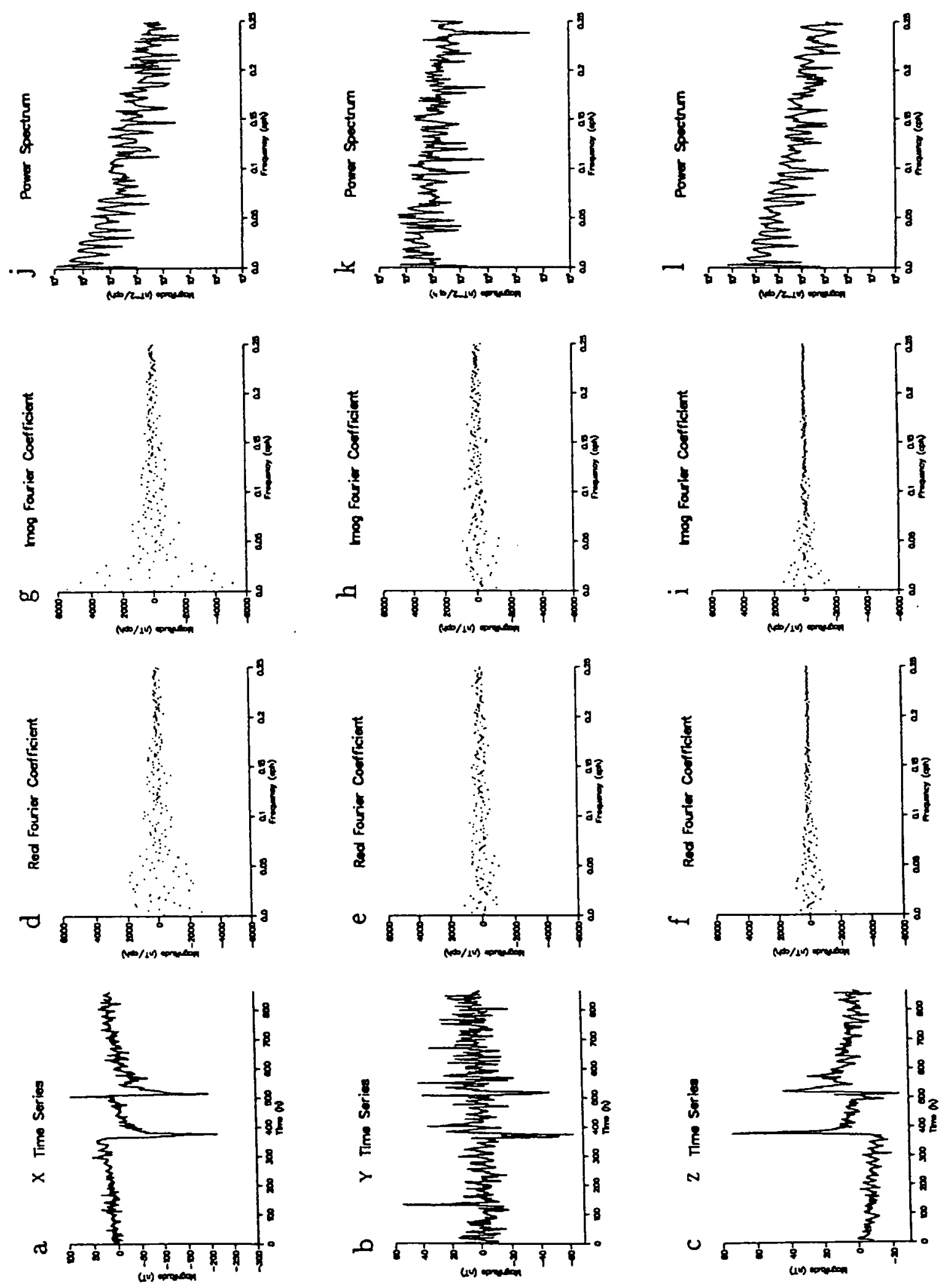
time displacements between them. The real and imaginary fc's of the storms closest together ( $\mathrm{d}$ and $\mathrm{g}$ ) reveal that there is a synchronous period, evident at the lowest frequencies. As the storms are moved further apart ( $b$ and $c$ ) the period becomes shorter, this is shown by the power spectra of (k) and (l). The dependence of the envelope period on event separation is revealed for observed data by comparing storms 9 and 10. Figure 5.8 shows that the closely spaced events of storm 9 give rise to a periodic behaviour of the power spectrum which is of a longer 'wavelength' than that of the more widely separated events of storm 10 .

The described behaviour of the fc's and power spectrum may be explained by the beating phenomena encountered when two vibrations of different frequencies are superposed. The result of this addition is a wave of their average frequency modulated by an envelope with a frequency equal to half the difference between the original frequencies. In this case the 'beating' is observed in the frequency domain caused by the time difference between the two storm events. The resulting envelope is the same for both real and imaginary parts hence the periodic power spectrum.

Records including more than one storm event were included in the analysis. However, when the fc's are reduced by the envelope to values close to zero estimation of the $P_{1}^{0}$ term (Section 6.4) is made more difficult and as stated in the preceeding section the analysis, ie. determination of the response, was not carried out when the fc's were below a set limit. However, it is worthwhile to include such 'storms' to provide information at the Fourier frequencies where the envelope's magnitude is large. 
Figure 5.7. Fourier Analysis of Two Synthetic Storms.
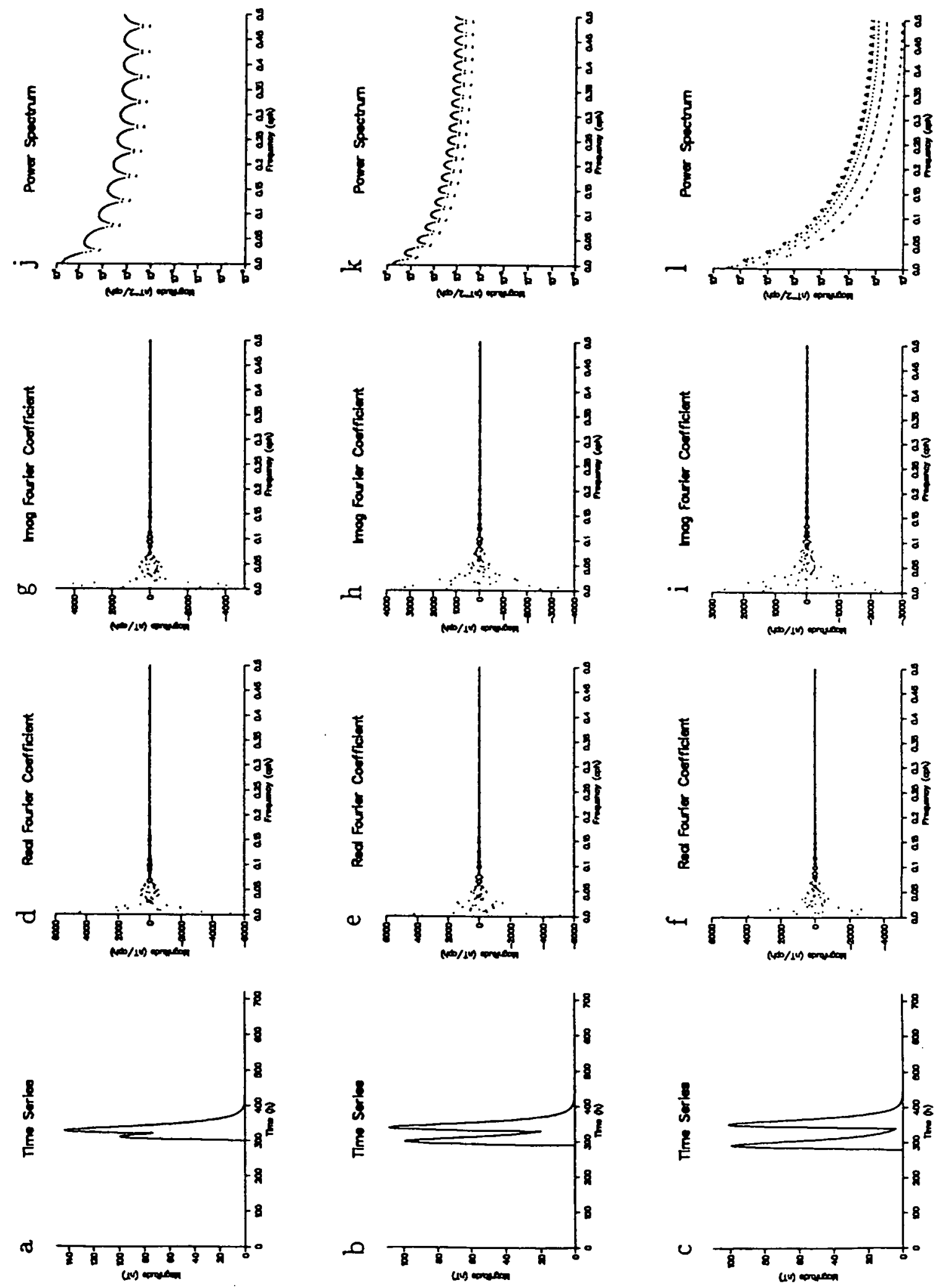
Figure 5.8. Magnetograms and Power Spectra for Storms 9 and 10, L'aquila Observatory (aqu).

Time Series

Storm 9 oqu

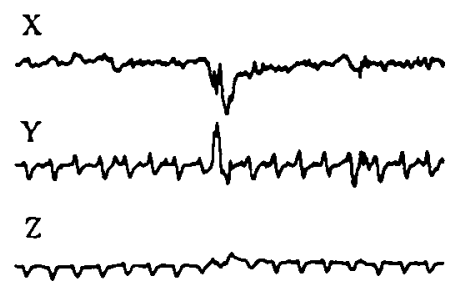

Storm 10 oq

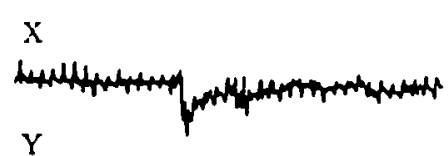

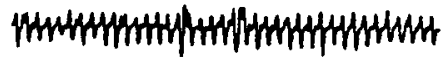

2
$X$ Power Spectrum
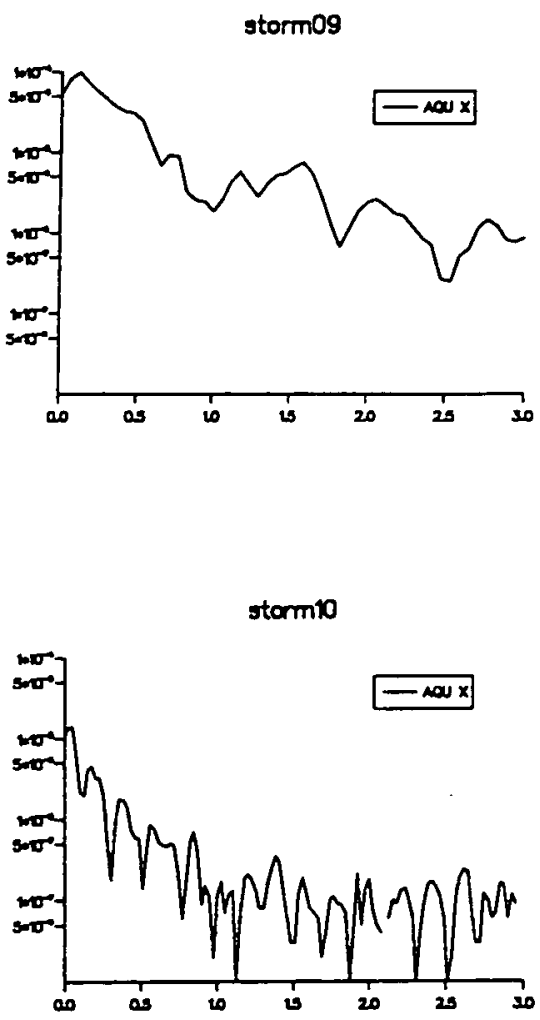


\section{Chapter 6}

\section{Storm Analysis - SHA}

\subsection{The Problem}

The problem of separating the internal and external parts of potential involves finding the 'best' fit of an assumed model to a sparse, unevenly sampled and contaminated set of measurements. This is not a straightforward task. The source of magnetic storms is often defined in terms of a simple $P_{1}^{0}$ spherical harmonic model and although this appears adequate it is an undoubted approximation. Additionally storms occur in the midst of other external geomagnetic phenomena such as the daily variation, polar substorms and the equatorial electrojet. Further complications arise at particular observatories if the Earth's local conductivity is not one dimensional.

Inadequacies in our understanding of the physical system and the simplifications which are then necessary are inevitable. However, the amount of available data also makes the problem even more perplexing. The distribution of observatories around the world is far from even with large areas, mainly in the southern hemisphere, devoid of stations. Only during select time intervals have a large number of the observatories been operating (Figure 4.1) and within magnetograms interruptions are found where no data exists further degrading the dataset. Any analysis of geomagnetic observatory data should therefore not neglect the limitations both in the knowledge of the source field and measurements.

In an effort to determine the most probable global response function 44 individual storms have been subjected to the analysis scheme. It is the aim of this study that by using a large number of storms and finally averaging results any seasonal or anomalous differences will be cancelled out. 


\subsection{Previous Work}

Previous work on magnetic storms (e.g. Anderssen et al., 1970; Devane, 1977; Marshall, 1980) has included attempts to determine the most appropriate spherical harmonic representation of the associated magnetic field. This section gives a summary of the results of this earlier work.

A noteworthy attempt to determine the best model that fits the magnetic field due to magnetic storms is provided by Marshall (1980). He performed SHA in the time domain using zonal harmonics up to $P_{7}^{0}$. This was carried out on the six storms marked with an asterisk in Table 4.2. It was found that the even degree terms were unimportant and to determine whether a model consisting of the odd zonal terms $(1,3,5,7)$ could be further simplified statistical testing was carried out. The test is called the F-test and was initially used in the study of geomagnetic storms by Anderssen et al. (1970); Fougere (1963) had, however, previously applied it to the SHA of the Earth's main field. Working in the time domain on the recovery phase of the six storms Marshall's results were as follows. For the $X$ component, statistically the odd zonal terms up to degree 5 were important in all storms and in three the seventh term was also needed. The F-test on the vertical $Z$ component indicated that the $P_{1}^{0}$ and $P_{3}^{0}$ terms passed the test in all cases and additionally the the $P_{5}^{0}$ term was needed for three of them ${ }^{1}$.

Although terms higher than $P_{1}^{0}$ satisfied the F-test they were much smaller than the first term for both $X$ and $Z$. The stability of the $P_{1}^{0}$ term was assessed by comparing its magnitude between different models. The $P_{1}^{0}$ term in the case of $X$ was found to be relatively independent of the model, however, this was not so for the $Z$ component where unphysical results were obtained. Marshall chose a $P_{1}^{0}, P_{3}^{0}, P_{5}^{0}$ model for $X$ and a $P_{1}^{0}, P_{3}^{0}$ term model to represent the $Z$ component. This compares favourably with Anderssen et al. (1970) who gave $P_{1}^{0}, P_{3}^{0}$ for $X$ and even this was reduced to $P_{1}^{0}$ at some times of the record. For $Z$ they found it was not possible to extract any stable model from the data. It was suggested by Marshall that an inappropriate choice of baseline and time interval, i.e. beginning of storm rather than recovery phase, were responsible for the results of the earlier work. Alternatively it could be that Marshall's analysis has allowed too complex a model. The F-test is a useful statistical tool but it may be that overparameterised models are shown to be valid when noise is present. Marshall refers to this in relation to $Z$, however, his analysis of $X$ may also be too ambitious. He concludes that the F-test should be used as a guide rather than a rigid rule.

The above studies refer to analyses wholly in the time domain. Individual storms

\footnotetext{
${ }^{1}$ Not the same three as required the addition of $P_{7}^{0}$ for the $X$ component.
} 
have been investigated using Fourier Transformation and some results are summarised by Rokityansky (1982; p112). The studies in the forementioned reference agree that the $P_{1}^{0}$ is dominant, the investigations which extended the spherical harmonic model beyond $P_{1}^{0}$ gave unrealiable results.

Therefore from the analysis of individual magnetic storms the $P_{1}^{0}$ term is by far the most important and in some cases was the only term that could be resolved by the data. This is in accordance with the theoretical ring current source (Section 2.3). The result is also supported, but only for the lower frequencies, by work on the magnetic continuum where the $P_{1}^{0}$ term was found to be a satisfactory model for magnetic variations in the frequency range of $0.005 \mathrm{cpd}$ to $0.25 \mathrm{cpd}$ (Banks, 1969) and from 0.01 to $0.2 \mathrm{cpd}$ (Schultz and Larsen, 1983).

\subsection{The Appropriate Spherical Harmonic Model}

In determining the parameters of the assumed spherical harmonic model the conventional least squares technique is commonly used. As stated by Schultz and Larsen (1983) SHA is sensitive to poor data quality which was the reason they took an alternative approach. If a complex spherical harmonic model is sought methods do exist which attempt to improve evaluation of the parameters. Use of the F-test to determine the significant terms of a spherical harmonic model was examined by Brett (1988) though she found little improvement over the ordinary Least Squares method. In the same study an inversion method incorporating a priori information (Gubbins, 1983) was investigated and found to give worthwhile benefits.

However, a high degree SHA does not appear to be required in the analysis of magnetic storm variations. Therefore, initially an ordinary LS analysis was carried out to determine the approximate spatial form of the data. At each Fourier frequency SHA was conducted on the observatory Fourier coefficients up to degree and order 4. This limit was suitable to generate a range of spherical harmonics to compare the $P_{1}^{0}$ term with and allowed any storm to be investigated with 25 or more observatories. The result on storm 18 is displayed by the diagrams of Figure 6.1 , for the horizontal components, and Figure 6.2 for the vertical component. Figures A.4, A.5, A.6, A.7, A.8 and $A .9$ in the appendix give the equivalent plots for storms 6,10 and 31 . These were not chosen for any particular reason other than they represent storms recorded by different numbers of observatories and span a representative period of the storms selected (Table 4.2). However, the choice that two of them were examined by Marshall (1980) was deliberate. The forementioned figures plot the 'magnitude' for each spherical 
harmonic function calculated as

$$
\left|P_{n}^{m}\right|=\sqrt{\left(A_{n}^{m}\right)^{2}+\left(B_{n}^{m}\right)^{2}}
$$

where $A_{n}^{m}, B_{n}^{m}$ are the complex spherical harmonic coefficients of cosine and sine geomagnetic east-longitude. Analysis of the horizontal, $X$ and $Y$, components shows that the $P_{1}^{0}$ term dominates the shc's for virtually the whole of the considered frequency range. There is an overall trend for the $P_{1}^{0}$ term to decrease in magnitude for the higher Fourier frequencies and Figure 6.1 shows that $P_{1}^{0}$ is no longer the largest term at $1 \mathrm{cpd}$. The non $P_{1}^{0}$ shc's are of comparable amplitude to each other. There is no evident trend with frequency and therefore these other terms become relatively more important and the $P_{1}^{0}$ coefficients less so towards higher frequencies. From the diagrams of Figure 6.1 and the magnitude of the shc horizontal components of the storms in the appendix there is no evidence to suggest that the $P_{3}^{0}$ term is any more significant than the other terms higher than $P_{1}^{0}$. The study of the $Z$ component (Figure 6.2 and $Z$ shc power plots in the appendix) is of particular interest as the $P_{1}^{0}$ coefficient does not attain the greatest value at any frequency. Generally the most power is shared between the shc's of degree 2 and 3, though appreciable levels occur at degree 1 and 4 . There is some indication that on going to higher frequencies a reduction in magnitude occurs. Another major point concerning the $Z$ component is that the power is very large for storms 18 (Figure 6.2) and 31 (Figure A.9) which are both defined by less than 30 observatories.

From this investigation the $X$ component is clearly dominated by the $P_{1}^{0}$ term especially at the lower frequencies. The same cannot be said for the $Z$ component which appears to be composed of a range of shc's. Although this analysis of the $Z$ component does not bring out the significance of the $P_{1}^{0}$ term it may be that SHA to degree and order four is inappropriate. The diagrams of Figures 6.3 and 6.4 show fc's at specific Fourier frequencies plotted against co-latitude. It is evident that on neglecting any variation in east-longitude there is evidence of a $P_{1}^{0}$ term in both the $X$ and $Z$ components. Similar plots at the Fourier frequencies up to $1 \mathrm{cpd}$ for other storms also support the presence of a $P_{1}^{0}$ signal in $Z$. Therefore despite the extended analysis to degree and order four which does not indicate a strong $Z P_{1}^{0}$ term, inspection of Figures 6.3 and 6.4 would suggest on the contrary that there is a significant underlying $Z P_{1}^{0}$ spatial dependence. If this is so the apparent failure of any $P_{1}^{0}$ structure in this SHA study must be addressed. The relative amplitudes of the fitted $P_{1}^{0}$ terms, Figures 6.3 and 6.4 , show that the sine function determined by the $X$ fc's is roughly three times larger than the cosine function given by the $Z$ fc's. This is expected as 
Figure 6.1. Magnitude of the Spherical Harmonic Coefficients for the Horizontal Components of Storm 18. Horizontal Axis: $P_{n}^{m} n$ first digit, $m$ second (eg. $P_{1}^{0}$ equals 10). Vertical Axis : Magnitude (nT).
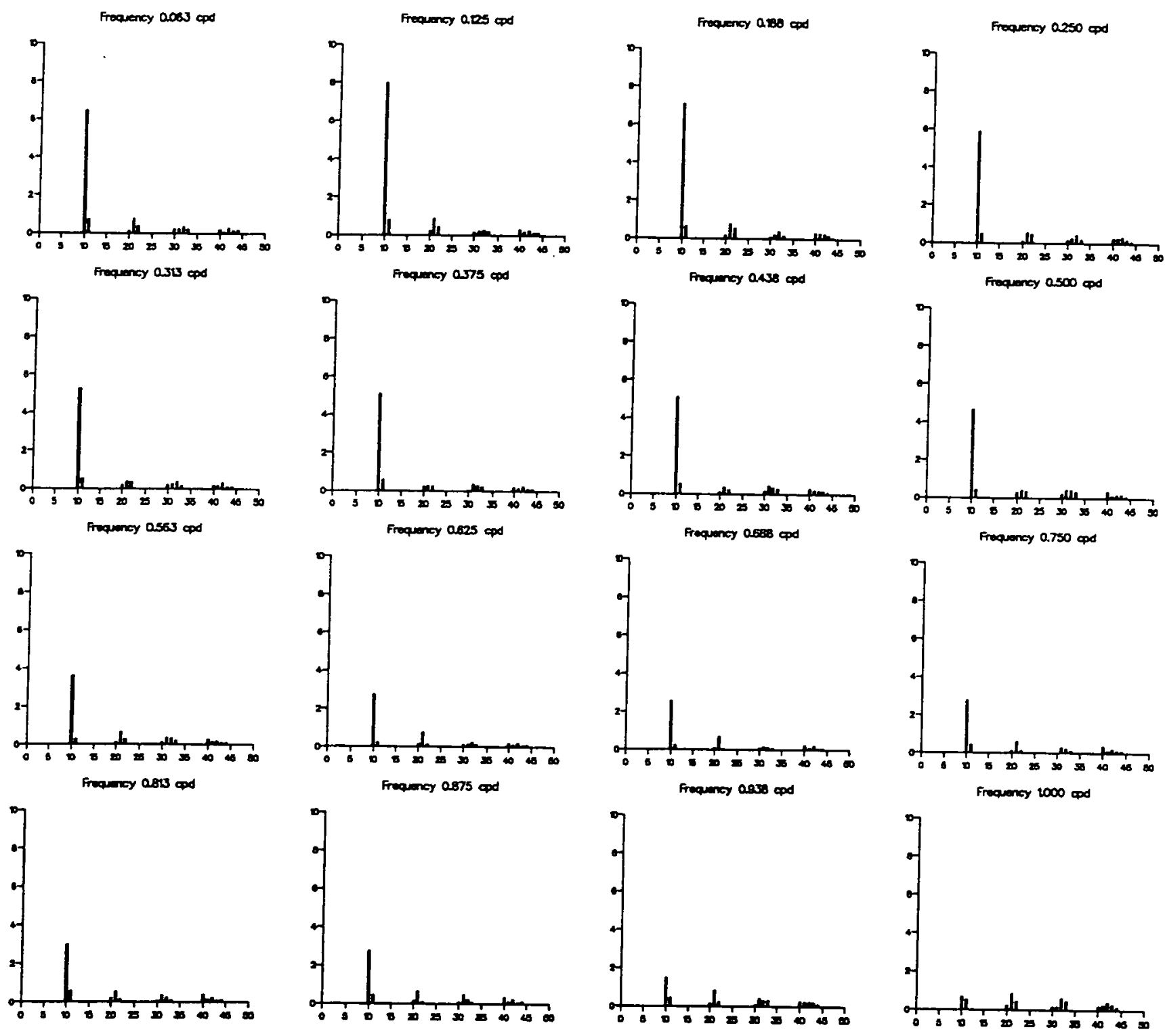
Figure 6.2. Magnitude of the Spherical Harmonic Coefficients for the Vertical Component of Storm 18. Horizontal Axis: $P_{n}^{m} n$ first digit, $m$ second (eg. $P_{1}^{0}$ equals 10). Vertical Axis : Magnitude (nT).
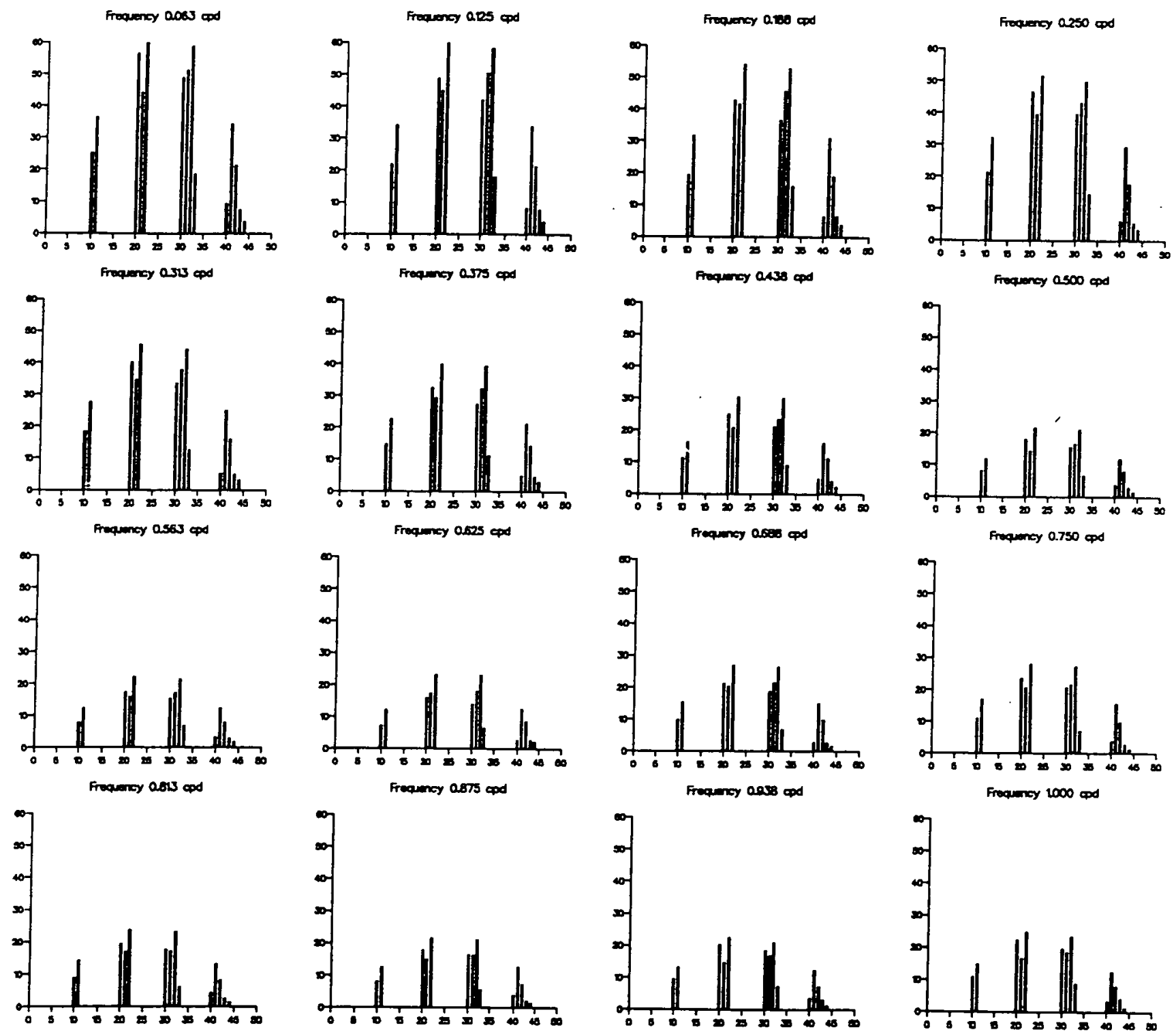
referring to Equations 6.12 and 6.13 a ratio $\beta_{X}: \beta_{Z}$ of $3: 1$ gives $i / e$ of almost 0.3 which is physically acceptable and of the order found by previous workers (Marshall, 1980; p111). The smaller amplitude signal in $Z$ results in a comparatively poor signal to noise ratio in $Z$ when compared to $X$ assuming equivalent noise levels. The amount of random noise, however, is expected to be greater in the $Z$ than in the $X$ component due to difficulties in measuring $Z$ with the older LaCour instruments. Therefore the signal to noise ratio in $Z$ will be even more degraded in relation to $X$. In addition as sine reaches its maximum and cosine its minimum within the range $35^{\circ}$ to $145^{\circ}$ the signal to noise ratio is further downgraded. These points are discussed by Banks (1969), Marshall (1980) and Roberts (1982). It is supposed that the SHA to degree and order four carried out on the $Z$ fc's favours the higher spherical harmonic terms due to the amount of noise and that for this reason the $P_{1}^{0}$ contribution does not appear to be important.

The non- $P_{1}^{0}$ dominated $Z$ fc's could also be due to lateral variations within the mantle. From Equations 6.12 and 6.13 the $Z$ component depends more on the induced internal part of the field while the $X$ component is more attributable to the external part. Thus very simply the $X$ component may be regarded as representing the external source and the $Z$ component the internal induced field. The plots of Figures 6.1 and 6.2 could then be interpreted as showing a $P_{1}^{0}$ source and an induced non- $P_{1}^{0}$ internal field. This would imply that lateral variations in conductivity within the mantle were responsible for the distribution of power shown by Figure 6.2 and the equivalent plots for $Z \mathrm{fc}$ 's in the appendix. It is most likely that some lateral variations in conductivity exist which will reduce the suitability of the $P_{1}^{0}$ term as a model for $Z$, however, as an underlying $P_{1}^{0}$ term is shown in Figures 6.3 and 6.4 it is probably the signal to noise ratio in $Z$ which prevents the emergence of the first harmonic in the extended SHA. It would be of interest to reduce the SHA to include fewer terms so that the stability of the spherical harmonic expansion could be assessed. The non-orthogonal geographical distribution will also be a factor in the reliablility of the SHA.

A further consideration which may help explain the discrepancy between the SHA and pure $P_{1}^{0}$ fit (Section 6.4.3) to the fc's is the nature of the source field near to the limits of the co-latitudinal range, $35^{\circ}$ to $145^{\circ}$. In Figure 6.3 the $P_{1}^{0}$ term is mainly manifest in the imaginary part. Comparing the fc's of imaginary $X$ and $Z$ (b and d) there are a greater number of anomalous fc's near to the ends of the interval in the case of $Z$. Referring to Figure 6.4 it is also found that spurious values near to the co-latitude extremes depart from the overall latitudinal behaviour more so in $Z$ than $X$. Non- $P_{1}^{0}$ spatial structure in the fc's near $35^{\circ}$ and $145^{\circ}$ co-latitude is due to the currents flowing in the auroral oval which have some influence on the total field at the forementioned 
co-latitudes. The limits of $35^{\circ}$ and $145^{\circ}$ co-latitude were adopted to avoid such effects but the plots of the fc's verses co-latitude indicate that it may have been better to restrict the interval to a narrower range even though fewer observatories would then be available. It is probable that auroral currents produce a field which is greater in the vertical component near the extreme co-latitudes of this study and therefore give more anomalous values in $Z$ than $X$. These anomalous values would introduce power into the higher terms of the SHA whereas the robust $P_{1}^{0}$ fit (Section 6.4.3) would hardly be influenced. Therefore complications in the source field that in particular effect the $Z$ component may add to the apparent lack of a $P_{1}^{0}$ term in the $Z$ SHA study.

Therefore from the plots of the SHA up to degree and order four, Figures 6.1 and 6.2 , the only conclusion that can satisfactorily be made is that there is a strong $P_{1}^{0}$ term describing the spatial variation in the $X$ component. The evidence of Figures 6.3 and 6.4 suggest there is also underlying $P_{1}^{0}$ structure in the $Z$ component and it was therefore decided that it would be valid to make the simplification that magnetic storms were adequately modelled by the single $P_{1}^{0}$ term. Thus an estimate of the global $P_{1}^{0}$ response was pursued from analysis of the 44 storms.

A simple model is advisable in the analysis of storms recorded by different sets of observatories. It was found in the investigation of the $Z$ component of storms 18 and 31 , recorded by a low number of stations, that the shc's are very large. This is due to the calculations being unstable as the number of points is not much larger than the number of model parameters to be found. Brett (1988) discusses this problem stating that:

In the particular case of spherical harmonic analysis, underdeterminacy can appear in an overdetermined problem if the data positions fail to constrain the high values of a harmonic or distinguish sufficiently between different harmonics.

Thus, although the problem appears to be overdetermined the data are not sufficient for the complexity of the model and an unstable result is found. This problem is very much reduced when the spherical harmonic model only involves one parameter, allowing storms to be incorporated in the analysis with fewer observatories.

\subsection{Robust Estimation of the $P_{1}^{0}$ term}

\subsubsection{Introduction}

An argument has been presented in Section 6.3 that the SHA is reduced to determining solely the coefficient of the $P_{1}^{0}$ term. Therefore from Equations 3.38 and 3.43 the spatial 
form of the $X$ and $Z$ components follows

$$
\begin{gathered}
X=-\beta_{x} \sin \theta^{\prime}, \\
Z=\beta_{z} \cos \theta^{\prime},
\end{gathered}
$$

where $\beta_{x}$ and $\beta_{z}$ are the coefficients of the assumed model and $\theta^{\prime}$ is geomagnetic colatitude. Thus the fc's of the $X$ and $Z$ components are expected to behave simply as $-\sin \theta^{\prime}$ and $\cos \theta^{\prime}$ respectively.

Examples of the fc's at a specific Fourier frequency plotted against observatory geomagnetic co-latitude are given in Figures 6.3 and 6.4. It is evident that in general the relationships 6.1 and 6.2 governing the $X$ and $Z$ components are obeyed though there is some spread about the predicted spatial form. There are, however, a few isolated fc estimates which do not follow the form indicated by the other estimates. Anomalous values which are drawn from a different distribution are known as outliers and it may be that these wayward points are examples.

The ordinary LS method for determining the model fit (Section 3.3.2) depends on minimising the sum of the residuals squared. If the estimates are normally distributed about the predicted model then this is the optimum method. However, outlying values produce large residuals which have a much greater affect on the result than those which are more in accord with the model. A bias in favour of the outliers occurs. This bias in the estimate of the model coefficient may be reduced if an alternative to the pure LS scheme is used. To achieve this aim the problem must be approached by employing robust statistical techniques which reduce the influence of outlying data points. An overview of robust methods is given in the book edited by Hoaglin et al. (1983), the application of robust procedures to geophysical problems includes the work of Egbert and Booker (1986) and Chave et al. (1987).

\subsubsection{Robust Methodology}

The standard LS problem involves the minimisation of

$$
\left[\int_{-\infty}^{\infty}|x-\bar{x}|^{2} d x f(x)\right]^{1 / 2}
$$

which is known as the $L_{2}$ norm, $f(x)$ is a continuous probability density function. The result of this process gives the definition of the mean $\bar{x}$. The effect anomalous values have on the estimation of model parameters is reduced if instead of minimising the sum of the residuals squared, the sum of the absolute value of residuals is minimised. 
Minimisation of the norm

$$
\int_{-\infty}^{\infty}|x-\tilde{x}| d x f(x)
$$

results in finding the sample median $\tilde{x}$, this is the $L_{1}$ norm.

It may therefore be appropriate to use the $L_{1}$ norm as the basis of determining the 'best' fit to a set of outlier contaminated data. However, there are arguments opposed to the use of $L_{1}$ statistics (Chave et al., 1987). The main objection is that in most cases only a fraction of the residuals significantly violate the normal distribution which is the distribution associated with the $L_{2}$ norm. The more complex statistics of the $L_{1}$ norm are not applicable to the majority of the data and it therefore seems better to base estimation on the $L_{2}$ norm.

The standard LS method could be made less sensitive to outliers if these points were given a smaller weight than those values which indicated a common model. However, unless some a priori information exists it is not possible to assign weights to the original data. An initial ordinary LS model must be fitted to the data to allow calculation of the residuals. The resulting residuals may then be used to determine individual weights for each point. This method of calculating weights based on an earlier model fit requires the process to proceed iteratively. The preliminary model will be biased in favour of the outlying values, however, as these are downweighted at each successive iteration their influence on the model is reduced. In this way, as long as the majority of the data is 'good', a more representative model is found.

The outline described above is the robust method known as Iteratively Reweighted Least Squares (IRLS). The procedure is related to M-estimators (Hoaglin et al., 1983; p341) which seek to minimise some general function of residuals. The function of residuals is called the loss or objective function, denoted $\rho\left(r_{i}\right)$, where $r_{i}=Z_{i}-\beta_{z} \cos \theta_{i}$ in the case of the vertical component. Thus taking the $Z$ component fc's $\left(Z_{i}\right)$ as the example it is desired to find the value of $\beta_{z}$ that minimises the following expression

$$
\sum_{i=1}^{N} \rho\left(Z_{i}-\beta_{z} \cos \theta_{i}^{\prime}\right)
$$

where $N$ is the number of observatories and the other terms are as in Equation 6.2. The methodology presented here is based on Egbert and Booker (1986). The minimum of the sum 6.3 is given when

$$
\sum_{i=1}^{N} \psi\left(Z_{i}-\beta_{z} \cos \theta_{i}^{\prime}\right) \cos \theta_{i}^{\prime}=0
$$

where $\psi(r)=\rho^{\prime}(r), \psi(r)$ is called the influence function. A weight function $w(r)$ is 
then found where

$$
w(r)=\frac{\psi(r)}{r}
$$

It is then possible to rewrite Equation 6.4 using $w\left(r_{i}\right)$ in place of $\psi\left(r_{i}\right)$ and $r_{i}=$ $Z_{i}-\beta_{z} \cos \theta_{i}^{\prime}$ to give

$$
\sum_{i=1}^{N} w\left(r_{i}\right)\left(Z_{i}-\beta_{z} \cos \theta_{i}^{\prime}\right) \cos \theta_{i}^{\prime}=0
$$

Equation 6.5 may then be solved for $\beta_{z}$.

$$
\beta_{z}=\sum_{i=1}^{N} \frac{w\left(r_{i}\right) Z_{i} \cos \theta_{i}^{\prime}}{w\left(r_{i}\right) \cos ^{2} \theta_{i}^{\prime}}
$$

Thus the present estimate of $\beta_{z}$ is dependent on the residuals calculated from the previous model. The process is made to proceed iteratively until a stable value of $\beta_{z}$ is found.

In the case of LS the loss function is given by $\rho(r)=r^{2} / 2$, which gives an influence function $\psi(r)=r$ and therfore a weight function equal to unity. Therefore setting $w\left(r_{i}\right)=1$ where $i=1, \ldots, N$ in Equation 6.6 gives the ordinary LS estimate of location $\beta_{z}$. This is the primary step in the IRLS procedure. Once this has been accomplished the residuals between the 'observations', the fc's, and the initial model may be calculated. It is then necessary to use some rule to produce weights which are smaller for the outlying fc's that produce large residuals.

It is necessary to introduce some estimate of scale to provide the basis for determining whether a residual is large. Therefore rather than the residual $r$ the scaled residual $u$ should be used

$$
u_{i}=\frac{Z_{i}-\beta_{z} \cos \theta_{i}^{\prime}}{\sigma_{r}}
$$

where $\sigma_{r}$ is some robust measure of scale.

It is then possible to define general weight functions with the intention of reducing the effect of anomalous values. A number of different weighting algorithms are given in Table 11-1 of Hoaglin et al. (1983; p366), the original loss function and the influence function are included.

An attempt at reducing the bias towards large values while assuming a normal distribution for the majority of data was introduced by Huber (1964). He proposed the following defintion for the weight function

$$
w\left(r_{i}\right)= \begin{cases}1 & \left|u_{i}\right| \leq k \\ k /\left|u_{i}\right| & \left|u_{i}\right|>k\end{cases}
$$


where $k$ determines the transition point. These weights correspond to $L_{2}$ norm minimisation for scaled residuals less than $k$ and $L_{1}$ minimisation for larger scaled residuals. Thus although outliers still have some bearing on the model fit their influence is reduced. Weight functions that set large outliers to zero are called redescending functions. The biweight of Tukey (Mosteller and Tukey, 1977) is an example,

$$
w\left(r_{i}\right)= \begin{cases}\left(1-u_{i}^{2}\right)^{2} & \left|u_{i}\right| \leq 1 \\ 0 & \left|u_{i}\right|>1\end{cases}
$$

Outliers for which $\left|u_{i}\right|>1$ are then completely rejected.

The denominator of Equation 6.7 is a robust estimate of scale. The scale of a distrubution is a measure of its width or dispersion, the most commonly used is the standard deviation $\sigma$. An estimate of scale that is more resistant to outliers than the standard deviation is the sample fourth spread or theoretical interquartile range (Section 6.4.4). The median is the central value when the observations are sorted into ascending order. Dividing the distribution further the fourths are the values halfway between the median and the two extremes (Hoaglin et al., 1983; p35). The fourth spread estimate of scale $\sigma_{f}$ is then the difference between the upper and lower fourth. For the Gaussian distribution the fourth spread equals $1.349 \sigma$, therefore a robust psuedo standard deviation $\sigma_{r}$ assuming the Gaussian distribution holds for the majority of data is

$$
\sigma_{r}=\frac{\sigma_{f}}{1.349}
$$

If the data conforms to a Gaussian distribution $\sigma_{r}=\sigma$.

Robust estimators are designed to use the good data values in preference to the poor data. However, there is a limit as to the proportion of outliers for which the estimate is still resistant. The breakdown bound (Hoaglin et al., 1983; p357) of an estimator is the fraction of data that may take any value, without limit, while leaving the location estimate bounded. The estimators described above have a breakdown bound approaching 0.5, the largest possible, provided the robust scale is as, or more, resistant. The estimate of scale suggested is the fourth spread which reduces the value to 0.25 .

\subsubsection{Robust Determination of the $P_{1}^{0}$ Coefficient}

The robust method of Iteratively Reweighted Least Squares is presented in the previous section. This scheme was applied to the observatory fc's for all the magnetic storms under consideration up to a frequency of $1 \mathrm{cpd}$.

Initially the biweight formula (Equation 6.9) was used to weight residuals, these 
weights have been used to robustly determine palaeomagnetic apparent polar wander paths (Thompson and Clark, 1982). However, it was found that in some instances the iteration process did not converge. This is a problem of redescending estimators of location which may give multiple solutions (Hoaglin et al., 1983; p387). Additionally the biweight algorithm does not assign the most appropriate weight to 'good' data points causing a distorted estimate (Chave et al., 1987).

Multiple solutions may be avoided if weights derived from a monotone influence function are used, the solution of Equation 6.4 is then a global minimum. Huber weights (Equation 6.8) satisfy this criterion and so convergence to a stable value is achieved. However, large outliers may still result in a bias in the Huber estimate. A redescending estimator may be employed after the Huber estimate has been found to totally reject any such data. The Huber estimate is a 'better' location to start using a redesending estimator than the ordinary LS estimate and therefore multiple solutions are less likely to occur. This is the approach outlined by Egbert and Booker (1986) and Chave et al. (1987).

The Huber estimate of $\beta_{x}$ and $\beta_{z}$ was then determined in the frequency domain for each storm. It was necessary to rearrange the results of FT to group the fc's according to Fourier frequency. For each storm a file was generated, for every Fourier frequency up to $1 \mathrm{cpd}$, that contained the fc's for all contributing observatories.

The details of the IRLS scheme and parameters used are as follows; the robust measure of scale for $u$ (Equation 6.7) was taken as $\sigma_{r}$ while $k$ of Equation 6.8 was 1.5 as used by Egbert and Booker (1986). The Huber weights were used over 10 iterations which was found to be sufficient for convergence to within 5 decimal places. After this set of iterations the redescending function of Thomson (1977) was used to reject any wild data points. The weight function of Thomson is defined as

$$
w\left(u_{i}\right)=\exp \left\{-\exp \left[k\left(\left|u_{i}\right|-k\right)\right]\right\}
$$

where the parameter $k$ serves a similar purpose to the transition point of the Huber weight in determining when downweighting begins. The value of $k$ was found by evaluating the $N$ th quantile (Section 6.4.4) and is therefore dependent on the sample size $N$, the number of observatories ${ }^{2}$. Therefore the increased probability of outliers in larger datasets is taken account of as $k$ becomes greater to widen the limits of acceptable values. The estimate of scale used in the definition of $u$ was taken as $\sigma_{r}$ as for the

\footnotetext{
'It was intended to use the $N$ th quantile for $k$. However, the 'quantile' determined was the value halfway between the $N-1$ th and $N$ th which results in a slightly smaller and 80 more severe weighting algorithm. It should be noted that the values quoted by Chave et al. (1987) for the $N$ th quantile actually refer to this midway point.
} 
Huber weights. The IRLS procedure was continued for a further 5 iterations with the Thomson weights. Figures 6.3 and 6.4 give examples of the ordinary LS and robust IRLS fit to the observatory Fourier coefficients.

\subsubsection{Assessment}

In a qualitative manner the affect of the robust procedure is shown by the examples of Figures 6.3 and 6.4 . Figure 6.3 refers to storm 7 which is a very clearly defined storm (Figure 2.1) and described by a large number of observatories. It would be expected that if any storm were to provide reliable data this would be one of them. It is apparent that the $P_{1}^{0}$ term is indeed strongly represented in the imaginary parts of the $X$ and $Z$ fc's, diagrams (b) and (d) of Figure 6.3, though it is negligible in the real parts. This is a result of the 'envelope' affecting the fc's which is explained by the time-shift theorem (Section 5.4). The LS and IRLS fit are very similar in the four plots which confirms that for this particular storm the robust method is not of critical importance. The observatory fc's that do diverge from the form indicated by the majority have not biased the LS model significantly, i.e. there are enough 'good' data points for use of the ordinary LS method. It was noted that in general outliers are more common near to the limits of geomagnetic co-latitude.

In contrast to storm 7 the plots of Figure 6.4 refer to storm 1 which is a very energetic and erratic event recorded by only 20 observatories. The fc's are correspondingly of large magnitude and quite scattered. The IRLS method has resulted in an appreciably different model to that of the LS scheme in all four cases. The $P_{1}^{0}$ model of the $X$ fc's, Figure 6.4 (a) and (b), is altered slightly as the 'pull' of the outliers is reduced. In the case of the $Z$ component the estimate of $\beta_{z}$ is very different in both real and imaginary parts. The outliers that exist have a greater affect as they are proportionately more abundant than for storm 7, therefore the IRLS scheme is needed to reduce their influence. The fit to the imaginary $Z$ fc's, diagram (d), is an extreme example as the robust method has changed the sign of the model parameter, the model determined from LS is most definitely inappropriate. Anomalous values near to the ends of the co-latitude range are again mostly responsible for the poor LS fit. Together with Figure 6.3 it appears that observatories near to the extremes of the co-latitude range experience the affects of additional high latitude currents as the measurements do not comply with the simple $P_{1}^{0}$ model source ${ }^{3}$. From this study the chosen limits of $35^{\circ}$ to $145^{\circ}$ geomagnetic co-latitude should be regarded as the maximum limits for such a global analysis, rather than a compromise (Marshall, 1980) which implies the

\footnotetext{
${ }^{3}$ The substorms mentioned in Section 2.1 are caused by these currents.
} 
Figure 6.3. The LS and IRLS determined $P_{1}^{0}$ models for the $X$ and $Z$ Fourier Coefficients. Storm 7, Frequency $0.267 \mathrm{cpd}$.
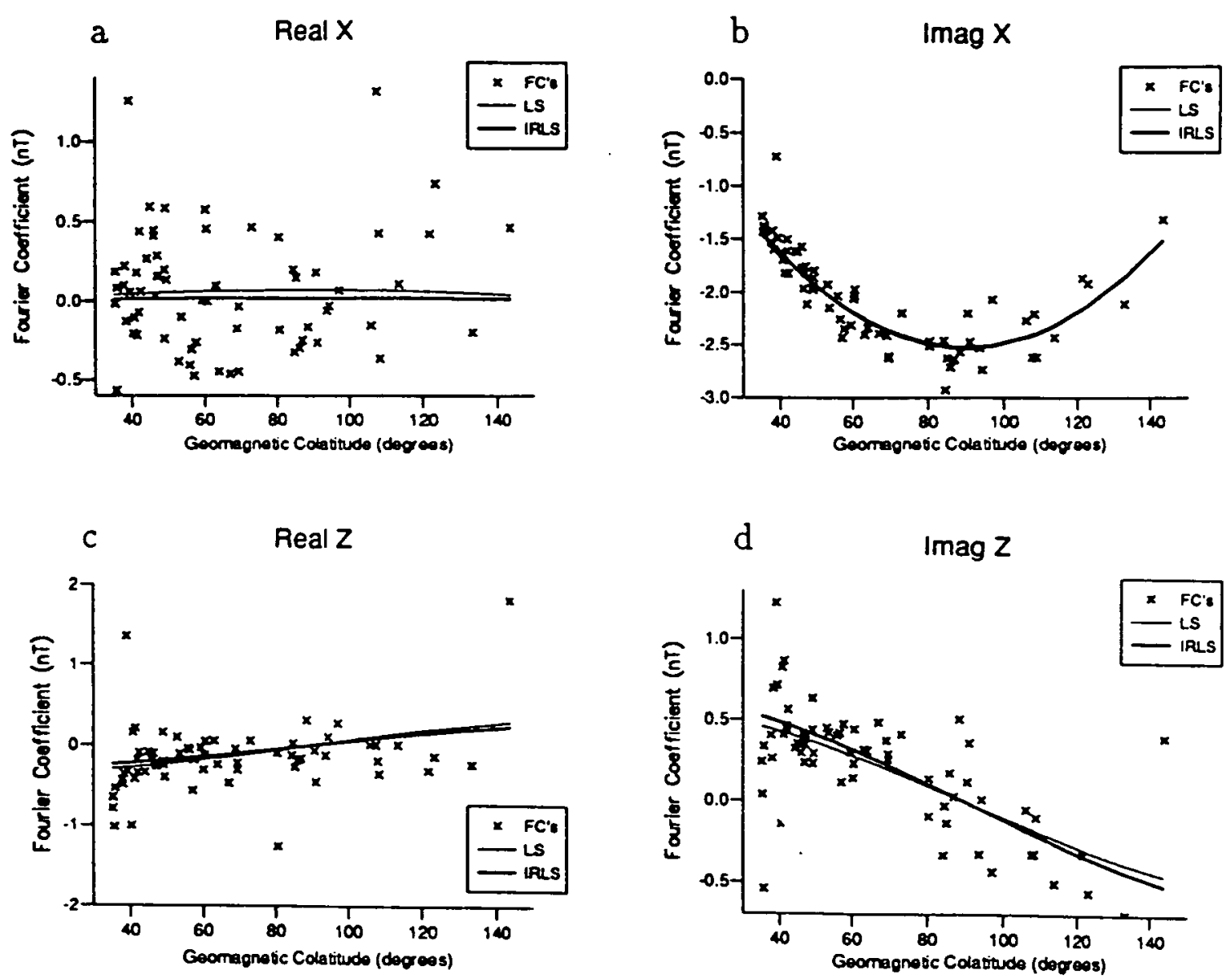
Figure 6.4. The LS and IRLS determined $P_{1}^{0}$ models for the $X$ and $Z$ Fourier Coefficients. Storm 1, Frequency $0.357 \mathrm{cpd}$.
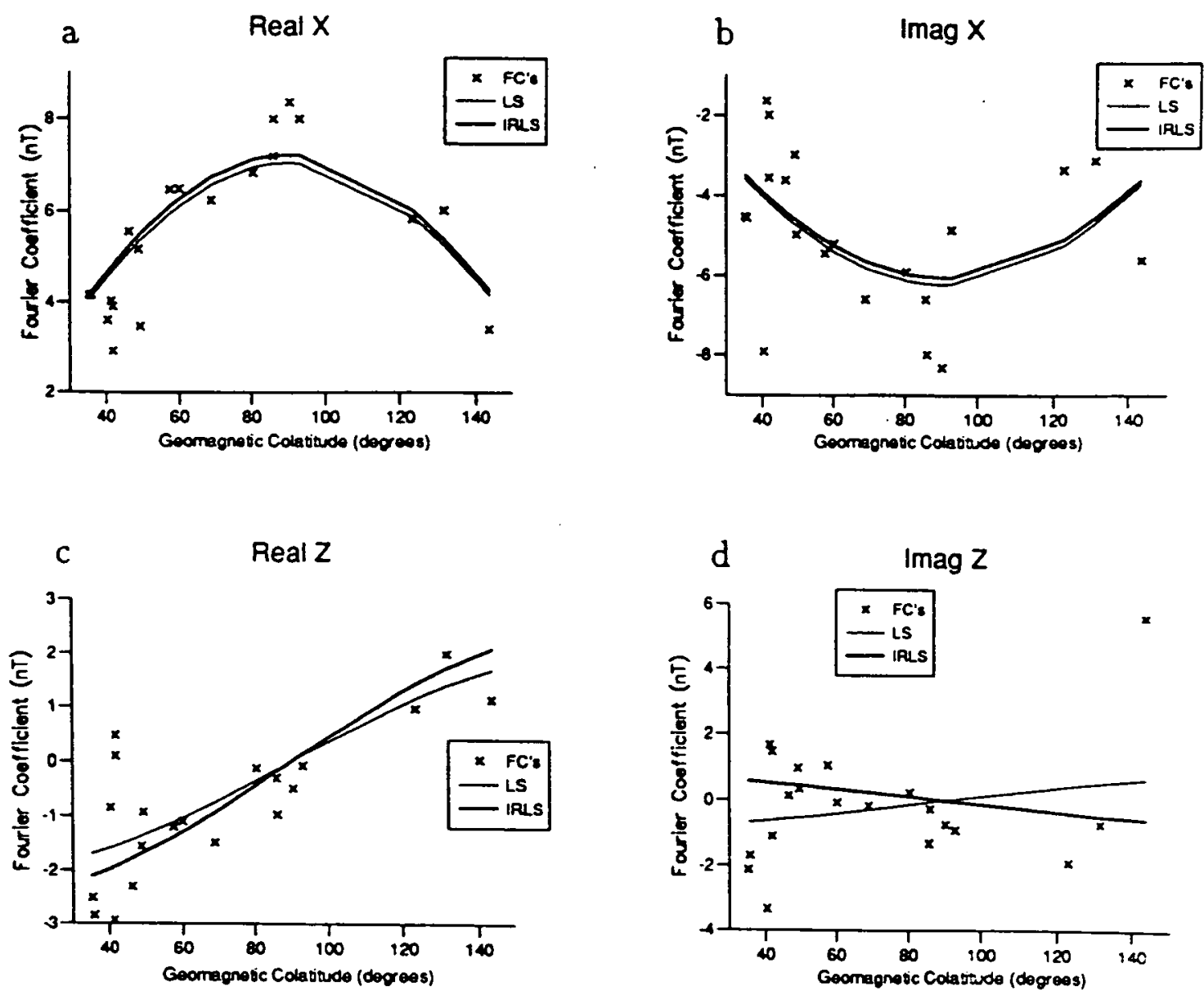
range may be extended.

It is evident that outliers do exist but so far this has only been discussed in terms of observing anomalous data. It is possible to investigate the deviations of the data from the predicted model, ie. the residuals, in a more rigorous manner by comparing their distribution to a theoretical distribution function. This is achieved by the quantilequantile or $Q-Q$ plot.

If some cumulative distribution function (cdf) is denoted by $\Phi$, the $i$ th quantile $Q_{i}$ is given by

$$
Q_{i}=\Phi^{-1}\left(\frac{i-1 / 2}{N}\right)
$$

where $N$ is the number of sampling points of the inverse function $\Phi^{-1}$. Therefore the quantile $Q_{i}$ is the value that occurs with a probability of $(i-1 / 2) / N$. As previously mentioned the distribution underlying the majority of data is assumed to be Gaussian, the value of $Q_{i}$ is then in terms of the standard deviation. When a set of observations is identified with the Gaussian distribution the result is called a normal probability plot.

To constuct a $Q-Q$ plot the residuals $\left[u_{i}\right]_{i=1, \ldots, N}$ are sorted into ascending order so that

$$
u_{(1)} \leq u_{(2)} \leq \cdots \leq u_{(N)}
$$

where $u_{(j)}$ is called the $j$ th order statistic. The order statistics $\left[u_{(j)}\right]_{j=1, \ldots, N}$ are then plotted against the quantiles $\left[Q_{i}\right]_{i=1, \ldots, N}$, this may be thought of as relating the observed to the expected residuals. If the observed residuals match the theoretical distribution the $\mathrm{Q}-\mathrm{Q}$ plot is a straight line passing through the origin with slope 1. Furthermore if two cdf's $F_{X}$ and $F_{Y}$ are related by a linear transformation $Y=a X+b$ the Q-Q plot is the straight line $Q_{i Y}=a Q_{i X}+b$ with slope $a$ and intercept $b$. The slope and intercept give the summary statistics of scale and location for the residual distribution.

Q-Q plots are useful in the detection of outliers which are shown up as extreme points that deviate from the linear relationship, i.e. they are larger than expected. Two papers that discuss $Q-Q$ plots within a geophysical context have been written by Kleiner and Graedel (1980) and Lewis and Fisher (1982).

The interquartile range was mentioned in connection with the fourth spread (Section 6.4.2), it is given by $Q_{3 / 4}-Q_{1 / 4}$ being the range defined by the $25 \%$ and $75 \%$ points of the distribution. It equals 1.349 for the normal distribution.

Normal probability plots were then composed for both the ordinary LS and robust IRLS model fit to the observatory fc's. The LS residuals are purely $\left(Z_{i}-\beta_{z} \cos \theta_{i}^{\prime}\right) / \sigma_{r}$ whereas the IRLS residuals are $w\left(u_{i}\right)\left(Z_{i}-\beta_{z} \cos \theta_{i}^{\prime}\right) / \sigma_{r}$, taking the $Z$ component as the example. The set of weights $\left[w\left(u_{i}\right)\right]_{i=1, \ldots, N}$ are those used in the final iteration. These two groups of residuals were compared to the Gaussian quantiles calculated using the 
function QNTNOR (Ainsworth, 1987).

The plots were calculated for the two examples of model fit previously shown in Figures 6.3 and 6.4, the corresponding normal probability plots of observed residuals against expected are shown in Figures $6.5^{4}$ and 6.6. The distribution of the observed LS residuals is generally heavier tailed than expected in the diagrams of Figures 6.5 and 6.6 which reveals the presence of outliers and desirability of a robust estimation method. The robust (IRLS) residuals are slightly light tailed due to the redescending weighting function having applied low weights to the outliers.

However, within one standard deviation the ordinary and robust normal probability plots of Figure 6.5 (diagrams a,b,c and d) appear to be coincident. Both LS and IRLS residuals closely follow a straight line, slope 1 , and are therefore very nearly normally distributed within the one standard deviation limit. Even outside one standard deviation only a very few LS residuals diverge from their expected value. It was found that the LS and IRLS models of Figure 6.3 were very similar which is explained by the similarity of the LS and IRLS normal probability plots.

The LS and IRLS model fit to the fc's of Storm 1 frequency $0.357 \mathrm{cpd}$ were significantly different (Figure 6.4). The four plots of Figure 6.6 show that the observed residuals of the IRLS fit are of a shallower gradient than for LS within the one standard deviation expected range. This shows that the weighted residuals have been forced into a distribution of a narrower scale and the outliers considerably downweighted producing a light tailed distribution.

The most severely erroneous LS fit is that due to the imaginary $Z$ component fc's, Figure 6.4 diagram (d). The Q-Q plot of this data, diagram (d) of Figure 6.6, shows that the observed IRLS residuals are displaced negatively, in comparison with the observed LS residuals, resulting in a more negative intercept. This indicates a different estimate of location for the residuals corresponding to the appreciable change in the model parameter $\beta_{*}$.

Normal probability plots are then useful to determine what the robust scheme has done in downweighting the residuals. The presence of outliers in the LS case and their reduction after IRLS has been shown. In this assessment of the suitability of the robust method the IRLS residuals are observed to be slightly too severely downweighted, assuming the Gaussian error distribution is applicable. It may therefore be thought necessary to increase the value of $k$ in the Thomson weight (Equation 6.11), however, this approach is based purely on the statistics without considering the physics. It

\footnotetext{
${ }^{4}$ N.B. The two extreme positive observed residuals of diagram (c) fall outside the limits of the plot.

${ }^{3} \mathrm{~A}$ heavy tailed distribution has more values away from the centre than the normal distribution, a light tailed distribution less.
} 
Figure 6.5. Normal Probability Plot for the LS and IRLS fit to the Fourier Coefficients of Storm 7, Frequency $0.267 \mathrm{cpd}$. Expected and observed residuals in terms of standard deviations.
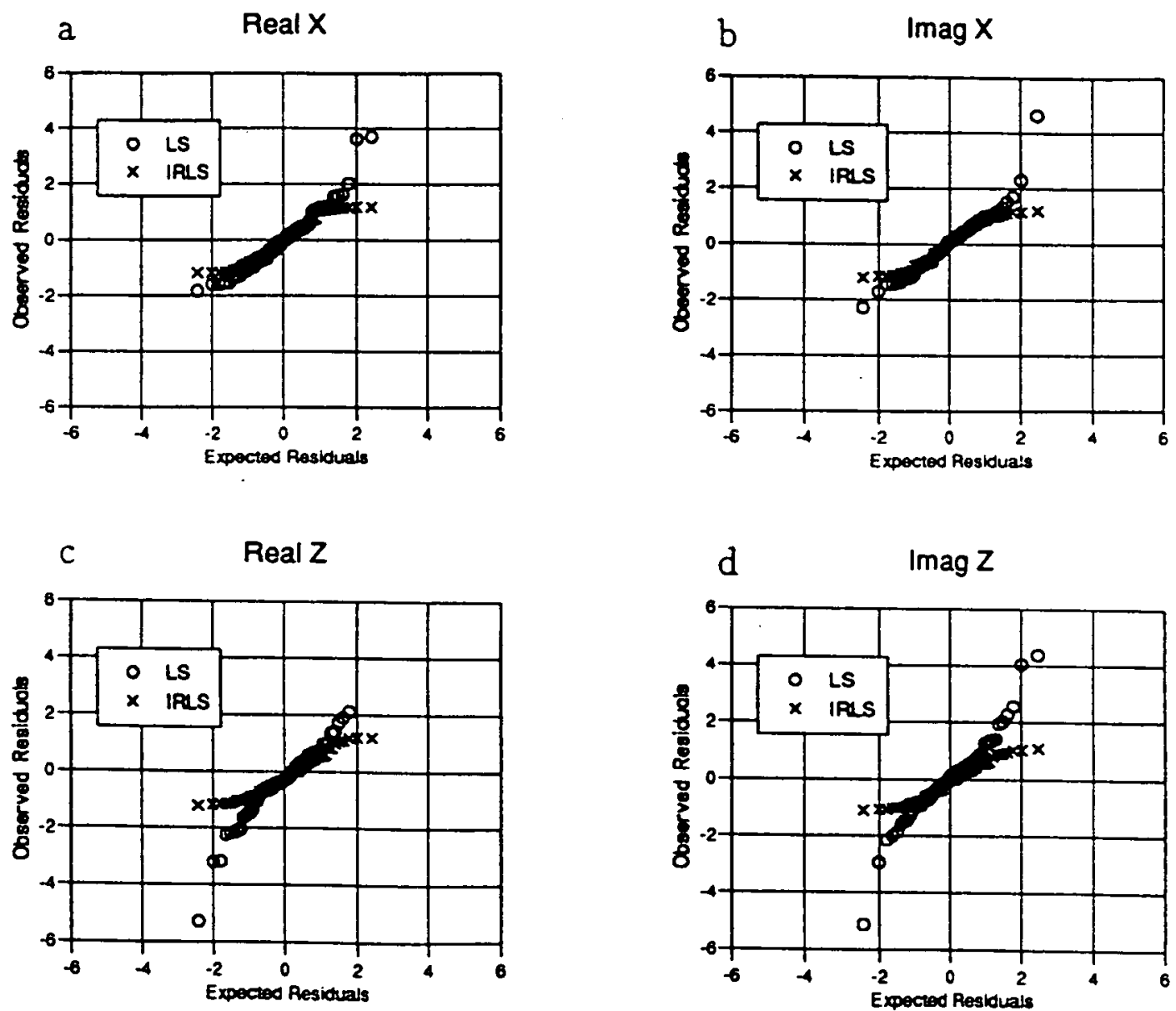
Figure 6.6. Normal Probability Plot for the LS and IRLS fit to the Fourier Coefficients of Storm 1, Frequency $0.357 \mathrm{cpd}$. Expected and observed residuals in terms of standard deviations.
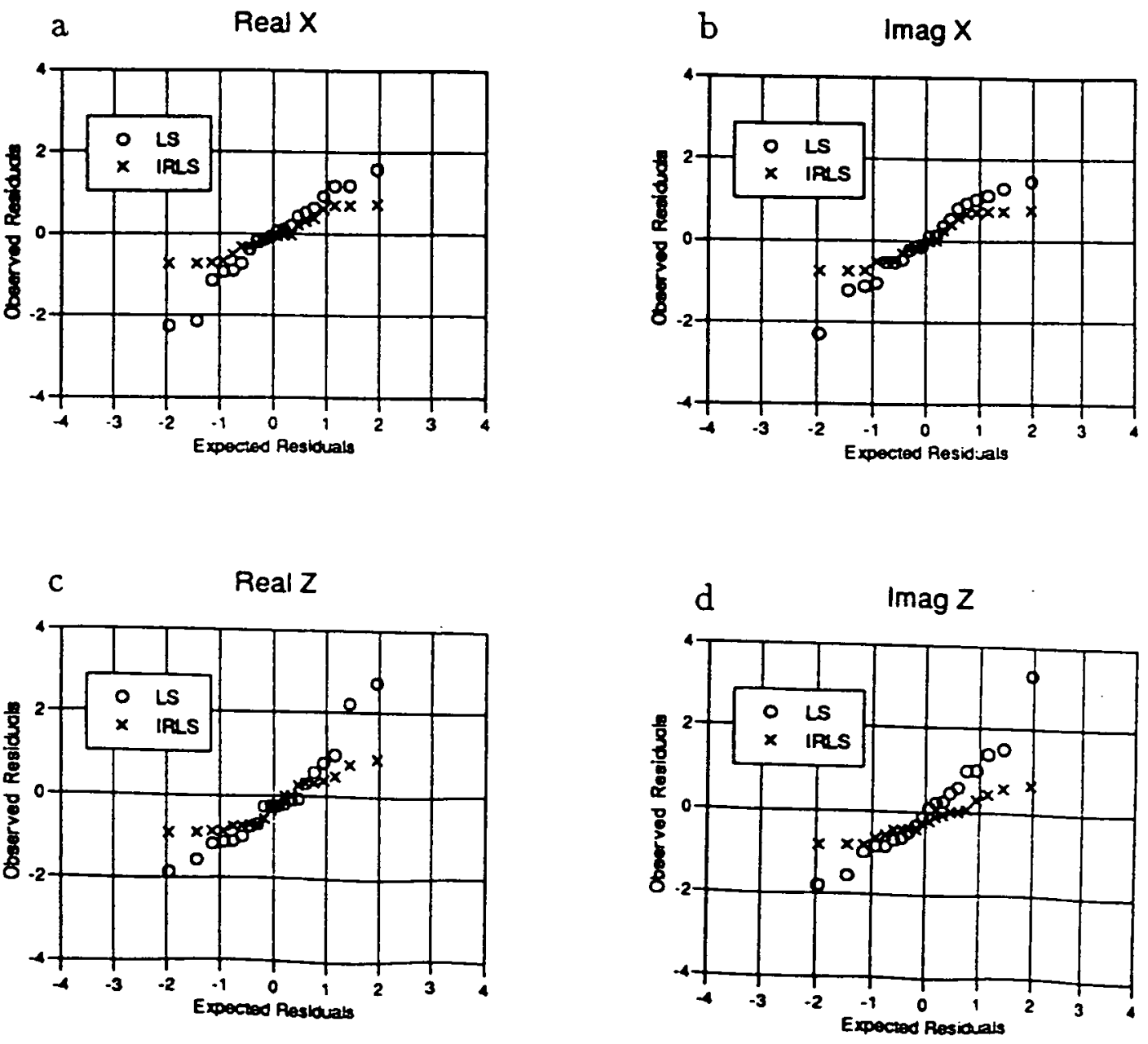
was mentioned above that outliers are more common near to the co-latitude extremes, which is very likely due to complex currents around the magnetic poles. Therefore it is not worthwhile to 'tune' the robust algorithm to achieve the most Gaussian observed residuals when the cause of departure from the model is not solely due to the error distribution. Various examples of Q-Q plots are given by Chave et al. (1987), the robust residuals are also shown to be light tailed. In the case of robust power spectra estimation they state that the light tailed nature does not disrupt the result which can also be said for the presented IRLS derived models of $P_{1}^{0}$.

The robust IRLS method has been shown to be useful in producing a meaningful, unbiased model fit to the observatory fc's. The effects of outliers are diminished while the ordinary LS fit is left unaltered when the data are not dominated by anomalous values. Therefore, as long as the chosen model is present in the data the IRLS technique may be applied with confidence.

\subsection{Separation of Internal and External Parts}

Once the best estimate of $\beta_{x}$ and $\beta_{z}$ in Equations 6.1 and 6.2 had been found robustly it was then possible to determine the separate internal and external parts of potential. From Equations 3.41 and 3.44

$$
\begin{gathered}
\beta_{x}=g_{1}^{0}+g_{1}^{\prime 0}, \\
\beta_{z}=-2 g_{1}^{0}+g_{1}^{\prime 0},
\end{gathered}
$$

the Equations 3.42 and 3.45 disappear for zonal harmonics. If the internal part of $P_{1}^{0}$ $\left(g_{1}^{0}\right)$ is denoted $i$ and the external part $\left(g_{1}^{\prime 0}\right) e$ the Equations 6.12 and 6.13 may be written in matrix form

$$
\left(\begin{array}{cc}
1 & 1 \\
-2 & 1
\end{array}\right)\left(\begin{array}{l}
i \\
e
\end{array}\right)=\left(\begin{array}{c}
\beta_{x} \\
\beta_{z}
\end{array}\right) \text {. }
$$

Solving this matrix equation for $i$ and $e$ leads to

$$
\begin{gathered}
i=\frac{1}{3}\left(\beta_{x}-\beta_{z}\right) \\
e=\frac{1}{3}\left(2 \beta_{x}+\beta_{z}\right) .
\end{gathered}
$$

This operation was carried out on the model parameters determined from the real and imaginary fc's. 


\section{Chapter 7}

\section{Mid-Mantle Global Conductivity}

\subsection{Estimation of Global $P_{1}^{0}$ Response Function}

\subsubsection{Calculation of the Average Response}

The response of the Earth to magnetic variations of different frequencies may be represented by the ratio of internal to external parts of potential. This ratio is called the $Q$ response which is a complex quantity. Therefore once the internal and external parts had been separated (Section 6.5) $Q$ was calculated at the Fourier frequencies $\leq 1 \mathrm{cpd}$ for each storm. The reason why numerous storms had been analysed was to attempt to determine a global $P_{1}^{0}$ response function that would be an accurate representation of the response at mid-mantle depths. An unbiased average estimate of response was therefore required of the combined results from all storms.

The storms extend over different lengths of time and therefore estimates of $Q$ are obtained at different Fourier frequencies. To derive a single average response function the values of $Q$ were grouped according to frequency bands which were defined to be logarithmically equal intervals ( $0.1 \log [$ frequency(cpd)] ). This was done because the skin depth varies as $1 / \sqrt{\omega}$ (Equation 3.21) and so smaller decreases in frequency are required to probe to deeper depths. As the power spectrum decreases with increasing frequency (e.g. Figure 2.2) it is also benefical to have wider frequency bands at the higher frequencies to retain a balance in the distribution of power and enhance confidence in the calculated average by including more values. The effects of a decreasing signal to noise ratio with increasing frequency has been indicated by the FT of the synthetic storm function including added noise (Hobbs, 1987). The derived values of $Q$ from all 44 storms are shown in Figure 7.1, the frequency bands are indicated by the vertical lines.

The entire set of real and imaginary estimates, displayed in Figure 7.1, indicate 
Figure 7.1. The $P_{1}^{0}$ Response Estimates from all 44 Storms. The vertical lines mark the limits of the numbered frequency bands.
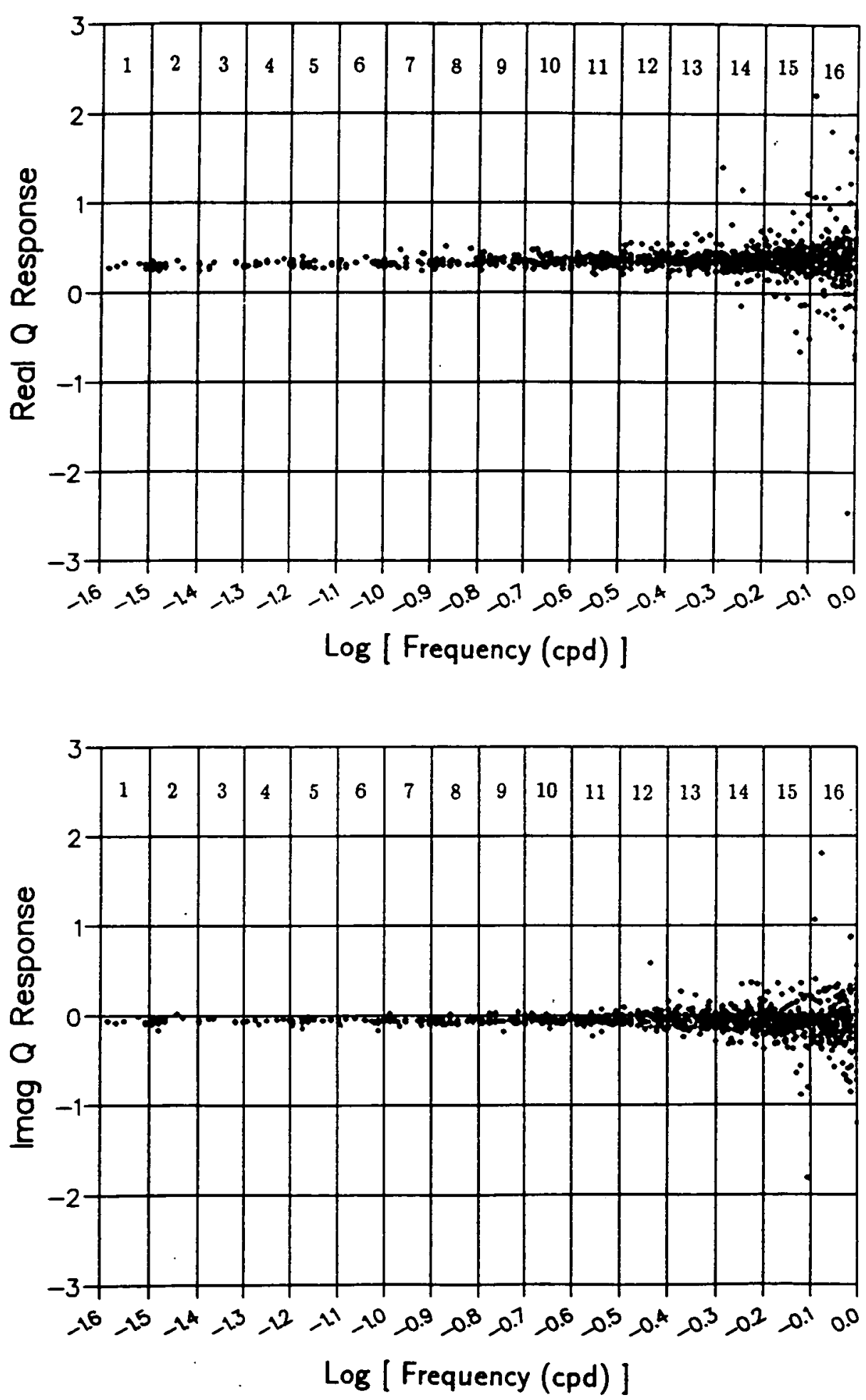
that the $P_{1}^{0}$ response is virtually a linear function for the considered frequency range. The estimates are closely grouped at the lower frequency end and more dispersed for the highest frequencies, however, as there are more estimates for the higher frequency bands this is expected. To determine whether outliers are present normal probability plots were used. This was carried out for the ordinary residual deviations from the mean of each band and for the weighted residuals from the robust average. The plots are given in Figure 7.2 for the real part of $Q$ and Figure 7.3 for the imaginary part, note the change of scale of the vertical axis for the highest bands. Table 7.1 gives the central frequency for each of the sixteen frequency bands.

It is evident that the ordinary observed residuals (LS) form heavy tailed distributions for the highest frequency bands showing that outliers are present. This is probably because the $P_{1}^{0}$ term is not so dominant at the higher frequencies and therefore determination of the coefficient is more variable between different storms, the degradation in accuracy of the fc's with increasing frequency (Hobbs, 1987) may also contribute. Slightly heavy tailed distributions are though also found for the ordinary residuals of some lower frequency bands.

The low slope of the plots indicates that the scale characterising the residuals is smaller than for the expected Gaussian distribution of unit standard deviation. Because the ordinary residuals do not follow a straight line at the higher frequencies the robust IRLS scheme is preferable for use in estimating the location of these bands. The robust method was also used for the other frequency bands to standardise the approach and as the IRLS method does not alter the estimate of location for normally distributed data (Section 6.4.4). However, Table 7.1 shows that differences between the mean and robust average do occur for most bands indicating that the observed data departs slightly from the theoretical distribution.

As the initial step in the IRLS procedure involves ordinary LS and the first few iterations use Huber weights there is an implicit assumption that the distribution of residuals is approximately normal. If the real and imaginary fc's were normally distributed about the $P_{1}^{0}$ term it would be theoretically possible to follow through the steps to complex $Q$ to determine the expected distribution of the response estimates. In this study the more empirical approach of normal probability plots was used to assess whether analysis based on the assumption of normal statistics was valid. Normal probability plots were produced for both the fit of the $P_{1}^{0}$ term to the fc's, Figures 6.5 and 6.6, and the calculation of the average response within frequency bands, Figures 7.2 and 7.3. In both cases the plots revealed that the observed residuals were very close to the expected normal distribution, though ${ }_{\Lambda}$ were some outliers associated with a different distribution. Chave and Thomson (1989) state 'assuming that the Gauss-Markov 
Figure 7.2. Normal Probability Plots of the Residuals for the Real Part of the Response $Q$. Expected and observed residuals in terms of standard deviations.

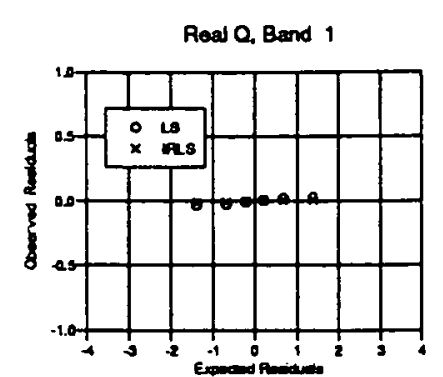

Roal Q, Band 5

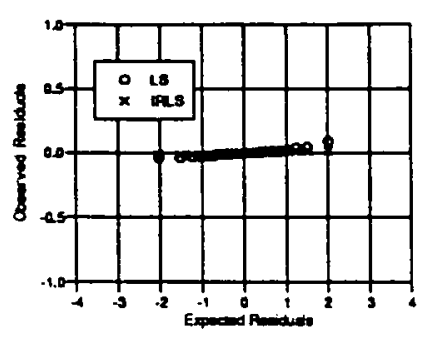

Real Q, Band 8

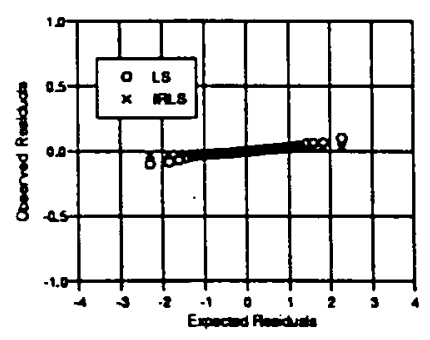

Roal O. Band 13

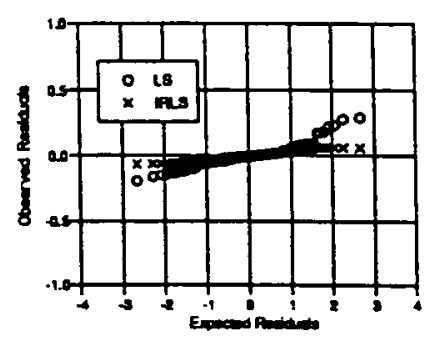

Real Q. Band 2

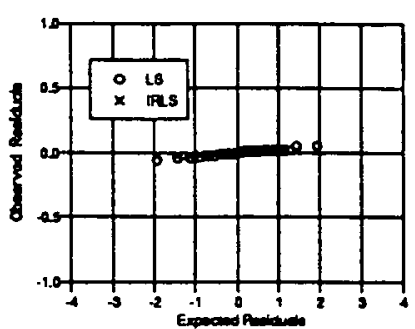

Real Q, Band 6

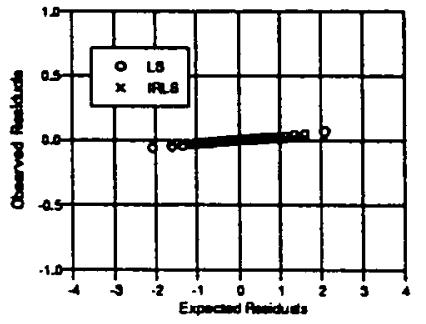

Real O, Band 10

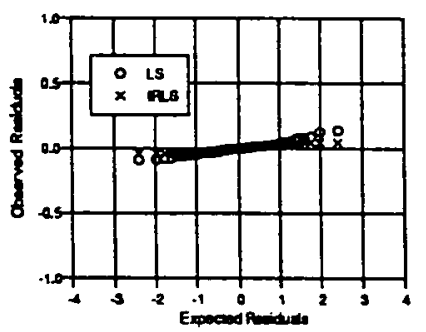

Real Q. Band 14

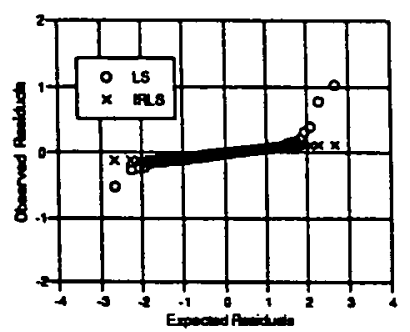

Real Q, Band 3

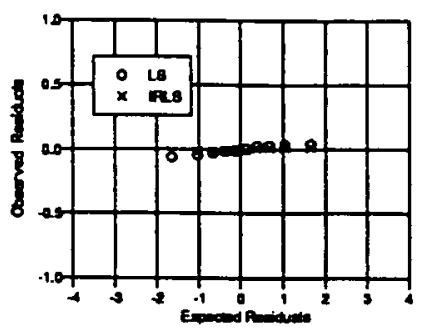

Real Q. Band 7

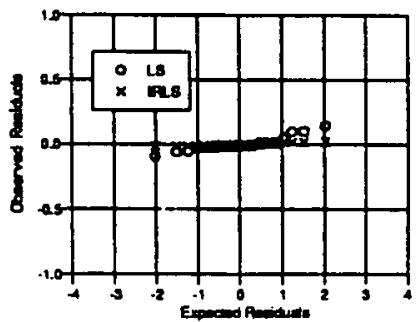

Real O, Band 11

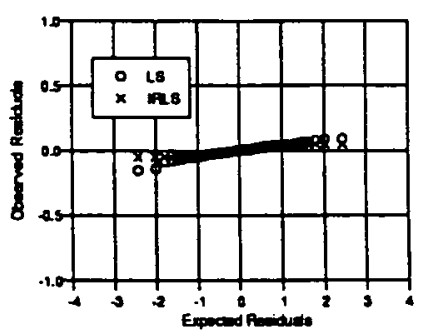

Poal Q, Band 15

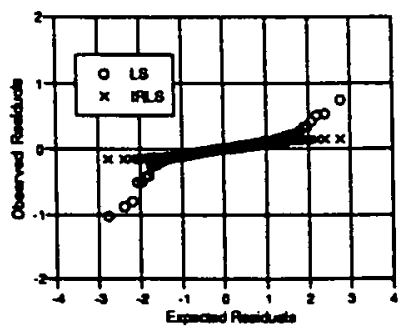

Real 0, Band 4

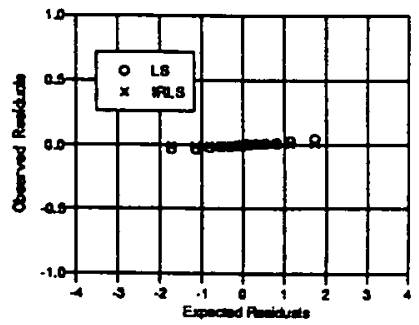

Real O, Band 8

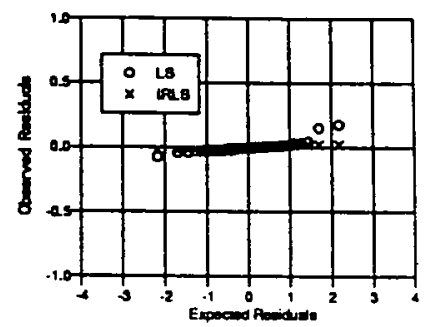

Real O, Band 12

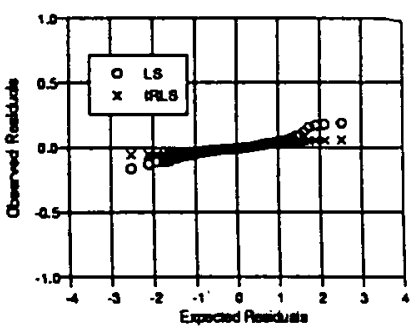

Aoal O. Band 16

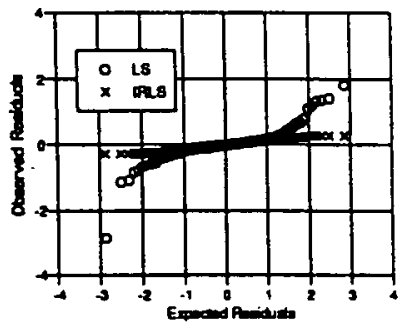


Figure 7.3. Normal Probability Plots of the Residuals for the Imaginary Part of the Response $Q$. Expected and observed residuals in terms of standard deviations.
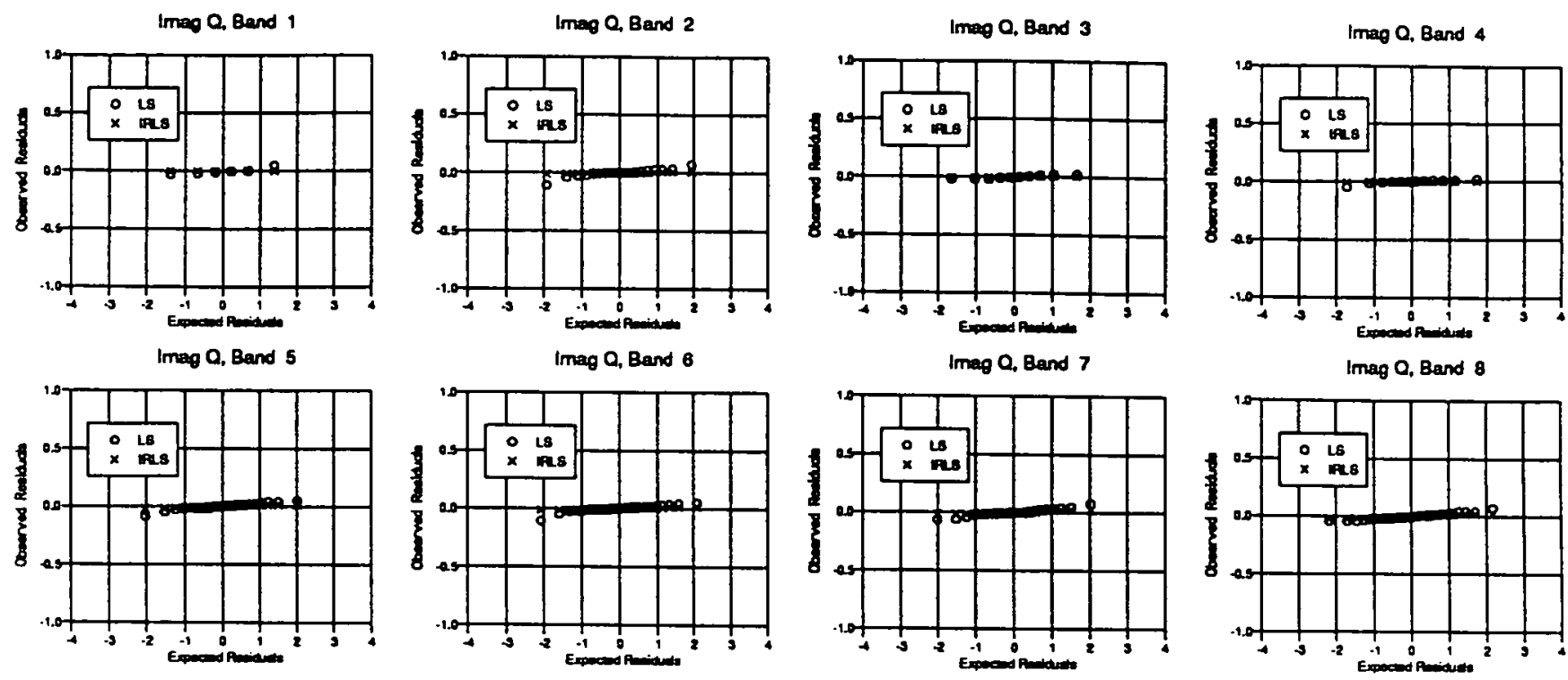

Imag Q, Band 7

Imag O, Band 8
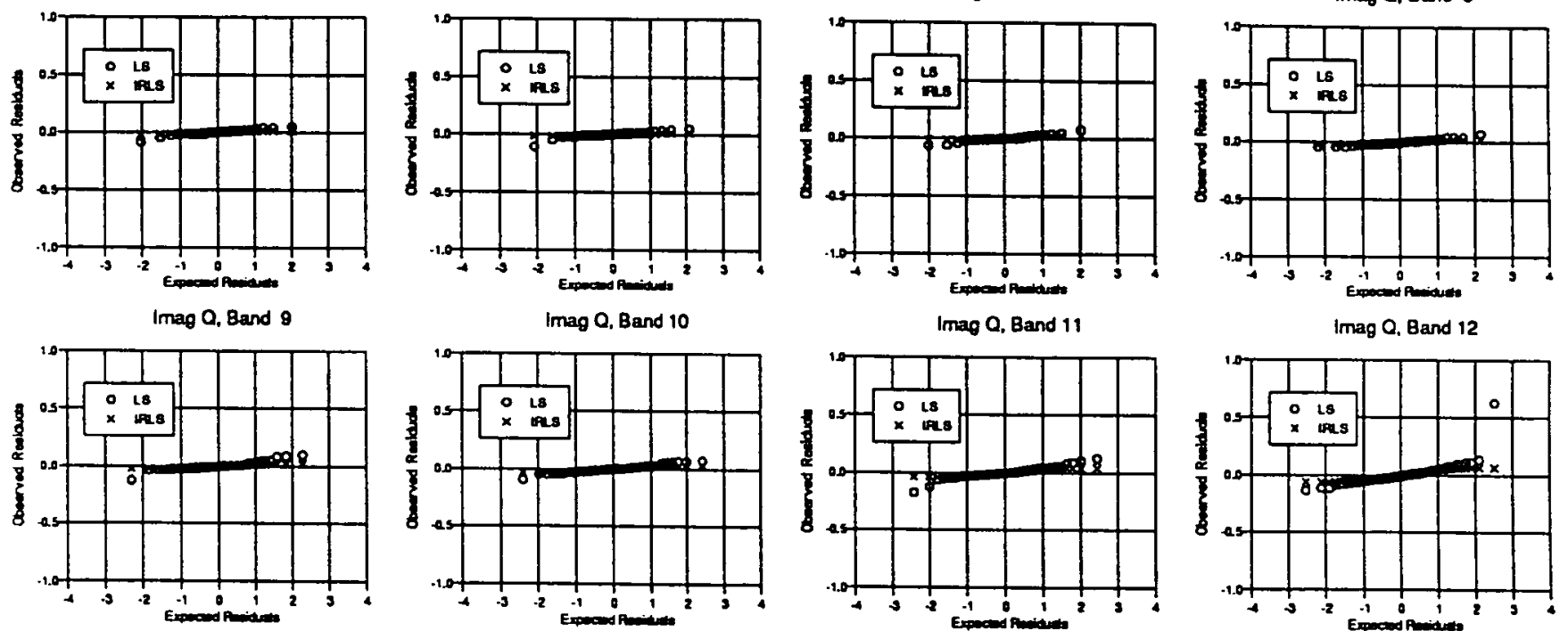

Imag Q. Band 11

Imag Q. Band 12
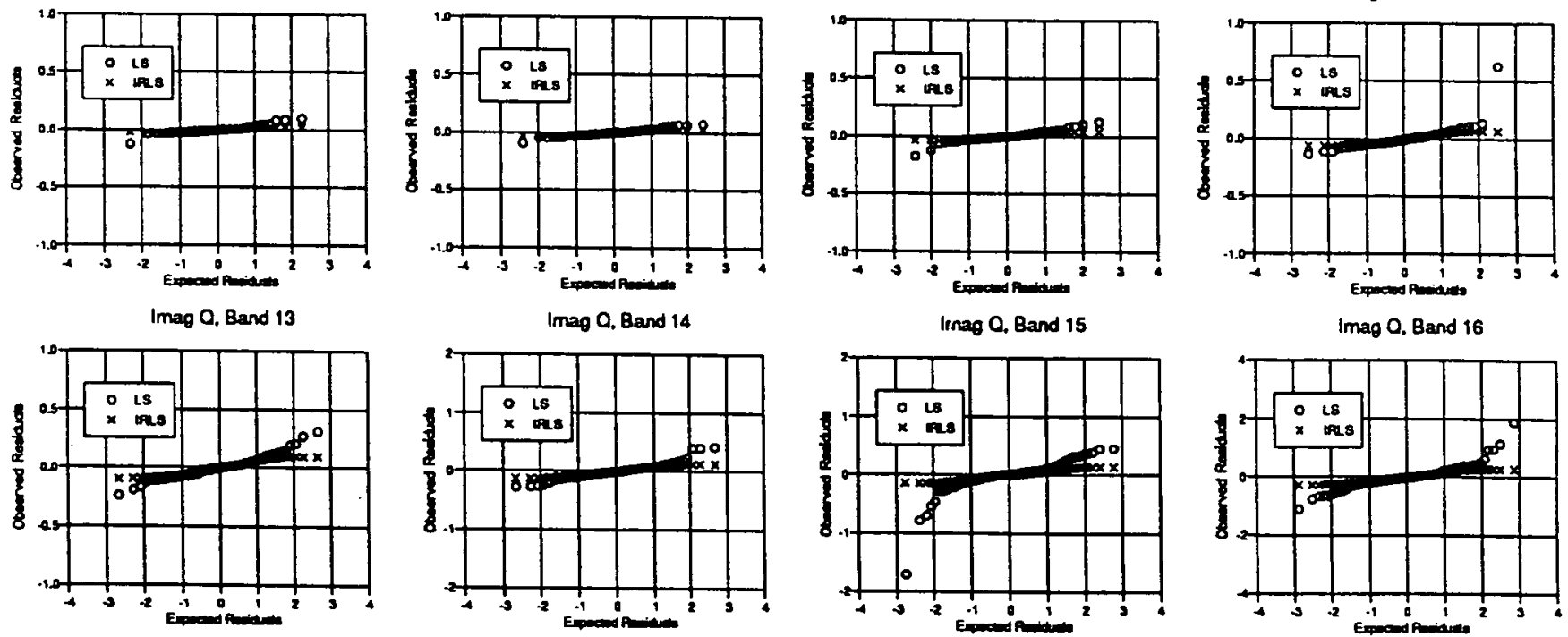
conditions are approximately met, even non-Gaussian frequency domain data can be analyzed with least squares to get reasonably accurate response functions' thus LS based estimators are suitable for near normal distributions. In the light of this statement and the close relationship of the response estimates to the normal distribution, shown by the normal probability plots, the robust IRLS method based on Gaussian statistics was considered valid.

It was necessary to assign errors to the response function based on the spread of estimates. Methods of determining errors that depend on some hypothetical probability distribution are sensitive to outliers. Estimators of scale of this sort are refered to as parametric estimators, the standard deviation of the normal distribution is an example. When the underlying error distribution is not understood and departures from the normal distribution occur nonparametric estimators are more suitable. A simple nonparametric scale estimation method is the jackknife which is explained by Efron and Gong (1983) and has been applied to geophysical data by Chave and Thomson (1989). A brief outline of the technique follows.

Consider a sample of $N$ measurements $x_{1}, x_{2}, \ldots, x_{N}$ which are distributed in an unknown manner. An estimate of the location is denoted $\hat{\theta}$. If one of the measurements $x_{i}$ is deleted the same location estimator may be used to derive a value $\hat{\theta}_{-i}$, the deleteone estimate. The jackknife estimate of standard error is then

$$
s_{J}=\left[\frac{N-1}{N} \sum_{i=1}^{N}\left(\hat{\theta}_{-i}-\bar{\theta}\right)^{2}\right]^{1 / 2}
$$

where $\bar{\theta}=\frac{1}{N} \sum_{i=1}^{N} \hat{\theta}_{-i}$, is the mean of the delete-one estimates. When the estimator is the mean Equation 7.1 gives the standard error $\sigma / \sqrt{N}$. The error estimate $s_{J}$ may be converted to a percentage confidence interval. If the true location of the underlying distribution is $\theta$ then a confidence interval is defined by

$$
\hat{\theta}-t_{\nu}\left(1-\frac{\gamma}{2}\right) s_{J} \leq \theta \leq \hat{\theta}+t_{\nu}\left(1-\frac{\gamma}{2}\right) s_{J}
$$

where $\gamma$ is the probability that the confidence limit is exceeded and $t_{\nu}\left(1-\frac{\gamma}{2}\right)$ is determined from Student's $t$ distribution with $\nu$ degrees of freedom (Chave and Thomson, 1989). The robust estimates of the response for each frequency band, plotted at the central frequency in log space, are shown together with their associated jackknife $95 \%$ confidence intervals in Figure 7.4. The real part of $Q$ reveals a general increase from 0.3 to 0.4 , while the imaginary part is almost constant around -0.05 , exceptions being the first and last two points. The only reason the imaginary part is negative is because of the choice of $\exp [i 2 \pi f t]$ for the FT of the time series data. This conforms to 
Figure 7.4. Robustly Estimated $P_{1}^{0}$ Response $Q$ with Jackknife $95 \%$ Confidence Intervals.

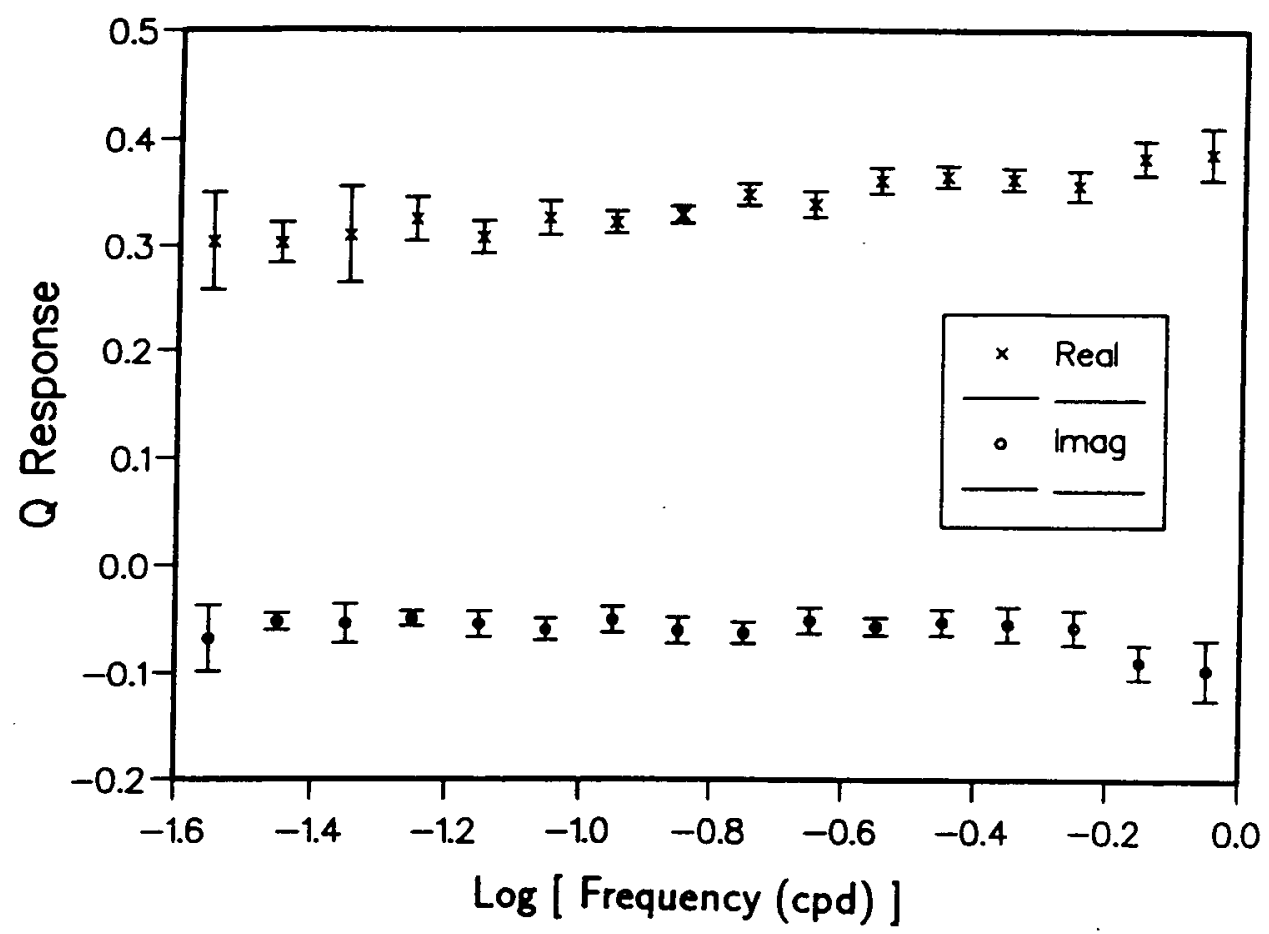

the recommendations of the Semantics Committee (Hobbs, 1992) but for subsequent calculations the sign of the imaginary part was made positive. Thus the phase of $Q$ is then greater than zero and the imaginary part of the $c$ response negative. The derived $P_{1}^{0} Q$ response function is thus physically acceptable. Even when the $95 \%$ confidence intervals are considered the greatest value of $|Q|$ is 0.429 , band 16 , which is below the theoretical limit of 0.5 .

For a comparison the mean and the $95 \%$ confidence intervals derived from the standard error were calculated and are shown next to the robust averages with jackknife confidence intervals in Figure $7.5^{1}$. The ordinary and robust estimates are very similar for all the frequency bands. This agrees with the approximately linear form of the normal probability plots (Figures 7.2 and 7.3) and demonstrates that even when outliers are among the ordinary residuals they are not significant to cause a large bias. The $95 \%$ confidence intervals of the ordinary, mean estimates and the jackknife $95 \%$ intervals

\footnotetext{
'The 'ordinary' estimates are plotted slightly offset for clarity.
} 
of the robust location estimate are of comparable size for most of the bands. The normal probability plots which clearly show the presence of outliers for the real data (Figure 7.2) are bands 13 to 16 for which the jackknife confidence intervals are smaller than their ordinary counterparts (Figure $7.5 \mathrm{a}$ ). The six lowest frequency bands for the real part appear to be approximately normally distributed and in contrast the jackknife intervals are larger than the non-robust error bounds. For the imaginary part of $Q$ the relative size of the errors (Figure $7.5 \mathrm{~b}$ ) would confirm that when the deviations from the mean includes outliers (Figure 7.3, bands 14,15 and 16) the jackknife intervals are smaller than the ordinarily determined errors whereas they are greater when the residual distribution about the mean virtually conforms to normal statistics. Thus the jackknife error estimator is resistant to anomalous values while it assigns conservative errors for near normal residual distributions. For this reason the jackknife errors are preferable. The precise values plotted in the figures discussed above are found in Table 7.1, $\delta$ and $\delta$, are the ordinary and jackknife $95 \%$ confidence intervals.

Table 7.1. The Average $P_{1}^{0}$ Response $Q$ from all 44 Storms.

\begin{tabular}{|c|c|c|c|c|r|r|r|r|r|r|}
\hline \multirow{2}{*}{$\begin{array}{l}\text { Frequency } \\
\text { Band }\end{array}$} & \multicolumn{2}{|c|}{ Band Midpoint } & \multicolumn{4}{|c|}{ Real Part of $Q$} & \multicolumn{3}{|c|}{ Imag Part of $Q$} \\
\cline { 2 - 10 } & cpd & log(cpd) & Mean & $\delta$ & $\begin{array}{r}\text { Robust } \\
\text { Average }\end{array}$ & $\delta J$ & Mean & $\delta$ & $\begin{array}{c}\text { Robust } \\
\text { Average }\end{array}$ & $\delta_{J}$ \\
\hline 1 & 0.028 & -1.550 & 0.302 & 0.028 & 0.304 & 0.046 & -0.061 & 0.028 & -0.068 & 0.031 \\
2 & 0.035 & -1.450 & 0.303 & 0.015 & 0.303 & 0.019 & -0.054 & 0.017 & -0.052 & 0.008 \\
3 & 0.045 & -1.350 & 0.303 & 0.025 & 0.310 & 0.045 & -0.053 & 0.011 & -0.054 & 0.018 \\
4 & 0.056 & -1.250 & 0.328 & 0.015 & 0.325 & 0.020 & -0.054 & 0.011 & -0.049 & 0.007 \\
5 & 0.071 & -1.150 & 0.314 & 0.012 & 0.308 & 0.015 & -0.057 & 0.012 & -0.054 & 0.012 \\
6 & 0.089 & -1.050 & 0.325 & 0.012 & 0.326 & 0.016 & -0.061 & 0.012 & -0.059 & 0.010 \\
7 & 0.112 & -0.950 & 0.336 & 0.023 & 0.322 & 0.010 & -0.052 & 0.015 & -0.050 & 0.012 \\
8 & 0.141 & -0.850 & 0.339 & 0.016 & 0.329 & 0.008 & -0.056 & 0.010 & -0.060 & 0.012 \\
9 & 0.178 & -0.750 & 0.348 & 0.012 & 0.348 & 0.010 & -0.054 & 0.012 & -0.062 & 0.010 \\
10 & 0.224 & -0.650 & 0.344 & 0.012 & 0.339 & 0.012 & -0.048 & 0.008 & -0.051 & 0.012 \\
11 & 0.282 & -0.550 & 0.356 & 0.012 & 0.361 & 0.012 & -0.055 & 0.012 & -0.056 & 0.008 \\
12 & 0.355 & -0.450 & 0.371 & 0.014 & 0.365 & 0.010 & -0.042 & 0.018 & -0.052 & 0.012 \\
13 & 0.447 & -0.350 & 0.368 & 0.014 & 0.363 & 0.010 & -0.047 & 0.014 & -0.054 & 0.016 \\
14 & 0.562 & -0.250 & 0.372 & 0.026 & 0.357 & 0.014 & -0.053 & 0.020 & -0.057 & 0.016 \\
15 & 0.708 & -0.150 & 0.372 & 0.028 & 0.383 & 0.016 & -0.101 & 0.030 & -0.089 & 0.016 \\
16 & 0.891 & -0.050 & 0.403 & 0.049 & 0.387 & 0.024 & -0.090 & 0.035 & -0.096 & 0.028 \\
\hline
\end{tabular}

It was encouraging that the robustly derived estimates of $Q$ for the sixteen frequency bands were physically acceptable and in agreement with past work (Section 7.1.2). It is particularly satisfying that this result was obtained from the 44 chosen storms in a manner that was completely objective. The entire dataset was used and no data was rejected purely on the grounds that it was unphysical. The IRLS scheme did downweight/reject anomalous data according to the adopted weight function and in doing so a realisable solution was obtained though only statistical constraints were 
Figure 7.5. Comparison of the Ordinary Mean and Robust Average.

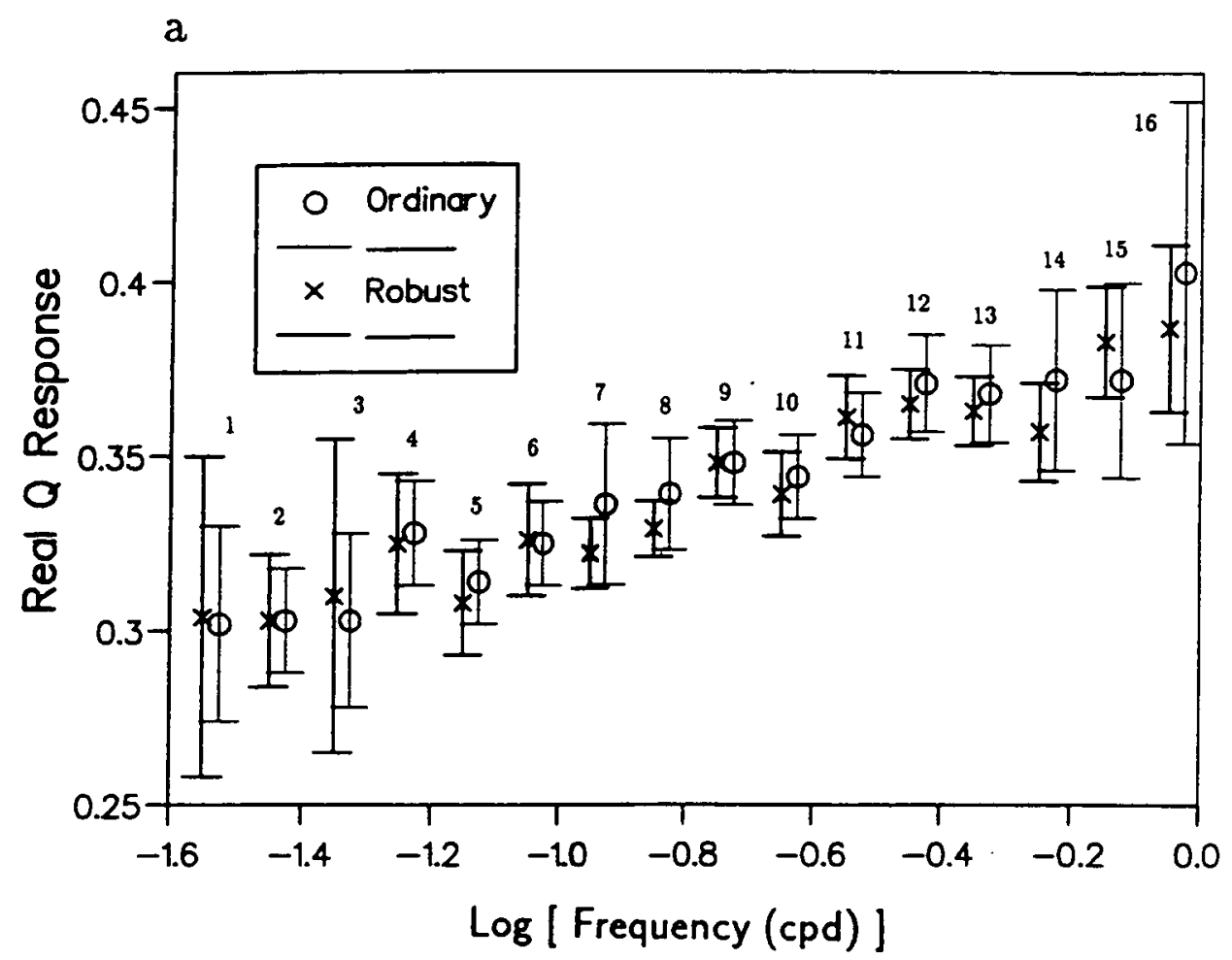

b

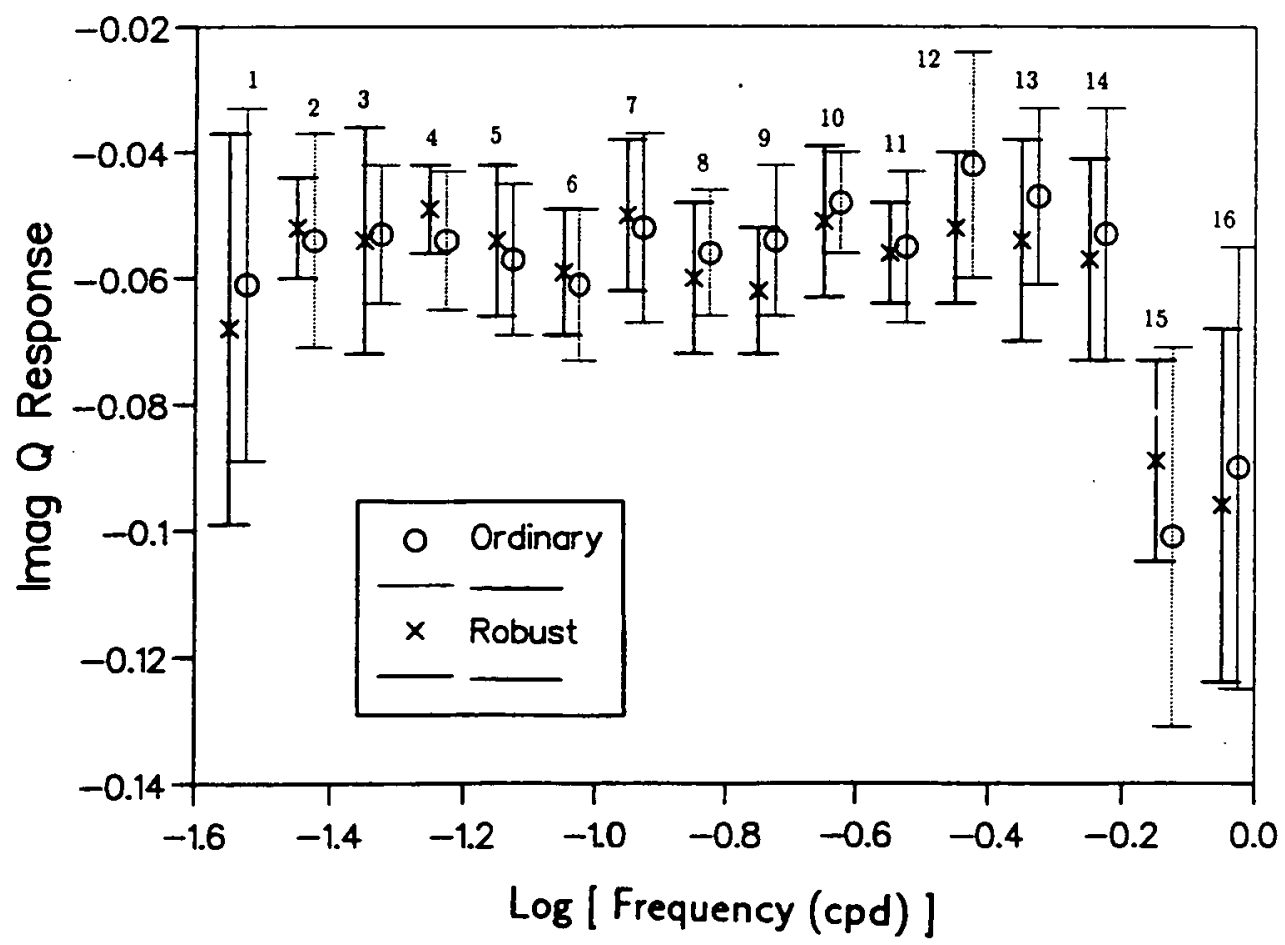


applied. Therefore it was a worthwhile exercise to collate a large storm database to deduce a reliable physical response function. Bassom and Jady (1988) speculated that further storm analyses, to the six of Marshall (1980), would not be profitable - however, the result presented here shows that the effort in treating numerous storms has paid.

The programs for inverting the data require a different form of the response as input. The estimates of $Q$ were then converted to the $c$ response and then to the reponse in terms of apparent resistivity and phase. This was achieved via the following formulas: The $c$ response for a $P_{1}^{0}$ source is given by

$$
c(f)=\frac{a(1-2 Q(f))}{2(1+Q(f))}
$$

where $a=6371 \mathrm{~km}$, the radius of the Earth. The apparent resistivity was then calculated using

$$
\rho_{a}(f)=i \mu \omega c^{2}(f) .
$$

However, the phase assumed by the inversion routines is not that of $\rho_{a}$ but of the impedance $Z\left(Z=E_{X} / H_{Y}\right)$. The phase of impedance $(\phi)$ is found from the phase of $\rho_{a}$ using

$$
\phi=\frac{1}{2}\left(\operatorname{phase}\left(\rho_{a}\right)+\pi / 2\right)
$$

The standard errors on the real and imaginary parts of $Q$ were propagated to give error estimates on amplitude of apparent resistivity $\left(\left|\rho_{a}\right|\right)$ and phase of impedance. This was achieved by transforming complex $Q$ to real and imaginary parts of $\rho_{a}$ for the area defined by the errors on $Q$. By exploring the space limited by the standard errors on $Q$ the largest deviation to the value of $\rho_{a}$ determined from the exact estimate of $Q$ was found. The worst case was found separately for real and imaginary parts so that these errors assigned to apparent resistivity in the complex domain are not exceeded when $Q$ is associated with the given error bounds. To transform to errors in terms of amplitude and phase the real and imaginary errors were firstly used to determine the length of the square with the same area as the rectangle defined by the separate real and imaginary errors. Thus an error estimate $(\delta c)$ common to both real and imaginary parts was found. The uncertainty in the amplitude of apparent resistivity $\left(\left|\delta \rho_{a}\right|\right)$ was then taken to be the modulus of the complex quantity $(\delta c, \delta c)$, while the error in phase $(\delta \phi)$ was calculated as the maximum angle subtended at the origin as $\left|\delta \rho_{a}\right|$ is rotated about the point $\rho_{a}$, i.e. $\delta \phi=\sin ^{-1}\left(\left|\delta \rho_{a}\right| /\left|\rho_{a}\right|\right)$. As mentioned above the phase of impedance and not apparent resistivity is used, however, as this is only a change in rotation the error estimate is not altered. Depending on the relative sizes of $\delta r$ and $\delta i$, the final error space may be quite different to the rectangular region the former errors 
define, this is a difficulty when expressing errors in different co-ordinate systems. The response estimates in terms of $c$, and $\left|\rho_{a}\right|, \phi$ with 'robust' standard errors, derived from the $s_{J}$ of $Q$, are given in Tables A.1 and A.2 of the appendix.

\subsubsection{Comparison with Previous Results}

The response estimates determined in the above section were compared to a selection of the previously calculated response functions. Figure 7.6 gives the $c$ response for 'This Study' together with the results from five other investigations. Apart from the values of Bassom and Jady (1988) the overall trend in the real part of $c$ is similar to that found earlier, the best agreement being with that of Hobbs (1987) and Roberts (1982). The real part of $c$ from 'This Study', Hobbs and Roberts lies between that of Devane (1977) and Banks (1969). On considering the imaginary part of $c$ there is a wider scatter in the displayed values, although the response presented in this work is markedly more constant than for the other datasets. The imaginary estimates determined here show some correlation with the others for frequencies at either end of the frequency range.

\subsection{A Global Conductivity Model}

\subsubsection{Introduction}

The proceeding section derived an average response function which it is hoped is significant in relation to the global conductivity profile of the mid-mantle. It is then the aim of this section to determine the form of the conductivity distribution responsible for the observed response. There are two fundamental schemes by which the problem may be approached. Either a model could be proposed and using the theory of Section 3.1.3, as in Banks (1969), the response could be found and then compared to that observed, adjustments may then be made to the model to bring the model response in closer agreement. An alternative to the formentioned forward modelling technique is that of inversion, in this instance the data is used directly to produce a model. The relationship between the type of model and its response must be known to proceed with inversion, i.e. the forward theory must have been solved. In Section 3.3.1 the LS method was described as an inversion technique. Least Squares is an example of inversion because the coefficients of the chosen model are determined by the observations via the mathematical assumption that the 'best' model is that which minimises the residual sum of squares.

It is possible that if the assumptions leading to the estimated response are not justified there may be no one-dimensional (1-D) conductivity distribution which explains 
Figure 7.6. Comparison of Global $P_{1}^{0}$ Response Estimates
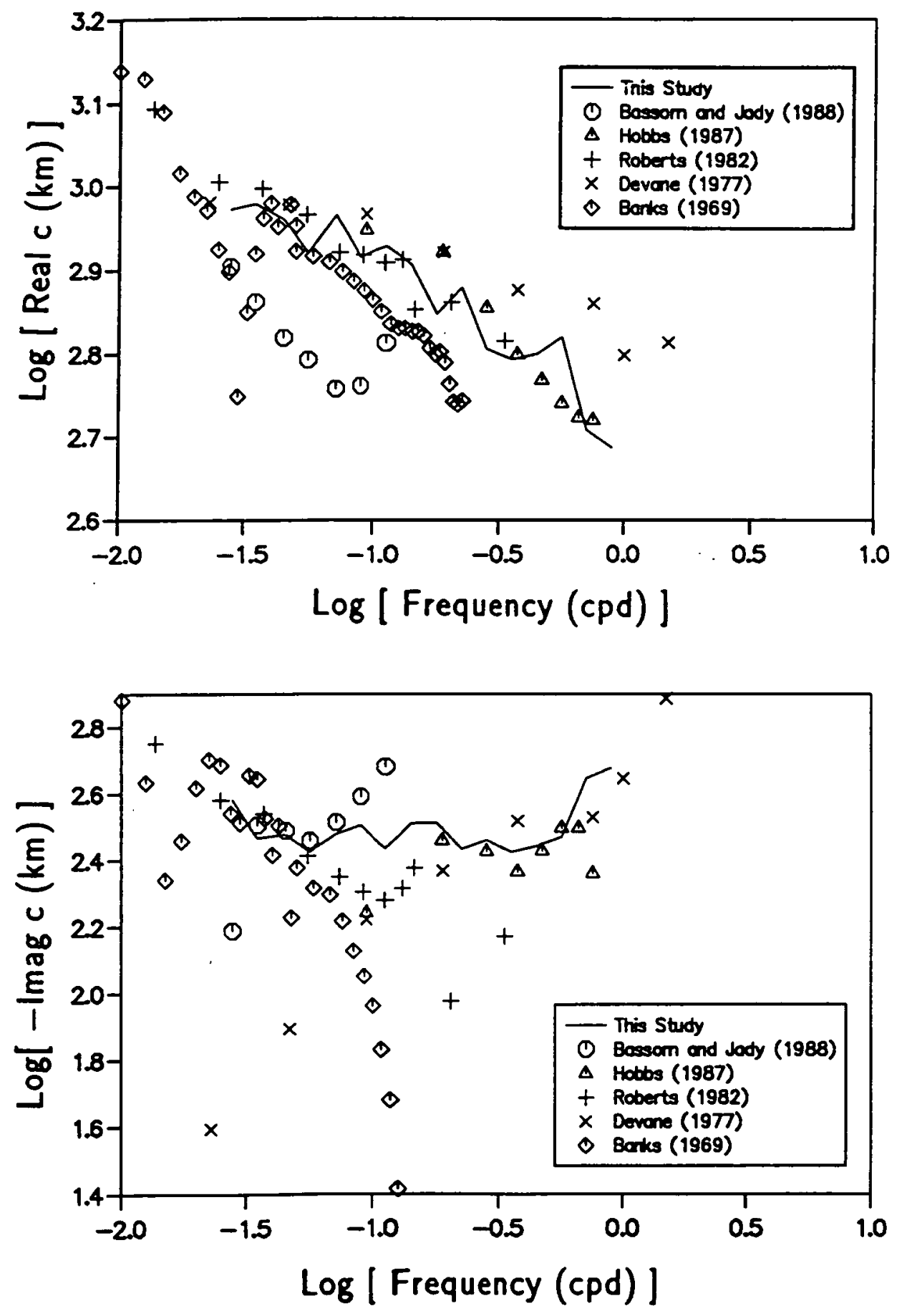
the observations. Thus it is worthwhile to consider the existence of a solution before further modelling is undertaken. This may be investigated using the criteria of Weidelt (1972) or method of Parker (1980). On finding an acceptable model it is, however, inevitable that many other models also exist which 'fit' the data, this is the problem of non-uniqueness. With a finite set of imprecise observations it is not possible to overcome the question of uniqueness, however, some idea of the range of acceptable models may be determined (Parker, 1970). It has been shown (Bailey, 1970) that if it were possible to have exact knowledge of the response from zero to infinite frequency the unique conductivity distribution may be found. This is a useful result implying that theoretically the true structure is obtainable.

A number of inversion schemes have been developed for electromagnetic response data including Parker (1970 and 1980), Hobbs (1982) and Constable et al. (1987). Computer code is available to apply these techniques and it is therefore possible to obtain different types of models and assess their relative merits. These methods are quite varied in the manner they arrive at a conductivity model. In the extreme cases the roughest model that fits the data as well as possible is found by Parker (1980), while the technique of Constable et al. (1987) calculates the smoothest model which fits the data allowing for some chosen level of misfit. The work of the above researchers determines a conductivity structure within a halfspace. However, transformation between a horizontally layered model and a spherically symmetric model is given by Weidelt (1972) and therefore these techniques may be applied to the Earth's global conductivity.

The formulation of the inversion method defines the type of model that is produced, e.g. delta functions within an insulating half space as referred to below, but it is the observed data that determines how the model changes with depth. The work presented in the following section is based on the inversion methods developed by the above workers to derive models of global conductivity. It should, however, be noted that the estimated response assumes the $P_{1}^{0}$ spherical harmonic is a justified respresentation of the source field and that no attempt has been made to account for lateral conductivity variations which exist above $400 \mathrm{~km}$ depth.

\subsubsection{Inversion - Existence of a Solution and Model Determination Existence of a Solution: The $D^{+}$Model}

The primary question to answer on the derivation of a response function is whether or not it is at all possible to model. To resolve this question the response should be tested against a type of model which readily accepts realisable observations. The absolute 
best fit to an EM response function has been found to be given by delta functions of conductivity within an insulating halfspace (Parker, 1980). The mathematical delta function model, denoted $D^{+}$, is not expected to represent the true conductivity structure of the Earth but is fundamental to test the existence of a one-dimensional model. On verification that a $D^{+}$solution fits the data more realistic models may be sought. If however, no $D^{+}$model is found then it is not possible to explain the observations with any 1-D model. The delta function model is based on theory developed by Weidelt (1972). In his paper 22 conditions were given which any continuous response function should satisfy. However, for a finite number of response estimates the $D^{+}$method is the most amenable way of investigating existence and further to Weidelt's criteria $D^{+}$ provides both a necessary and sufficient test (Parker, 1983).

The response function in terms of apparent resistivity and phase (Table A.2) was tested using the $D^{+}$method. The criteria for determining whether the model is a satisfactory fit to the data is the $\chi^{2}$ statistic of the form,

$$
\chi^{2}=\sum_{i=1}^{N}\left(\frac{r_{i}-r\left(\omega_{i}\right)}{\delta_{i}}\right)^{2}
$$

where $N=$ number of estimates,

$r_{i} \quad=$ estimated response,

$r\left(\omega_{i}\right)=$ theoretical response,

and $\delta_{i} \quad=$ standard error, in this study the robust standard error.

There is no precise rule for the $\chi^{2}$ limit defining the boundary between acceptable and unacceptable models. Usually a value somewhat larger than the expected value of $2 N$ is chosen. The expected value is $2 N$ as there are $N$ observations of amplitude and phase or real and imaginary parts. Thus for estimates of the response at 16 frequencies the expected $\chi^{2}$ is 32 , while the expected value plus one standard deviation is 40 . It is therefore necessary for a $D^{+}$model that is unrestricted in depth to better this level of misfit or the response cannot be represented in terms of one dimensional conductivity variations. The $\chi^{2}$ achieved with the apparent resistivity and phase was 7.35 (Figure 7.7) which is well below the test limit and therefore the response may be modelled by a 1-D conductivity model. This is the smallest attainable value of $\chi^{2}$, more realistic models would be expected to have a higher misfit. The $D^{+}$method provides additional information as it also gives the approximate maximum depth within which the conductivity may be modelled (Parker, 1982). If the model includes a perfect conductor then the depth of the model is restricted due to electrical shielding as the skin depth, 
Figure 7.7. The Optimum $D^{+}$Model and Response within an Infinite Insulating Halfspace.
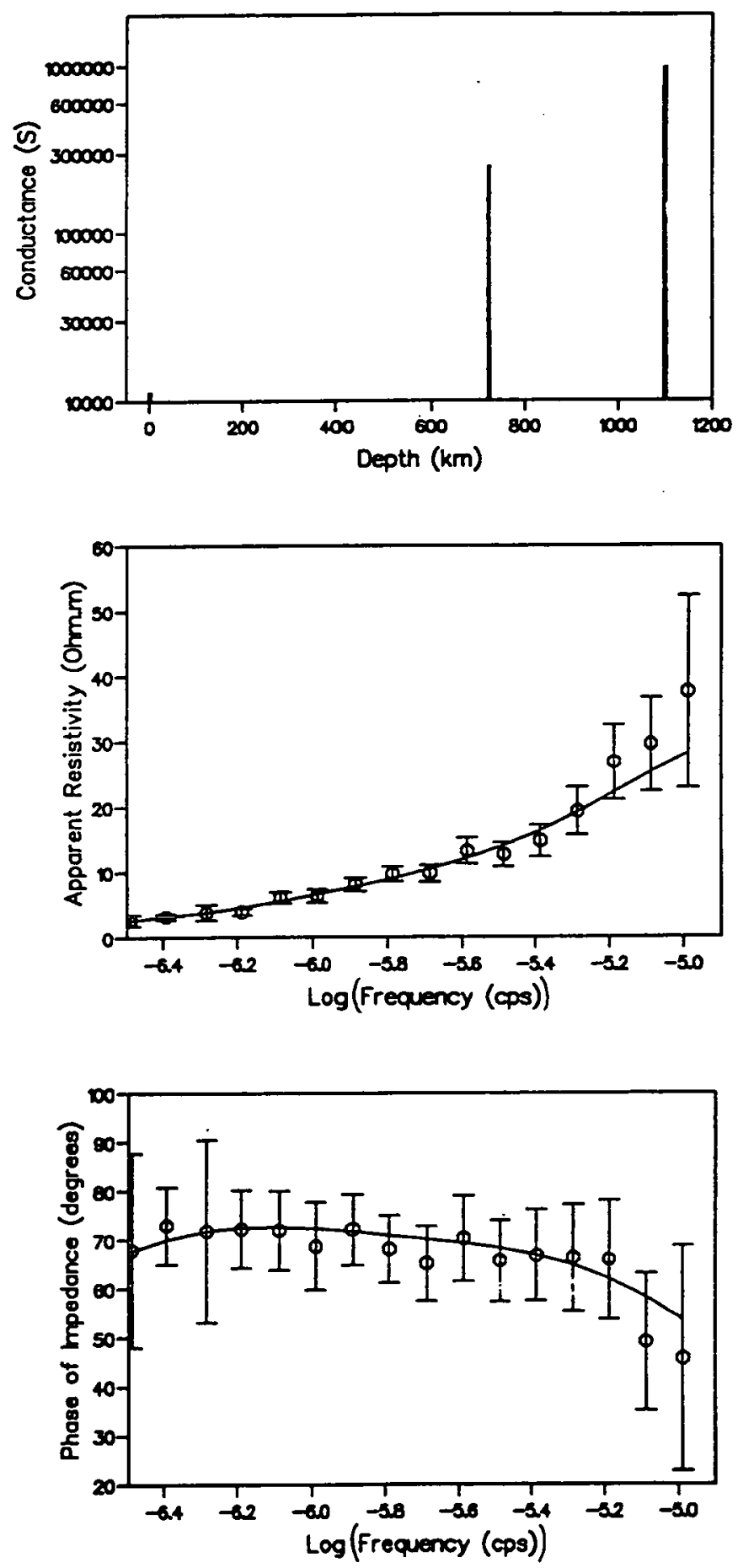
Figure 7.8. The Depth of Penetration of $D^{+}$models against Misfit.

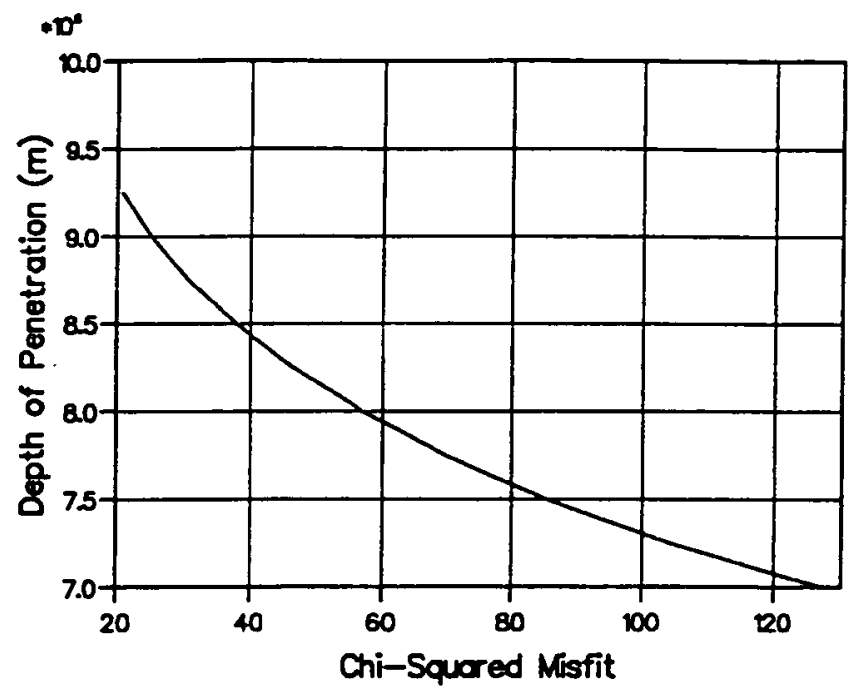

Equation 3.21, is zero at the perfect conductor. The smaller the depth of the perfect conductor the more difficult it is to fit the observed response and so the misfit statistic increases. Taking this the other way round to acheive a particular misfit a certain depth is needed by the $D^{+}$model. It has been stated that a model consisting of delta functions provides the very best $\chi^{2}$ fit to a response function and therefore for some fixed level of misfit other models will require a greater depth. There are an infinite number of other models that have the same misfit but extend to greater depths and therefore the conductivity below this depth is completely arbitrary. If this is the desired level of misfit the depth of the perfect conductor for the $D^{+}$solution may be regarded as the depth of penetration. The plot of Figure 7.8 shows how the depth of penetration relates to the $\chi^{2}$ misfit. Estimation of the depth of penetration by this technique is dependent on the choice of the limit for acceptable models. Taking the sought $\chi^{2}$ to be 40 the depth of penetration for apparent resistivity and phase is approximately 840 $\mathrm{km}$ which is of the expected magnitude. However, as the minimum achievable $\chi^{2}$ is very low the acceptable limit may probably be reduced to at least the expected value of 32 and thus the penetration depth increased slightly ${ }^{2}$.

\footnotetext{
${ }^{2}$ The low $\chi^{2}$ is considered in Section 7.2.5
} 
Figure 7.8. The Misfit $\chi^{2}$ of $H^{+}$Conductivity Models against $d^{2}$.

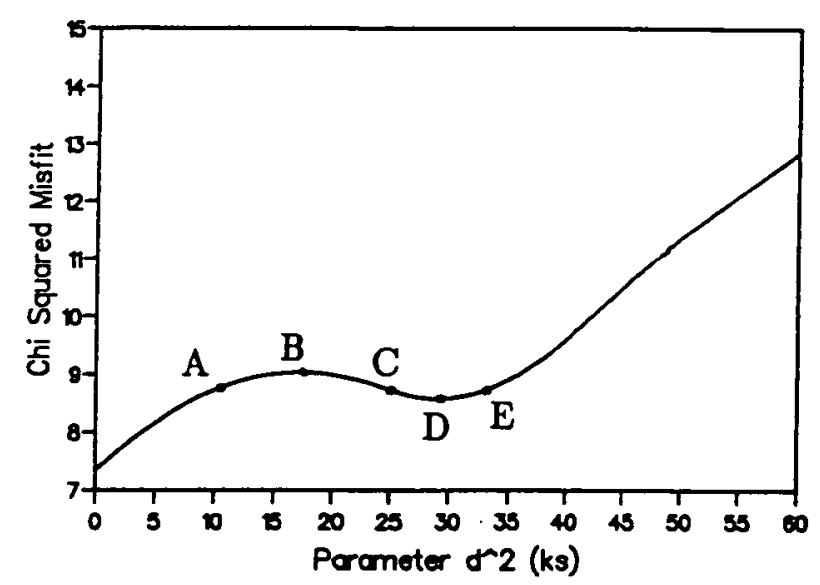

\section{A Layered Model: The $H^{+}$Method}

The theory relating the response to delta functions of conductivity may be extended to thicker layers if the product of layer thickness squared and conductivity is kept constant (Parker, 1980). Models of this type are referred to as $\boldsymbol{H}^{+}$. This constraint is necessary due to the problem of non-uniqueness which restricts the model to be defined in terms of a product of parameters rather than each independently. Thus $H^{+}$models are made up of a stack of layers where the electrical thickness parameter, $d=\sqrt{\sigma_{k} h_{k}^{2} \mu_{0}}$, is held constant throughout all $k$ layers. The parameter $h_{k}$ is the layer thickness. Different values of $d$ may be used to investigate the type of model that fits the observed response. As $d$ tends to zero the $H^{+}$model approaches $D^{+}$and therefore the minimum misfit is approached. However, as the $H^{+}$model is more physically realistic than the $D^{+}$it is of interest to determine $\mathrm{H}^{+}$models that allow a larger misfit to the observed response but are acceptable with respect to the critical value of $\chi^{2}$.

The $H^{+}$inverse method was applied using the apparent resistivity and phase of impedance. Initially the behaviour of the misfit $\chi^{2}$ was observed as $d^{2}$ was varied, this is shown in Figure 7.9. As $d^{2}$ is increased $\chi^{2}$ continues to rise but does not attain the expected value of $\chi^{2}$ within the range of $d^{2}$ permitted by the program. The largest value of $d^{2}$ was $1 \times 10^{5} \mathrm{ks}$ for which $\chi^{2}$ was 30.90 . The $H^{+}$models gave a good fit to the observed data as the low values of $\chi^{2}$ show. The models for the points $\mathbf{A}$ to $\mathrm{E}$ of Figure 7.9 were plotted to view the type of $\mathrm{H}^{+}$solution indicated by the data, 
these are shown by Figure A.10 to Figure A.14 in the appendix. When $d^{2}$ is small the $\mathrm{H}^{+}$solution is made up of thick, nearly insulating layers, and thin highly conducting layers and so approximates $D^{+}$which is difficult to interpret. Increasing $d^{2}$ produces more physically meaningful models though typically 'high' and 'low' conductivity layers alternate which is not expected for the conductivity profile of the mid-mantle. The models for points $\mathbf{C}, \mathbf{D}$ and $\mathbf{E}$ were very similar indicating an increase in conductivity between 700 and $800 \mathrm{~km}$. An interesting result was found on using a large $d^{2}$ of 1000 ks which gave an effective two-layer model with an increase from zero to $0.59 \mathrm{Sm}^{-1}$ at a depth of $530 \mathrm{~km}$, this is presented in Figure 7.10. This model, of $\chi^{2} 25.26$, fits the apparent resistivity well but does not do such a good job in agreeing with the phase.

\section{The Smoothest Model: Occam Inversion}

As the problem of non-uniqueness in deriving geophysical models is unavoidable it seems appropriate to make the model as simple as possible to reduce detail which cannot be confidently included. The idea of keeping solutions simple was encouraged by William of Occam (Russell, 1946; ch. 14) and so the inventors of this inversion scheme (Constable et al., 1987) have taken this name. In this method a 'simple model' is that which is the smoothest acceptable function of conductivity against depth. Models defined in this manner avoid complex solutions which may be over interpreted but indicate that the true conductivity structure is at least as complicated as that given by the model. The propagation of EM fields involves the diffusion of energy, this process is not good at resolving boundaries and therefore suggests the smooth model approach is suitable.

The problem is constucted by defining roughness, the opposite of smoothness, as the integrated square of first or second derivative of conductivity versus depth. It is then attempted to minimise roughness but with the constraint that a certain degree of misfit should be satisfied. For computational purposes the model is specified by a number of layers and so the derivatives are reduced to differences between the layers.

The occam program requires a number of parameters including the total number of layers, the first layer thickness, number of layers per decade, initial half space resistivity, whether a discontinuity is to be allowed and if so its depth, the tolerated misfit, number of iterations and whether the first or second derivative should be minimised. The program was run several times on the derived apparent resistivity and phase to determine suitable values to achieve a model extending to approximately $1500 \mathrm{~km}$ depth. From the $D^{+}$model any structure below $1000 \mathrm{~km}$, though this is being generous with 
Figure 7.10. $H^{+}$model for $d^{2}=1000 \mathrm{ks}$.

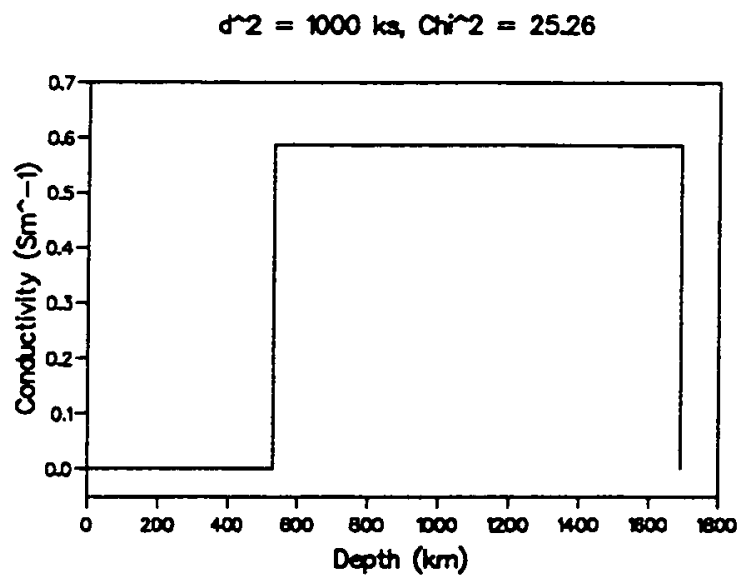

$\sigma_{2}=000 \mathrm{ks}, \mathrm{Cri}^{-2}=25.26$

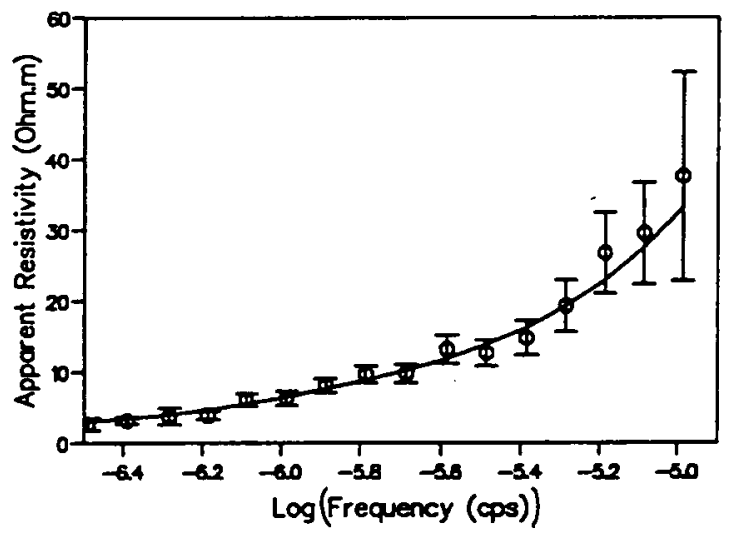

$\sigma^{2} 2=1000 \mathrm{ks}, \mathrm{Chi}^{-2}=2526$

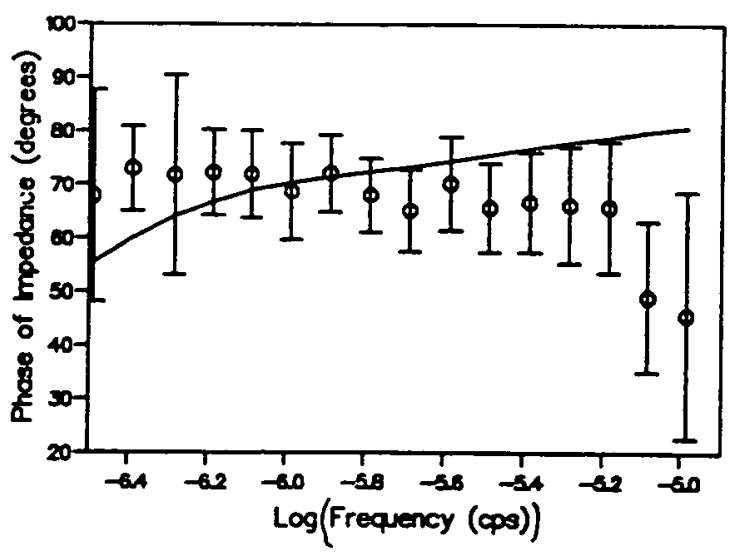


the determined penetration depth ${ }^{3}$, cannot be interpreted with confidence. Different degrees of misfit were then specified and the results of models with and without a discontinuity were assessed when the first derivative and then the second derivative was minimised.

The occam inversion finds the smoothest model which complies with some allowed level of misfit. The tolerated misfit is given as $\sqrt{\chi^{2} / 2 N}$ using the same terms as for $D^{+}$above. Thus assuming Gaussian statistics the expected value of $\chi^{2}$ gives 1 for the tolerated misfit while the best fitting $D^{+}$model with $\chi^{2}=7.35$ would make the absolute minimum tolerated misfit equal to 0.48 .

To begin with models that minimised the first derivative and were continuously smooth were investigated - three such models, labelled A,B and C, are shown in Figures $7.11,7.12$ and 7.13. Model $\mathrm{A}$, with a tolerance of 1 , indicates a steady increase in conductivity to $0.6 \mathrm{Sm}^{-1}$ at about $1000 \mathrm{~km}$, beyond this depth the rate of increase decreases quickly and shows negligible structure. On demanding a better fit to the data, decreasing the tolerated misfit to 0.8 (Model $\mathrm{B}$ ), the conductivity is made lower for the shallowest layers, the increase is more variable, being steepest between 500 and $700 \mathrm{~km}$ and the maximum conductivity reached is greater than for model $\mathrm{A}$ at $1 \mathrm{Sm}^{-1}$. The result of an even stricter fit, misfit restricted to 0.6 , is shown by Model C. The conductivity of the shallowest layers is now similar to that of Model A but the highest value of conductivity, at $1000 \mathrm{~km}$ depth, is greater than both previous models. The gradient of conductivity now takes on a pronounced ' $S$ ' shape with the maximum slope between approximately 600 and $700 \mathrm{~km}$. As the fit to the data is improved the occam aim of minimising smoothness has to be relaxed. It is notable that for the three models the gradient of the theoretical response is less than that of the observed response making the difference between them of opposite sign for each 'half' of the frequency range. Trends such as this in the misfit cannot be removed using the $\chi^{2}$ criterion of goodness of fit.

Using the same parameters as for model A, Figure 7.11, but specifying that the second derivative be minimised the model of Figure 7.14 was found. In this model the rise is of a convex nature, as oppossed to the mainly concave increase of the previous three first derivative models, and the increase in conductivity continues throughout the $1500 \mathrm{~km}$ depth. Similar to model B at $1000 \mathrm{~km}$ the conductivity is $1 \mathrm{Sm}^{-1}$. Defining roughness in terms of the second derivative is a more severe way than using the first derivative and therefore the model shows less variation. The same trend in the misfit,

\footnotetext{
${ }^{3}$ It was discovered that the penetration depth of $840 \mathrm{~km}$ is in fact a conservative estimate, see Section 7.2.5.
} 
as described above, is also present in amplitude of apparent resistivity, while the phase varies quite linearly.

The occam inversion procedure was then allowed to work with a discontinuity at a specified depth minimising the first derivative and setting the tolerated misfit to 1. The program therefore makes the conductivity either side of the discontinuity as smooth as possible but not across the given depth. Three models, E, F and G, are presented in Figures 7.15, 7.16 and 7.17 with the discontinuity at 587, 626 and $668 \mathrm{~km}$ depth respectively ${ }^{4}$. Once a discontinuity is permitted convergence to the acceptable solution is very rapid, considerably more so than for the continuous model. As shown by models $\mathrm{E}, \mathrm{F}$ and $\mathrm{G}$ when a discontinuity is introduced the result is to produce an effective two-layer model.

The occam inversion procedes by iterating from a uniform halfspace to a solution that is the smoothest that satisfies the tolerated misfit. When determining a continuous model the iterative scheme usually surpassed the required misfit and then further iterations were allowed to make the model smoother but with a larger, but tolerated, misfit. However, when a discontinuity is specified no structure other than the step is necessary and the model cannot be made any smoother either side of the discontinuity. Because a smoother model is not possible no more iterations are made to bring the misfit up to the tolerated limit and the program terminated prematurely with a misfit smaller than the tolerated value. Models $F$ and $G$ fit the observed response best, they put a discontinuity between 600 and $700 \mathrm{~km}$ depth, the deeper, Model G, having a higher conducting lower layer.

In Figure 7.18 a discontinuous model derived by minimising the second derivative is shown. The step was specified at the same depth as for model F of Figure 7.16. This model has a higher conductivity shallow layer compared to Model F and does not fit the observed response as well. Unlike the first derivative model the misfit for the final iteration is equal to the tolerated misfit, not smaller, which explains why the fit is poorer although the same tolerance was required.

\subsubsection{Forward Modelling}

Once a type of model is found which seems to fit the observed response, forward modelling may then be used to tailor the model to fit the data more closely. From the above work with occam inversion it appears that a simple two-layer model fits the data well. Forward modelling initially using a two-layer model based on Models F and G

\footnotetext{
'These depths seem quite arbitrary. They are the result of the layering scheme used by the occam program and are the closest layer boundaries to 550,600 and $650 \mathrm{~km}$.
} 
was then pursued using a program written by B.A. Hobbs and G. Dawes.

Different models were tried in an effort to make the observed and model response agree. Trends in the misfit of apparent resistivity were reduced by increasing or decreasing the conductivity of the upper and lower layers and varying the transition depth. The misfit to the phase was considered as different models were investigated so that only those with a reasonable fit to both amplitude and phase were kept. Two models which were found to have a response compatible with the observed response are shown in Figures 7.19 and 7.20. Although the investigation was not extensive no model having a discontinuity at $500 \mathrm{~km}$ was found to fit the observations. In contrast when the transition was made deeper than that of the two discontinuous occam models of $F$ and $G$ a satisfactory result was readily determined (Model J, Figure 7.20).

Both models fit the observed apparent resistivity well but do not do such a good job with the phase. Models I and J show larger values of phase than that observed, though for the most part within the error bounds. The phase of the two models at the short periods is noticably different with the deeper discontinuity Model $\mathrm{J}$ producing a fall in phase of impedance which is indicated by the two shortest period estimates. These two values do not appear to follow the trend set by the majority and are at the extreme end of the useful frequency range, assuming the dominance of a $P_{1}^{0}$ source field (Section 6.3), and therefore their validity is not certain. The problem of non-uniqueness is illustrated in that both these and other similar two-layer models fit the data well. The forward modelling has therefore shown that a two-layer model does fit the observed response but that narrow contraints cannot be applied to the model parameters.

\subsubsection{Inversion - Inference}

The problem of non-uniqueness has been repeatedly referred to in the preceeding sections. As this is such a restriction of geophysical models it is desirable to obtain some information regarding what may be inferred from the model, i.e. determine the significance of the model features. Using their inversion theory Backus and Gilbert (1968) brought the idea of resolution to geophysical models. The method allows an estimate of the uncertainty of a model to be derived in a systematic way, the alternative being to search for acceptable models randomly which is the Monte Carlo method.

The inversion method of Backus and Gilbert was applied to the problem of electromagnetic induction by Parker (1970), he also outlined the methodology giving the resolving power of the model. The method assumes the observed response is sufficiently close to the starting model that the problem may be linearized. This is not a strictly valid assumption to make (Parker, 1983) and therefore the technique cannot completely 
resolve the question of the essential features of conductivity. However, the method does provide a guide to the resolvable features and has been used by previous workers such as Hobbs (1983). The conductivity of the found model at depth $z_{0}$ is thought of as being a smoothed estimate of the true structure near to $z_{0}$. The smoothed averaged conductivity $\left\langle\sigma\left(r_{0}\right)\right\rangle$, i.e. the model estimate of conductivity, may then be written

$$
\left\langle\sigma\left(r_{0}\right)\right\rangle=\int_{0}^{a} \tilde{\delta}\left(z, z_{0}\right) \sigma(z) d z,
$$

where $\sigma(z)$ is the true conductivity variation and $\tilde{\delta}\left(z, z_{0}\right)$ is a function of $\operatorname{depth}(z)$ having its maximum at $z_{0}$ and is small elsewhere, its width is approximately equal to the resolution. It is the objective to find the optimal resolution function $\delta\left(z, z_{0}\right)$ indicating how well $\sigma(z)$ is understood, this is carried out by minimising the spread of the resolution function. The spread is defined so that small values correspond to good resolution, meaning the resolution function has a narrow peak. There is an inverse relationship between spread and precision which is dependent on the model and observed errors and also varies with depth. Thus for a particular model and depth the smaller the required uncertainty in conductivity the larger the spread and so poorer resolution. This may be shown by a trade-off diagram which gives the spread, normalised by the depth of the model, plotted against depth and relative error $\Delta \sigma / \sigma$. A model average plot may be made from the trade-off diagram by choosing a certain level of relative error and plotting two dimensional error bars using the relative error in conductivity and associated normalised spread in depth drawn about the model averages.

The above Backus-Gilbert method of determining resolution was applied to a twolayer model with a discontinuity at $680 \mathrm{~km}$, upper layer of conductivity $0.03 \mathrm{Sm}^{-1}$ and lower layer $1.43 \mathrm{Sm}^{-1}$, in terms of resistivity $33.2 \Omega \mathrm{m}$ and $0.7 \Omega \mathrm{m}$ respectively. This is a suitable model as during the forward modelling it was found to fit the observed response well and is an intermediate case as the discontinuity is between the models of Figures 7.19 and 7.20. The model and its response are shown in Figure 7.21. This figure shows resistivity rather than conductivity, as used in previous diagrams, and apologies are made for this change. The trade-off diagram together with model average for relative error of 0.3 are displayed in Figure 7.22. The trade-off diagram reveals that the best resolution is found below the discontinuity and that it is possible to determine the conductivity to a spread of $0.2(200 \mathrm{~km})$ with relative error of 0.2 down to approximately $800 \mathrm{~km}$. As the conductivity is constant for each layer the spread is large above the layer boundary as the depth range averaged over to give the conductivity estimate may include the whole layer. The only feature of this model is the discontinuity 
and therefore what is required from the trade-off and model average plot is whether the jump in conductivity is essential model structure or not. From the model average, which only shows the better estimates of spread, a discontinuous model is not the only model indicated as a smoother decreasing function of resistivity would be possible within the limits of uncertainty. This is not surprising considering that the occam inversion showed that there are smooth models which adequately fit the data.

As the relationship between spread and relative error is dependent on the model the Backus-Gilbert technique of estimating inference was also carried out using a smooth model for comparison. For consistency it would have been best to determine the resolution for a smooth occam model but transforming it to the particular depth scale required by the program (Hobbs, 1983) was not straightforward - an alternative 'smooth model' was generated using the method given in the forementioned paper and is shown in Figure 7.23. The corresponding trade-off diagram and model average are given in Figure 7.24. For this model the trade-off diagram contours of spread show that the resolution improves down to $900 \mathrm{~km}$ and degrades thereafter. The region enclosed by the 0.2 contour is more restrictive in relative error than for the two-layer model.

This investigation demonstrates how the resolution is dependent on the model. Both these models, Figures 7.21 and 7.23, have been derived from the same observed response and it is found that the variation of spread with depth is related to the 'amount' of structure, or gradient of the model at that depth. Thus at the depth of the discontinuity of the model in Figure 7.21 where the gradient in conductivity is infinite, the spread is the smallest of the two models. In contrast approximately half way into the upper layer of the discontinuous model, around $350 \mathrm{~km}$, the spread obtains a maximum. This is the point where the conductivity may be found by averaging over the largest range in depth. The trade-off diagram for the smooth model, Figure 7.24, shows a smaller spectrum of spread as the gradient in conductivity is finite everywhere and the upper region of constant conductivity is shallower.

This attempt to determine how well the data resolves the conductivity distribution has not indicated a well defined model as many functions satisfy the model average plots. The types of models used do not appear well suited to the method due to the regions of constant conductivity and also the linearization of the technique may be a factor hindering the result. The method also depends on the errors associated with the response which are discussed in the following section and may have contributed the magnitude of spread. 


\subsubsection{Interpretation}

In this chapter various techniques have been employed to investigate the conductivity of the mid-mantle implied from the derived response function. The response determined in Section 7.1.1 was used on its own without including any previous results. Other response estimates were not incorporated as it was thought better to see what information regarding the problem of global conductivity was implied by this dataset alone. In Section 7.1.2 the agreement between the response found in this study and that of past work was shown to be quite good considering the different approaches taken. However, the correlation is not sufficient to directly combine the datasets and most importantly the way in which the errors were estimated is not compatible. The presented models were derived for a plane rather than spherical Earth and it was mentioned in Section 7.2.1 that a transformation exists to convert between the two. However, for the depth extent of the models the effect of the transformation is very small and it is therefore not necessary.

The inversion and forward modelling proved to readily find 1-D models that produced a very good $\chi^{2}$ fit to the data. The $\chi^{2}$ obtained from the $D^{+}$and $H^{+}$inversion, Figures 7.8 and 7.9 is very low and in the case of $H^{+}$it was not possible to attain the expected misfit within the limits of the program. In comparison with the plot of $\chi^{2}$ against $d^{2}$ of Parker and Whaler (1981; Figure 4) it is notable that the diagram of Figure 7.9 gives much smaller misfit values. The reason for the low $\chi^{2}$ obtained in this study was therefore investigated.

Although the determined response in terms of apparent resistivity and phase of impedance was used as input to the two forementioned inversion schemes the data was converted to the complex $c$ response within the computer code and so the errors were also transformed. Thus from estimating the response $Q$ transformations have been made to $c$, then to $\rho_{a}$ and $\phi$ and then back to $c$. This is not ideal for retaining the precision of the originally calculated errors as at every change in form of response the poorest transformed estimate of error is kept as a conservative measure. The conversion of errors on a complex quantity defined by real and imaginary parts to some other in terms of amplitude and phase is especially troublesome. The $D^{+}$program displayed the $c$ response calculated from $\rho_{a}$ and $\phi$ with transformed errors and these were compared to the errors on the $c$ response determined from $Q$. Indeed the errors on $c$ used by the $D^{+}$and $H^{+}$inversions were greater than those formerly assigned. For the real part of $c$ the average enlargement was almost twice while on the imaginary part the errors were increased by a mean factor of 4 . Thus the $\chi^{2}$ misfit measure has been significantly affected by the transformations, by increasing the errors the $\chi^{2}$ 
is scaled down (Equation 7.2). This is not a desirable change to have taken place especially considering the approach of the study and methods used to make careful error estimates.

The calculation of apparent resisitivity from $Q$ is non-linear and therefore it is expected that some distortion will be introduced. On comparing the jackknife error bounds on the real and imaginary parts of $Q$ with the transformed error limits on the real and imaginary parts of apparent resistivity it was evident that for some frequency bands considerable bias had resulted and the degree of bias varied between frequency bands. The cause of most concern regarding the propagation of errors is the variation in bias for the different frequency bands. The programs used to invert the response (Section 7.2.2) use the $\chi^{2}$ misfit parameter to determine the 'best' model conductivity and therefore the model has some dependence on the errors. If the errors are affected by some common factor throughout the frequency bands then the misfit parameter will be altered but the model determined will be the same as for the original errors. However, inversion of the same response dataset with one set of errors and then with another unrelated set produces different models. Thus to some extent the models themselves will have been affected by the complicated nature of the error propagation. To avoid problems in the transformation of errors it would have been better to convert the individual response estimates to the form used by the inversion programs, apparent resistivity or $c$, and then use the jackknife method to calculate errors. The difficulty of switching between the forms of complex representation, real and imaginary parts to amplitude and phase, would mean the $c$ response rather than amplitude and phase of apparent resistivity should be used.

The result that will have been most adversely altered is the depth of penetration derived from $D^{+}$. The depth given is smaller than it should be if the plot of Figure 7.8 were shifted positively along the $\chi^{2}$ axis and so $840 \mathrm{~km}$ is a conservative estimate. The $D^{+}$method was initially used to confirm that the data fitted a 1-D conductivity distribution. Therefore if smaller error estimates were used the margin by which the misfit of the best fitting delta function model is lower than that expected must be reduced. However, as the 1-D models considered do fit the observed data well there is no doubt that a 1-D model is acceptable, i.e. the $\chi^{2}$ of the best delta function model is still smaller than the expected misfit.

The ultimate aim of this study was to produce a model for the average conductivity distribution of the mid-mantle. In Section 7.2.2 and 7.2.3 various models were derived which agreed with the observed response to varying degrees. The problem of converting errors should not be forgotten, the given $\chi^{2}$ misfit must be treated with caution and the models themselves will have been slightly affected but probably not by a significant 
amount. The $\mathrm{H}^{+}$inversion indicated that a reasonable fit to the data could be found with a simple model and that two layers may be sufficient. From the occam inversion models with a smooth or discontinuous rise in conductivity between 600 and $700 \mathrm{~km}$ were found to yield the best fit to the data which gave the basis for the forward modelling. Although the forward model $J$ of Figure 7.20 appears to fit the observed response well the two shortest period phase estimates with which it agrees are not consistent with the others and in light of the degradation of the $P_{1}^{0}$ power with frequency must be viewed more critically than the rest. The occam inversions which did not allow a discontinuity started with no a priori structure and derived smooth models very similar to the best two-layer models. From the models produced model I (Figure 7.19) was thought to be a fair final model to take for the global conductivity throughout the mid-mantle. Thus assuming the derived response to be valid a region of rapid increase in conductivity between 600 and $700 \mathrm{~km}$ depth is the major implied feature.

Recent work has been done on investigating lateral conductivity variations within the Earth and evidence has been found indicating some geographical variation (Petersons and Anderssen (1990), Schultz and Larsen (1990) and Schultz (1990)). Petersons and Anderssen reported an 'increase in the depth of the conducting mantle with decrease in geomagnetic latitude' for the European region of their study. However, they assume a $P_{1}^{0}$ source for observatories at higher geomagnetic latitudes than allowed in this study which are very likely to be corrupted by auroral effects as shown in Section 6.4.4 and discussed in Section 6.3. Therefore as the change in conductivity they find varies with distance from the auroral zone it is possible that the source field is partly responsible for the variation. The work of Schultz and Larsen (1990) and Schultz (1990) indicates the existence of variation in conductivity between sites for the upper and mid-mantle and in particular enhanced upper mantle conductivity for the Pacific region. The observatories used in their analysis and proposed lateral change in mantle conductivity are less likely to be due to source effects and would imply geographical structure in the upper and mid-mantle. Schultz (1990) concludes that there is a global transition from a 'relatively resistive upper mantle to conductive lower mantle' and that 'the data are consistent with a two or more order of magnitude jump in conductivity centered at a depth roughly coincident with the $670 \mathrm{~km}$ seismic discontinuity'. This is very similar to the result of the current study, i.e. a step in conductivity between 600 and $700 \mathrm{~km}$ and contrast of approximately two orders of magnitude. Therefore from the lateral variations found by Schultz and Larsen (1990) and Schultz (1990), the average finding agrees well with this present work which neglects lateral structure. Thus it would appear that it is possible to assume a radially symmetric mid-mantle to estimate the global conductivity depth profile. 
The work presented in this thesis starts with the premise that the Earth may be considered one-dimensional for the frequency range of the derived response. The surface of the Earth varies in nature with geographical location and geophysical studies indicate regional variations to at least $400 \mathrm{~km}$ depth. A certain degree of lateral variation should not be discounted below this depth and it may be unwise to state too boldly the significance of a particular depth in defining a limit of laterally variable conductivity. However, non-radial variations in the makeup of the Earth undoubtedly decrease as depth is increased and the objective of this study was to determine if it is possible to obtain a 1-D response function from magnetic signals varying in time from roughly a month to a day period penetrating the mid-mantle. It has been shown (Section 7.2.2) that it is possible to produce a range of one-dimensional models that fit the data very well. The only word of caution that should be mentioned is the reliability of the error estimates as it is discussed above how they have been effected by the switch between different response types. Although the errors have been affected the ability of the response function to be modelled in one-dimension is not thought to be discredited. If so lateral variations have not influenced the response to such an extent that a 1-D Earth model has to be rejected. 
Figure 7.11. Result of Occam Inversion: Model A, Continuous Conductivity Model, First Derivative minimised, Tolerated Misfit $=1.0$.
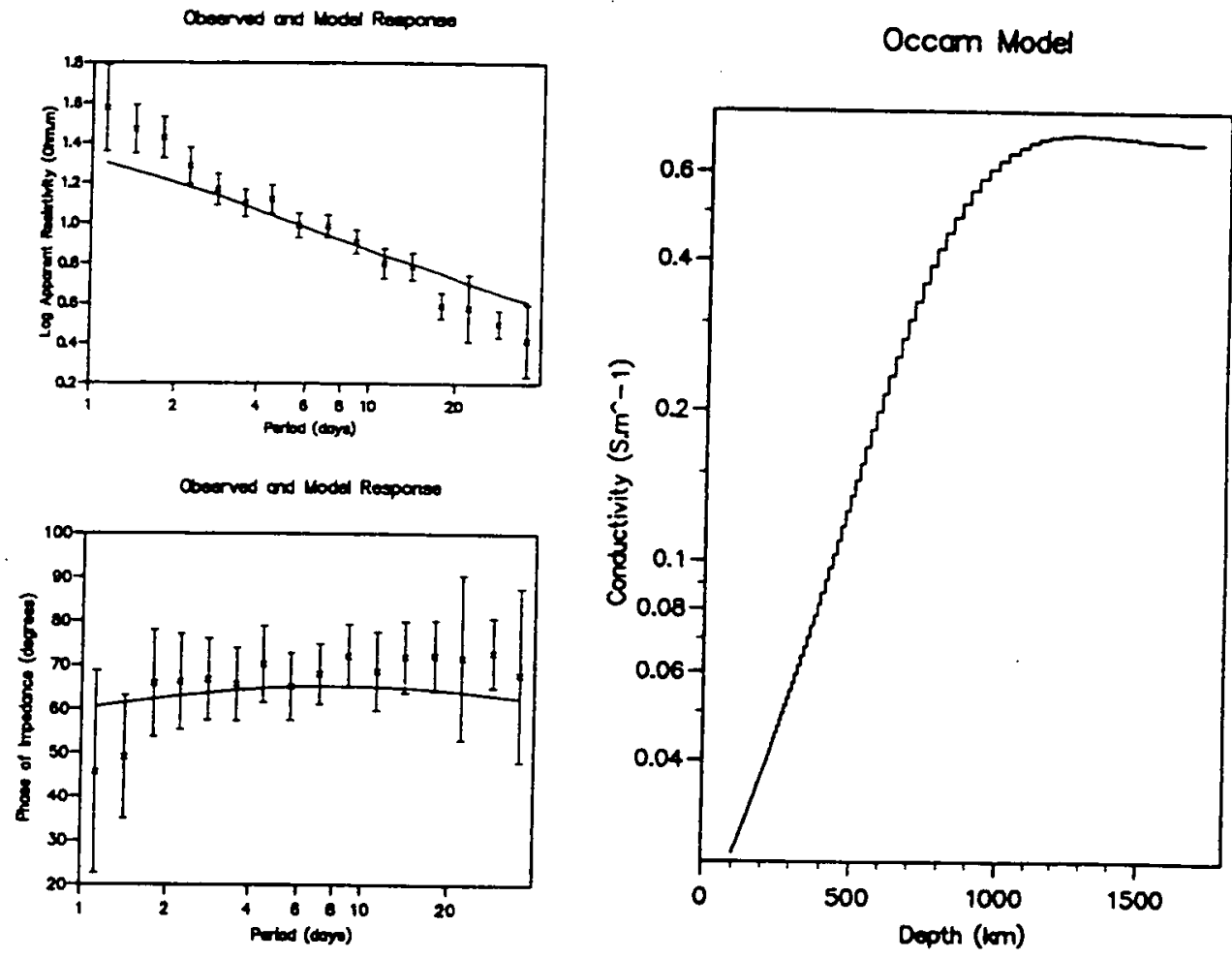
Figure 7.12. Result of Occam Inversion: Model B, Continuous Conductivity Model, First Derivative minimised, Tolerated Misfit $=0.8$.
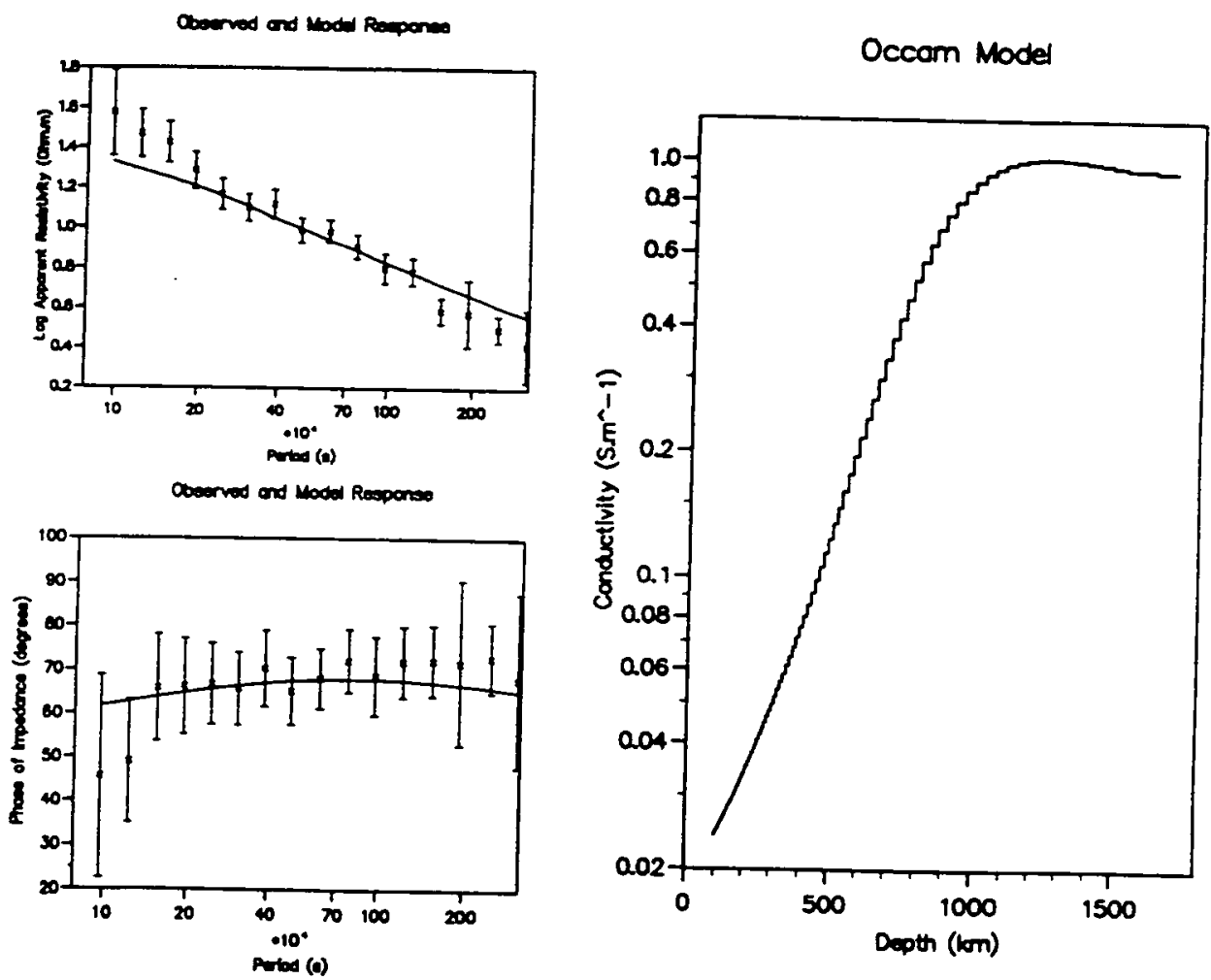
Figure 7.13. Result of Occam Inversion: Model C, Continuous Conductivity Model, First Derivative minimised, Tolerated Misfit $=0.6$.
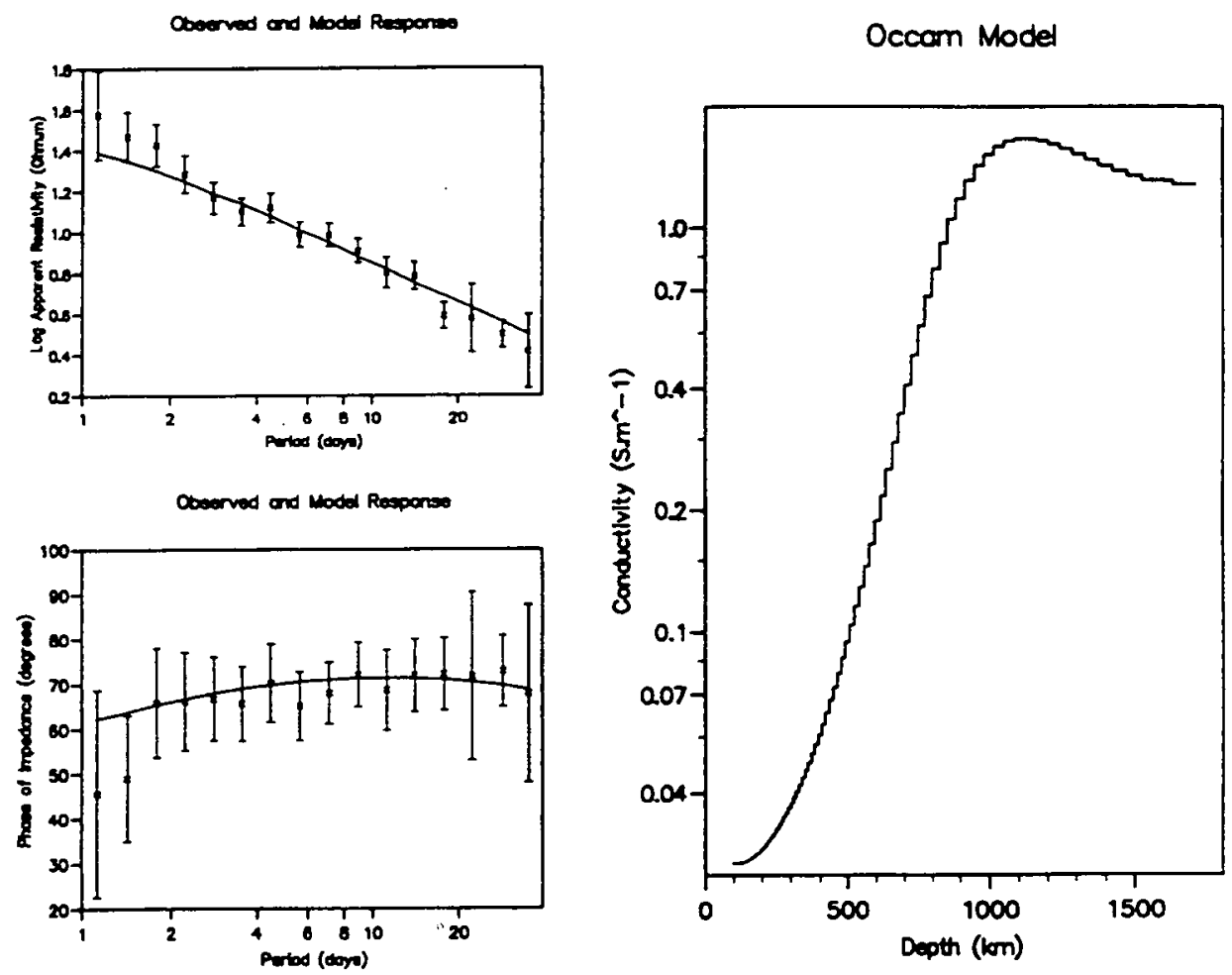
Figure 7.14. Result of Occam Inversion: Model D, Continuous Conductivity Model, Second Derivative minimised, Tolerated misfit $=1.0$.
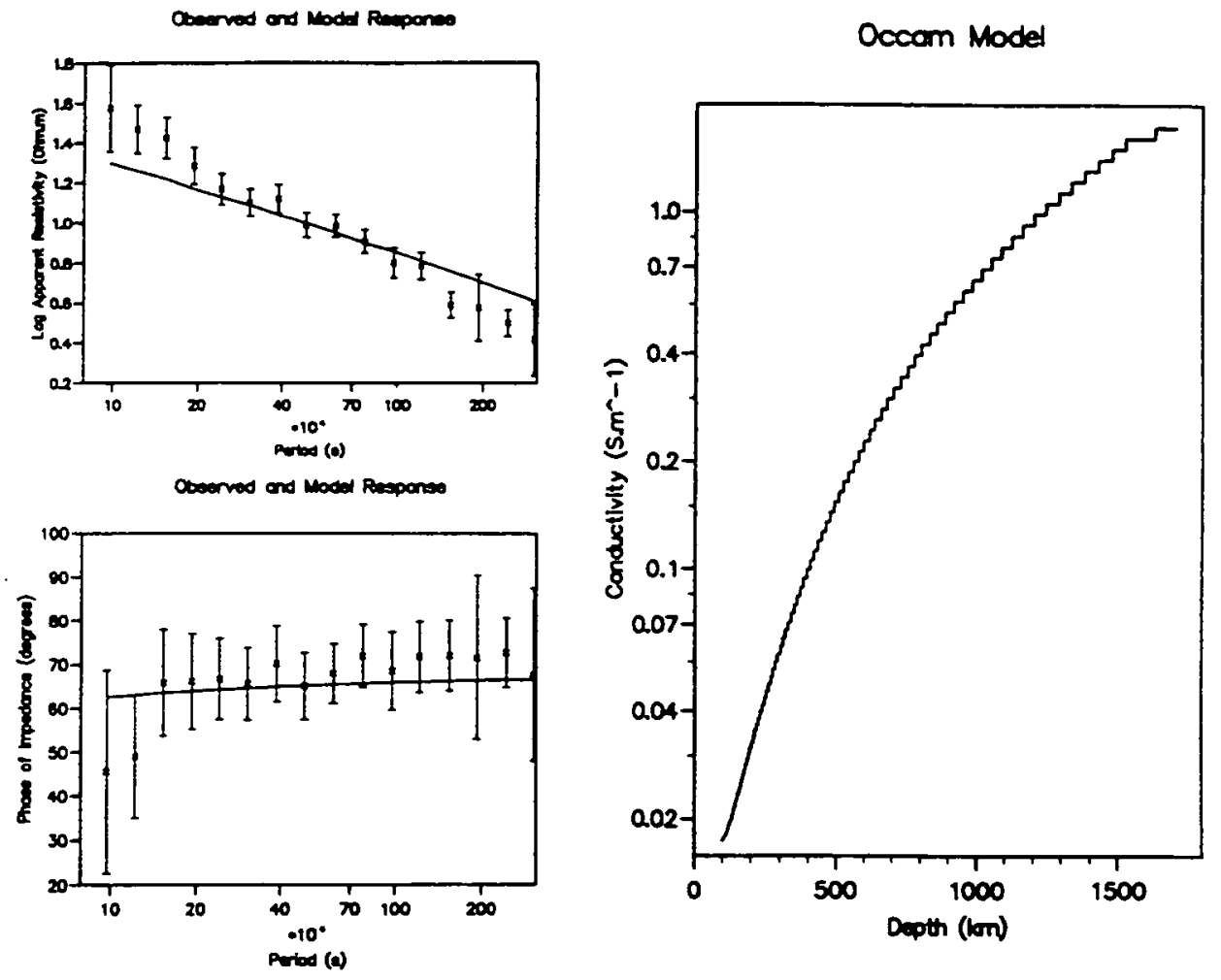
Figure 7.15. Model E: Result of Occam Inversion allowing a Discontinuity in Conductivity at $587 \mathrm{~km}$. First Derivative minimised, Tolerated Misfit $=1$.
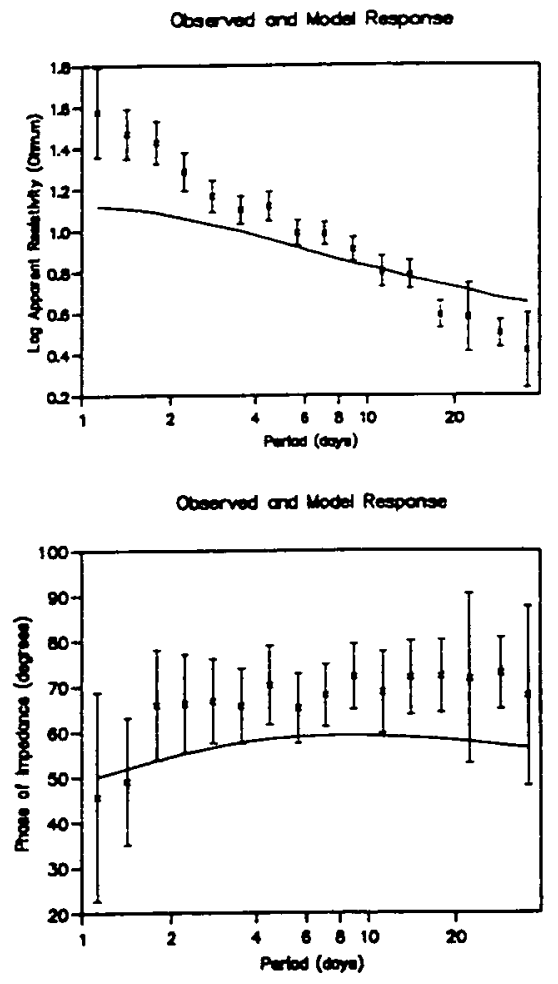

Occam Model

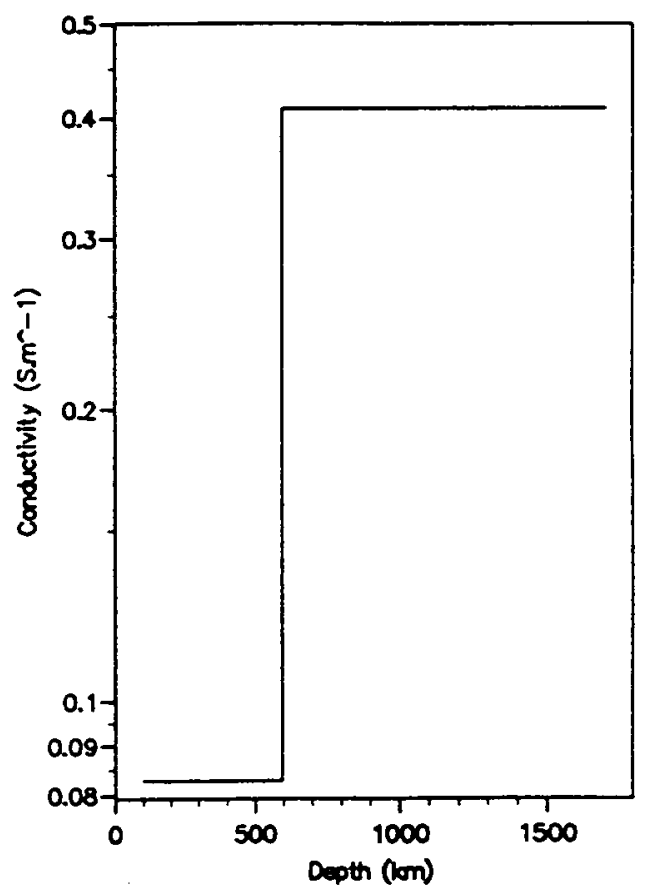


Figure 7.16. Model F: Result of Occam Inversion allowing a Discontinuity in Conductivity at $626 \mathrm{~km}$. First Derivative minimised, Tolerated Misfit $=1$.
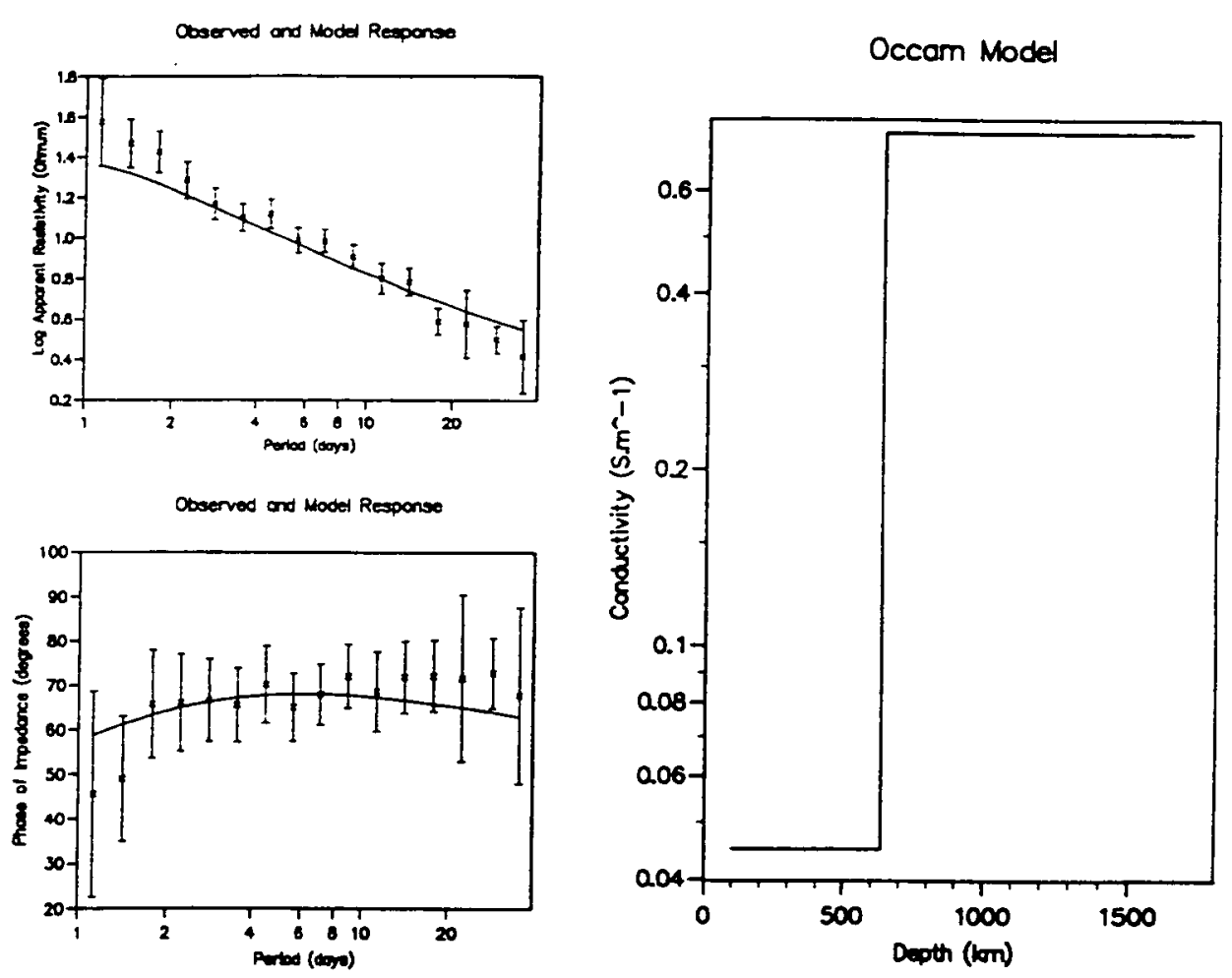
Figure 7.17. Model G: Result of Occam Inversion allowing a Discontinuity in Conductivity at $668 \mathrm{~km}$. First Derivative minimised, Tolerated Misfit $=1$.
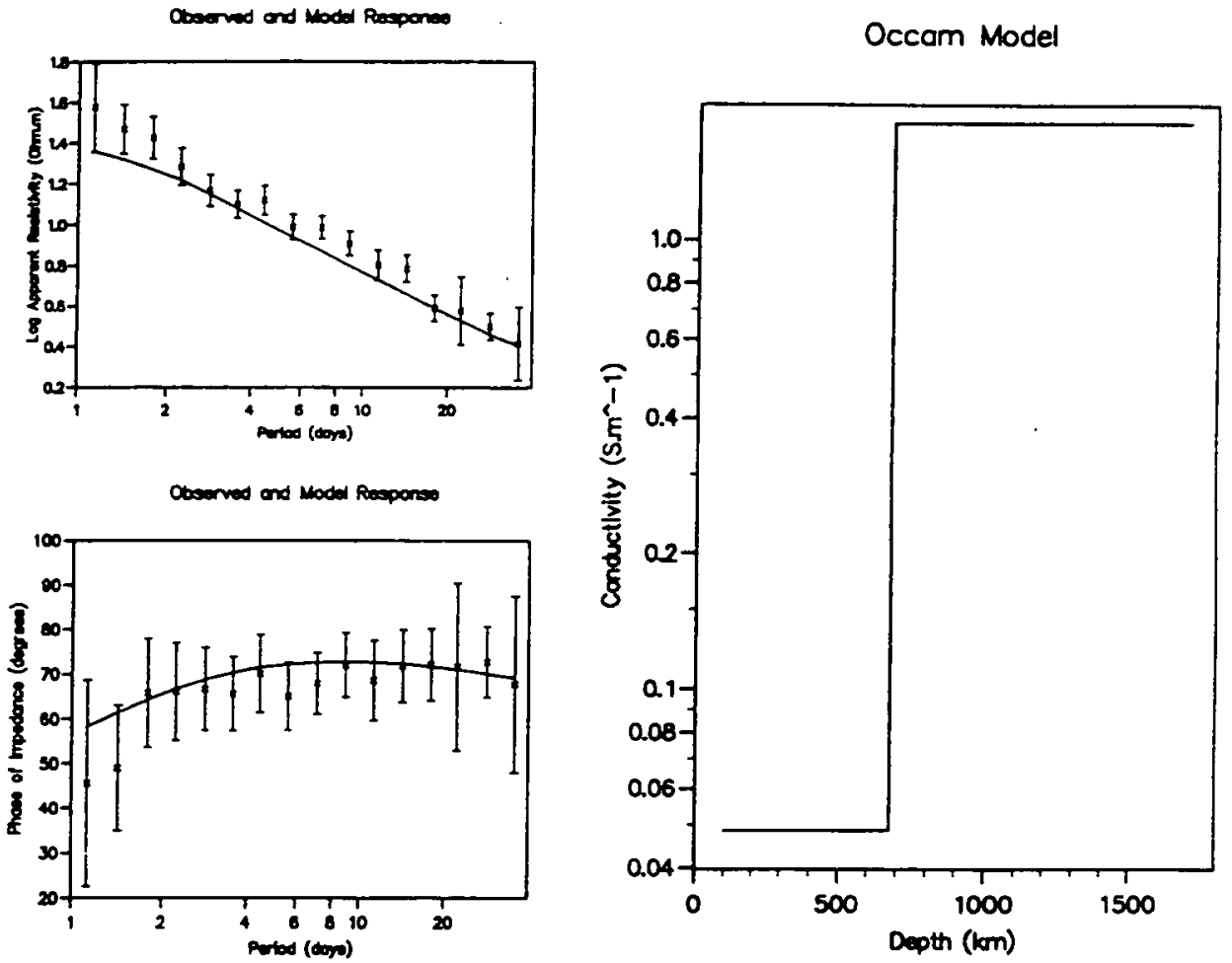
Figure 7.18. Model H: Result of Occam Inversion allowing for a Discontinuity in Conductivity, minimising the Second Derivative, Tolerated Misfit $=1$.
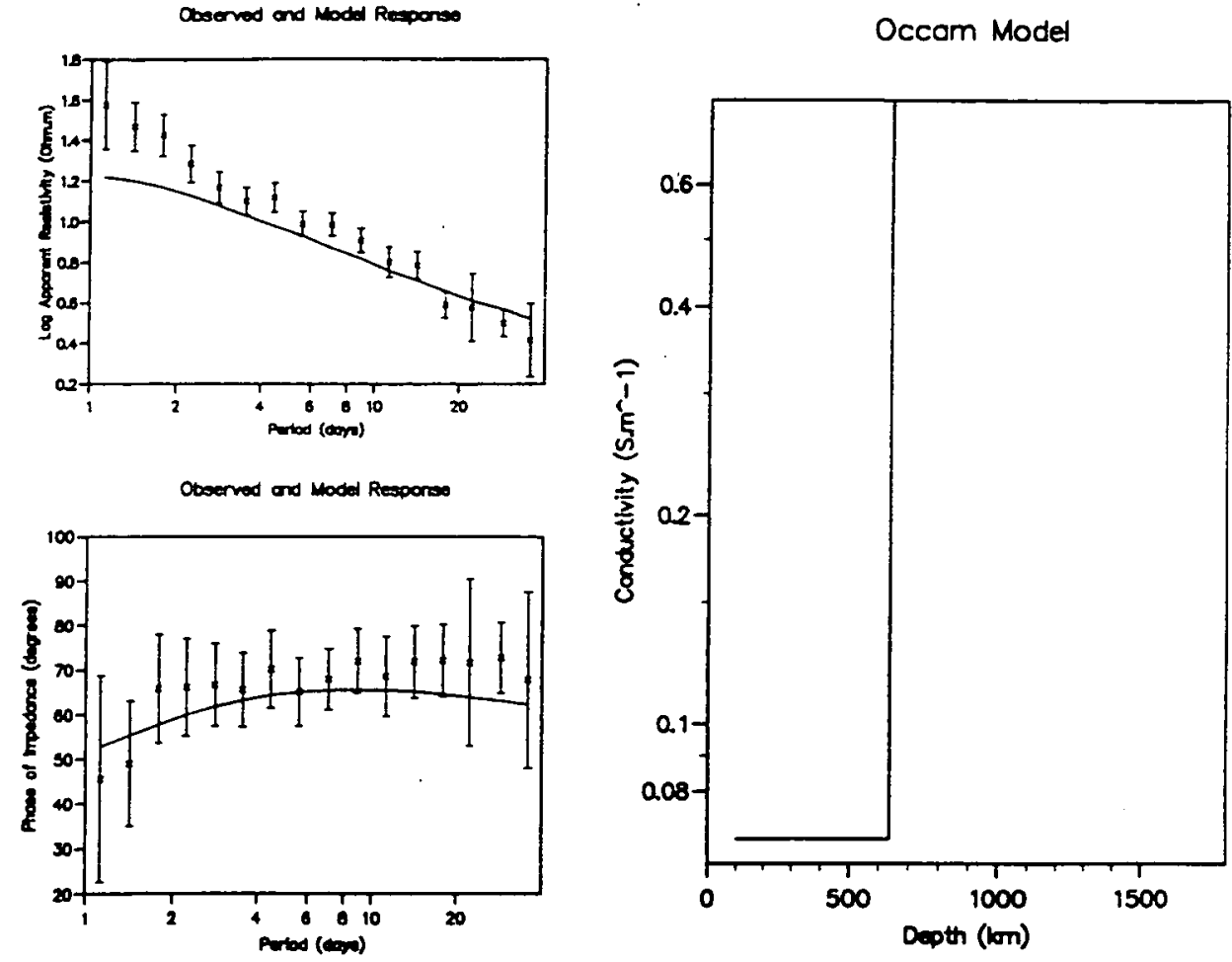
Figure 7.19. Model I: Produced by Forward Modelling. Upper layer Conductivity $0.03 \mathrm{Sm}^{-1}$, Lower Layer $1.43 \mathrm{Sm}^{-1}$, Discontnuity at $636 \mathrm{~km}$.
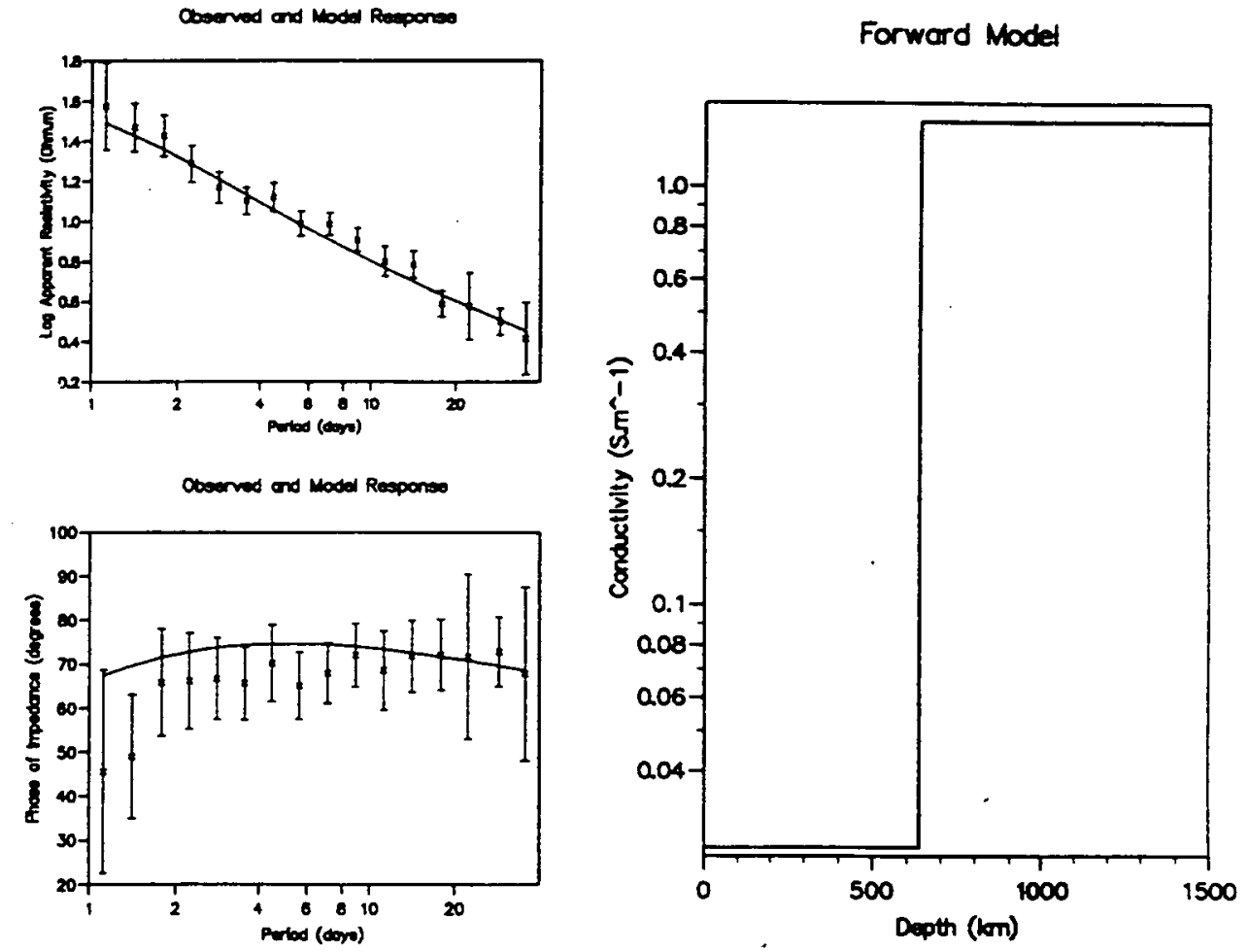
Figure 7.20. Model J: Produced by Forward Modelling. Upper layer Conductivity $0.04 \mathrm{Sm}^{-1}$, Lower Layer $3.33 \mathrm{Sm}^{-1}$, Discontnuity at $800 \mathrm{~km}$.
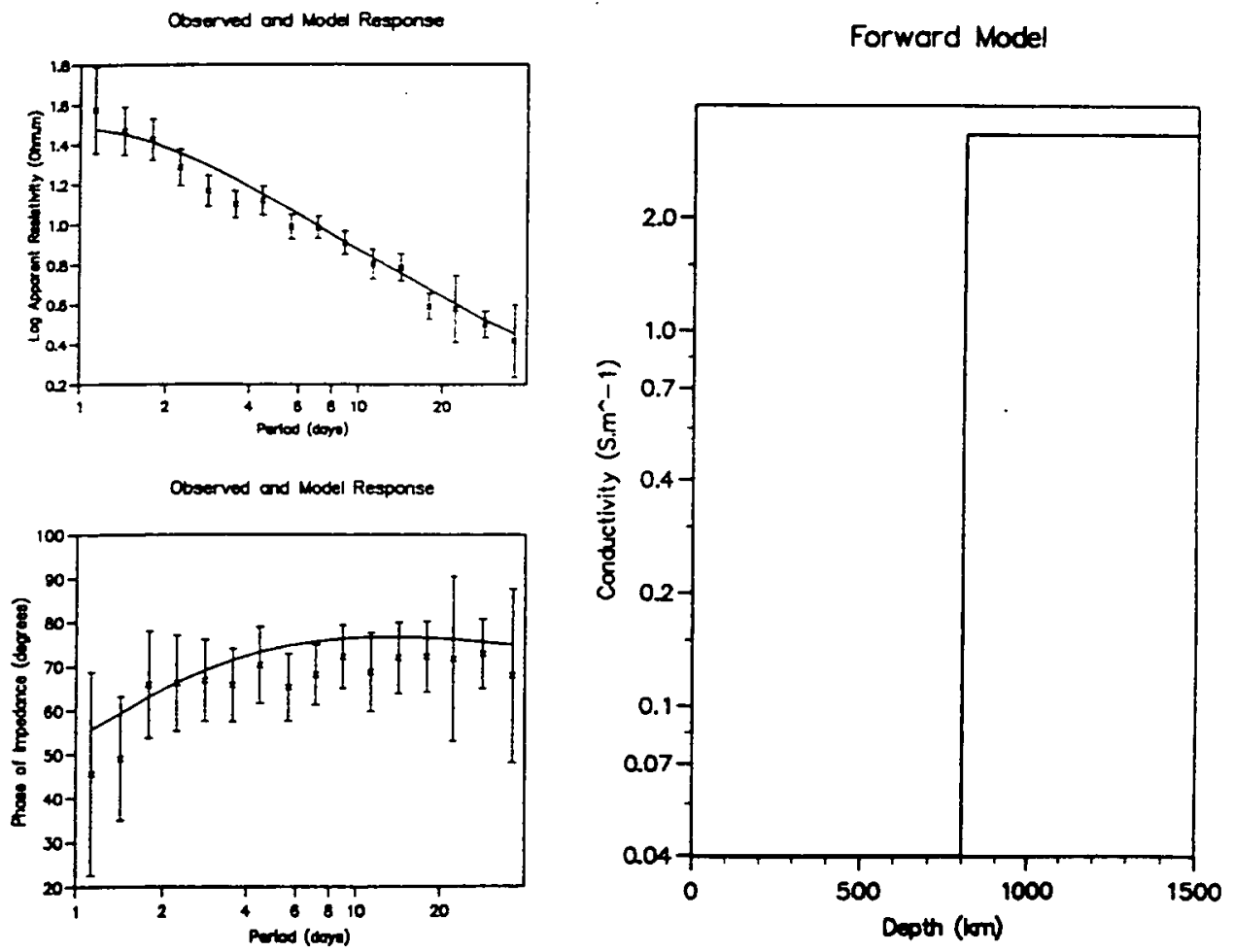
Figure 7.21. A Two-Layer Model and Response. Upper Layer Resistivity $=33.2 \Omega \mathrm{m}$, Lower Layer $=0.7 \Omega \mathrm{m}$, Discontinuity $=680 \mathrm{~km}$.

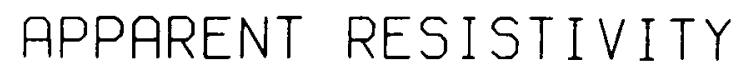

MODEL

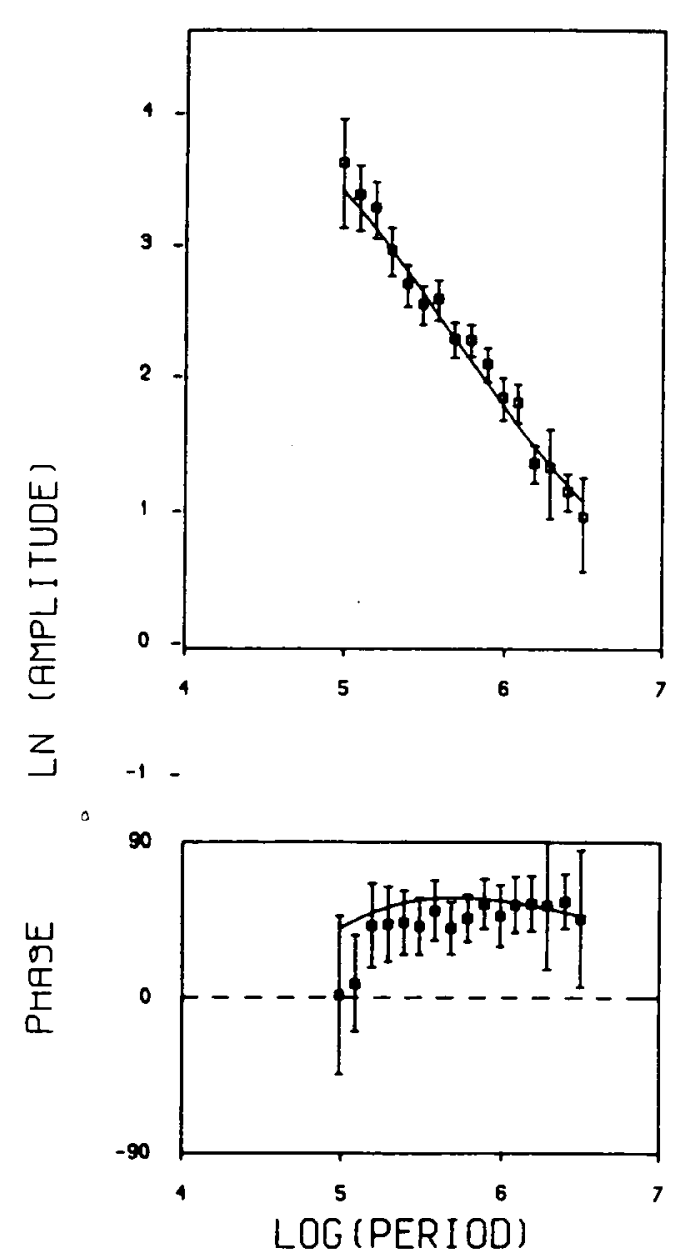

LOG (RESIST IVITY)

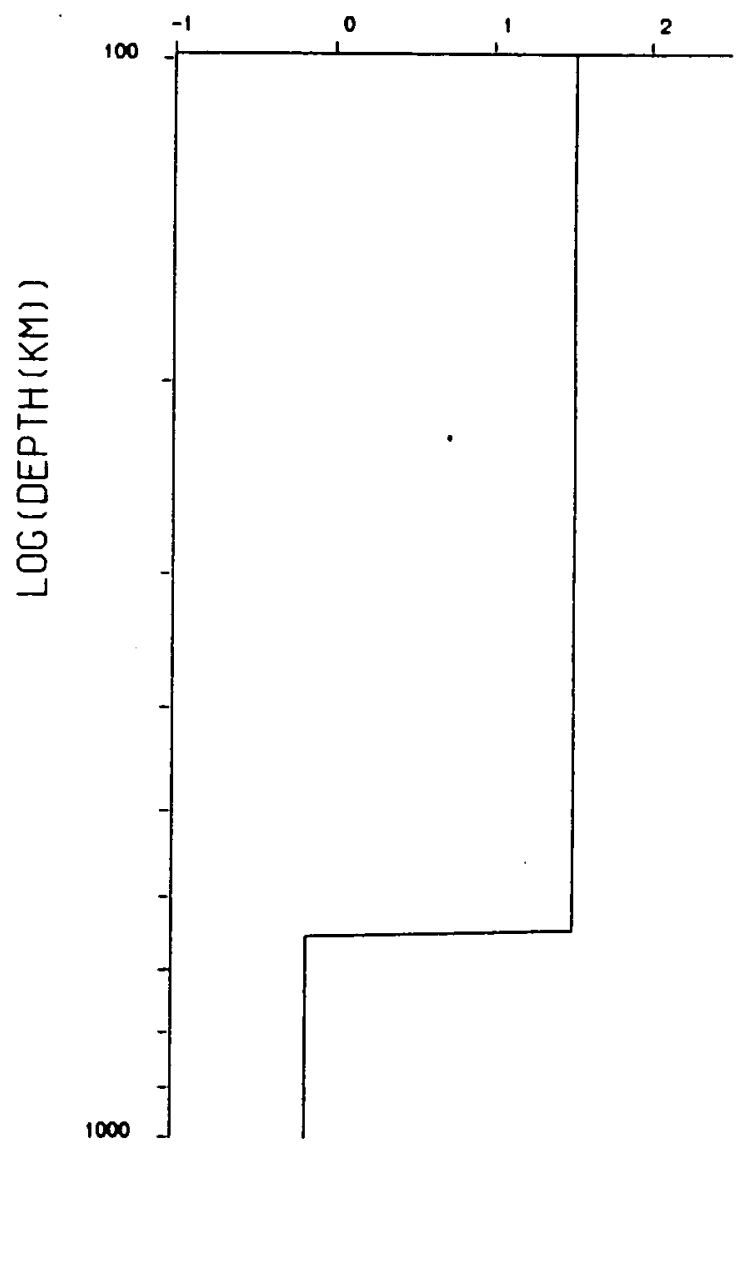


Figure 7.22. Trade-Off Diagram (a) and Model Average, Relative Error 0.3 (b) for Two-Layer Model.

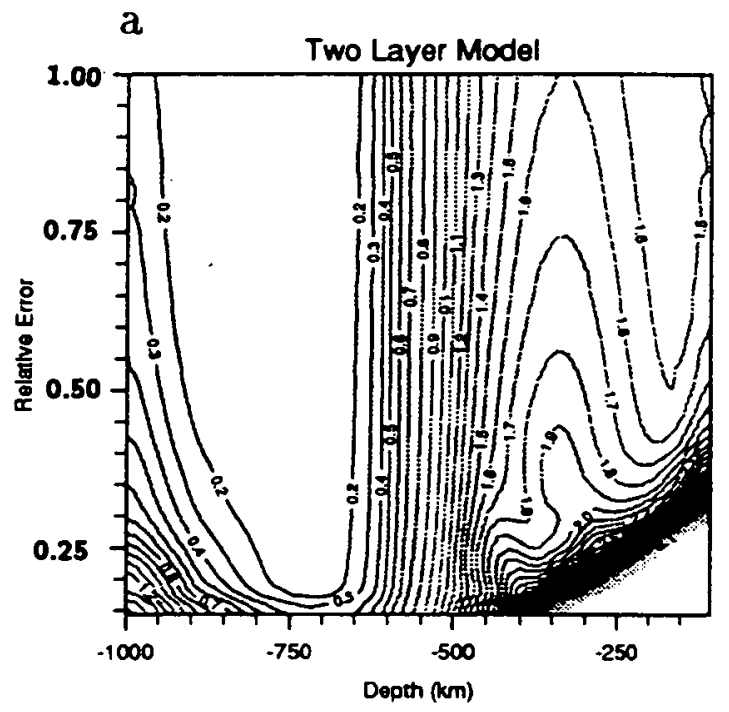

b MODEL AVERAGE

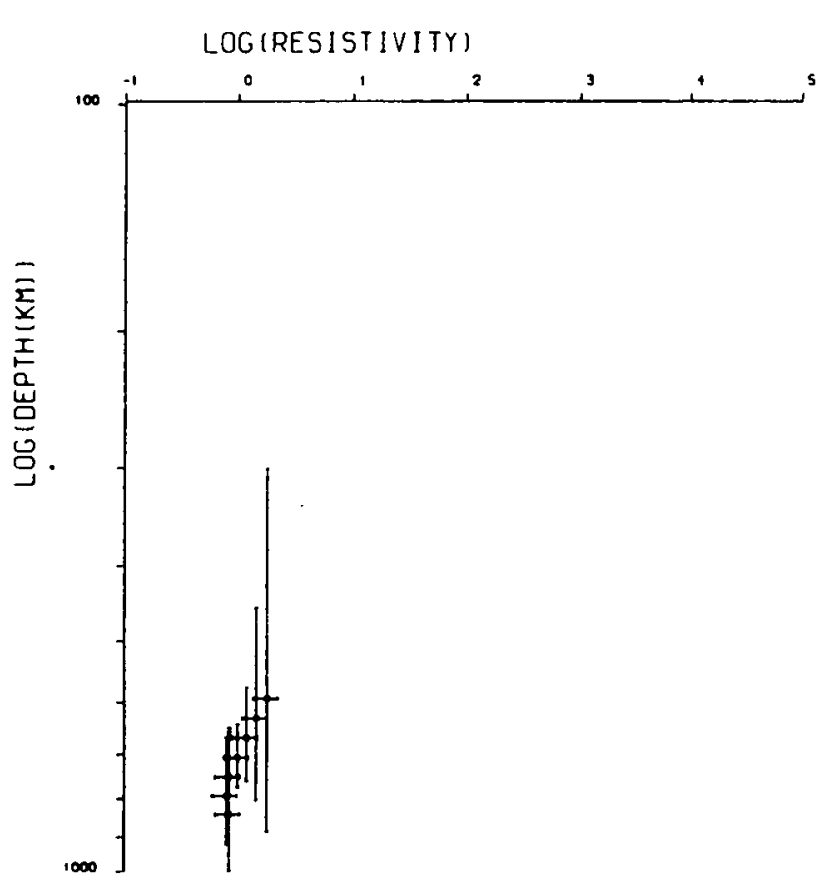


Figure 7.23. A 'Smooth' Model and Response.

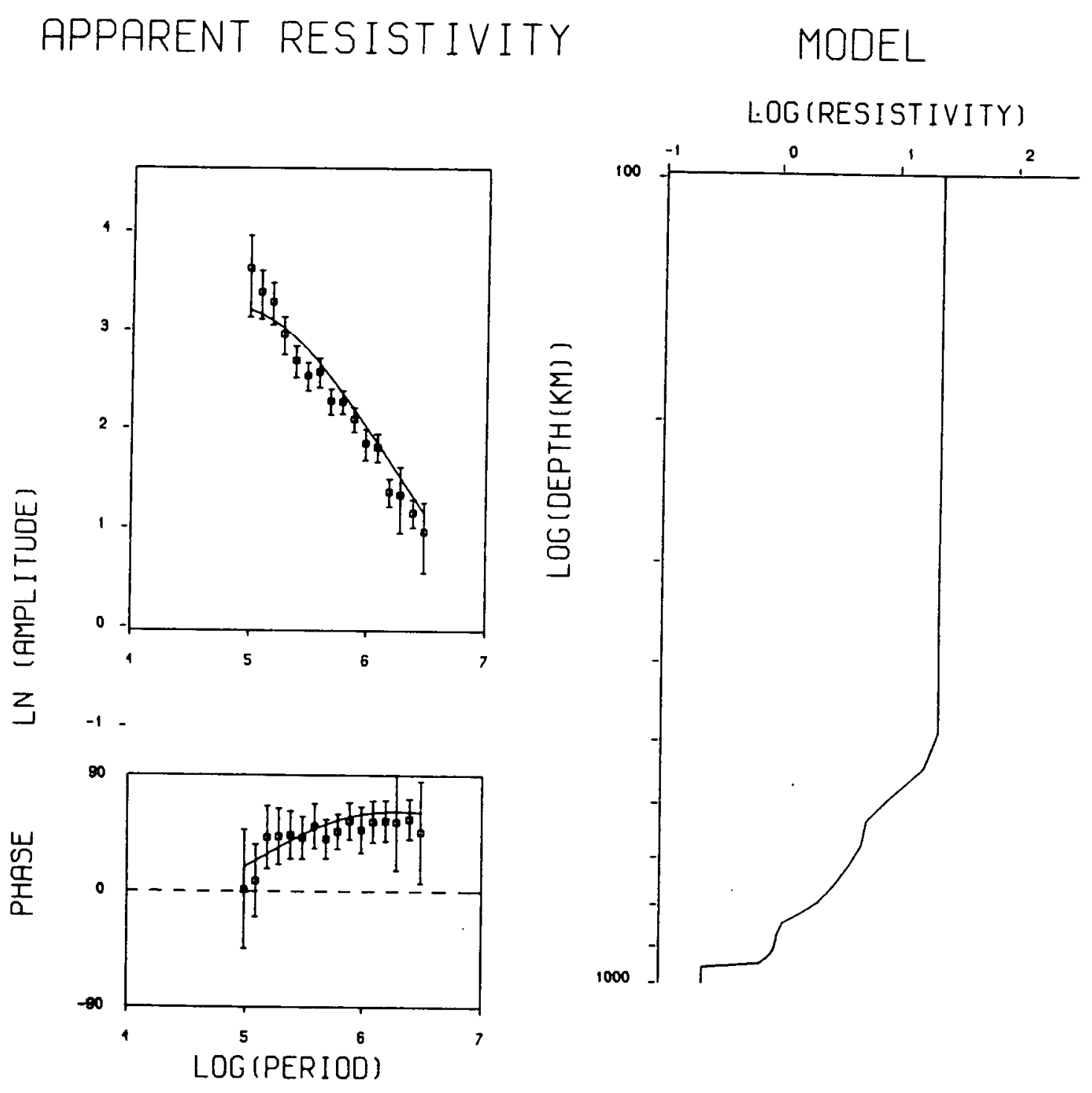


Figure 7.24. Trade-Off Diagram (a) and Model Average, Relative Error 0.3 (b) for Smooth Model.

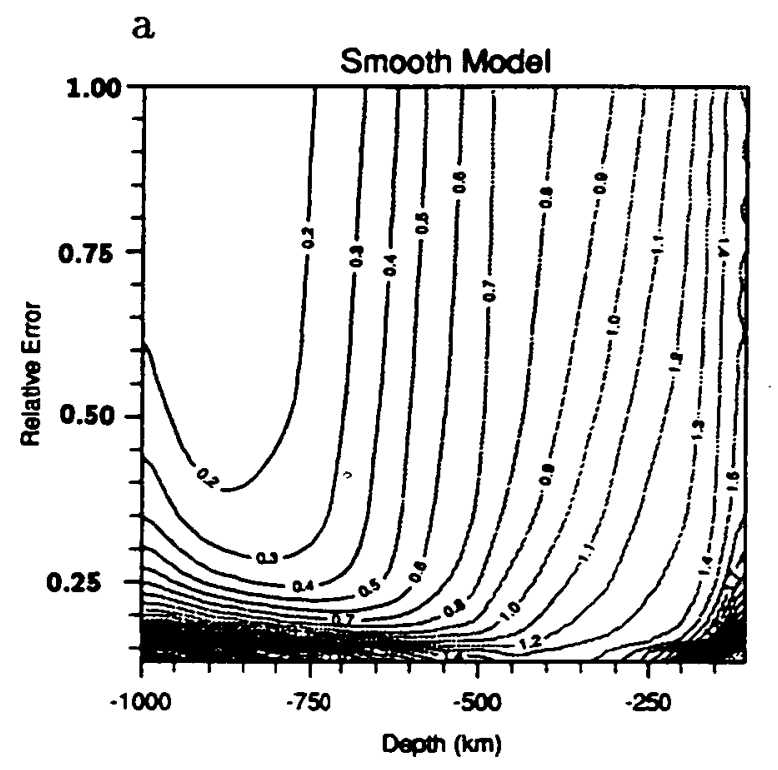

b MODEL AVERACEE

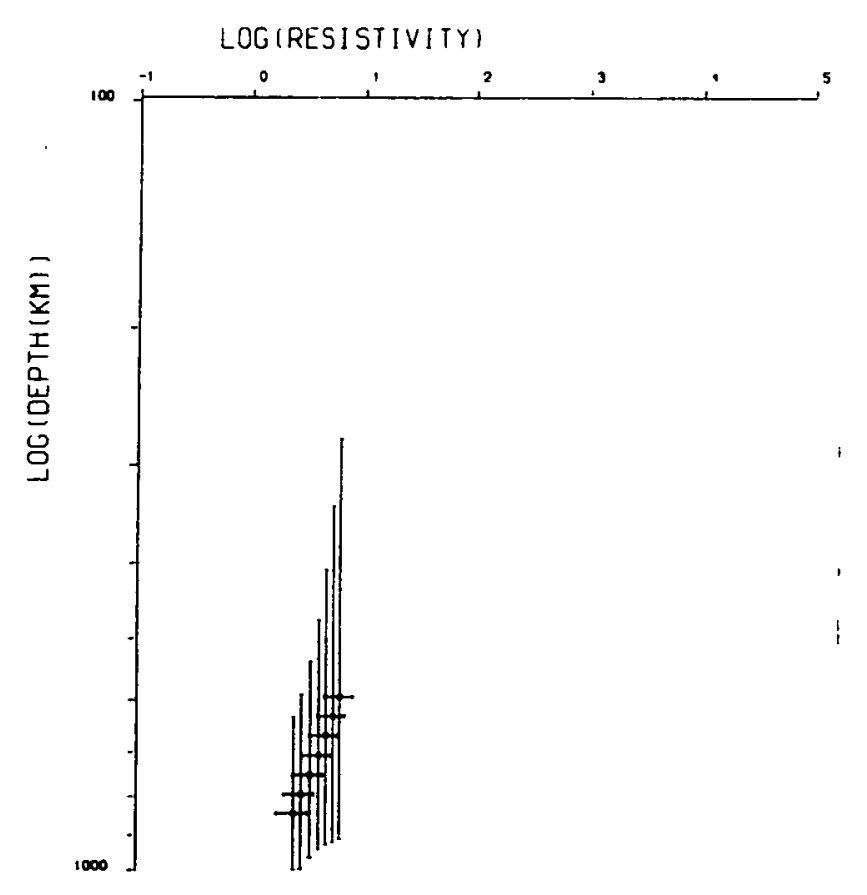




\section{Chapter 8}

\section{Conclusions}

The main objective of this study was to determine a well defined response function resulting from the propagation of geomagnetic variations into the Earth's mantle. From the analysis of 44 magnetic storms this has been achieved with the uncertainty in the response found from the distribution of estimates. The use of numerous storms has then been shown to be a worthwhile technique. The main findings of the work are given below.

Firstly before the analysis of a large body of data may take place steps must be made to ensure the data is as error free as possible. Due to the disturbed nature of magnetic storm records no automatic scheme was found and assessment had to done visually. Marshall (1980) also noted the importance of examination by eye. Once the type and occurrence times of errors had been established corrections were carried out (Chapter 4) and an effort made to interpolate for missing data.

The Fourier analysis of the observatory records (Chapter 5) before SHA seems the most suitable way to proceed to reduce the database size. The behaviour of the Fourier coefficients was investigated using a synthetic storm function. The effect of the timeshift theorem and 'beating' in the frequency domain when the record included two sudden commencements was observed. However, it was concluded that these phenomena would not harm the subsequent SHA.

As in past work the $P_{1}^{0}$ term proved to be dominant for the $X$ component and was also thought to be significant in $Z$ after examination of the spatial form of the fc's (Chapter 6). A $P_{1}^{0}$ spherical harmonic structure was therefore taken to describe the source field though its prominence was reduced at the high end of the considered frequency range. The robust IRLS method was useful in determining the most appropriate $P_{1}^{0}$ fit to the Fourier coefficients. This method reduced the undesirable effects of the more corrupt high latitude and locally anomalous observatory Fourier coefficient 
estimates. Degradation of the $P_{1}^{0}$ spatial form near to the $35^{\circ}$ and $145^{\circ}$ co-latitude limits suggests this range should be further restricted even though fewer observatories would then be available.

Robust techiques were also beneficial in the estimation of the average global response function (Section 7.1) though there was not a great difference between the estimate of response given by the ordinary mean and that of the robust average within the frequency bands. This was because the response values found from the storms were near normally distributed. However, to achieve the best possible estimate of response the robust average was chosen as then any departures from the normal have been downweighted. The robust jackknife errors were also adopted being more resistant to the presence of outliers while assigning conservative bounds when the distribution within the frequency band was almost normal. The derived average response was physically acceptable using the entire set of individual storm estimates. This was a pleasing result obtained without rejecting data which did not comply with the physics.

Finally the data was used in an attempt to deduce the conductivity distribution of the mid-mantle (Section 7.2). The $D^{+}$inversion method indicated that the data did conform to a 1-D conductivity distribution and that the maximum depth of penetration was $840 \mathrm{~km}$. However, as discussed the quoted $\chi^{2}$ misfit of the models should be larger due to the enlargement of the errors in transforming between different forms of response. This means the actual penetration depth is greater than that found. As the bias in error bounds was found to differ between frequency bands the determined models will not be exactly as if errors truly representative of those originally found on $Q$ had been used. The $\mathrm{H}^{+}$method revealed that models with a rapid rise in conductivity fitted the data which was confirmed by the occam inversion. The occam inversion produced a good fit to the response when the smoothness contraint was obeyed throughout the depth range and when a discontinuity was allowed. These models suggest a rapid rise in conductivity most probably between 600 and $700 \mathrm{~km}$. The forward model I of Figure 7.19 was suggested as a reasonable final model which estimates the upper section to be just above $10^{-2} \mathrm{Sm}^{-1}$ with the region below the transition around 1 $\mathrm{Sm}^{-1}$. This model superimposed on the envelope of previous conductivity models, as shown in Figure 1.3, is presented in Figure 8.1. It should, however, be noted that the true conductivity is very unlikely to be such a two-layer distribution but that the main features are thought to be close to this model. Of the previous models for the midmantle the final model of this study is closest to the discontinuous model of Lahiri and Price (1939), their model $e$, which gave a jump in conductivity in the vicinity of 600 $\mathrm{km}$ depth from approximately $10^{-3} \mathrm{Sm}^{-1}$ to $1 \mathrm{Sm}^{-1}$. Thus the contrast in conductivity is smaller in the model presented here. 
Figure 8.1. Model I superimposed on the 'The bounds on the Average Conductivity for the Mid-Mantle'.

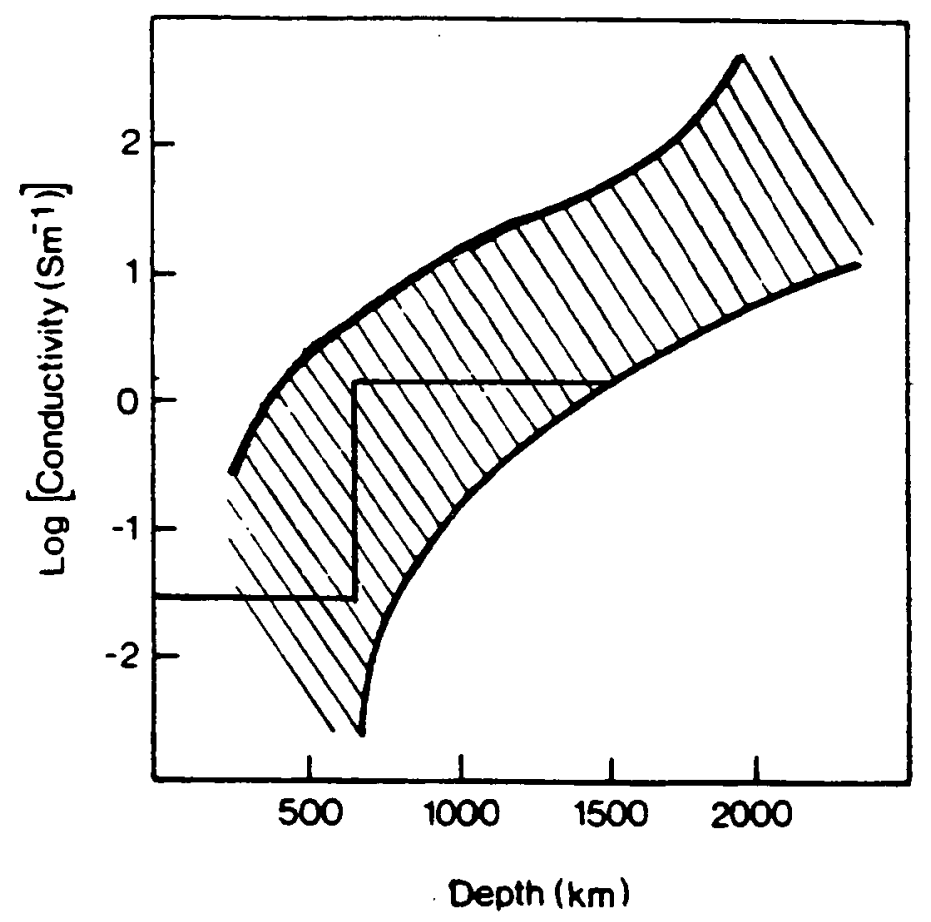




\section{Chapter 8}

Because of the detrimental effect on the errors of changing between different forms of response such conversions should be kept to the minimum and thus the inversions should be carried out using the $c$ response. As stated in Section 7.2.5 the models will have been influenced but not by a significant amount. It would have been preferable to convert the individual storm estimates to $c$ and then determine the average response and associated errors and so avoid the conversion from $Q$.

It therefore appears that a global response function for the mid-mantle is valid and that any geographical variations in conductivity do not seem large enough to have adversely affected this global study. The value of determining the Earth's response as accurately as possible was highlighted by Parker (1970) and echoed by Banks (1972). It is believed that the average response and original error estimates presented here are a step towards this goal. Additionally a model for the variation of conductivity in the mid-mantle has been proposed which by chance or physical significance agrees very well with the seismic discontinuity at $650 \mathrm{~km}$. 


\section{References}

Achache, J., J.L. Le Mouel and V. Courtillot (1981) Long-period geomagnetic variations and mantle conductivity: an inversion using Bailey's method. Geophys. J. R. astr. Soc. 65 579-601.

Ainsworth (1987) QNTNOR subroutine written for the N.E.R.C. funded project 'The Electrical Conductivity of the Upper Mantle.' R.J. Banks and J.A. Ainsworth, Lancaster University.

Akasofu, S.I. and S. Chapman (1972) Solar-Terrestrial Physics. Oxford University Press.

Anderson, D.L. (1989) Theory of the Earth. Blackwell Scientific Publication.

Anderssen, R.S. and E. Seneta (1969) New Analysis for the Geomagnetic Dst Field of the Magnetic Storm on June 18-19, 1936. J. Geophys. Res. 74 2768-2773.

Anderssen, R.S., H.A. Doyle, H.F. Petersons and E. Seneta (1970) On the Smoothing and Spherical Harmonic Analysis of the Storm of September 25, 1958. J. Geophys. Res. 75 2569-2577.

Backus, G. and F. Gilbert (1968) The Resolving Power of Gross Earth Data. Geophys. J. R. astr. Soc., 16, 169-205.

Bailey, R.C. (1970) Inversion of the Geomagnetic Induction Problem. Proc. $R$. Soc. London, (A), 315, 185-194.

Banks, R.J. (1969) Geomagnetic Variations and the Electrical Conductivity of the Upper Mantle. Geophys. J. R. astr. Soc. 17 457-487. 
Banks, R.J. (1972) The Overall Conductivity Distribution of the Earth. J. Geomag. Geoelectr. 24 337-351.

Bassom, A.P. and R.J. Jady (1988) An inversion method of Gel'fand Levitan type for the electromagnetic induction problem. Geophysical Journal International, 82, 111-123.

Bath, M. (1974) Developments in Solid Earth Geophysics 7, Spectral Analysis in Geophysics. Elsevier Scientific Publishing Company, Amsterdam.

Benkova, N.P. (1953) Magnetic storms and systems of electrical currents. (in Russian) Pros. NIISM 10 (20) Leningrad. Gidrometizdat 156.

Berdichevski, M.N., B. Fainberg, N.M. Rotanova, J.B. Smirnov and L.L. Vanyan (1976) Deep Electromagnetic Investigations. Ann. Geophys. 32, 143-155.

Boteler, D.H. (1991) Predicting Geomagnetic Disturbances on Power Systems. EOS April 2nd.

Brett, L. (1988) Methods of Spherical Harmonic Analysis. Q. J. R. Astr. Soc. 29, 129-155.

Chapman, S. and J. Bartels (1940) Geomagnetism. Clarendon Press, Oxford.

Chapman, S. and A.T. Price (1930) The electric and magnetic state of the interior of the earth, as infered from terrestrial magnetic variations. Phil. Trans. Roy. Soc. London (A), 228, 233-45.

Chapman, S. and T.T. Whitehead (1922) The influence of electrically conducting material within the earth on various phenomena of terrestrial magnetism. Trans. Phil. Soc. Cambridge 22 463-482.

Chave, A.D., D.J. Thomson and M.E. Ander (1887) On the Robust Estimation of Power Spectra, Coherences, and Transfer Functions. J. Geophys. Res. 92 B1 633-648. 
Chave, A.D. and D.J. Thomson (1988) Some Comments on Magnetotelluric Response Function Estimation. J. Geophys. Res. 84 14215-14225.

Constable, S.C., R.L. Parker and C.G. Constable (1987) Occam's Inversion: A practical algorithm for generating smooth models from electromagnetic sounding data. Geophyics, 52 3, 289-300.

Decker, R. and B. Decker (1982) Volcanoes and the Earth's Interior. Scientific American, W.H. Freeman and Company, San Francisco.

Devane, J.F. (1977) Global electrical conductivity from a magnetic storm. Acta Geodaet., Geophys. et Montanist. Acad. Sci. Hung. Tomus 12 (1-3) 369-375.

Duffin, W.J. (1990) Electricity and Magnetism. McGraw Hill Book Company (UK) Ltd.

Draper N.R. and H. Smith (1981) Applied Regression Analysis. John Wiley and Sons.

Efron, B. and G. Gong (1883) A Leisurely Look at the Bootstrap, the Jackknife, and Cross-Validation. The American Statistician, 37 1, 36- .

Egbert, G.D. and J.R. Booker (1986) Robust estimation of geomagnetic transfer functions. Geophys. J. R. astr. Soc. 87 173-194.

Fougere, P.F. (1969) Spherical Harmonic Analysis 3. The Earth's Magnetic Field, 1900-1965. J. Geomag. Geoelectr. 21 685- .

Grafe, A. (1963) Die Bedeutung der Abweichungen geomagnetischer Tagesmittel vom sogenannten Normalwert fur die Analyse des geomagnetischen Ringstromeffektes. Dtsch. Akad. Wiss. Berlin, Geomagn. Inst. Potsdam, Abh., 31 7-53.

Graham, G. (1724) An Account of Observations Made of the Variation of the Horizontal Needle at London in the Latter Part of the Year 1722 and Beginning 1723. Phil. Trans. Roy. Soc. London 383, 96-107.

Grant, I.S. and W.R. Phillips (1982) Electromagnetism. John Wiley and Sons. 
Gubbins, D. (1983) Geomagnetic field analysis - I. Stochastic inversion. Geophys. J. R. astr. Soc. 73, 641-652.

Harris, F.J. (1978) On the Use of Windows for Harmonic Analysis with the Discrete Fourier Transform. Proceedings of the IEEE 66 51-83.

Heinz, D.L. (1991) Split decision on the mantle. Nature 351, 346-347.

Hoaglin, D.C., F. Mosteller and J.W. Tukey (1983) Understanding Robust and Exploratory Data Analysis. John Wiley and Sons, New York.

Hobbs, B.A. (1982) Automatic Model for finding the one-dimensional magnetotelluric problem. Geophys. J. R. astr. Soc., 168, 253-266.

Hobbs, B.A. (1983) Inversion of Broad Frequency Band Geomagnetic Response Data. J. Geomag. Geoelectr., 35, 723-732.

Hobbs, B.A. (1987) Conductivity Profiles from Global Data. PAGEOPH 125 2/3, 393-407.

Hobbs, B.A. (1992) Terminology and Symbols for use in studies of electromagnetic induction in the earth. Surveys in Geophysics, in press.

Huber, P.J. (1964) Robust estimation of a location parameter. Ann. Math. Stat. 35, 73-101.

Jady, R.J. (1875) Electromagnetic Induction in the Mantle by Aperiodic Variations. Geophys. J. R. astr. Soc. 40, 67-83.

Jady, R.J., R.T. Marshall and K. Morgan (1979) Comparison of analyses of Dst variables. Phys. Earth Planet. Int. 20 6-10.

Kamide, Y. (1891) The Relationship between Storms and Substorms. Paper presented at 20th General Assembly of IUGG in Vienna.

Kanesewich E.R. (1981) Time Sequence Analysis in Geophysics. The University 
of Alberta Press.

Kleiner, B. and T.E. Graedel (1980) Exploratory data analysis in the geophysical sciences. Rev. Geophys. 18, 699-717.

Lahiri, B.N. and A.T. Price (1939) Electromagnetic induction in non-uniform conductors and determination of the conductivity of the Earth from terrestrial magnetic variations. Phil. Trans. Roy. Soc. London A237, 509-540.

Langel, R.A. (1987) The Main Field. In: Jacobs J.A. (ed.) Geomagnetism. Academic Press, London.

Langel, R.A., D.R. Barraclough, D.J. Kerridge, V.P. Golovkov, T.J. Sabaka and R.H. Estes (1988) Definitive IGRF Models for 1945, 1950, 1955 and 1960. J. Geomag. Geoelectr. 40, 645-702.

Larsen, J.C. (1877) Removal of local surface conductivity effects from low frequency mantle response curves. Acta Geodaet., Geophys, et Montanist. Acad. Sci. Hung. Tomus 12, 183-186.

Lewis, T. and N.I. Fisher (1982) Graphical methods for investigating the fit of a Fisher distribution to spherical data. Geophys. J. R. astr. Soc. 69, 1-13.

Li, X. and R. Jeanloz (1891a) Effect of Iron Content on the Electrical Conductivity of Perovskite and Magnesiowustite Assemblages at Lower Mantle Conditions. J. Geophys. Res. 96, B4, 6113-6120.

Li, X. and R. Jeanloz (1991b) Phase identification and electrical conductivity of an $\mathrm{H}_{2} \mathrm{O}$-bearing silicate assemblage at lower mantle conditions. Nature 350, 332-334.

Mareschal M., W.S. Fyfe, J. Percival and T. Chan (1982) Grain-boundary graphite in Kapuskasing gneisses and implications for lower-crustal conductivity. $\mathrm{Na}$ ture 357, 6380, 674-676.

Marshall, R.T. (1980) Geomagnetic Storm-Time Variations and the Determination of Upper Mantle Conductivity. Ph.D. thesis Exeter University. 
Matsushita, S. (1975) Morphology of Slowly-Varying Geomagnetic External Fields - A Review. Phys. Earth Planet. Int. 10 299-312.

McPherron, R.L. (1979) Magnetospheric Substorms. Rev. Geophys. Space Phys. 17 657-680.

Mosteller F. and J.W. Tukey (1077) Data Analysis and Regression. AddisonWesley Publishing Company.

Nishida, A. (1978) Geomagnetic Diagnosis of the Magnetosphere. Springer Verlag, N.Y.

Parker, R.L. (1970) The Inverse Problem of Electrical Conductivity in the Mantle. Geophys. J. R. astr. Soc. 22, 1-17.

Parker, R.L. (1980) The Inverse Problem of Electromagnetic Induction: Existence and Construction of Solutions Based on Incomplete Data. J. Geophys. Res., 85, 44214425.

Parker, R.L. (1982) The existence of a Region Innaccessible to Magnetotelluric Sounding. Geophys. J. R. astr. Soc., 68 165-170.

Parker, R.L. (1983) The Magnetotelluric Inverse Problem. Geophysical Surveys, 6, 5-25.

Parker, R.L. and K.A. Whaler (1981) Numerical Methods for Establishing Solutions to the Inverse Problem of Electromagnetic Induction. J. Geophys. Res., 86 B10, 9574-9584.

Parkinson, W.D. and V.R.S. Hutton (1989) The Electrical Conductivity of the Earth. In: Jacobs J.A. (ed.) Geomagnetism. Academic Press, London.

Pecova, J., K. Pec and O. Praus (1980) Remarks on Spatial Distribution of Long Period Geomagnetic Variations in the Geomagnetic Field over European Area. In: U. Schmucker (ed.) Electromagnetic Induction in the Earth and Moon. Academic Publications, Japan, 171-186. 
Petersons, H.F. and R.S. Anderssen (1990) On the Spherical Symmetry of the Electrical Conductivity of the Earth's Mantle. J. Geomag. Geoelectr. 42, 1309-1324.

Report UAG-82 (1985) Combined International Catalog of Geomagnetic Data. U.S. National Geophysical Data Center, Boulder, Colorado.

Rikitake T. and S. Sato (1957) The geomagnetic Dst field of the magnetic storm on June 18-19, 1936. Bull. Earthquake Res. Inst. Tokyo Univ., 357.

Rikitake T., (1866) Electromagnetism and the Earth's Interior. Elsevier, Amsterdam, 221-226.

Roberts, R.G. (1982) The Electromagnetic Response of the Earth and Upper Mantle Electrical Conductivity. Ph.D. thesis Lancaster University.

Roberts, R.G. (1984) The long-period electromagnetic response of the Earth. Geophys. J. R. astr. Soc., 78, 547-572.

Roberts, R.G. (1986) Global Electromagnetic Induction. Surveys in Geophysics, 8, 339-374.

Rokityansky, I.I. (1982) Geoelectromagnetic Investigation of the Earth's Crust and Mantle. Springer-Verlag, Berlin, Heidelberg.

Russell, B. (1946) History of Western Philosophy. George Allen and Unwin Ltd.

Schmucker U. and J. Jankowski (1872) Geomagnetic Induction Studies and the Electrical State of the Upper Mantle. In: Ritsema, A.R. (ed.) Developments in Geophysics 4, The Upper Mantle. Elsevier Publishing Company.

Schultz, A. and J.C. Larsen (1983) Analysis of Zonal Field Morphology and Data Quality for a Global Set of Magnetic Observatory Daily Mean Values. J. Geomag. Geoelectr. 35, 835-846.

Schultz, A. and J.C. Larsen (1887) On the electrical conductivity of the midmantle-I. Calculation of equivalent scalar magnetotelluric response functions. Geophys. J. R. astr. Soc. 88, 733-761. 
Schultz, A. and J. Larsen (1990) On the electrical conductivity of the mid-mantle: II. Delineation of heterogeneity by application of extremal inverse solutions. Geophysical Journal International 101, 565-580.

Schultz, A. (1990) On the vertical gradient and associated heterogeneity in mantle electrical conductivity. Phys. Earth Planet. Int. 64, 68-86.

Shure, L., K. Whaler, D. Gubbins and B. Hobbs (1983) Physical constraints for the analysis of the geomagnetic secular variation. Phys. Earth Planet. Int. 32 114-131.

Singleton, (1968) Mixed-Radix Fast Fourier Transform Algorithm. Stanford Research Institute.

Stewart, M.P. (1980) Computation of a Gravimetric Geoid for the British Isles: An assement of Fourier and Classical Techniques. Ph.D. thesis Edinburgh University.

Stratton, J.A. (1941) Electromagnetic Theory. McGraw-Hill, New York.

Sugiura, M. (1964) Hourly values of equatorial Dst for the IGY. Ann. International Geophys. Year 35 9-45.

Thompson, R. and R.M. Clark (1982) A robust least-squares Gondwanan apparent polar wander path and the question of palaeomagnetic assessment of Gondwanan reconstructions. Earth and Planet. Sci. Lett. 57 152-158.

Thomson, D.J. (1977) Spectrum estimation techniques for characterization and development of WT4 waveguide, I. Bell Syst. Tech. J. 56 1769-1815.

Thomson, D.J. (1982) Spectrum Estimation and Harmonic Analysis. Proceedings of the IEEE 70 1055-1096.

Weidelt, P. (1972) The Inverse Problem of Geomagnetic Induction. Zeitschrift fur Geophysik, 38, 257-289.

Wood, B.J. and L. Nell (1991) High-temperature electrical conductivity of the lower-mantle phase $(\mathrm{Mg}, \mathrm{Fe})$ O. Nature $351,309-311$. 


\section{General Reference}

Parkinson, W.D. (1983) Introduction to Geomagnetism. Scottish Academic Press Ltd. 
Appendix A

Additional Figures and Tables 
Figure A.1. European Observatories.

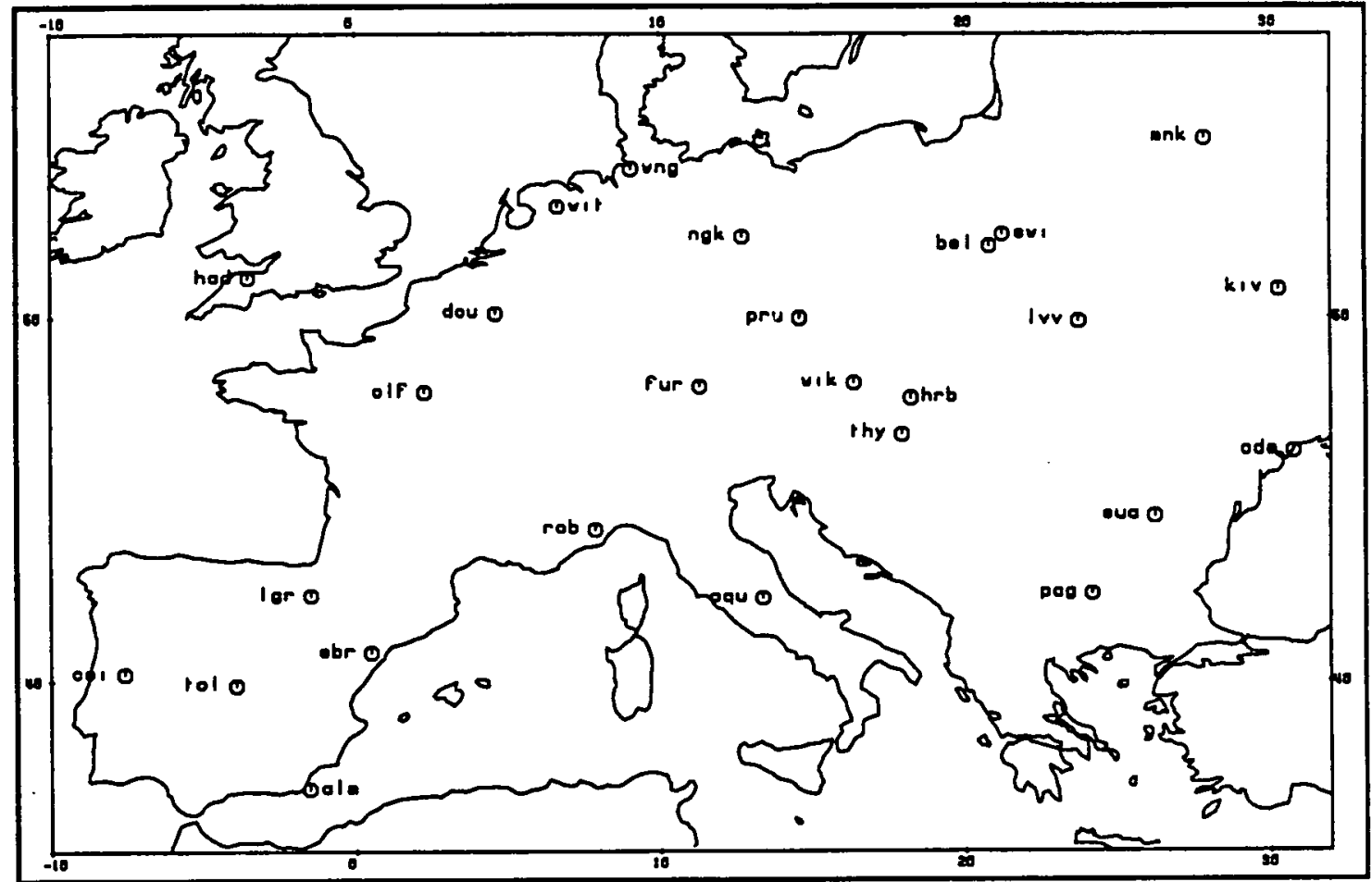

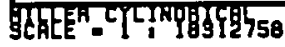


Figure A.2. A Selection of Original Magnetograms for Storm 4. Top trace $X$ Component with $Y$ and then $Z$ below.
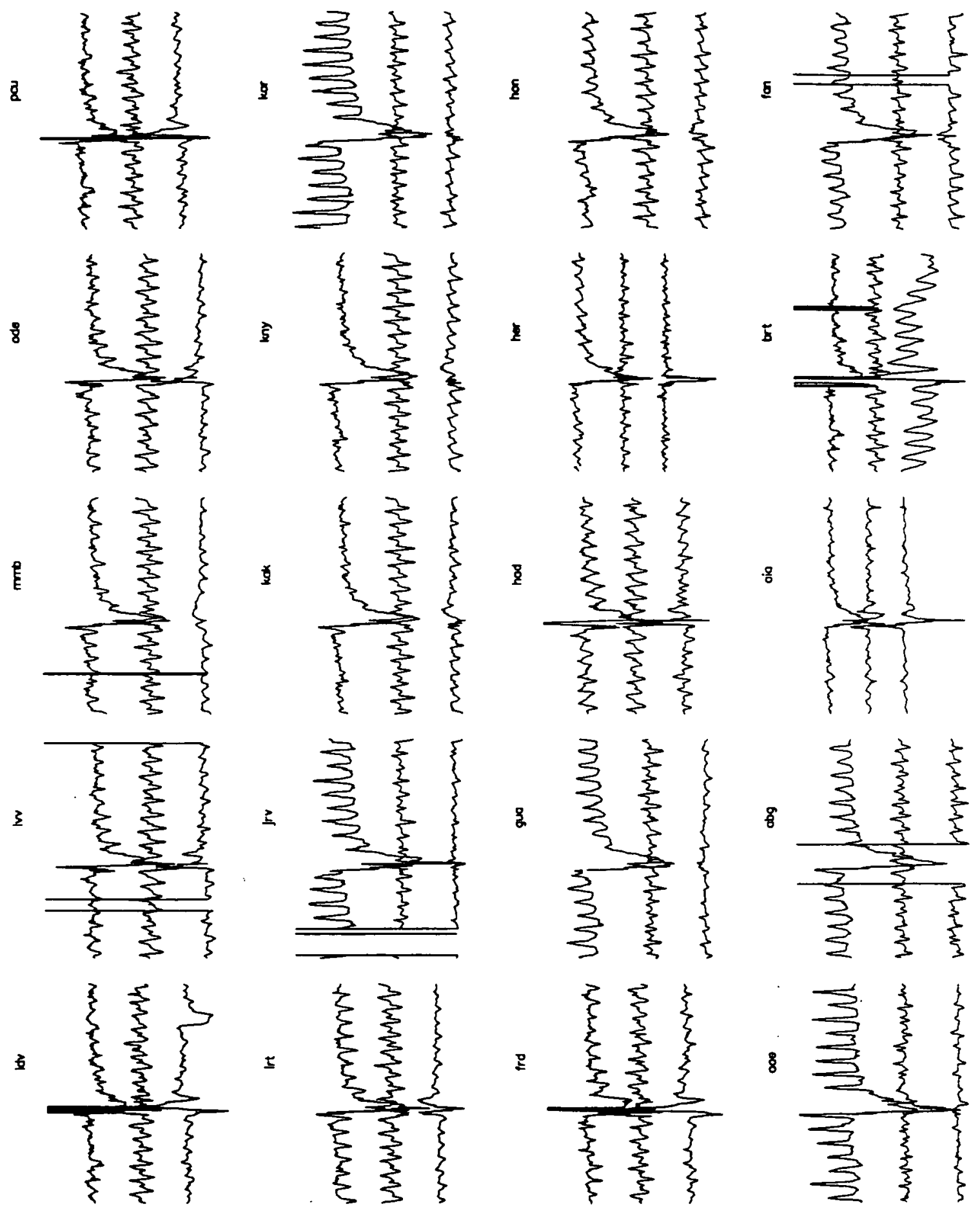
Figure A.3. A Selection of Magnetograms for Storm 4 after having Removed the Daily Variation and Interpolated for Missing Data. Top trace $X$ Component with $Y$ and then $Z$ below.
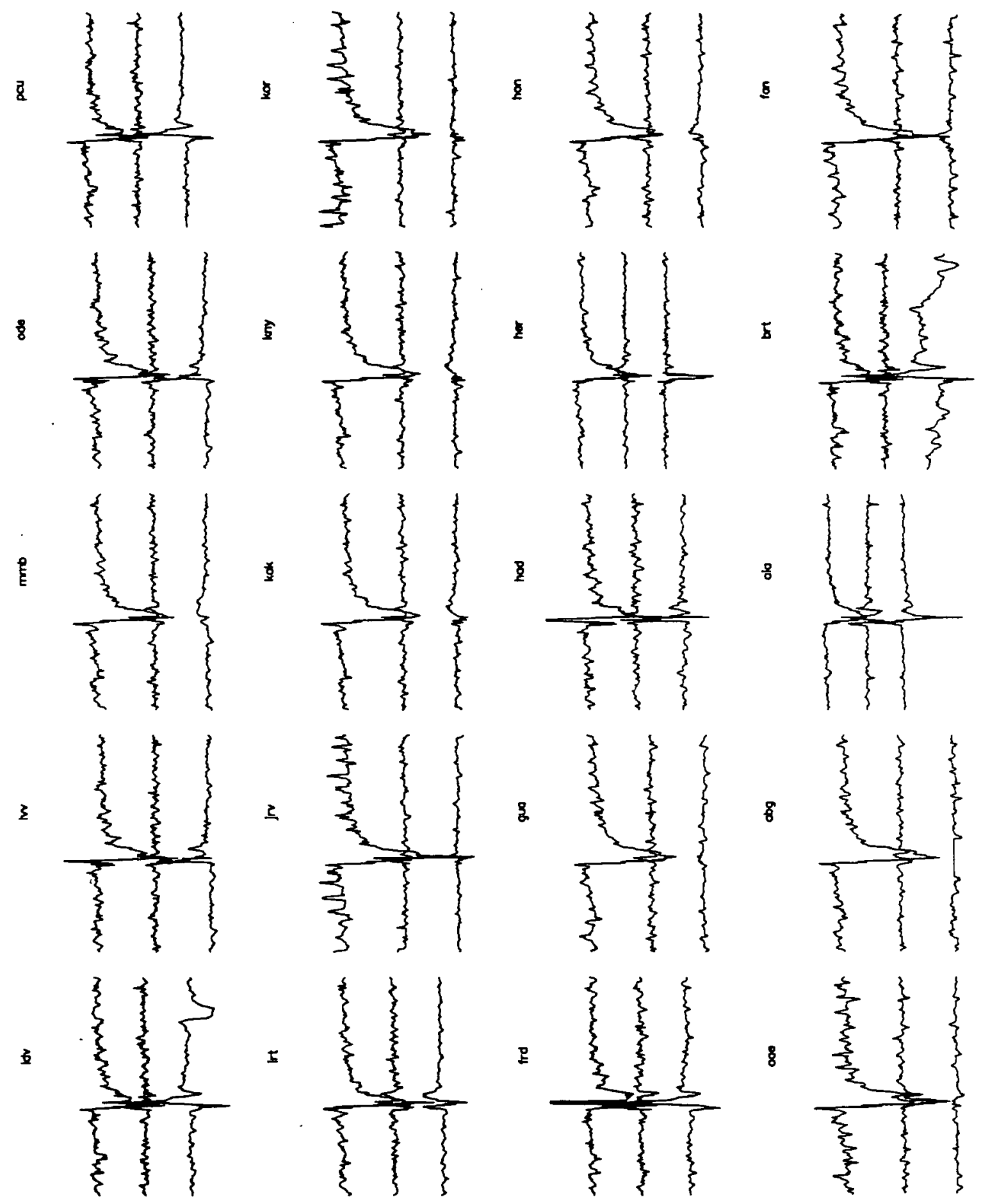

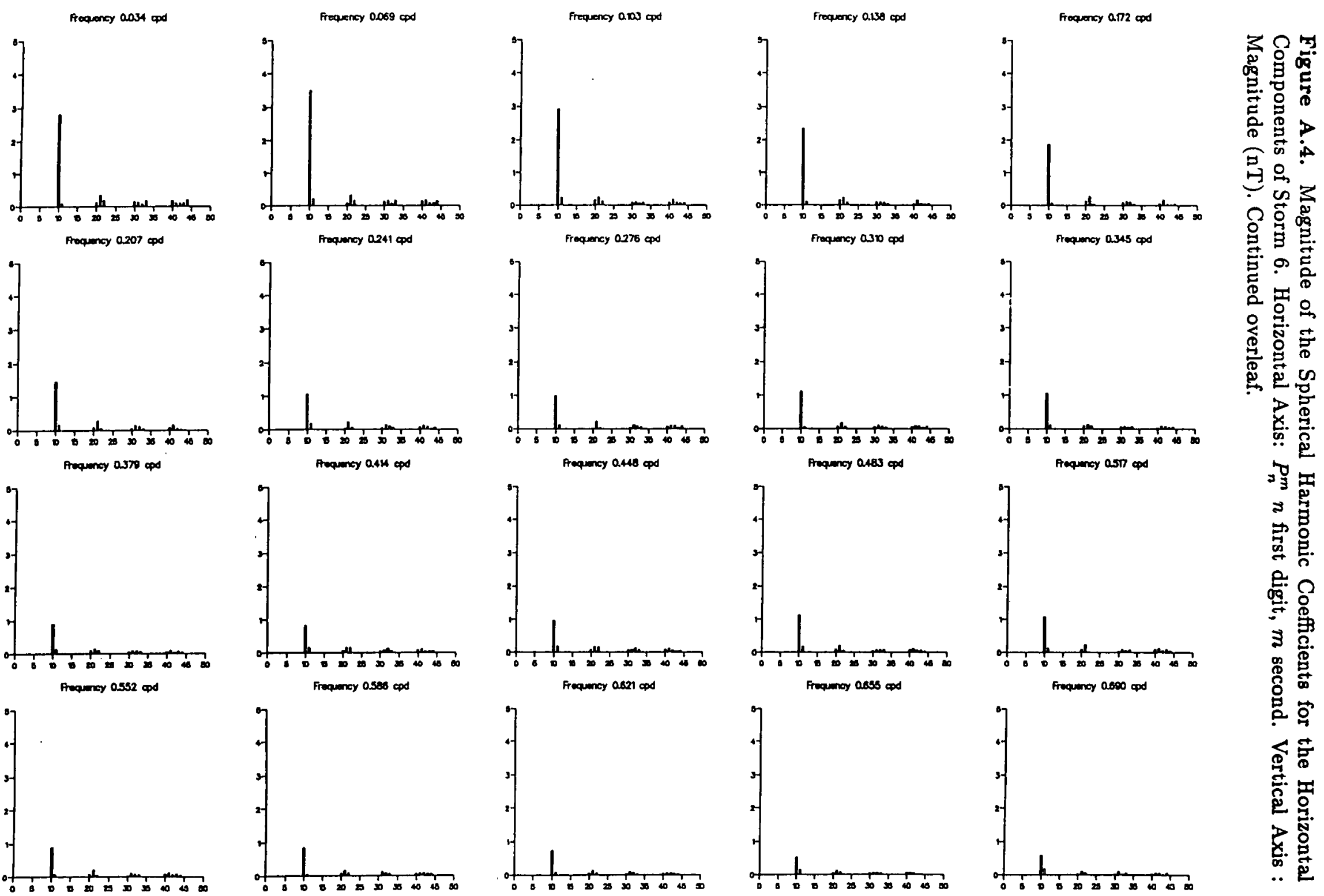

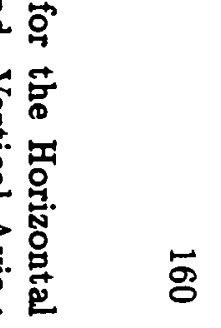




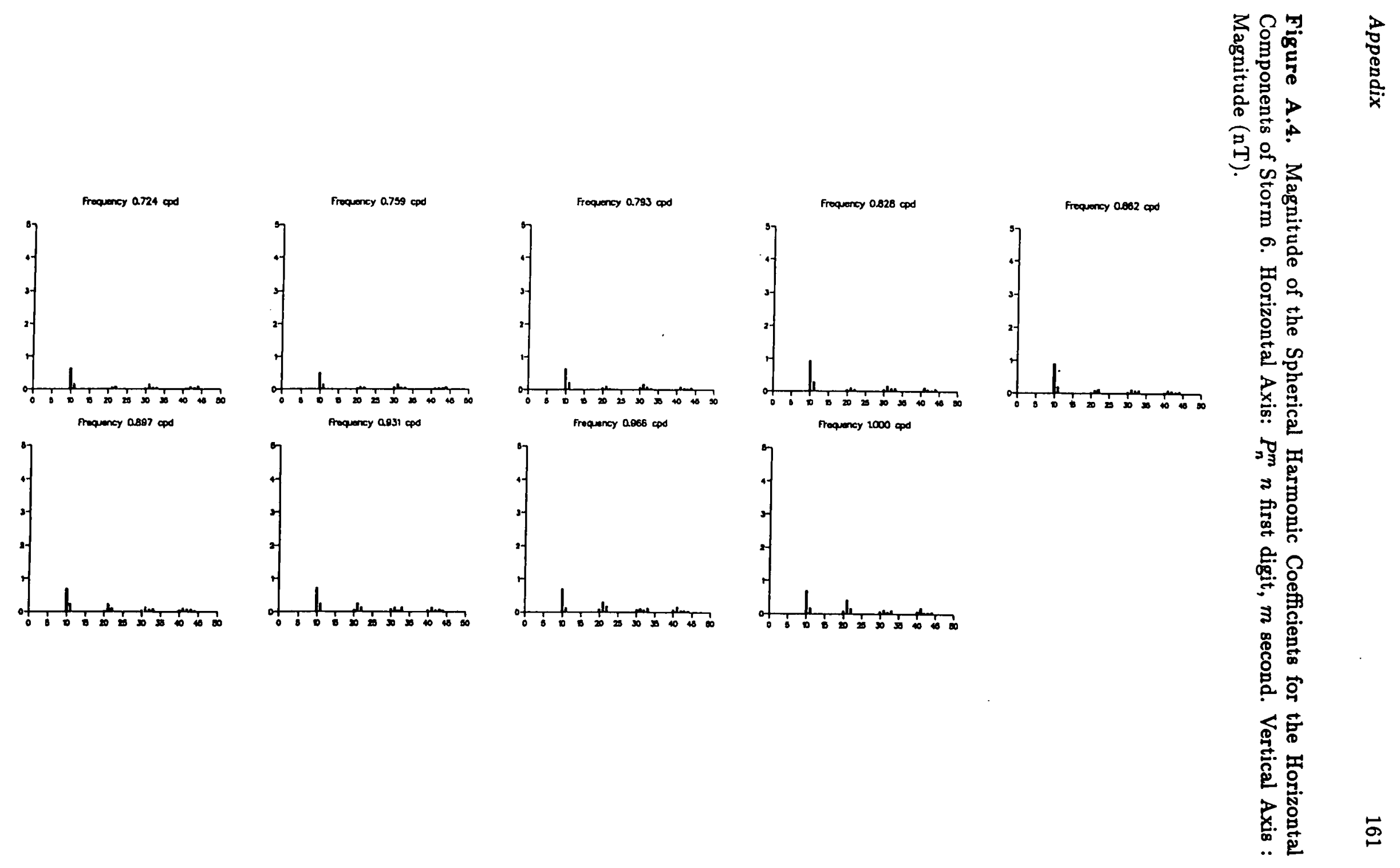



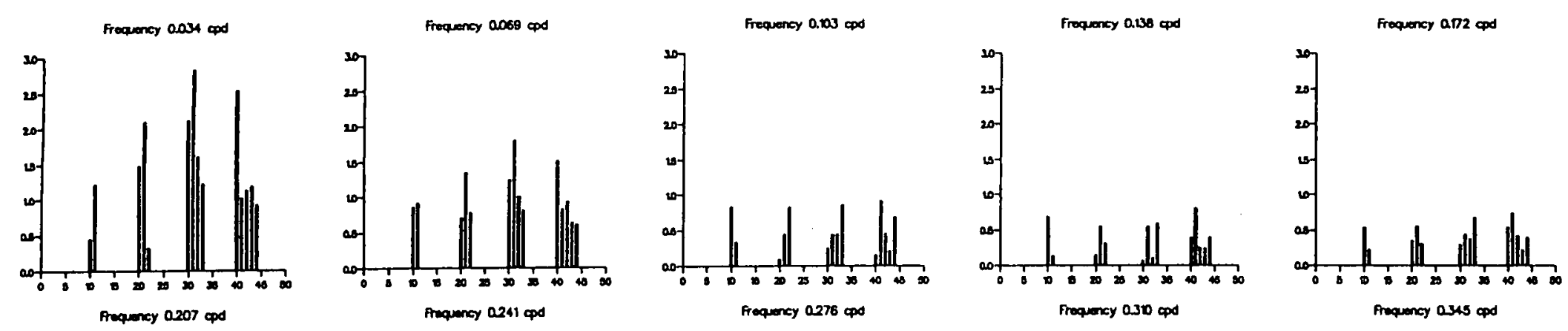

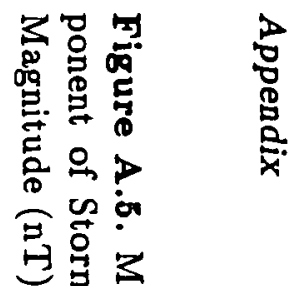
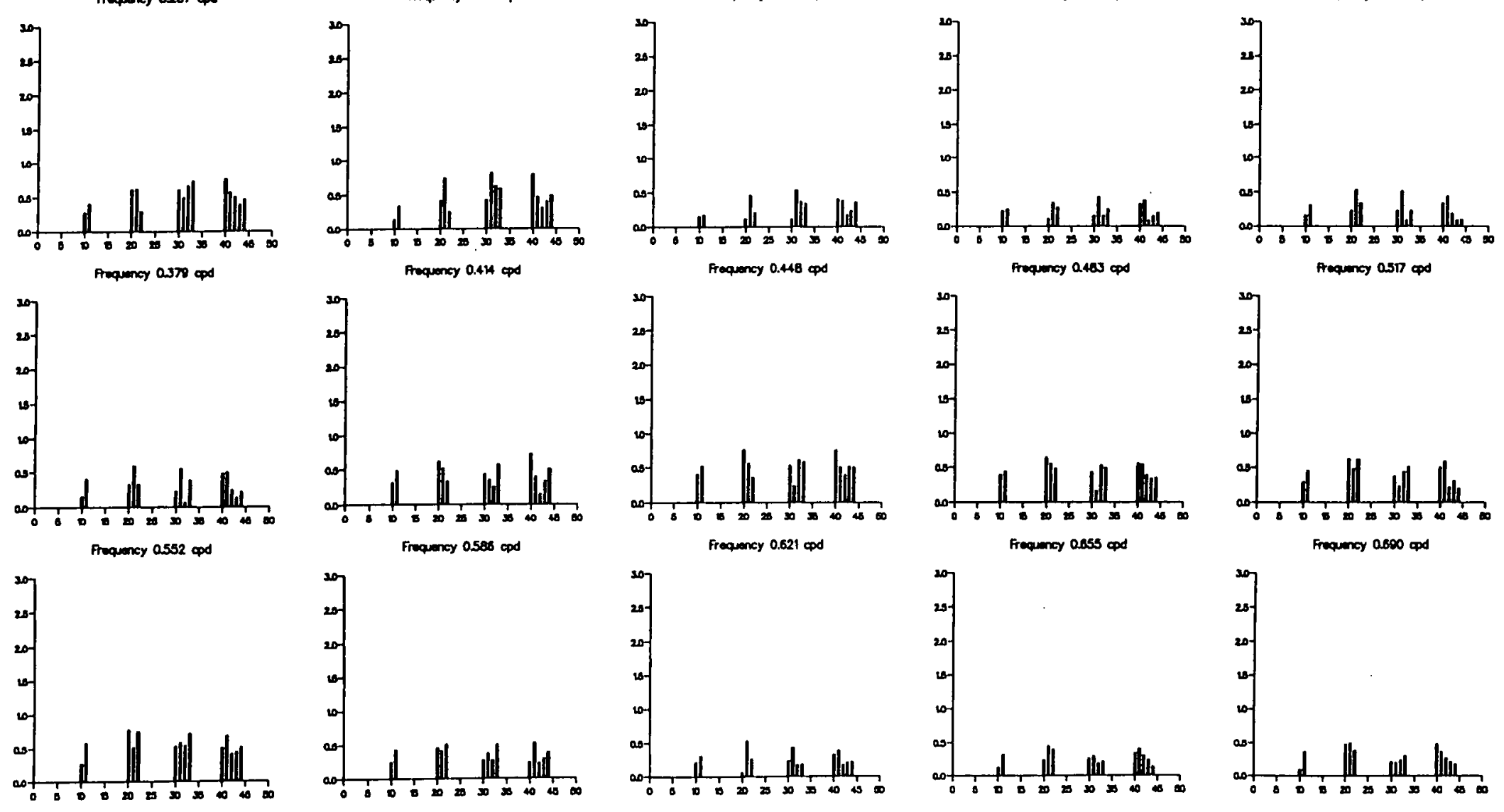

Оᄋํํㅇ.

홉 정 흥

吝突品

总宽

帘

突总

ต․

肯

ว

局㐘.

氮.

इ

品

옹

.

(

突.

용. 


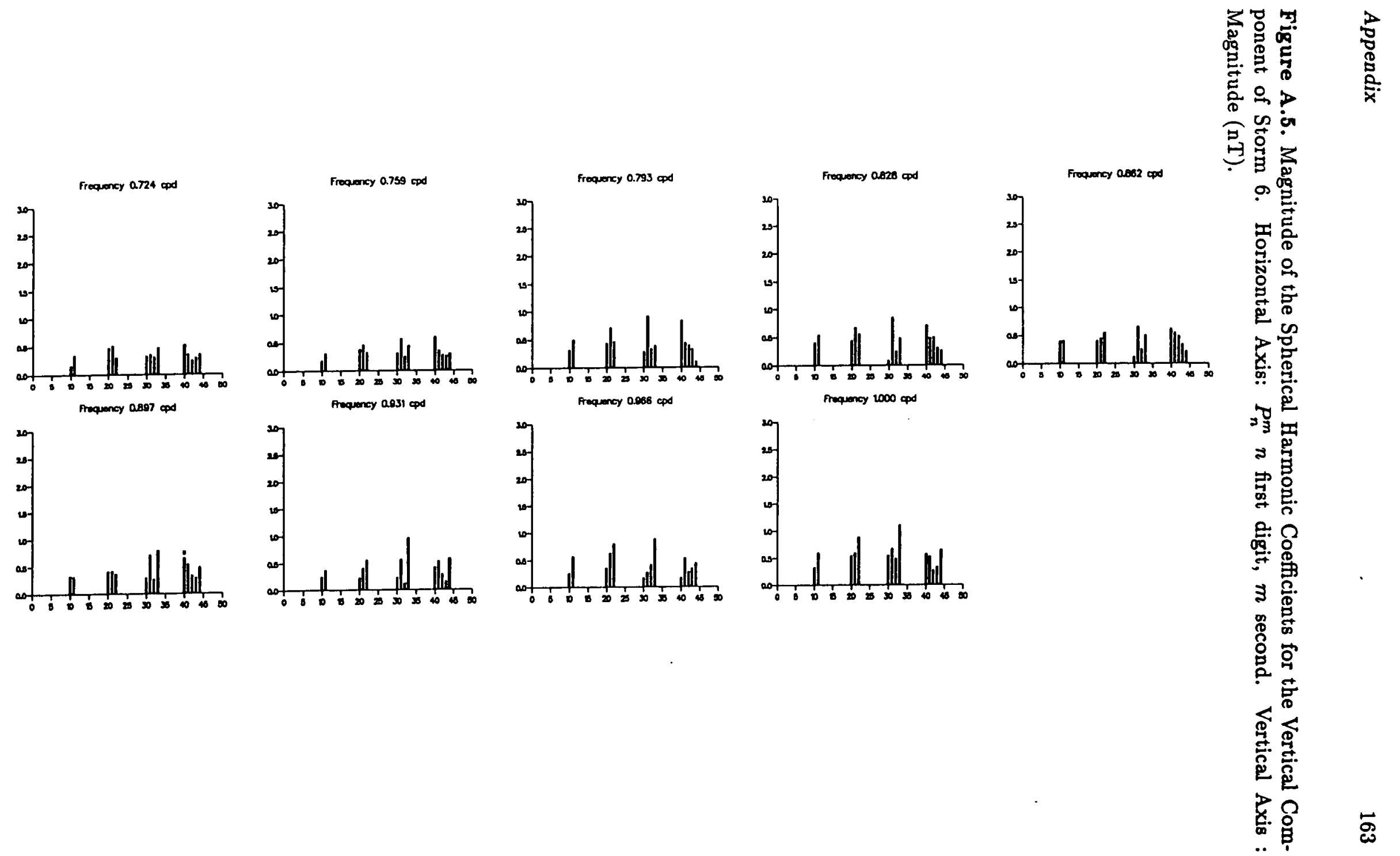




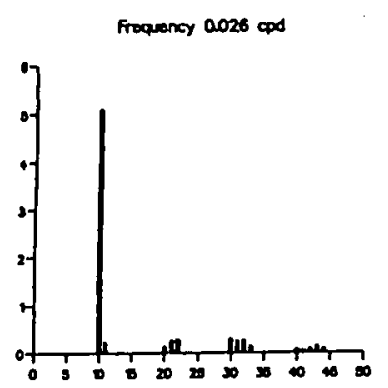

frequency 0.54 cod
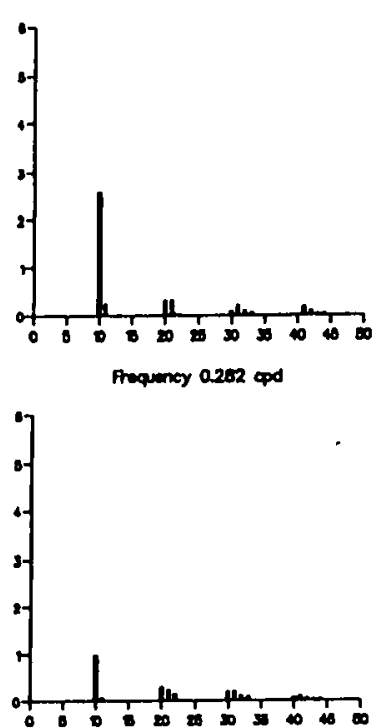

froamer 0.40 cod

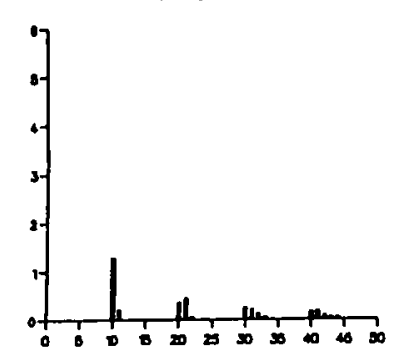

fropuncy 0.051 epd

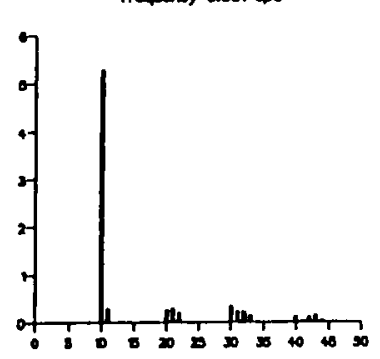

Arequency a.mo opd
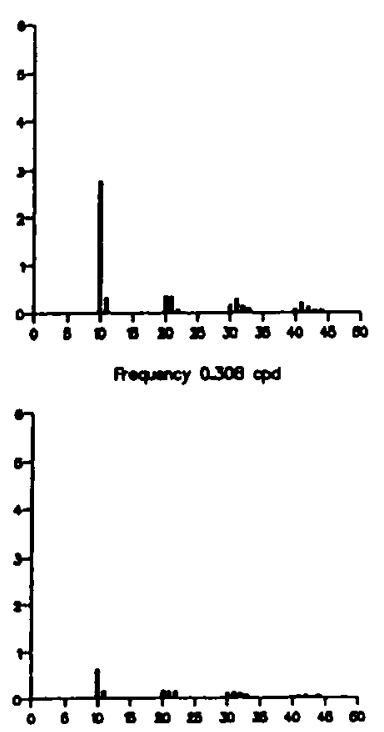

froguncy 0.436 od

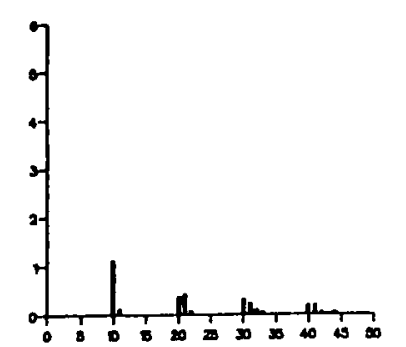

Frequancy 0.07 cod

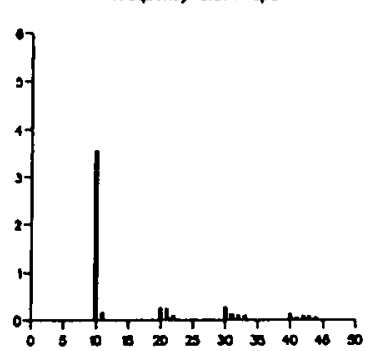

Froquency a.20s cpd

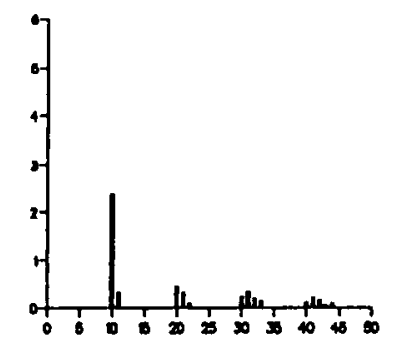

Fropeney $0.30 \mathrm{cos}$
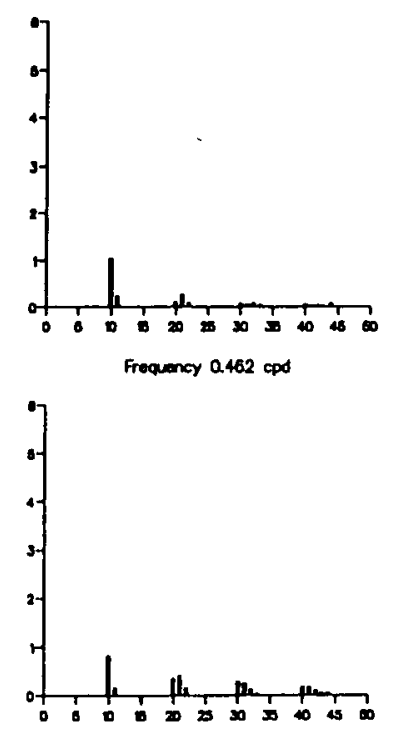

Froquency 0.03 opd
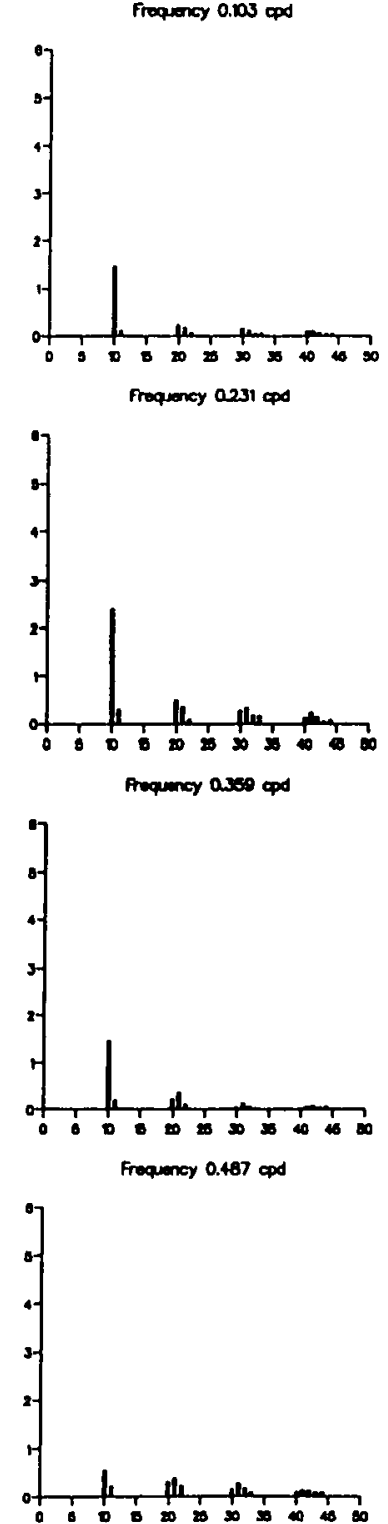

Froquany 0.08 ad

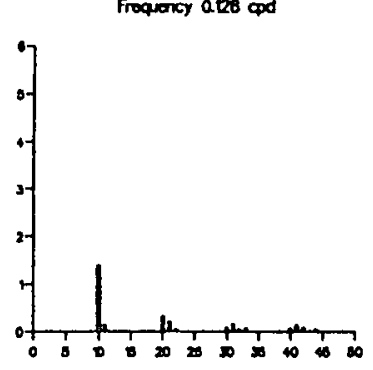

Proquency 0256 cos

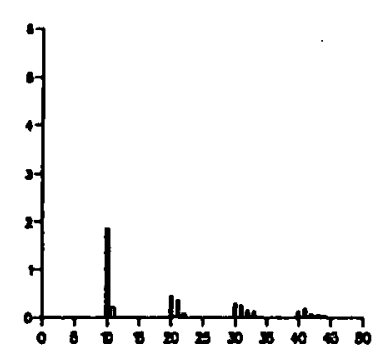

fraquncy ases ed
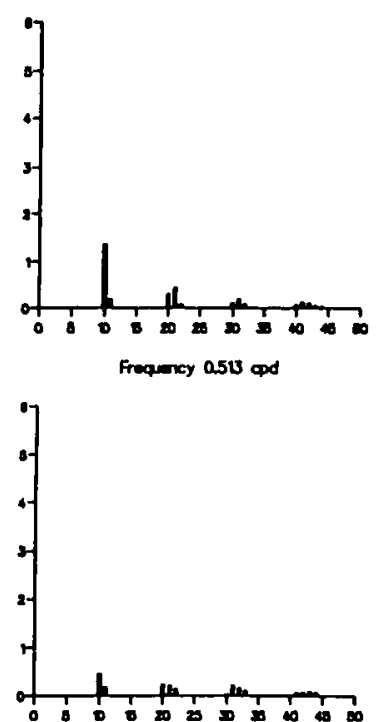

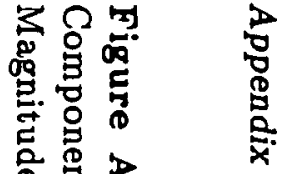

官点

봉.

$\div$ 雪

ᄋํํㄱ

㕺它它

国。

엉웅

害宫

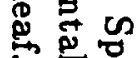

要.

ํ.ฺํ.

语畟

$\rightarrow \frac{\mathrm{B}}{\mathrm{B}}$

경영.

昰.

䒹

จ

总 㤩

昌熍

通穷

옹

종. 


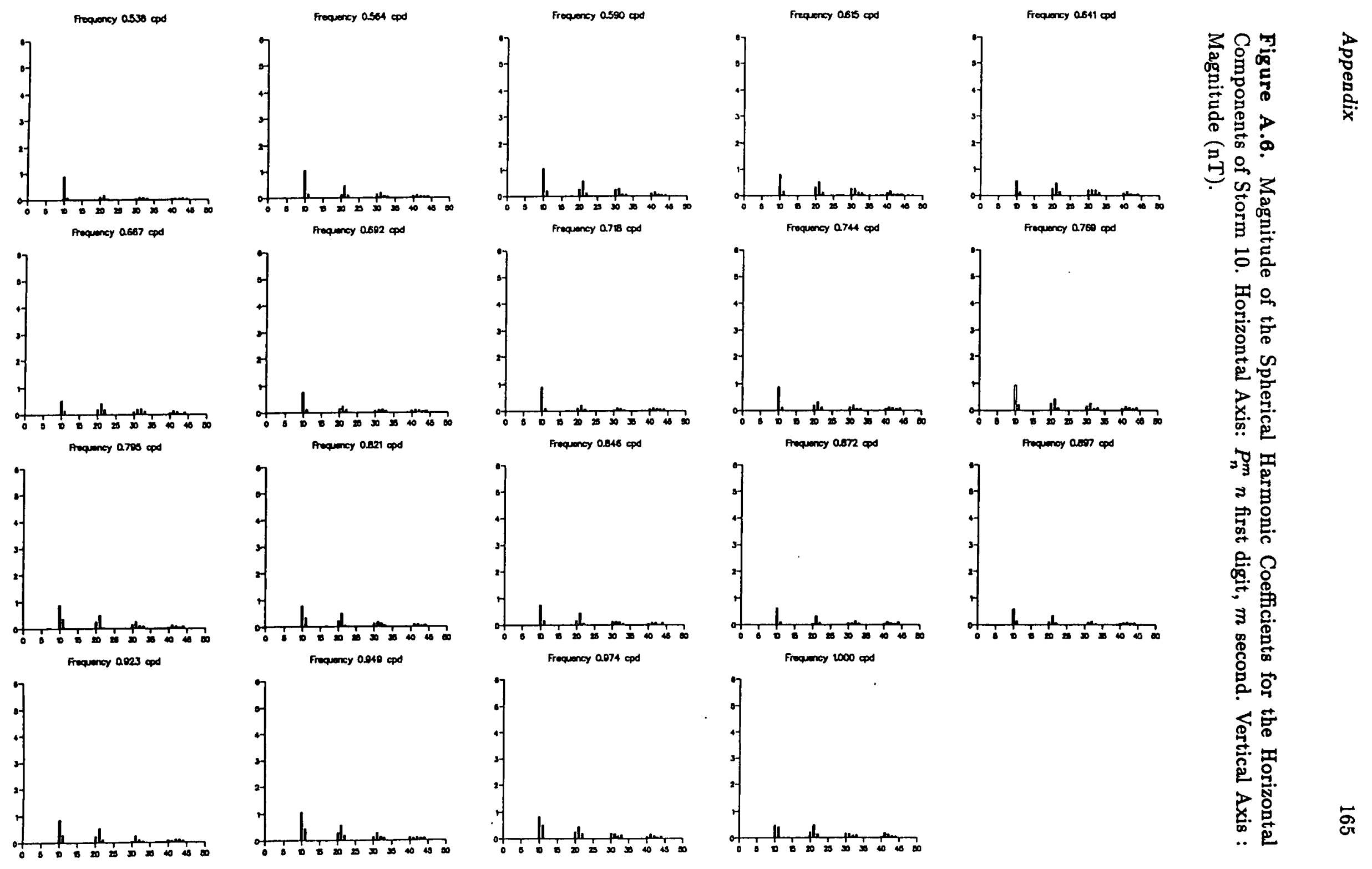



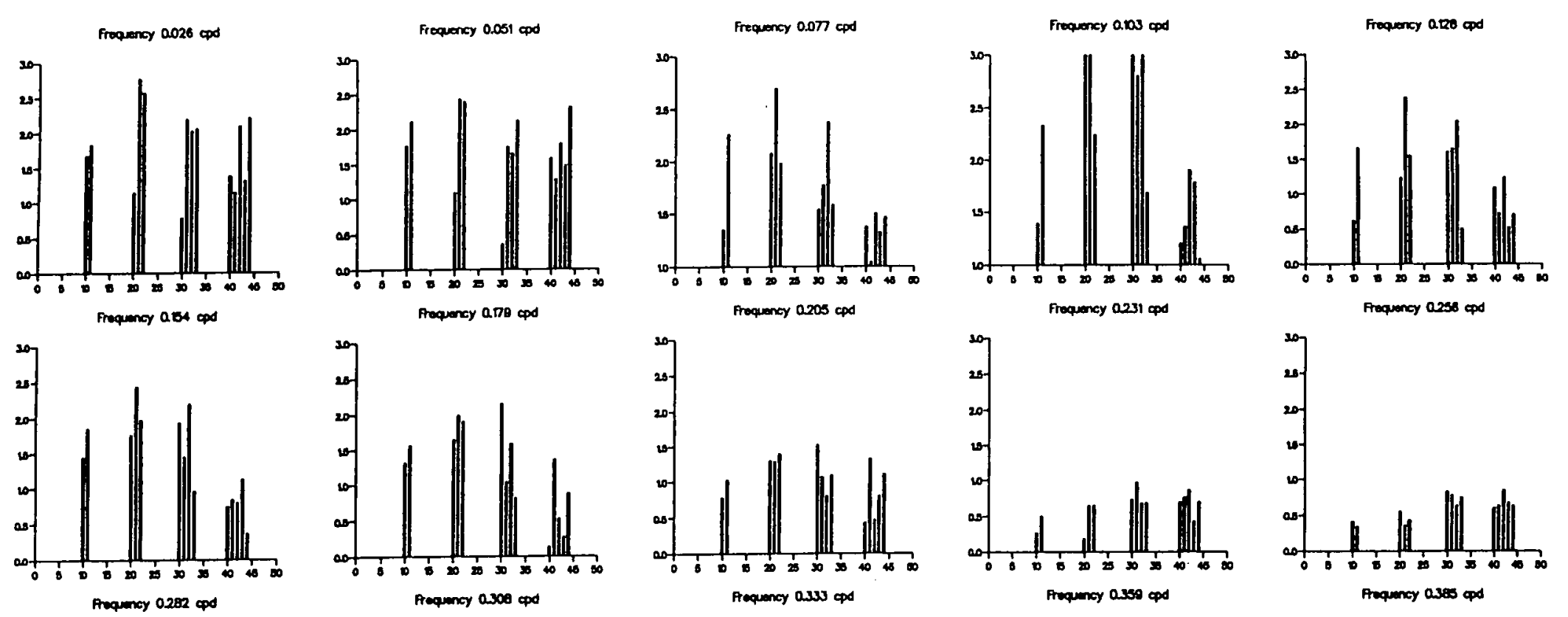

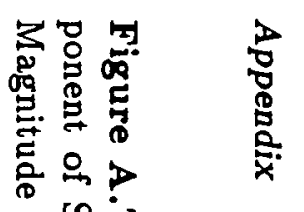
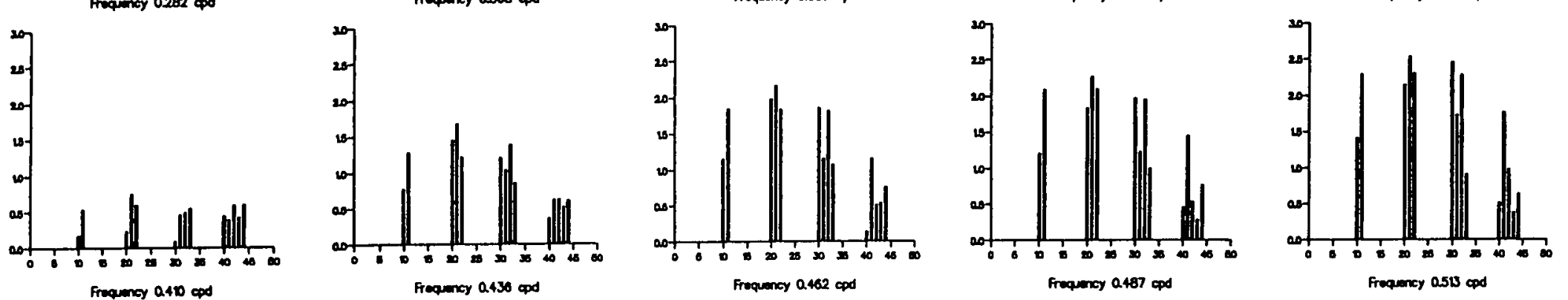

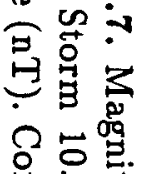

氜

옹용

응

进芭

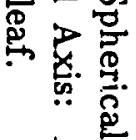

谓察



费号.

용요

इ

\%

옴
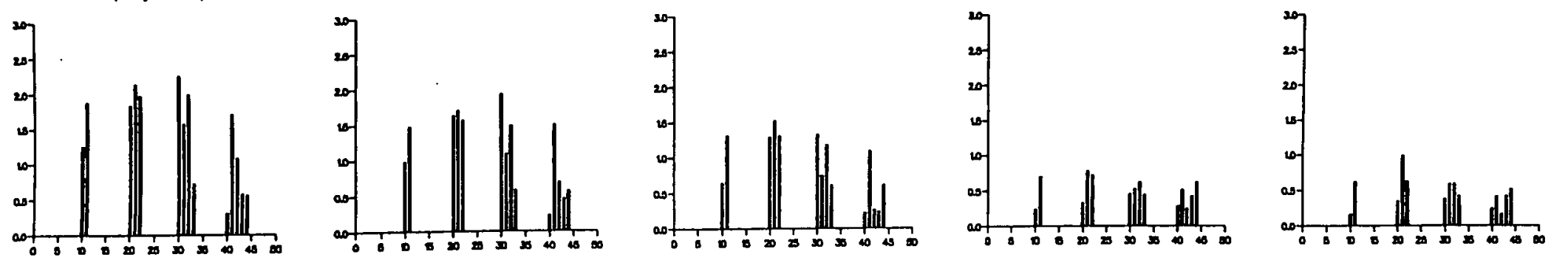

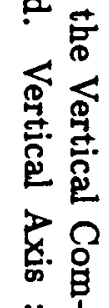



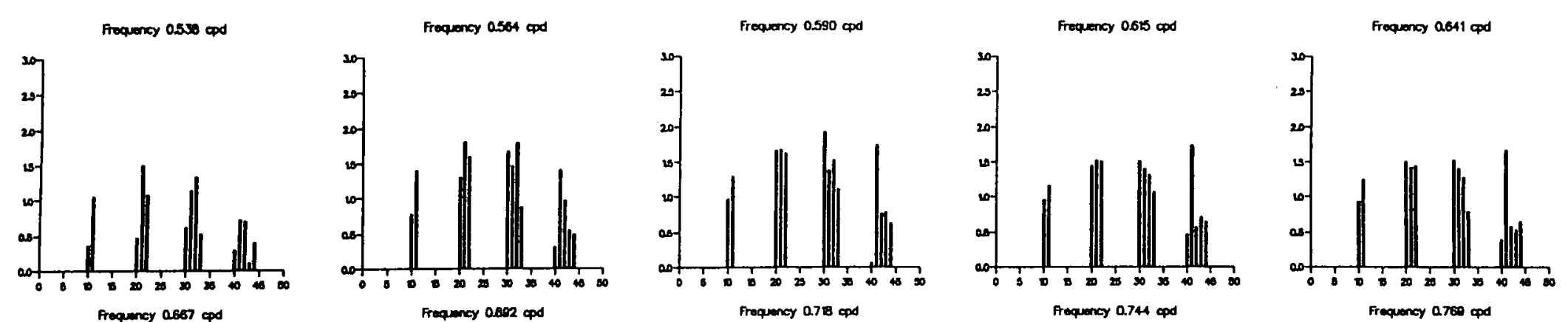

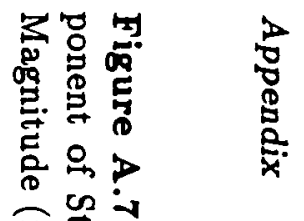
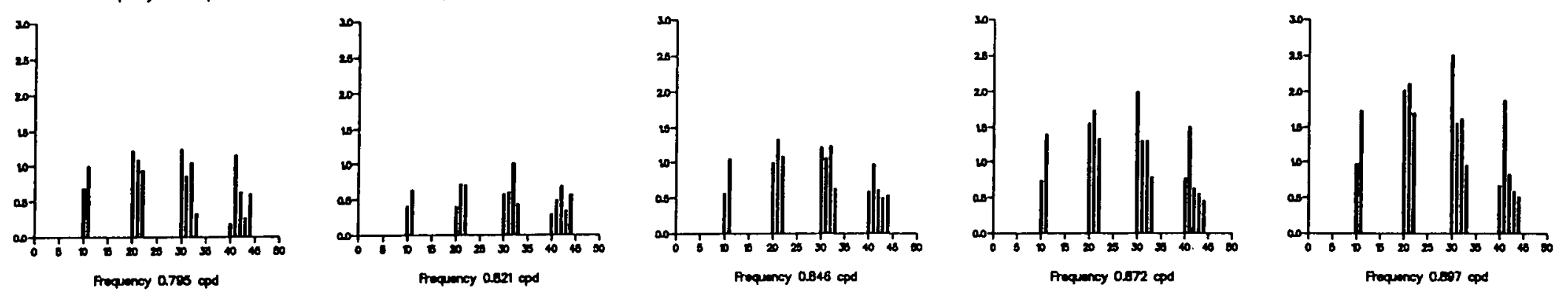

छ 벅영

놀.

四

옹응

宥

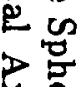

它 哓
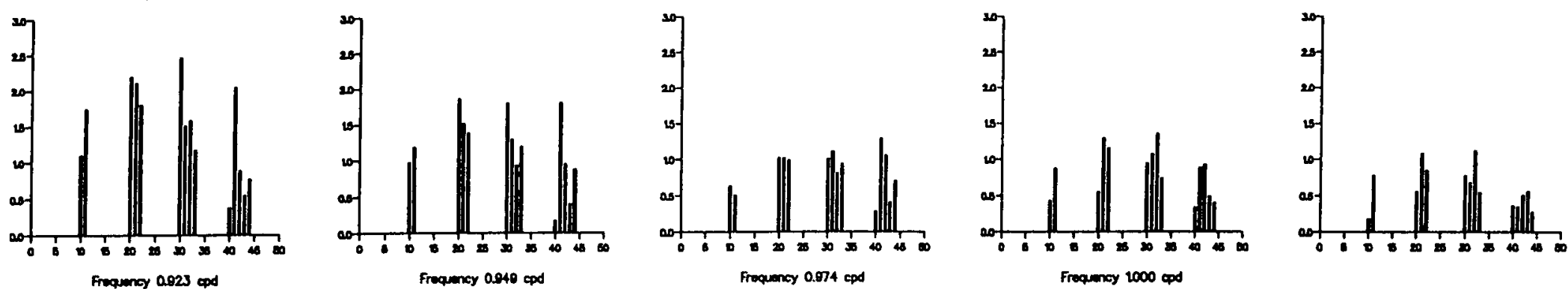

ว

哥 을.

응 ?

买

怘

官 寈

Froquney 1000 od
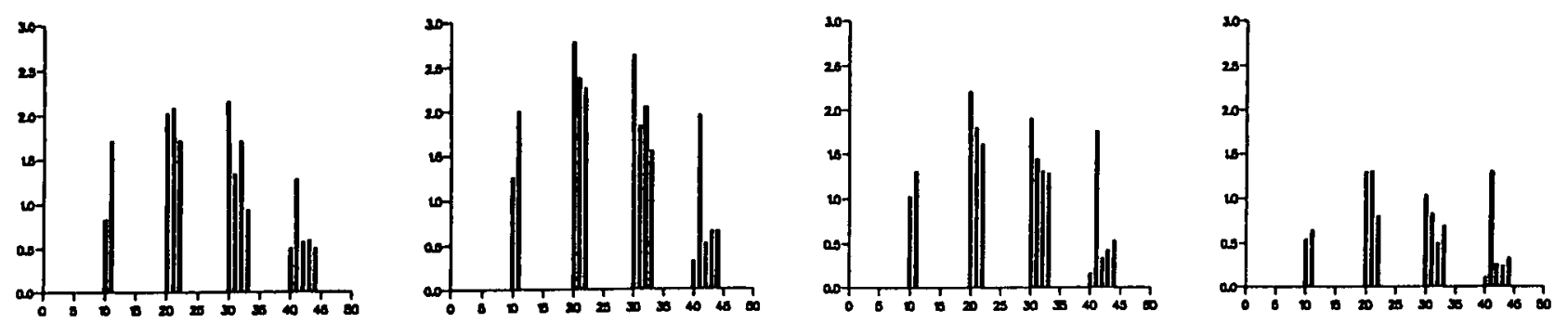

官 


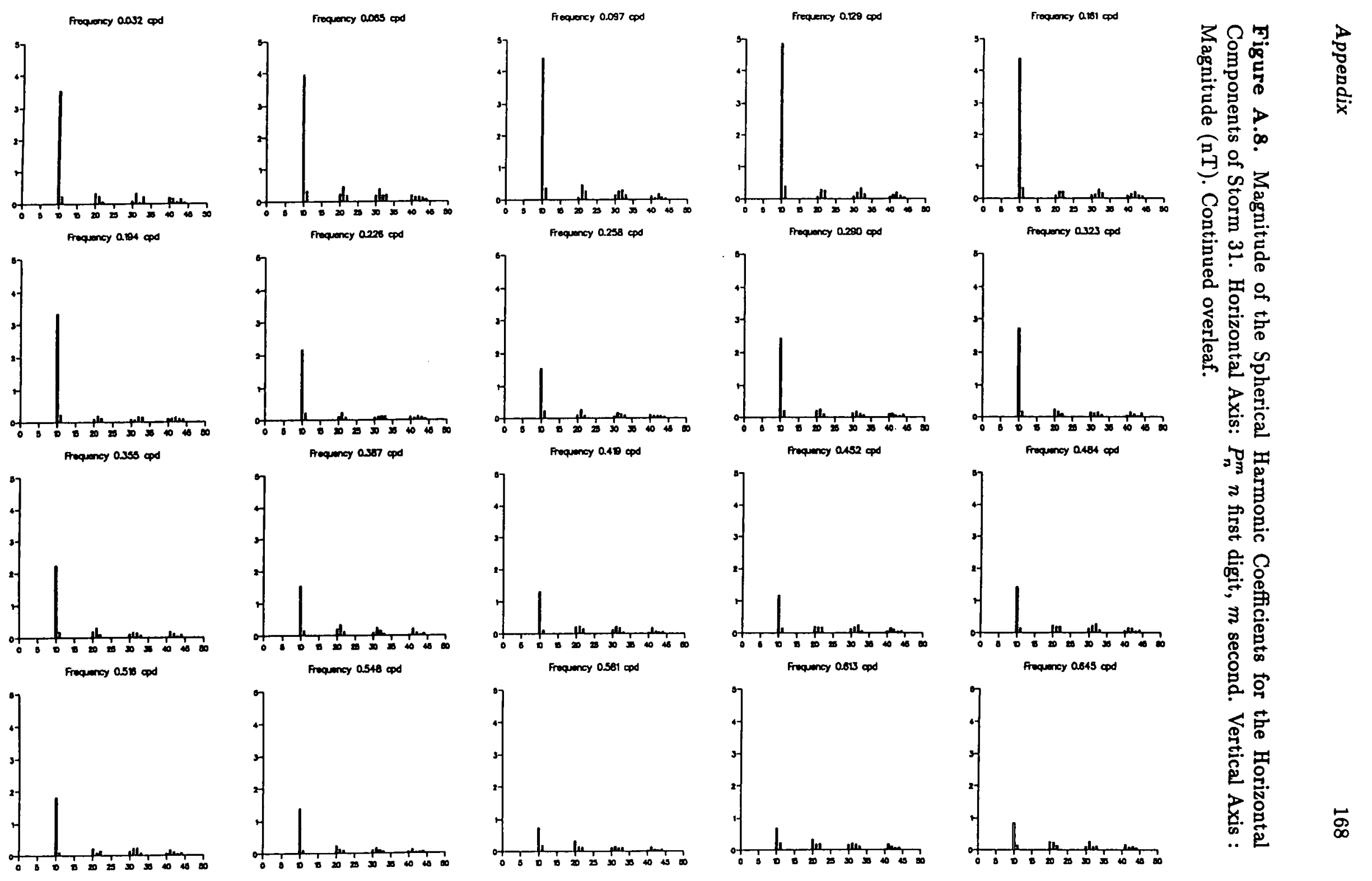



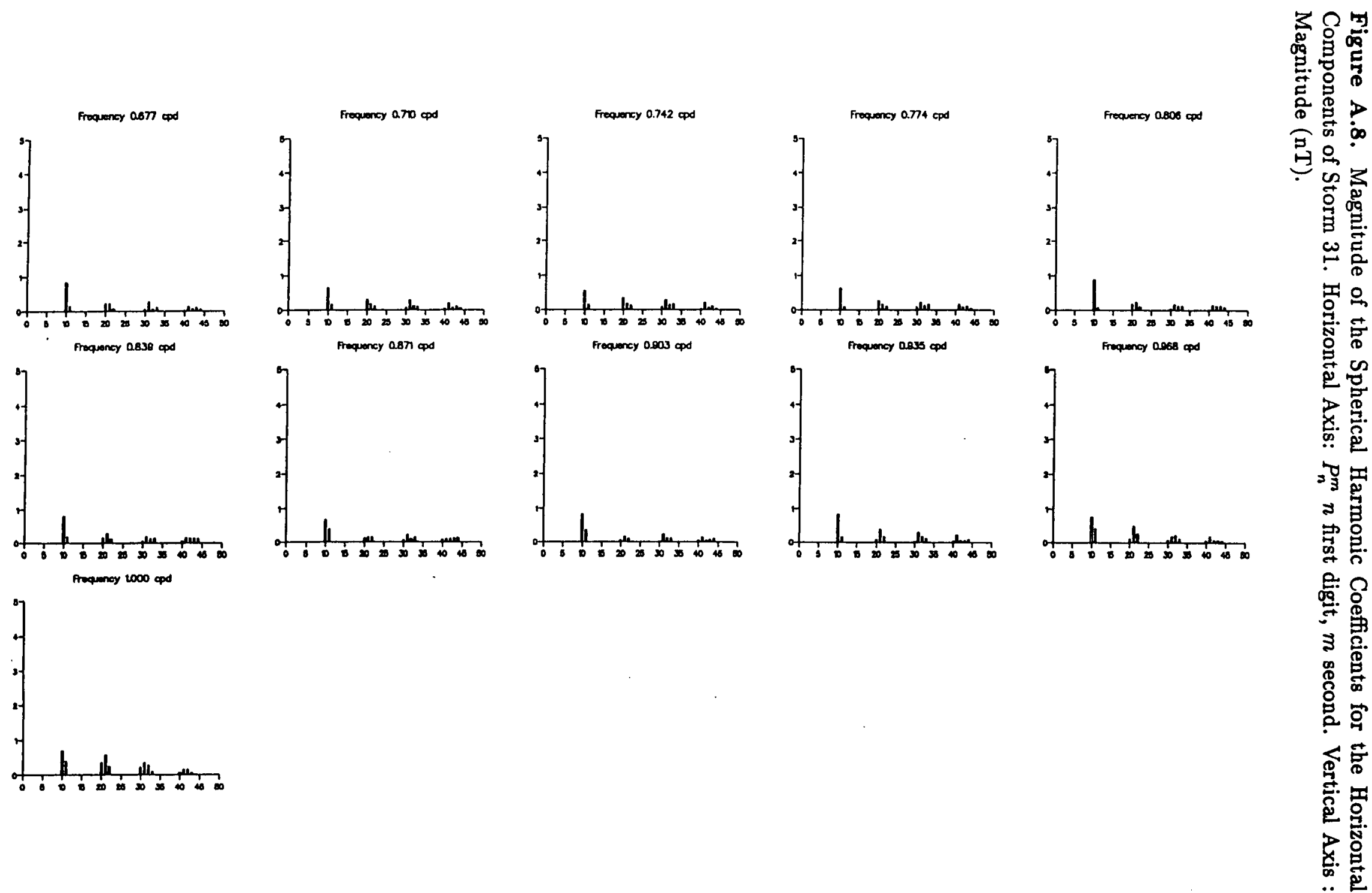

\begin{tabular}{l}
3 \\
8 \\
8 \\
0 \\
0 \\
0 \\
\hdashline
\end{tabular}

Aragency 1000 opd

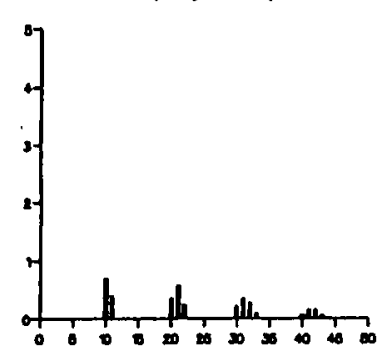



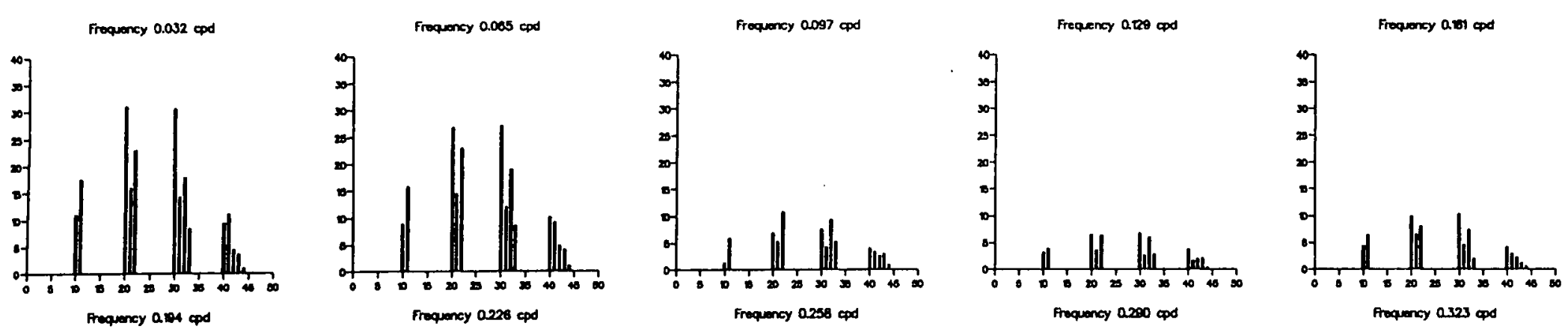

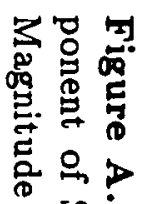

唡过

부ㅇㅛㅛ ᄋํํ릴.
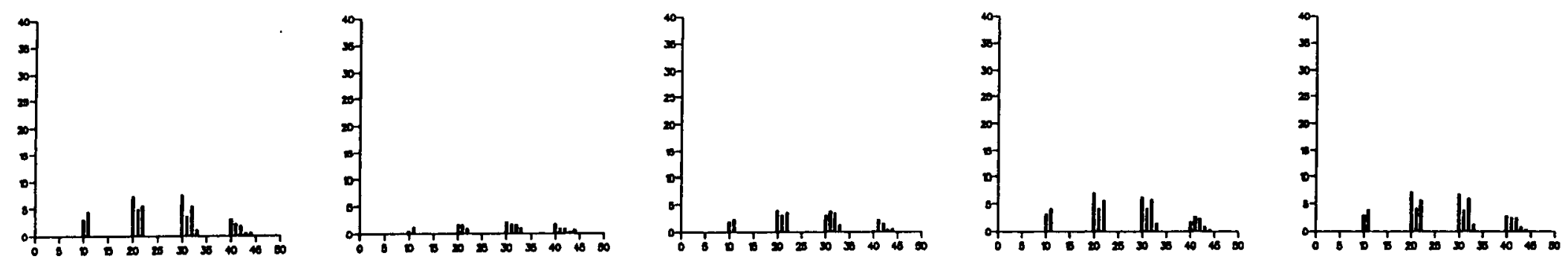

돌

응. 웅

음

可芭 密宫

魚

Aroguncy 0.40 apd

Aropuncy a.452 opd
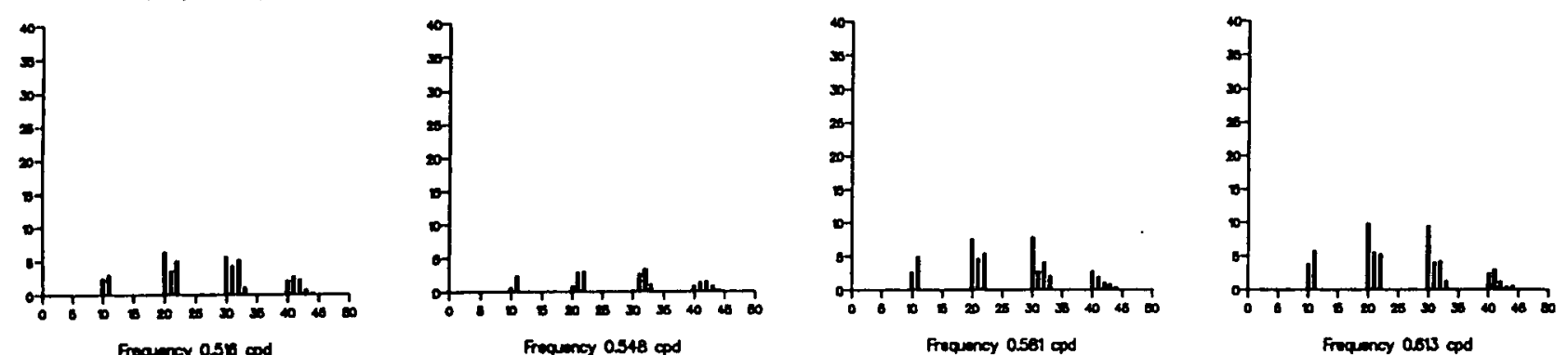

Aroaney 0.404 opd

影

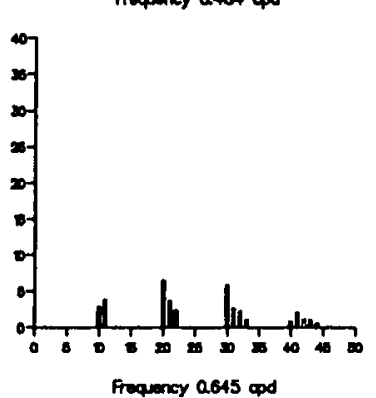

$\approx$

공용.

응

3 ㄱ.

宽

ठั

是
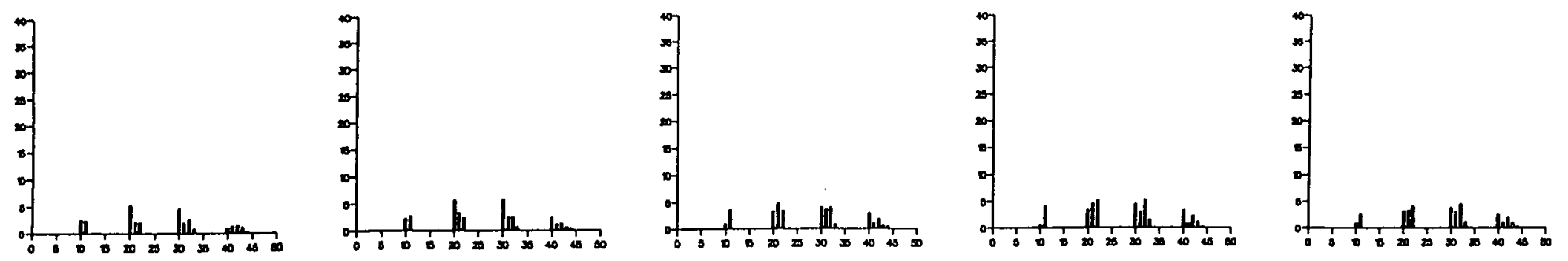

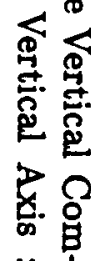



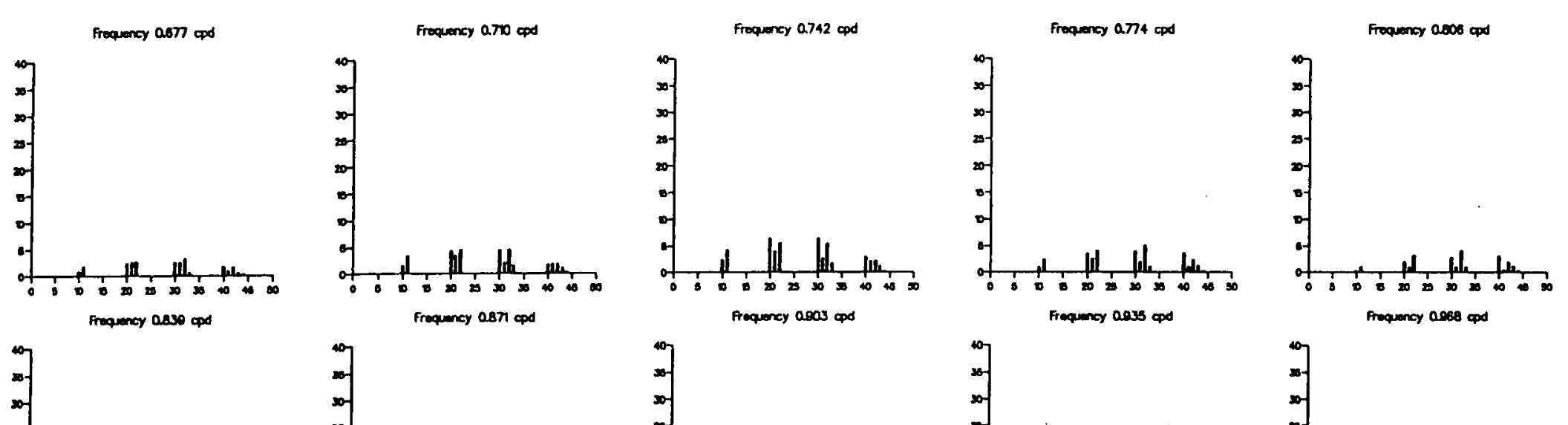

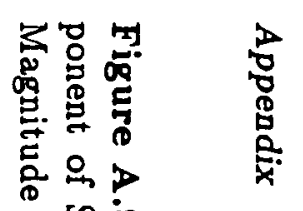
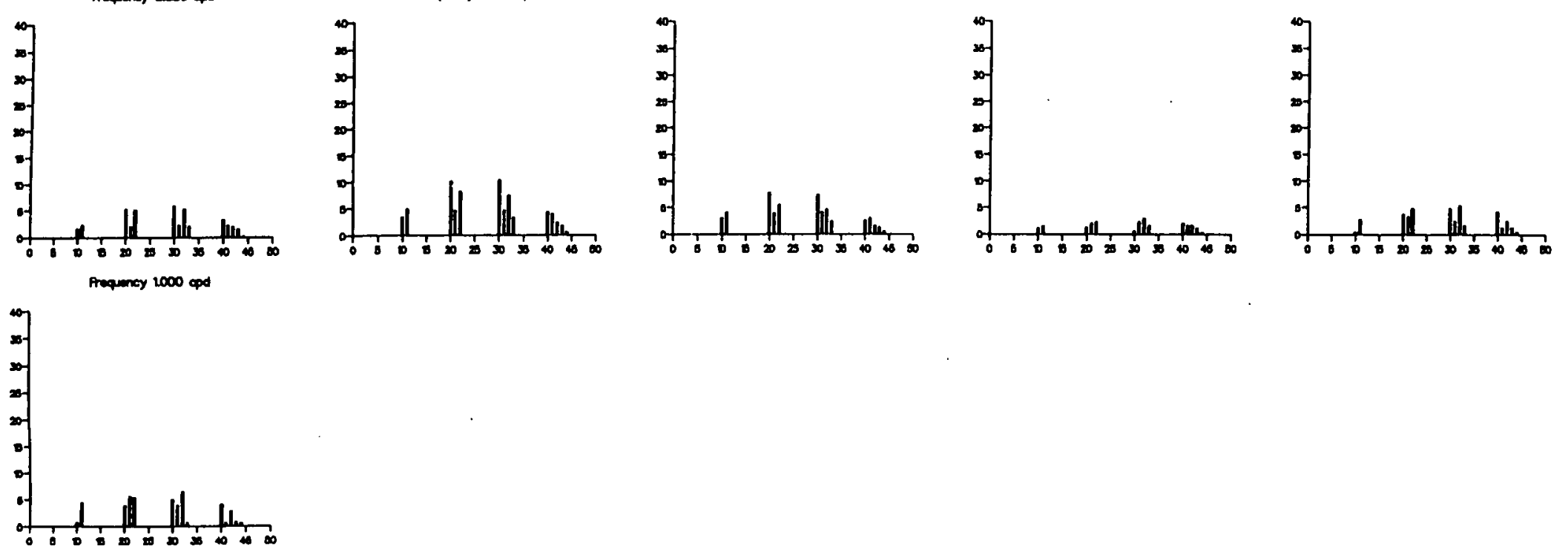

象

హ융

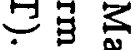

닐

的

옹요

节

边

它

㐭.

空

จ

究 党.

응

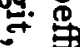

จ

品

实

유

本

楁

客。 
Table A.1. The Average $P_{1}^{0}$ Response in terms of $c$. The error $\delta$ was derived from the jackknife standard errors on $Q$.

\begin{tabular}{|r|r|r|r|r|r|r|}
\hline \multicolumn{2}{|l|}{$\begin{array}{l}\text { Frequency } \\
\text { Band }\end{array}$} & \multicolumn{2}{|c|}{ Band Midpoint } & \multicolumn{2}{|c|}{ Real Part of $c$} & \multicolumn{2}{|c|}{ Imag Part of $c$} \\
\cline { 2 - 7 } & cpd & log(cpd) & Estimate & $\delta$ & Estimate & $\delta$ \\
\hline 1 & 0.028 & -1.553 & 937.729 & 108.388 & -381.130 & 75.465 \\
2 & 0.035 & -1.456 & 951.567 & 52.525 & -292.228 & 26.100 \\
3 & 0.045 & -1.347 & 911.664 & 116.068 & -300.201 & 51.983 \\
4 & 0.056 & -1.252 & 831.603 & 50.314 & -266.360 & 19.626 \\
5 & 0.071 & -1.149 & 922.761 & 41.757 & -301.119 & 36.201 \\
6 & 0.089 & -1.051 & 821.773 & 45.834 & -320.040 & 30.526 \\
7 & 0.112 & -0.951 & 847.494 & 29.680 & -273.014 & 34.490 \\
8 & 0.141 & -0.851 & 805.118 & 24.375 & -323.978 & 34.030 \\
9 & 0.178 & -0.750 & 703.426 & 28.565 & -325.382 & 28.346 \\
10 & 0.224 & -0.650 & 755.704 & 34.303 & -271.443 & 33.988 \\
11 & 0.282 & -0.550 & 638.807 & 32.588 & -288.427 & 22.883 \\
12 & 0.355 & -0.450 & 619.953 & 27.855 & -266.322 & 32.370 \\
13 & 0.447 & -0.350 & 629.384 & 28.738 & -277.345 & 42.709 \\
14 & 0.562 & -0.250 & 658.969 & 39.606 & -295.290 & 43.924 \\
15 & 0.708 & -0.150 & 510.480 & 44.666 & -442.843 & 44.131 \\
16 & 0.891 & -0.050 & 486.201 & 68.351 & -474.615 & 75.602 \\
\hline
\end{tabular}

Table A.2. The Average $P_{1}^{0}$ Response in terms of Apparent Resistivity and Phase of Impedance. The error $\delta$ was derived from the jackknife standard errors on $Q$.

\begin{tabular}{|c|c|c|c|c|c|c|}
\hline \multirow{2}{*}{$\begin{array}{l}\text { Frequency } \\
\text { Band }\end{array}$} & \multicolumn{2}{|c|}{ Band Midpoint } & \multicolumn{2}{|c|}{$\left|\rho_{a}\right|$} & \multicolumn{2}{|l|}{$\phi$} \\
\hline & cpd & $\log (\mathrm{cpd})$ & Estimate & $\delta$ & Estimate & $\delta$ \\
\hline 1 & 0.028 & -1.553 & 2.620 & 0.890 & 67.880 & 19.800 \\
\hline 2 & 0.035 & -1.456 & 3.170 & 0.440 & 72.930 & 7.910 \\
\hline 3 & 0.045 & -1.347 & 3.790 & 1.210 & 71.770 & 18.690 \\
\hline 4 & 0.056 & -1.252 & 3.900 & 0.540 & 72.240 & 8.020 \\
\hline 5 & 0.071 & -1.149 & 6.110 & 0.870 & 71.930 & 8.150 \\
\hline 6 & 0.089 & -1.051 & 6.330 & 0.990 & 68.720 & 8.970 \\
\hline 7 & 0.112 & -0.951 & 8.110 & 1.020 & 72.140 & 7.240 \\
\hline 8 & 0.141 & -0.851 & 9.700 & 1.160 & 68.080 & 6.880 \\
\hline 9 & 0.178 & -0.750 & 9.770 & 1.300 & 65.180 & 7.660 \\
\hline 10 & 0.224 & -0.650 & 13.200 & 2.000 & 70.240 & 8.730 \\
\hline 11 & 0.282 & -0.550 & 12.660 & 1.830 & 65.700 & 8.330 \\
\hline 12 & 0.355 & -0.450 & 14.770 & 2.390 & 66.750 & 9.320 \\
\hline 13 & 0.447 & -0.350 & 19.320 & 3.660 & 66.220 & 10.930 \\
\hline 14 & 0.562 & -0.250 & 26.780 & 5.670 & 65.860 & 12.220 \\
\hline 15 & 0.708 & -0.150 & 29.550 & 7.170 & 49.060 & 14.040 \\
\hline 16 & 0.891 & -0.050 & 37.590 & 14.710 & 45.690 & 23.030 \\
\hline
\end{tabular}


Figure A.10. $H^{+}$Model: $d^{2}=10 \mathrm{ks}, \chi^{2}=8.75$.

A

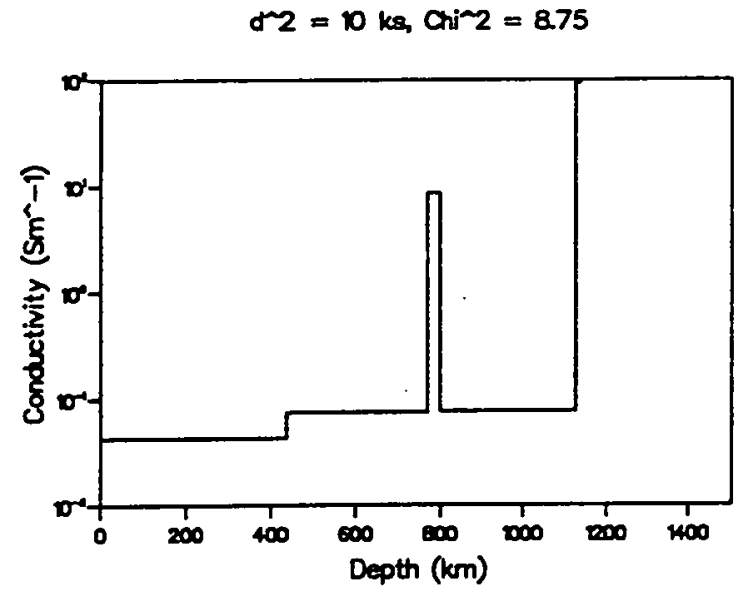

$\sigma^{\prime 2}=10 \mathrm{ks}, C h i^{-2}=8.75$

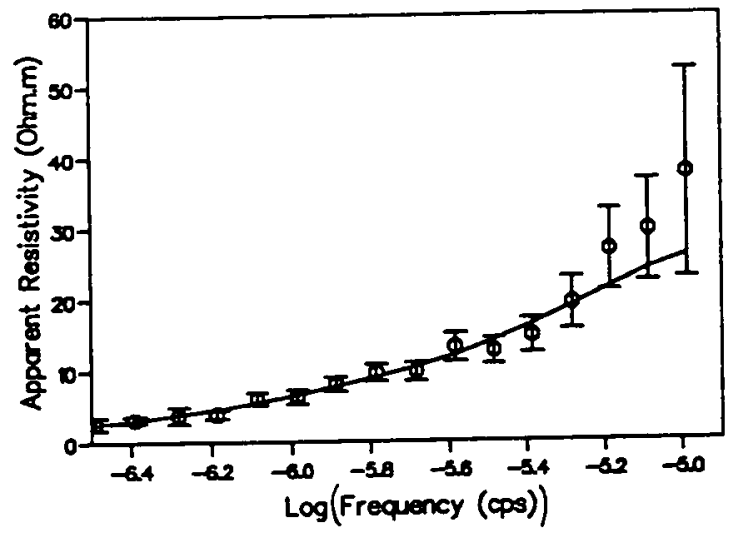

$\sigma_{2}=10 \mathrm{ks}, \mathrm{Chi}^{2}=8.75$

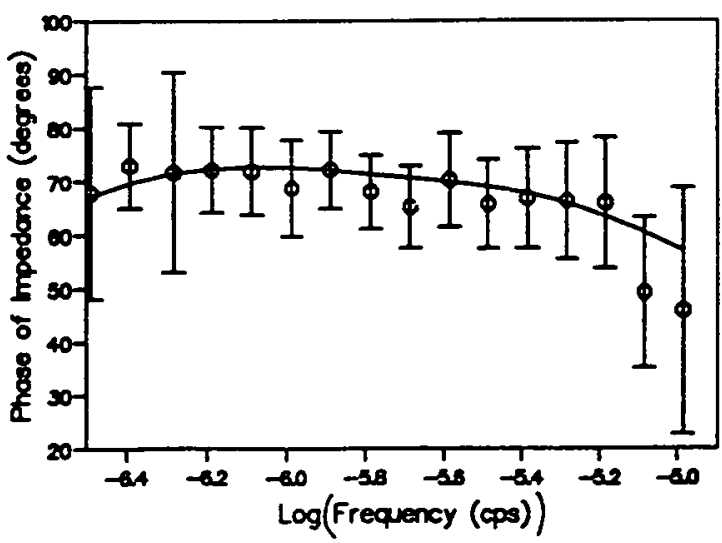


Figure A.11. $H^{+}$Model: $d^{2}=17.2 \mathrm{ks}, \chi^{2}=9.03$

B
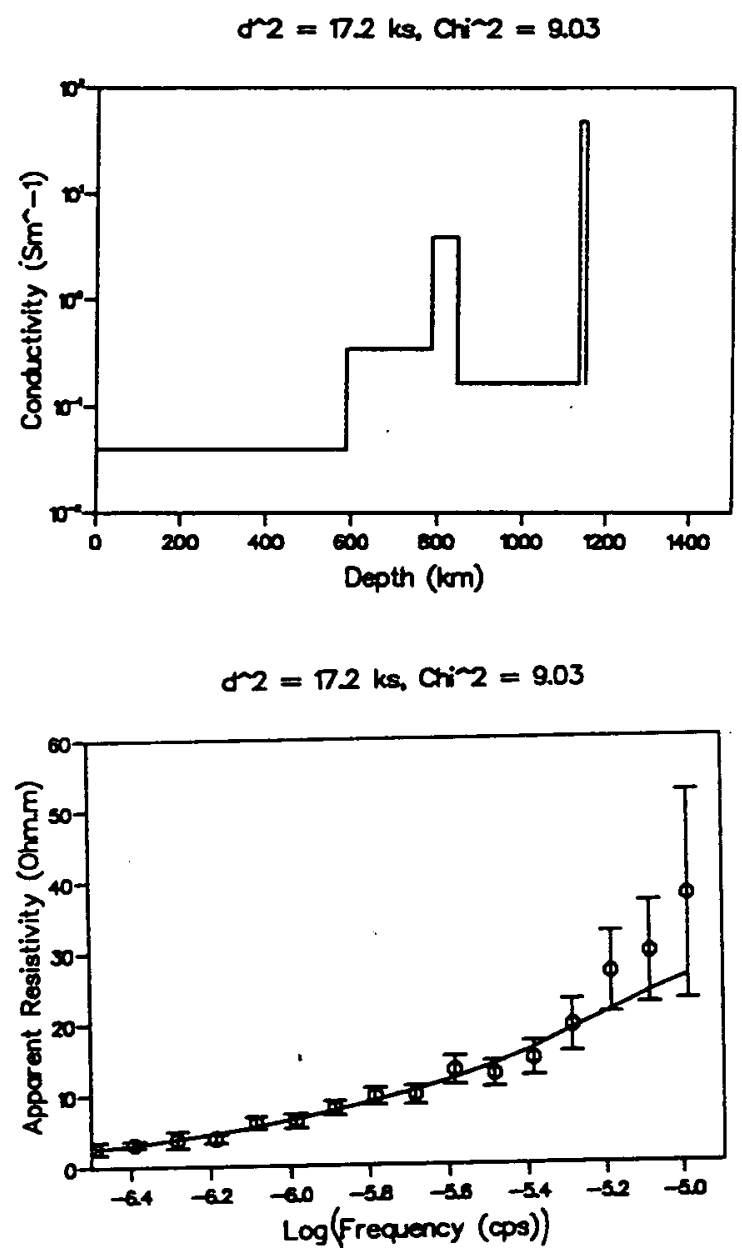

$\mathrm{dr}^{2}=172 \mathrm{ks}$, chir2 $=9.03$

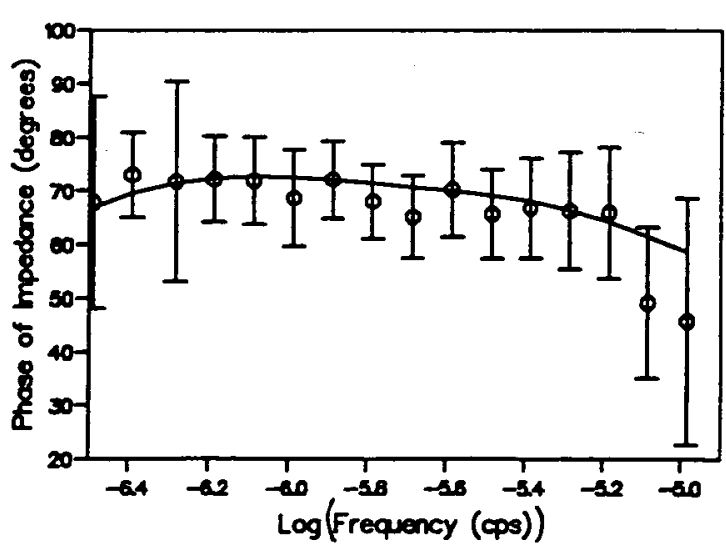


Figure A.12. $H^{+}$Model: $d^{2}=24.8 \mathrm{ks}, \chi^{2}=8.75$.

\section{C}

$ه 2=24.8 \mathrm{ks}$, Chi $2=8.75$

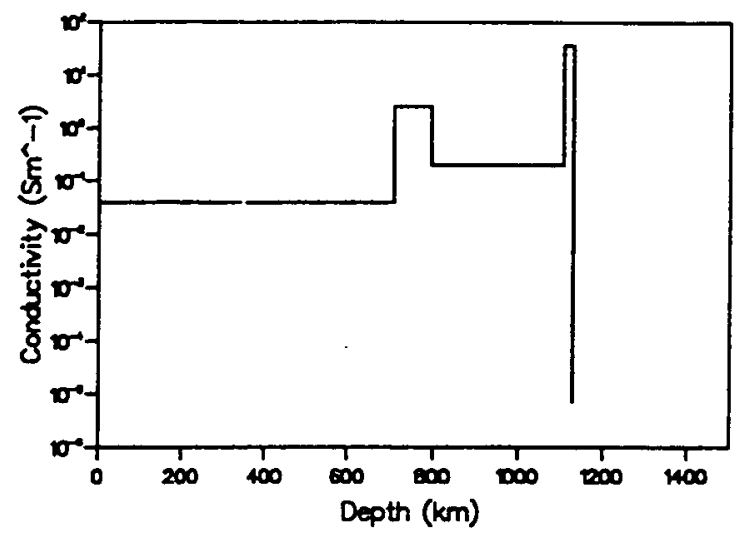

$\sigma_{2}=24.8 \mathrm{ks}, \mathrm{Chi}_{2}=8.75$

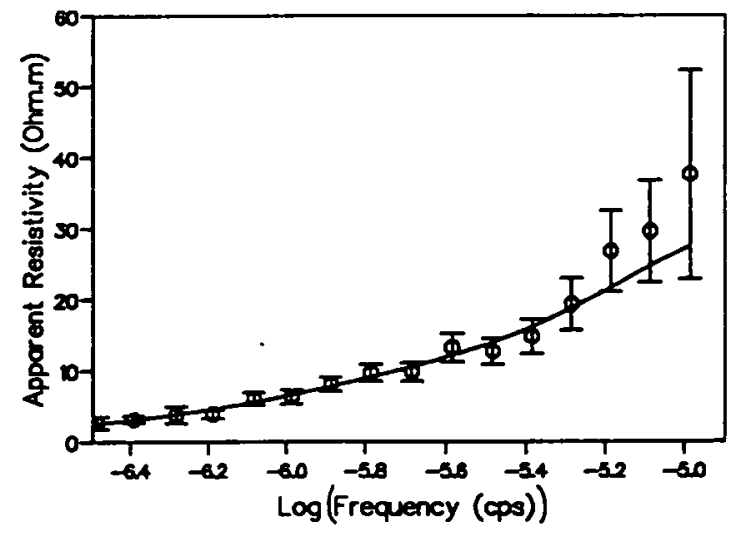

$\sigma^{\prime 2}=248 \mathrm{ks}, \mathrm{Chi}^{2} 2=8.75$

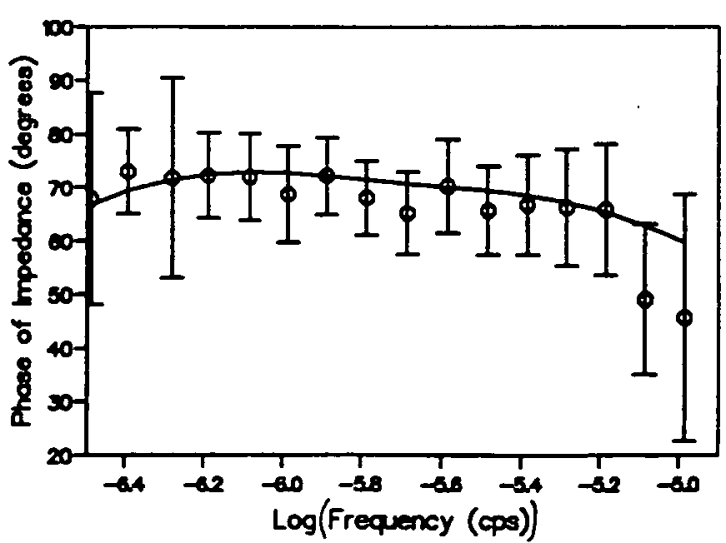


Figure A.13. $H^{+}$Model: $d^{2}=29$ ks, $\chi^{2}=8.58$.

D

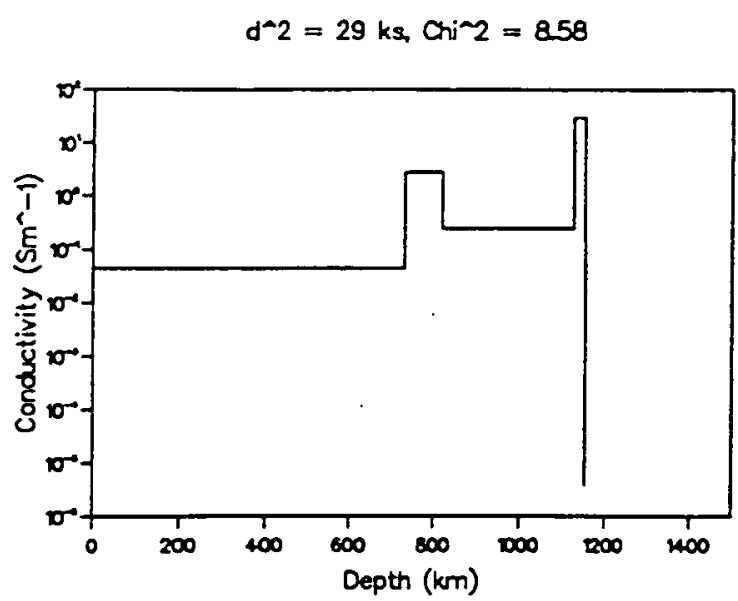

$\sigma 2=29 \mathrm{ks}, \sigma o r i^{2}=8.58$

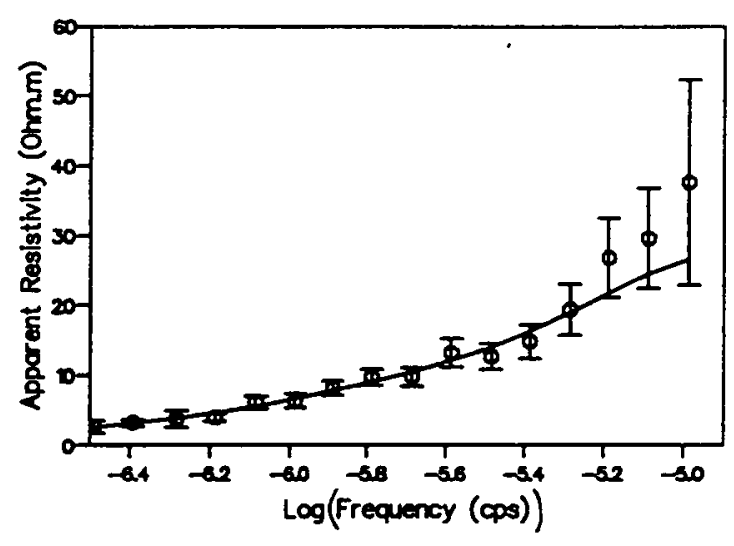

$\sigma_{2}=29 \mathrm{ks}, \mathrm{Chi}^{-2}=8.58$

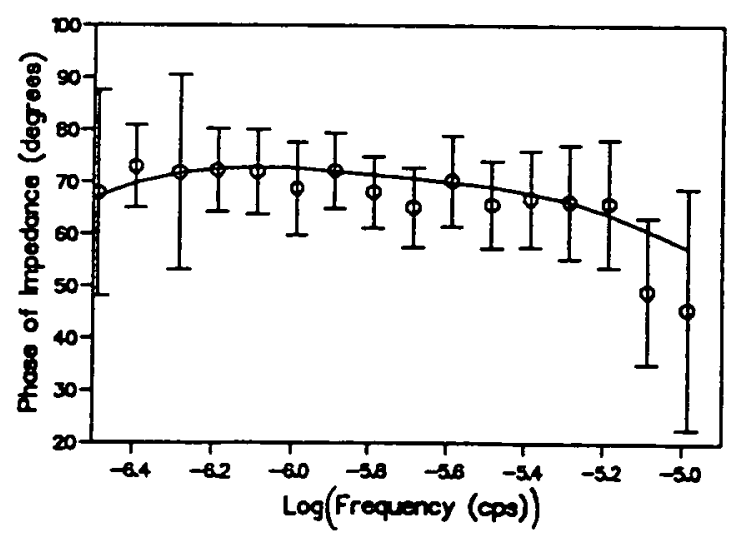


Figure A.14. $H^{+}$Model: $d^{2}=33.3 \mathrm{ks}, \chi^{2}=8.75$.

E

$d^{2}=33.3 \mathrm{~kg}, C O \mathrm{i}^{2} 2=8.75$

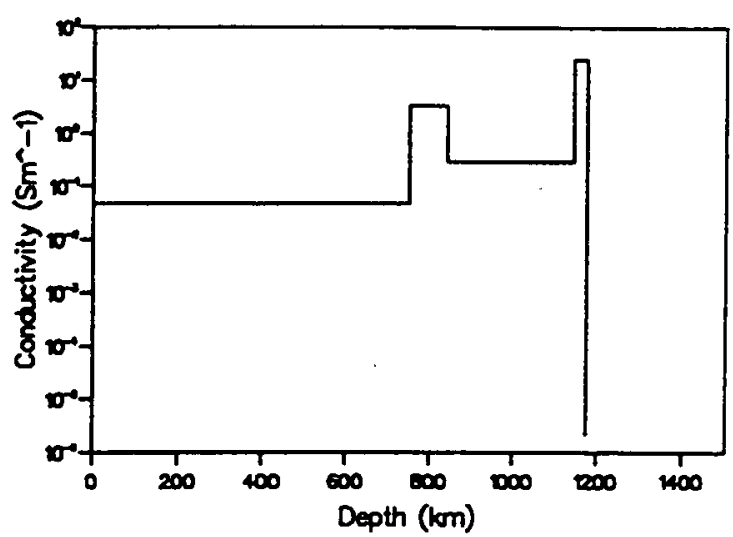

$\alpha_{2}=333 \mathrm{ks}, \mathrm{Cri}^{2} 2=8.75$

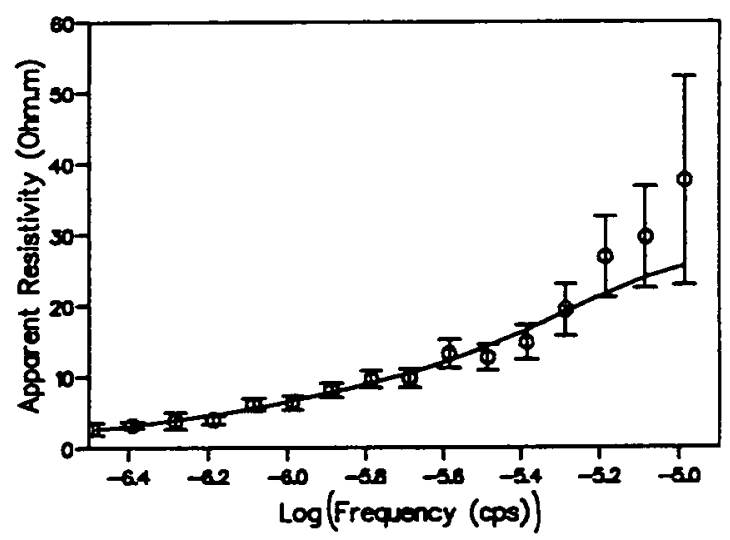

$\sigma 2=33.3 \mathrm{ks}, \mathrm{Cri}_{2}=8.75$

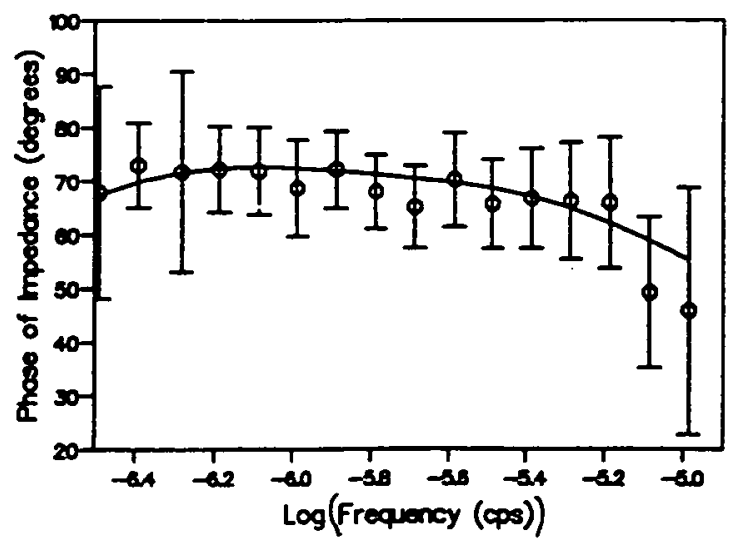

